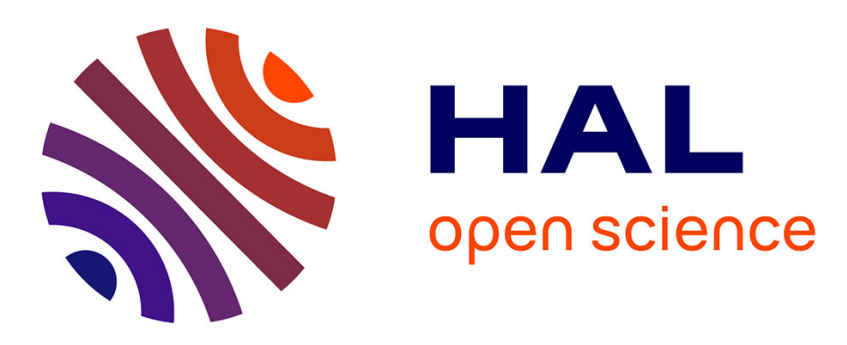

\title{
Mechanism and dynamics of fatty acid photodecarboxylase
}

Damien Sorigué, Kyprianos Hadjidemetriou, S. Blangy, G. Gotthard, A. Bonvalet, Nicolas Coquelle, P. Samire, Alexey Aleksandrov, L. Antonucci, A. Benachir, et al.

\section{To cite this version:}

Damien Sorigué, Kyprianos Hadjidemetriou, S. Blangy, G. Gotthard, A. Bonvalet, et al.. Mechanism and dynamics of fatty acid photodecarboxylase. Science, 2021, 372 (6538), pp.eabd5687. 10.1126/science.abd5687 . hal-03208662

\section{HAL Id: hal-03208662 https://hal.science/hal-03208662}

Submitted on 3 Sep 2021

HAL is a multi-disciplinary open access archive for the deposit and dissemination of scientific research documents, whether they are published or not. The documents may come from teaching and research institutions in France or abroad, or from public or private research centers.
L'archive ouverte pluridisciplinaire HAL, est destinée au dépôt et à la diffusion de documents scientifiques de niveau recherche, publiés ou non, émanant des établissements d'enseignement et de recherche français ou étrangers, des laboratoires publics ou privés. 


\section{Print page summary}

\section{Mechanism and dynamics of fatty acid photodecarboxylase}

Authors: D. Sorigué, K. Hadjidemetriou, S. Blangy, G. Gotthard, A. Bonvalet, N. Coquelle, P. Samire, A. Aleksandrov, L. Antonucci, A. Benachir, S. Boutet, M. Byrdin, M. Cammarata, S. Carbajo, S. Cuiné, R. B. Doak, L. Foucar, A. Gorel, M. Grünbein, E. Hartmann, R. Hienerwadel, M. Hilpert, M. Kloos, T. J. Lane, B. Légeret, P. Legrand, Y. Li-Beisson, S. L. Y. Moulin, D. Nurizzo, G. Peltier, G. Schirò, R. L. Shoeman, M. Sliwa, X. Solinas, B. Zhuang, T. R.M. Barends, J.-P. Colletier, M. Joffre, A. Royant, C. Berthomieu*, M. Weik*, T. Domratcheva*, K. Brettel, M. H. Vos*, I. Schlichting*, P. Arnoux*, P. Müller*, F. Beisson*

Introduction: Photoenzymes are rare biocatalysts driven by absorption of a photon at each catalytic cycle; they inspire development of artificial photoenzymes with valuable activities. Fatty acid photodecarboxylase (FAP) is a natural photoenzyme that has potential applications in the biobased production of hydrocarbons, yet its mechanism is far from fully understood.

Rationale: To elucidate the mechanism of FAP, we studied the wild type enzyme from Chlorella variabilis $(C \nu \mathrm{FAP})$ and variants with altered active site residues using a wealth of techniques, including static and time-resolved crystallography and spectroscopy as well as biochemical and computational approaches.

Results: A $1.8 \AA$ A-resolution $C v$ FAP x-ray crystal structure revealed a dense hydrogen bonding network positioning the fatty acid carboxyl group in the vicinity of the flavin adenine dinucleotide (FAD) cofactor. Structures solved from free electron laser and low-dose synchrotron x-ray crystal data further highlighted an unusual bent shape of the oxidized flavin chromophore and show that 
the bending angle $\left(14^{\circ}\right)$ does not change upon photon absorption (step 1) and throughout the photocycle. Calculations show that bending substantially affects the energy levels of the flavin. Structural and spectroscopic analysis of wild-type (WT) and mutant proteins targeting two conserved active site residues, R451 and C432, demonstrated that both residues are crucial for proper positioning of the substrate and water molecules, and oxidation of the fatty acid carboxylate by ${ }^{1} \mathrm{FAD}^{*}$ ( 300 ps in WT FAP) to form $\mathrm{FAD}^{\bullet-}$ (step 2). Time-resolved infrared spectroscopy demonstrated that decarboxylation occurs quasi-instantaneously upon this forward electron transfer, consistent with barrierless bond cleavage predicted by quantum chemistry calculations and with snapshots obtained by time-resolved crystallography. Transient absorption spectroscopy in $\mathrm{H}_{2} \mathrm{O}$ and $\mathrm{D}_{2} \mathrm{O}$ buffers indicated that back electron transfer from $\mathrm{FAD}^{\bullet-}$ is coupled to and limited by transfer of an exchangeable proton or hydrogen atom (step 3). Unexpectedly, concomitant with $\mathrm{FAD}^{\bullet-}$ reoxidation (to a red-shifted form $\mathrm{FAD}_{\mathrm{RS}}$ ) in $100 \mathrm{~ns}$, the majority of the $\mathrm{CO}_{2}$ product is converted, most likely into bicarbonate (as inferred from FTIR spectra of the cryo-trapped FADRS intermediate). Calculations indicate that this catalytic transformation involves an active-site water molecule. Cryo-Fourier transform infrared spectroscopy studies suggested that bicarbonate formation (step 4) is preceded by deprotonation of an arginine residue (step 3). At room temperature, the remaining $\mathrm{CO}_{2}$ leaves the protein in $1.5 \mu$ s (step 4'). The observation of residual electron density close to C432 in electron density maps derived from time-resolved and cryocrystallography data suggests that this residue may play a role in stabilizing $\mathrm{CO}_{2}$ and/or bicarbonate. Three routes for alkane formation were identified by quantum chemistry calculations; the one shown in the figure is favored by the ensemble of experimental data.

Conclusion: We provide a detailed and comprehensive characterization of light-driven hydrocarbon formation by fatty acid photodecarboxylase, which uses a remarkably complex 
mechanism including unique catalytic steps. We anticipate that our results will help expand the green chemistry toolkit.

\section{Figure Caption:}

Elucidation of the FAP photocycle by combining spectroscopic, biochemical, crystallographic and computational studies.

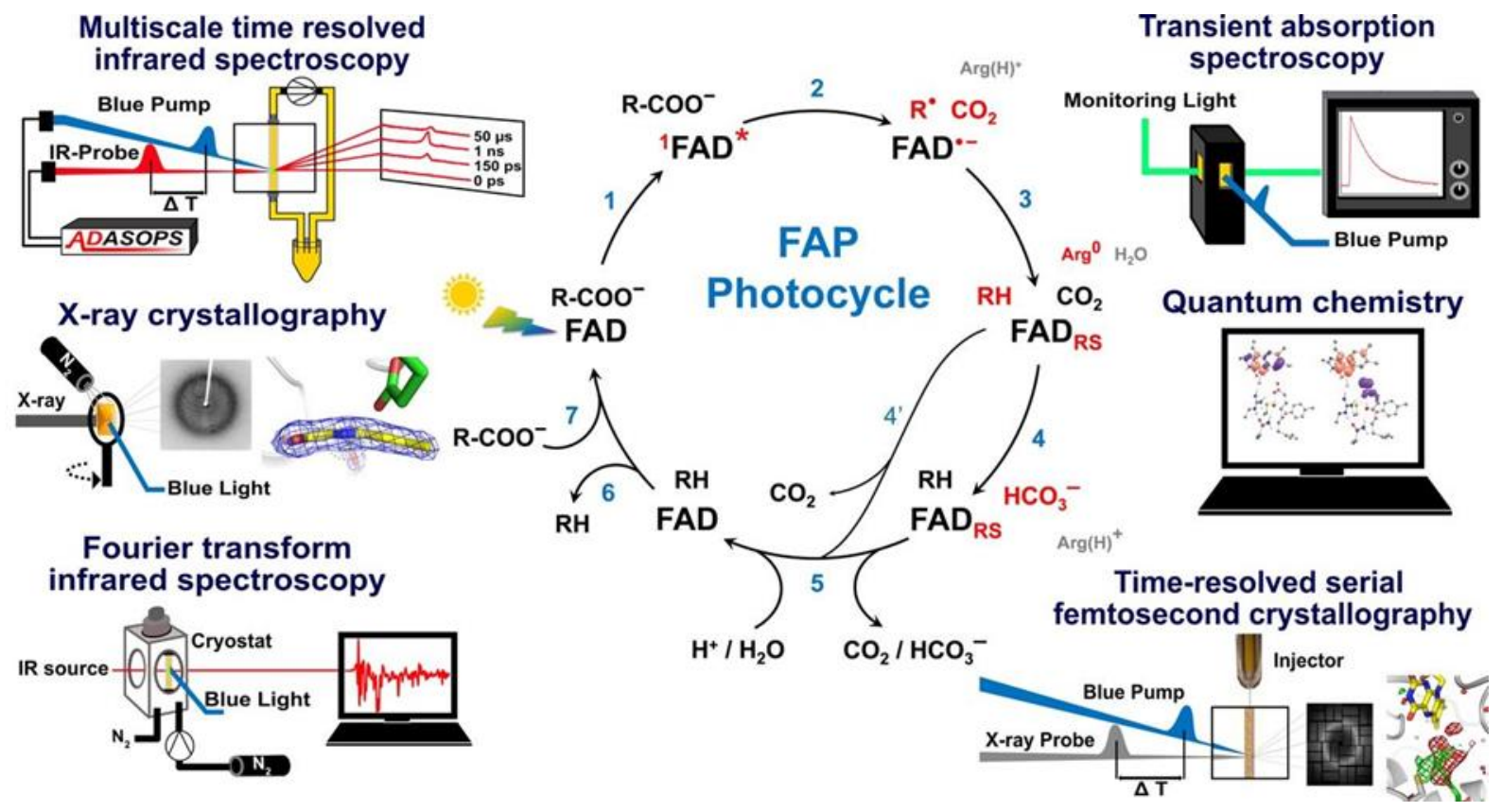

The list of author affiliations is available in the full article online. *Corresponding author. Email: frederic.beisson@cea.fr (F.B.); pavel.muller@i2bc.paris-saclay.fr (P.M.); pascal.arnoux@cea.fr (P.A.); Ilme.Schlichting@mpimf-heidelberg.mpg.de (I.S.); marten.vos@polytechnique.edu (M.H.V.); Tatjana.Domratcheva@mpimf-heidelberg.mpg.de (T.D.); martin.weik@ibs.fr (M.W.); catherine.berthomieu@cea.fr (C.B.); 
2 Authors: D. Sorigué ${ }^{1}$, K. Hadjidemetriou ${ }^{2}$, S. Blangy ${ }^{1}$, G. Gotthard ${ }^{3}$, A. Bonvalet ${ }^{4}$, N. Coquelle ${ }^{5}$,

3 P. Samire ${ }^{1,6}$, A. Aleksandrov ${ }^{4}$, L. Antonucci ${ }^{4}$, A. Benachir ${ }^{4}$, S. Boutet ${ }^{7}$, M. Byrdin ${ }^{2}$, M.

4 Cammarata $^{8}$, S. Carbajo ${ }^{7}$, S. Cuiné ${ }^{1}$, R. B. Doak ${ }^{9}$, L. Foucar ${ }^{9}$, A. Gorel ${ }^{9}$, M. Grünbein ${ }^{9}$, E.

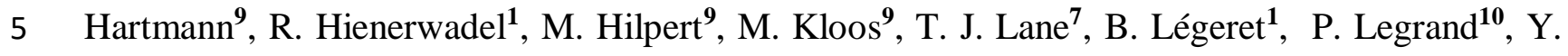

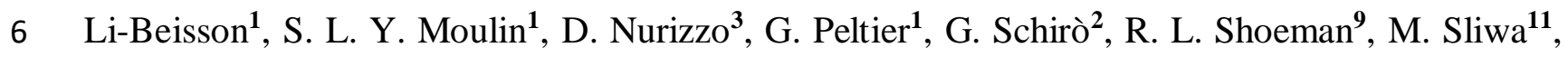

7 X. Solinas', B. Zhuang ${ }^{4,6}$, T. R. M. Barends 9 , J.-P. Colletier ${ }^{2}$, M. Joffre ${ }^{4}$, A. Royant ${ }^{2,3}$, C.

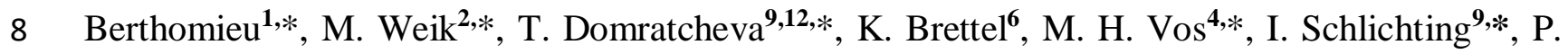

$9 \operatorname{Arnoux}^{1, *}$, P. Müller ${ }^{6}, *$, F. Beisson ${ }^{1, *}$

10 Affiliations:

$11{ }^{1}$ Aix-Marseille University, CEA, CNRS, Institute of Biosciences and Biotechnologies, BIAM

12 Cadarache, 13108 Saint-Paul-lez-Durance, France.

$13{ }^{2}$ Univ. Grenoble Alpes, CEA, CNRS, Institut de Biologie Structurale, 38000 Grenoble, France.

$14{ }^{3}$ European Synchrotron Radiation Facility, 71 Avenue des Martyrs, CS 40220, 38043 Grenoble, 15 France.

$16{ }^{4}$ LOB, CNRS, INSERM, Ecole Polytechnique, Institut Polytechnique de Paris, 91128, Palaiseau, 17 France.

$18{ }^{5}$ Large-Scale Structures Group, Institut Laue Langevin, 71, avenue des Martyrs, 38042 Grenoble 19 cedex 9, France.

$20{ }^{6}$ Université Paris-Saclay, CEA, CNRS, Institute for Integrative Biology of the Cell (I2BC), 91198 21 Gif-sur-Yvette, France. 
$1 \quad{ }^{7}$ Linac Coherent Light Source (LCLS), SLAC National Accelerator Laboratory, 2575 Sand Hill

2 Road, Menlo Park, CA 94025, USA.

$3 \quad{ }^{8}$ Department of Physics, UMR UR1-CNRS 6251, University of Rennes 1, F- Rennes, France:

4 Marco Cammarata (Current address: ESRF)

$5 \quad{ }^{9}$ Max-Planck-Institut für medizinische Forschung, Jahnstrasse 29, 69120 Heidelberg, Germany.

$6{ }^{10}$ Synchrotron SOLEIL. L'Orme des Merisiers Saint-Aubin, BP 48, 91192 Gif-sur-Yvette, France.

7 11 Univ. Lille, CNRS, UMR 8516, LASIR, Laboratoire de Spectrochimie Infrarouge et Raman,

859000 Lille, France.

$9 \quad{ }^{12}$ Department of Chemistry, Lomonosov Moscow State University, Moscow 119991, Russia.

$10 *$ Correspondence to: frederic.beisson@cea.fr (F.B.), pavel.muller@i2bc.paris-saclay.fr (P.M.),

11 pascal.arnoux@cea.fr (P.A.), Ilme.Schlichting@mpimf-heidelberg.mpg.de (I.S.),

12 marten.vos@polytechnique.edu (M.H.V.), Tatjana.Domratcheva@mpimf-heidelberg.mpg.de

13 (T.D.), martin.weik@ibs.fr (M.W.), catherine.berthomieu@cea.fr (C.B.) 
1 Abstract: Fatty Acid Photodecarboxylase (FAP) is a photoenzyme with potential green chemistry

2 applications. By combining static, time-resolved, and cryotrapping spectroscopy and

3 crystallography as well as computation, we characterize Chlorella variabilis FAP reaction

4 intermediates on time scales from sub-picoseconds to milliseconds. High-resolution crystal

5 structures from synchrotron and free electron laser x-ray sources highlight an unusual bent shape

6 of the oxidized flavin chromophore. We demonstrate that decarboxylation occurs directly upon

7 reduction of the excited flavin by the fatty acid substrate. Along with flavin reoxidation by the alkyl

8 radical intermediate, a major fraction of the cleaved $\mathrm{CO}_{2}$ unexpectedly transforms in $100 \mathrm{~ns}$, most

9 likely into bicarbonate. This reaction is orders of magnitude faster than in solution. Two strictly

10 conserved residues, R451 and C432, are essential for substrate stabilization and functional charge

11 transfer.

14 One Sentence Summary: Snapshots of a photoenzyme reveal reaction intermediates during

15 fatty acid decarboxylation.

16

17 
1 The vast majority of enzymatic reactions in living cells are thermally activated, whereas reactions

2 driven by light are much less common (1). Apart from photosynthetic reaction centers, three natural

3 photoenzymes have been identified to date: DNA photolyases, which are involved in the repair of

4 UV-damaged DNA (2), light-dependent protochlorophyllide oxidoreductase (LPOR) required for

5 the maturation of chlorophyll (3) and the recently discovered fatty acid photodecarboxylase (FAP,

6 EC 4.1.1.106), which converts fatty acids to hydrocarbons and $\mathrm{CO}_{2}(4)$.

Photoenzymes require a photon for each turnover of a substrate molecule and offer the

8 possibility to trigger and monitor catalytic steps and associated structural changes on very short

9 time scales that are generally not accessible for thermally-activated enzymes (5). Thus, over the

10 past 20 years, the photochemical mechanism has been studied in detail for DNA photolyases (6-

11 8) and has started to be identified for LPOR (9-13). Due to its readily available substrate, FAP may

12 become a model of choice to understand catalytic steps that occur in enzymology.

Light-driven enzymes are also interesting for practical applications. Increasing our

14 understanding and the repertoire of photoenzymatic mechanisms may help in the design of catalysts

15 performing new reactions $(14,15)$ or in the development of new light-controlled proteins for optogenetics (16). FAP has potential biotechnological applications in green chemistry because

17 hydrocarbons are important as cosmetics emollients, chemical synthons, solvents and fuels (17-

18 19). FAP complements routes previously identified for bio-based synthesis of hydrocarbons (20)

19 by providing a one-step light-driven pathway from fatty acids. Rational design approaches have

20 recently allowed to improve the efficiency of FAP on high-value functionalized carboxylic acids

21 (21) and on short-chain fatty acids in order to produce liquefied petroleum gas (20). Understanding

22 the reaction mechanism of FAP in detail is thus of utmost importance, both from a fundamental-

23 research and an application point of view. 
FAP is an algae-specific enzyme from the glucose-methanol-choline (GMC)

2 oxidoreductase family harboring a flavin adenine dinucleotide (FAD) cofactor. It allows the

3 decarboxylation of C16-C18 free (non-esterified) fatty acids to the corresponding $n$-alka(e)nes (4).

$4 \quad$ These hydrocarbon products are mostly located in chloroplast thylakoids but their exact role is still

5 unknown $(22,23)$. The initial spectroscopic characterization of FAP, based on monitoring the

6 electronic state of the flavin following excitation by a laser flash, has led to a first model of the

7 FAP photocycle (4). The cycle starts with the quenching of the singlet excited state by forward

8 electron transfer (fET) from bound fatty acid $\mathrm{R}-\mathrm{CO}_{2}{ }^{-}$in $\sim 300$ ps (with a quantum yield $>80 \%$ ),

9 forming a flavin anion radical $\mathrm{FAD}^{\bullet-}$ and a fatty acid radical $\mathrm{R}-\mathrm{CO}_{2} \bullet$. The latter decarboxylates,

10 yielding an alkyl radical $\mathrm{R}^{\bullet}$ and $\mathrm{CO}_{2} . \mathrm{FAD}^{\bullet-}$ is re-oxidized in $\sim 100$ ns by back electron transfer

11 (bET) which ultimately provides the electron for the reduction of $\mathrm{R}^{\bullet}$ to the alkane $\mathrm{RH}^{\mathrm{F}} \mathrm{FAD}^{\bullet-}$

12 reoxidation results in a transiently red-shifted flavin state $\mathrm{FAD}_{\mathrm{RS}}$ that reverts to the initial state in

$134 \mathrm{~ms}$.

Despite this insight, several open questions remained, such as: which structural features of

15 the FAP active site promote substrate stabilization and favor the fET? Is decarboxylation

16 instantaneous upon this fET step or is it slowed by an activation barrier? Does conversion of $\mathrm{R}^{\circ}$ to

17 the alkane $\mathrm{RH}$ occur by bET from $\mathrm{FAD}^{\bullet-}$ coupled to a proton transfer (PT/PCET) or by hydrogen

18 atom transfer (HAT) from a nearby amino acid $(4,24)$ ? What is the origin of the proton or the

19 hydrogen atom? Here, we report a high-resolution structure of FAP and characterize key steps

20 along the FAP photocycle using a wealth of static and time-resolved crystallographic and

21 spectroscopic techniques, as well as computational approaches. Our detailed characterization of

22 FAP reveals unforeseen mechanistic complexity. 


\section{High resolution structure of $C v \mathbf{F A P}$}

2 Crystals of FAP from Chlorella variabilis NC64A ( $C v \mathrm{FAP})$, diffracting x-rays to high resolution

3 and without the twinning fault reported earlier (4), were obtained upon removal of the N-terminal

4 helix involved in crystal packing (residues 61 to 76 ) in native $C v \mathrm{FAP}$. The resulting structure,

5 solved at $1.8 \AA$ resolution (table S1) now provides a detailed view of the active site architecture

6 (Fig. 1A-D and fig. S1). Although no substrate was added during protein purification and

7 crystallization, two C18 fatty acids co-purified with FAP were clearly identified, one in the active

8 site, the other on the surface of the protein. The latter is stabilized by hydrophobic interactions with

9 nonpolar side chains (L427, Y419, I126, I416 and L420) as well as the aliphatic parts of R132 and

10 R122. In the active site, the carboxyl group of the fatty acid is stabilized by hydrogen bonds with

11 water molecules (Wat1 and Wat2) and the side chains of R451 and N575 (Fig. 1D). The fatty acid

12 substrate is also stabilized on the dimethylbenzene side of FAD, in contrast to other GMC 13 oxidoreductase enzymes that have their substrate stabilized near the N5 atom of FAD (fig. S2).

\section{Conformation of oxidized FAD in CvFAP}

16 In the high-resolution dark-state structure of FAP obtained from synchrotron data ' $100 \mathrm{~K}$ dark',

17 the isoalloxazine ring of the FAD cofactor was found to be bent, with the dihedral angle C4-N5-

18 N10-C9 (butterfly bending angle) deviating by $17.4^{\circ}$ from planarity (Fig. 1E, fig S2A). Such

19 bending is usually interpreted as caused by X-ray photoreduction converting supposedly planar

20 oxidized flavin to the bent reduced form $(8,25,26)$. For FAP however, in crystallo UV-Vis

21 absorption microspectrophotometry (fig. S3), low dose crystallography, (fig. S4A and table S1),

22 Raman microspectrophotometry (fig. S4B,C) and molecular dynamics simulations (fig. S5A,B) 
1 using a recent flavin force field (27) indicated that the FAD cofactor is bent in its oxidized form

2 (supplementary text S1). To obtain a definite answer concerning the conformation of the oxidized

3 FAD in FAP, we performed room temperature serial femtosecond crystallography (SFX) at an X-

4 ray free-electron laser (XFEL; see below for details), which allows collecting essentially radiation

5 damage-free diffraction data $(28,29)$. Importantly, this SFX dark-state structure of FAP ('SFX

6 dark'), solved at $2.0 \AA$ A resolution (table S2) features a FAD with a similar bending angle $\left(14.3^{\circ}\right)$ as

7 observed in the synchrotron ' $100 \mathrm{~K}$ dark' and the 'RT dark low-dose' structures (Fig. 1E and figs.

$8 \mathrm{~S} 4, \mathrm{~S} 6, \mathrm{~S} 7)$, supporting the notion that in FAP, the FAD cofactor is in a bent conformation in the

9 oxidized state. To our knowledge, a butterfly bent conformation of the oxidized flavin has not been

10 firmly established for any other flavoprotein. In fact, bent conformations were either not discussed

11 or attributed to X-ray-induced flavin reduction. Future radiation-damage-free structures of oxidized

12 flavoproteins should reveal whether the bending is a feature specific to FAP.

\section{New insights into $C v$ FAP photocycle by time-resolved spectroscopy}

15 Our previous single-shot fluorescence and transient absorption experiments in the presence of 16 substrate, with 100 ps and 10 ns temporal resolution, respectively, showed a decay of the singlet 17 excited flavin $\left({ }^{1} \mathrm{FAD}^{*}\right)$ in $\sim 300 \mathrm{ps}$ and formation of $\mathrm{FAD}^{\bullet-}$ within $10 \mathrm{~ns}(4)$. Here we extend the

18 fluorescence and visible absorption experiments to the ultrafast time scale with 100 fs-resolution 19 pump-probe spectroscopy (Fig. 2A,B and fig. S8A) showing that no faster ${ }^{1}$ FAD* decay phases 20 occur, (only a $\sim 2$ ps thermal relaxation phase was observed in the fluorescence as in other 21 flavoproteins (30)) and that, as predicted (4), $\mathrm{FAD}^{\bullet-}$ is formed concomitant with ${ }^{1} \mathrm{FAD} *$ decay (Fig $22 \mathrm{~S} 8 \mathrm{~B})$. 
We investigated whether the carboxylate of the fatty acid is cleaved off concomitantly with

2 electron transfer from the fatty acid to ${ }^{1} \mathrm{FAD}^{*}(\sim 300 \mathrm{ps})$, after this oxidation, or accompanying the

3 bET from flavin $(\sim 100 \mathrm{~ns})$. Light-induced FTIR difference spectroscopy performed at $298 \mathrm{~K}$

4 showed a $\mathrm{CO}_{2}$ band at $2342 \mathrm{~cm}^{-1}$ (Fig. 2C) corresponding to $\mathrm{CO}_{2}$ in solution (31). The kinetics of

$5 \mathrm{CO}_{2}$ formation in FAP were monitored by picosecond to microsecond time-resolved infrared

6 spectroscopy (TR-IR) in a frequency range centered around $2350 \mathrm{~cm}^{-1}$ and Arbitrary Detuning

7 ASynchronous OPtical Sampling (ADASOPS) $(32,33)$. This experiment showed the appearance

8 of a $\mathrm{CO}_{2}$ absorption band centered at $2337.5 \mathrm{~cm}^{-1}$ with a time constant of $\sim 270$ ps (Fig. 2C-E and

9 fig. S9). We conclude that decarboxylation is rate-limited by electron transfer from substrate to

$10{ }^{1} \mathrm{FAD} *$ occurring in $\sim 300 \mathrm{ps}$. The initial $\mathrm{CO}_{2}$ band frequency is $\sim 5 \mathrm{~cm}^{-1}$ down-shifted with respect

11 to that of ${ }^{12} \mathrm{CO}_{2}$ in aqueous solution, a finding we assign to the protein environment. Subsequently,

12 the $\mathrm{CO}_{2}$ signal diminishes 4-fold with a time constant of $100 \mathrm{~ns}$, without changing much in shape,

13 followed by an upshift towards $2342 \mathrm{~cm}^{-1}$, a process fitted with a time constant of $\sim 1.5 \mu$ s. The

14 latter process likely reflects migration of $\mathrm{CO}_{2}$ towards the solvent. Importantly, the data indicate

15 that the $100 \mathrm{~ns}$ process implies transformation of $\sim 75 \%$ of the initially formed $\mathrm{CO}_{2}$ within the

16 protein into another molecule, possibly bicarbonate, concomitant with flavin reoxidation to the red-

17 shifted form $\mathrm{FAD}_{\mathrm{RS}}$ (see section on red-shift intermediate). This process was not foreseen in our

18 previously proposed reaction scheme (4). Whereas our present data covering six orders of

19 magnitude in time can reasonably well be described with these three exponential processes and a

20 constant phase (Fig. 2E), it is possible that an additional phase of $\mathrm{CO}_{2}$ release into the solvent

21 occurs on a timescale exceeding $50 \mu \mathrm{s}$, the temporal window of our present experiments.

In order to determine whether any of the reaction steps are coupled to or directly reflect a

23 proton transfer (PT) or a hydrogen atom transfer (HAT), we compared the kinetics of all steps 
1 observable by continuous-probe time-resolved fluorescence or transient absorption spectroscopy

2 in $\mathrm{H}_{2} \mathrm{O}$ and $\mathrm{D}_{2} \mathrm{O}$ buffers. The biphasic fluorescence signal showing the decay of ${ }^{1} \mathrm{FAD} *$ (due to

3 quenching by ET from the substrate (300 ps phase; > 80\%) and to the intersystem-crossing to a non-

4 reactive triplet state $(\sim 6.5 \mathrm{~ns}$ phase; $<20 \%))$ was not visibly affected by the isotope exchange (Fig.

5 3A), as expected for reactions that are not significantly coupled to movements of exchangeable

6 hydrogen species (e.g., back-ET from $\mathrm{Q}_{\mathrm{A}}^{-}$to $\mathrm{P} 680^{+}$in photosystem II (34)). In contrast, the kinetics

7 of $\mathrm{FAD}^{\bullet-}$ reoxidation to $\mathrm{FAD}_{\mathrm{RS}}$ observed by transient absorption spectroscopy at three

8 characteristic wavelengths slowed down by a factor of $\sim 2$ : from $\sim 100 \mathrm{~ns}$ in $\mathrm{H}_{2} \mathrm{O}$ to $\sim 200 \mathrm{~ns}$ in $\mathrm{D}_{2} \mathrm{O}$

9 (Fig. 3B), suggesting that this bET step is coupled to and limited by transfer of an exchangeable proton or hydrogen atom. Change of buffer $\mathrm{pH}$ in the interval between 7.5 and 9.1 had no effect on

11 the kinetics of this step (fig. S10A), indicating that the proton or hydrogen atom donor involved in

12 the reaction has a $\mathrm{p} K_{\mathrm{a}}$ value above 9.1. The significantly lower kinetic isotope effect (KIE) of 1.2

13 reported by Heyes et al. (24) might be due to insufficient time-resolution of their experiment (see

14 supplementary text S4).

The last step observable by transient absorption spectroscopy is the disappearance of the transient red shift of reoxidized FAD occurring in a few milliseconds. This process was previously (4) assigned to reprotonation of $\mathrm{X}^{-}$, the conjugate base of $\mathrm{XH}$, an unidentified proton donor to the

18 alkyl radical. Kinetics of this process also slowed down when $\mathrm{H}_{2} \mathrm{O}$ in the buffer was replaced by

$\mathrm{D}_{2} \mathrm{O}$ : from $\sim 3 \mathrm{~ms}$ to $\sim 10 \mathrm{~ms}$ (Fig. 3C). Consistent with this observation and the recent report of a

$20 \mathrm{pH}$ increase (in an unbuffered solution) associated with this step (24), this process apparently

21 reflects a proton transfer from bulk solvent. Again, change of buffer $\mathrm{pH}$ in the interval between 7.5

22 and 9.1 had no effect on the kinetics of this step (fig. S10B) and neither did the consumption of the

23 native substrate (fig. S10C). Time-resolved spectroscopic findings are summarized in fig. S11. 
2 Photodecarboxylation and characterization of the red-shifted intermediate at cryogenic

3 temperatures

4 Our time-resolved spectroscopic results predict that a fraction of the $\mathrm{CO}_{2}$ product is present in the

5 active site in the red-shifted photoproduct intermediate. For a detailed characterization by static

6 methods, we tried to stabilize this intermediate by cryotrapping. UV-visible absorption spectra of

$7 \quad C \nu$ FAP crystals and solutions exposed to increasing amounts of blue light at $100 \mathrm{~K}$ indeed revealed

8 the gradual conversion into a red-shifted form similar to that previously observed at room

9 temperature (Ref. (4) and fig. S12). These conditions were used to further characterize the red-

10 shifted intermediate using light-induced FTIR difference spectroscopy. In order to identify IR

11 modes of the substrate and products of the reaction, we replaced the native substrate by $1-{ }^{12} \mathrm{C}$ or 1 -

$12{ }^{13} \mathrm{C}$ palmitate. The identification of characteristic ${ }^{13} \mathrm{C}$-sensitive IR bands of carboxylate in the FTIR

13 difference spectra at $1541 \mathrm{~cm}^{-1}$ and $1391 \mathrm{~cm}^{-1}$ shows that the substrate initially was in the

14 deprotonated form (Fig. 4A $i$ and $i i$; details supporting all IR bands assignments mentioned below

15 are given in supplementary text S2). A peak at $2340 \mathrm{~cm}^{-1}$ (Fig. 4 A panel $v$ ) was assigned to

16 formation of $\mathrm{CO}_{2}$ from $1{ }^{1-}$ Cpalmitate and at $2274 \mathrm{~cm}^{-1}$ from $1-{ }^{13} \mathrm{C}$-palmitate. When comparing

17 FTIR spectra recorded with FAP samples containing $1-{ }^{12} \mathrm{C}$ - and $1-{ }^{13} \mathrm{C}$-palmitate, small bands were

18 also observed at $1356-1335 \mathrm{~cm}^{-1}$ and $1312 \mathrm{~cm}^{-1}$ (Fig. 4A $\mathrm{iii}$ ), which is indicative of the formation

19 of trace amounts of ${ }^{12} \mathrm{C}$ and ${ }^{13} \mathrm{C}$ bicarbonate respectively. We repeated the FTIR experiments at

$20150 \mathrm{~K}$. At this temperature, the $\mathrm{CO}_{2}$ band was small (Fig. 4A panel $v$ ), while large positive bands

21 at 1646 (1614) and $1352(1318) \mathrm{cm}^{-1}$ were detected that can be unambiguously assigned to IR

22 modes of ${ }^{12} \mathrm{C}-\left({ }^{13} \mathrm{C}\right.$-) bicarbonate (Fig. 4A $\left.i v\right)$. The FTIR data thus demonstrate the build-up of

23 bicarbonate at $150 \mathrm{~K}$ and indicate that its formation at $100 \mathrm{~K}$ is limited by an energy barrier. 
2 light') featured FAP trapped in a $\mathrm{FAD}_{\mathrm{RS}}$ state, as shown by in crystallo microspectrophotometry

3 (fig. S12). The difference electron density map between ' $100 \mathrm{~K}$ light' and ' $100 \mathrm{~K}$ dark' displays

$4 \quad$ strong and significant peaks only around the substrate and FAD cofactor. These peaks can be fitted

5 with a $\mathrm{CO}_{2}$ molecule in addition to the alkyl chain stabilized in the active site (Fig. $\mathbf{4 B}, \mathbf{C}$ and fig.

6 S13A-C). The positive difference electron density along the aliphatic tail of the substrate (from

$7 \mathrm{C} 13$ to $\mathrm{C} 15)$ and the $8^{\circ}$ rotation of the side chain of Y466 indicates a correlated motion in line with

8 the strong electronic coupling observed by quantum chemistry between the substrate and Y466. In

9 order to reflect the optimum $\mathrm{pH}$ of FAP in solution ( $\mathrm{pH} 8.5$; Ref. (4)) and to overcome thermal

10 activation barriers, a FAP crystal was soaked at $\mathrm{pH} 8.5$ and illuminated at $150 \mathrm{~K}$ ('150 K light').

11 Interestingly, after modeling alkane and $\mathrm{CO}_{2}$ molecule, the residual $F_{\mathrm{o}}-F_{\mathrm{c}}$ electron density map

12 showed a positive density with a triangular shape close to $\mathrm{C} 432$, which we tentatively attribute to

13 bicarbonate with 30\% occupancy (Fig. 4D and fig. S14).

Time-resolved serial femtosecond crystallography of $C v$ FAP

16 We investigated the structural changes occurring in FAP after photoexcitation at room temperature

17 by a time-resolved SFX (TR-SFX) experiment (35) using a pump-probe scheme. Time-resolved

18 fluorescence spectroscopy on FAP microcrystals established that the kinetics of fET are the same

19 as in solution (supplementary text S5, fig. S15). For TR-SFX at the Linac Coherent Light Source

20 (LCLS), FAP microcrystals were photoexcited by picosecond $400 \mathrm{~nm}$ pump pulses and probed by

21 femtosecond XFEL pulses after pump-probe delays of 20 ps, 900 ps, $300 \mathrm{~ns}$, and $2 \mu$ s to cover time

22 scales on which $\mathrm{FAD}$ reduction and $\mathrm{FAD}^{\bullet-}$ reoxidation occur. The 'SFX dark' state structure 
1 mentioned above, was determined from the data in the absence of a pump laser pulse. Structural

2 changes after photoexcitation were visualized as positive and negative peaks in difference Fourier

3 electron density maps calculated between the light and dark data sets $\left(F_{\text {obs }}{ }^{\text {light } \_t}{ }^{t}-F_{\text {obs }}{ }^{\text {dark}}\right.$; Fig. 5A)

4 at $2.2 \AA$ resolution. The most prominent difference electron density peaks at all four time points

5 are at the active site, with the highest negative peak ( -5.5 to $-10.1 \sigma$, depending on the time point)

6 at the position of the carboxyl group of the substrate, showing that light-induced decarboxylation

7 occurred (Fig. 5A). At 900 ps, decarboxylation has occurred to a considerable extent, in line with

8 the 270 ps time constant determined by multi-scale time-resolved IR spectroscopy (Fig. 2C-E). At

$9300 \mathrm{~ns}$ and $2 \mu \mathrm{s}$, a strong negative peak is observed at Wat1 ( -5.8 , and $-6.2 \sigma$, respectively, Fig.

10 5A), but not on Wat2. We note the absence of positive difference electron density peaks associated

11 with the photodissociated $\mathrm{CO}_{2}$ in the vicinity of the substrate carboxyl group. A possible reason

12 could be the small initial displacement of cleaved $\mathrm{CO}_{2}$ relative to its position in the fatty acid, in

13 line with the structure determined based on an illuminated cryo-cooled crystal ' $100 \mathrm{~K}$ light' (see

14 fig. S13C for comparison). It is conceivable that the positive difference densities close to C432 in

15 the $300 \mathrm{~ns}$ and $2 \mu \mathrm{s}$ data sets (Fig. 5A) correspond to the feature(s) observed in the data obtained

16 from cryo-cooled crystals illuminated at $150 \mathrm{~K}$ (Fig. 5B and Fig. 4D), tentatively assigned to a

17 bicarbonate (fig. S14). In the SFX data, attempts to fit unambiguously this positive difference

18 density with a bicarbonate, a $\mathrm{CO}_{2}$ molecule or a mixture thereof remained unsatisfactory. The

19 absence of significant difference electron density peaks at the FAD at all four time points suggests

20 that the isoalloxazine ring does not undergo significant light-induced conformational changes.

22 Quantum chemistry study of $C v$ FAP 
1 We performed quantum-chemistry calculations for the decarboxylation reaction in the active site

2 of FAP, considering electronic states relevant for flavin photoexcitation, electron transfer,

3 decarboxylation and alkane formation as well as effects of flavin bending (supplementary text S6).

4 To perform the reaction pathway calculations, a large active site model (consisting of 272 atoms;

5 fig. S16) was prepared using the coordinates of the high-resolution crystal structure of the dark

6 state (Fig. 1D). Surprisingly, computations indicated multiple routes to form an alkane in the FAP

7 active site as summarized in Fig. 6.

8 Common to all routes, photoexcitation triggers charge transfer (CT) from the substrate to 9 the flavin. Interactions with two water molecules stabilize the anionic carboxylate substrate (1)

10 (Fig. 6A). Removing these water molecules from the active site or rearranging Wat1 to form a 11 hydrogen bond with flavin substantially decreases the energy of the CT state, i.e. of flavin radical 12 anion and fatty acid radical (table S6). After Wat1 rearrangement, the CT energy is $15 \mathrm{kcal} / \mathrm{mol}$ 13 lower than the excited-flavin energy (table S6). Concomitantly, electronic coupling between the 14 flavin and substrate increases almost three fold reaching $47 \mathrm{meV}$ (table S7), favoring transfer of an 15 electron from the carboxylate. The formed carboxylic radical undergoes a barrierless 16 decarboxylation (fig. S17) affording the alkyl radical (3). The energy of the alkyl radical 17 intermediate is $52 \mathrm{kcal} / \mathrm{mol}$ above the dark state (1), which corresponds to more than $80 \%$ of the 18 photon energy (Fig. 6B). $\mathrm{CO}_{2}$ dissociation increases the distance separation in the radical pair, and 19 accordingly the electronic coupling between the alkyl and flavin radicals is only $7 \mathrm{meV}$ (table S7).

From the alkyl radical (3), the alkane product (6/6a) can be formed via several reaction

21 pathways (I-III in Fig. 6). The chemical changes characterizing pathways I-III are summarized in

22 Fig. 6C. Hydrogen atom transfer (HAT) from a nearby residue has been discussed previously (4, 23 24). Our results indicate strong interactions with the Y466 phenolic side chain (electronic coupling 
$1142 \mathrm{meV}$; table S7) that may facilitate migration of the alkyl radical towards Y466 and C432. The

2 HAT reaction from C432 (pathway I in Fig. 6) proceeds via an energy barrier (9 kcal/mol; fig.

3 S18) and leads to a Cys-radical state (4) with a $15 \mathrm{kcal} / \mathrm{mol}$ lower energy than that of the alkyl

4 radical intermediate (3). Re-oxidation of the flavin by C432 (5) further reduces the energy by 3

$5 \mathrm{kcal} / \mathrm{mol}$ (Fig. 6B). The resulting thiolate anion is stabilized by a hydrogen bond with positively

6 charged R451, however proton transfer from R451 to C432 in (5) along the hydrogen bond yielding

7 (5a) does not further reduce the energy (table S5). Hence, it is likely that C432 re-protonation

8 proceeds by a different mechanism (see below).

Alternatively to the HAT reaction involving C432, a proton can be transferred from Wat2 which stabilizes the alkyl radical (3). The shift of the negative charge from the flavin to the alkane by bET coupled to proton transfer from Wat2 leads to a transient formation of a hydroxyl anion

12 which either interacts with $\mathrm{CO}_{2}$ forming bicarbonate (6a) directly (pathway III in Fig. 6), or it 13 deprotonates R451 (5b) (pathway II in Fig. 6). The presence of $\mathrm{CO}_{2}$ and water molecules in the 14 active site, in particular Wat1, allows R451 re-protonation concomitant with bicarbonate formation 15 (6), even after the alkane is formed, with a small activation energy (4 kcal/mol; fig. S19). The 16 bicarbonate product derived from Wat 1 (6) is $10 \mathrm{kcal} / \mathrm{mol}$ lower than the bicarbonate originating 17 from Wat2 (6a), rendering pathway II more energetically favorable than pathway III (Fig. 6B). 18 Energy lowering via bicarbonate formation may also further stabilize product (5) of the HAT reaction (pathway I): in the first step the thiolate C432 obtains a proton from $\mathrm{R} 451$ and/or Wat1 20 and in the second step, bicarbonate is formed from $\mathrm{CO}_{2}$ and $\mathrm{OH}^{-}$derived from Wat1 (6).

In view of the experimentally observed red-shifted re-oxidized flavin intermediate, we have 22 compared the flavin excitation energy of the species resulting from the bET reaction and that of 23 the initial dark state (Fig. 6B, table S8). Our computations suggest that the red shift can be 
1 explained by formation of various species in which the initial negative charge of the deprotonated

2 carboxylate (1) is either neutralized by formation of $\mathrm{CO}_{2}$ and deprotonated $\mathrm{R} 451$ (5) or shifted

3 away from the flavin by formation of the anionic bicarbonate (6) and (6a) (fig. S20). Additionally,

4 a red shifted spectrum is caused by hydrogen-bonding interactions of Wat 1 with flavin in (5a).

5 Consistent with the X-ray structures, all active-site models contain the butterfly bending

6 conformation of the flavin isoalloxazine ring. Flavin bending persisted during geometry

7 optimization of the FAP active site models (Fig. 6A) in contrast to the essentially planar optimized

8 geometry of the oxidized and semi-reduced forms obtained in computations of flavins (36). The

9 significant bending angle in the optimized FAP active-site structures is consistent with the notion

10 that interactions with the protein modulate flavin bending (37). As previously discussed, this

11 bending biases the energy levels of the flavin, favoring flavin reduction (36) and decreasing the

12 excitation energy (table S9) with possible functional implications. In particular, bending diminishes

13 the vertical electron affinity even more than the excited-flavin energy (fig. S21), and by this,

14 preferentially reduces the CT-state energy which should facilitate fET. Thus, flavin bending

15 explains the strongly red-shifted $(20 \mathrm{~nm})$ ground-state FAD absorption spectrum of FAP in the

16 dark state compared to free FAD or most flavoproteins allowing photo-excitation and facile fET

17 up to as far as $530 \mathrm{~nm}$. Importantly, this is well into the so-called 'green gap' (between 500 and

$18600 \mathrm{~nm})$ in the absorption spectra of chlorophylls that dominate the absorption of algae, thus

19 enhancing the net light-harvesting capacities of FAP.

21 Role of conserved amino acids in the FAP active site 
1 The active site of $C v \mathrm{FAP}$ contains three residues (Y466, C432 and R451) that are strictly conserved

2 and specific to FAPs compared to other GMC oxidoreductases (fig. S23). Y466 and C432 were

3 previously considered as hydrogen atom donor to the alkyl radical $\mathrm{R}^{\bullet}$ (followed by bET from

$4 \mathrm{FAD}^{\bullet-}$ to the tyrosyl or cysteinyl radical) or, alternatively, proton donor in a proton-coupled back-

5 ET from $\mathrm{FAD}^{\bullet-}$ to $\mathrm{R}^{\bullet}(4,24)$. Our quantum chemical calculations additionally suggest $\mathrm{R} 451$ as a

6 potential proton donor. In order to identify the proton donor experimentally, we performed FTIR;

7 in particular, we analyzed possible contributions from deprotonated forms of these three residues

8 in the cryo-trapped red-shifted intermediate. Light-induced FTIR difference spectra of $C v$ FAP

9 recorded at $100 \mathrm{~K}$ show only a shift of a $v(\mathrm{~S}-\mathrm{H})$ IR mode of cysteine, and no changes in $\mathrm{D}_{2} \mathrm{O}$

10 compared to $\mathrm{H}_{2} \mathrm{O}$ that could be assigned to tyrosine vibrations, which does not support

11 deprotonation of either of the two residues under conditions where the flavin red shift was observed

12 (Fig. 4A panel $v i$ and supplementary text S2). However, a strong negative band was observed at

$131606 \mathrm{~cm}^{-1}$ in FTIR difference spectra recorded in $\mathrm{D}_{2} \mathrm{O}$ (Fig. 4A vii). In ${ }^{15} \mathrm{~N}$-labeled FAP samples,

14 this band downshifted to $1597 \mathrm{~cm}^{-1}$ (Fig. 4A, viii). Both observations support assignment of the

15 band to the guanidium IR mode of an arginine side-chain. Absence of a positive counterpart of this

16 band after illumination is indicative of arginine deprotonation in the FAD $\mathrm{RS}_{\text {state }} 100 \mathrm{~K}$

17 (supplementary text S2).

To gain further insight into the role of Y466, C432 and R451, we prepared Y466F, C432S,

19 R451A and R451K mutants, performed activity measurements and extensive structural and

20 spectroscopic characterization (UV-Vis absorption spectra are shown in fig. S24). Mutation of

21 Y466 to phenylalanine affected the catalytic activity only slightly (Fig. 7A), in line with a previous

22 report (24). The kinetics of both fET to $\mathrm{FAD}$ and bET from $\mathrm{FAD}^{\bullet-}$ do not differ much from WT

$23 C \nu \mathrm{FAP}$ (fig. S25C). Furthermore, almost identical FTIR difference spectra were obtained with WT 
$1 \quad \mathrm{CvFAP}$ and the Y466F mutant (fig. S26A). Altogether, these results suggest that Y466 is not

2 directly participating in any PT/HAT or ET step in the FAP photocycle, and its role is only briefly

3 discussed in the supplementary text S4.

Mutation of $\mathrm{C} 432$ to serine (a much poorer proton and hydrogen atom donor) strongly

5 affected the catalytic activity (Fig. 7A), as reported earlier by Heyes and coworkers, who

6 considered it indicative of HAT from C432 to the alkyl radical $\mathrm{R}^{\bullet}$ in the WT protein (24). In

7 contrast to these authors, we detected a low but significant catalytic activity ( 10\% of WT) for

8 the $\mathrm{C} 432 \mathrm{~S}$ mutant. Since impairment of the catalytic activity by a point mutation may result from

9 structural changes rather than suppression of a direct function of the replaced residue, we examined

10 the $\mathrm{C} 432 \mathrm{~S}$ mutant protein in more detail. The dark state crystal structure of $\mathrm{C} 432 \mathrm{~S}$ is highly similar

11 to WT except for a new water molecule Wat3, interacting with S432 (2.7 $⿱$ A) and the O1 oxygen

12 atom of the fatty acid carboxylate $(2.5 \AA)$, which is rotated by $\sim 42^{\circ}$ (Fig. 7D). The distance of the

13 carboxylate $\mathrm{O} 2$ to the flavin N5 increases from $4.0 \AA$ in WT to $5.0 \AA$.

The time-resolved fluorescence signal of $\mathrm{C} 432 \mathrm{~S}$ resembles that of WT after the consumption of native substrate(s), however, the fluorescence decay is slightly $(\sim 10 \%)$ accelerated

(Fig. 7B), indicating that the signal reflects mostly intrinsic ${ }^{1} \mathrm{FAD}^{*}$ decay (mostly due to

17 intersystem crossing (ISC) (4)), with only a small contribution of competing fET to ${ }^{1} \mathrm{FAD} *$. The

18 intrinsic fET rate is about ten times slower than ${ }^{1} \mathrm{FAD}^{*}$ decay in this mutant (see supplementary

19 text S3). Transient absorption signals on the sub-ms time scale were hence dominated by the triplet

20 (fig. S25F) and the formation of $\mathrm{FAD}_{\mathrm{RS}}$ could not be resolved. There was, however, a small ( $15 \%$

21 of WT) long-lived absorption change at $515 \mathrm{~nm}$ (where the transient flavin red-shift is most

22 prominent in the WT). About two thirds of it decayed in a few milliseconds, i.e., with similar

23 kinetics as FADRs in WT (see upper inset of fig. S25F or Fig. 3D in (24)). With these indirect 
1 indications of $F A D_{R S}$ formation in $\mathrm{C} 432 \mathrm{~S}$, we attempted to accumulate this species at a cryogenic

2 temperature. Illumination of the sample at $200 \mathrm{~K}$ yielded a spectrum with similar shape (but lower

3 amplitude) as the FAD ${ }_{\text {RS }}$ spectrum obtained with WT FAP (Fig. 7C).

When R451 was mutated to alanine, the enzymatic activity was completely abolished (Fig.

5 7A), no fET was observed (fig. S25B); in fact the structure shows that the fatty acid is oriented

6 very differently than in the WT (see Fig. S26D and supplementary text S3 and S4 for details).

When R451 was replaced by lysine (which is also positively charged), the catalytic activity amounted to $\sim 45 \%$ of WT (Fig. 7A). The fluorescence decay (Fig. 7B) was distinctly faster ( 4.5 ns) than in WT without substrate ( $\sim 6.5 \mathrm{~ns})$, consistent with fET occurring for $\sim 30 \%$ of the excited flavins. Reoxidation of $\mathrm{FAD}^{\bullet-}$ and formation of $\mathrm{FAD}_{\mathrm{RS}}$ was clearly resolved and found to be markedly faster ( $\sim 30 \mathrm{~ns}$; fig. S25D) than in WT ( 100 ns; Fig. 3B, fig. S10A; supplementary text

12 S3 and S4). The acceleration of the (proton-coupled) bET would be consistent with lysine being a 13 better proton donor than arginine (solution $\mathrm{p} K_{\mathrm{a}}$ of Lys is 10.7 vs. 12.1 for $\mathrm{Arg}$ ).

To better understand how R451 affects the active site architecture, we determined the crystal structure of the R451K mutant in its dark-state at $100 \mathrm{~K}$ (Fig. 7D and fig. S26C). The R451K mutant structure differs significantly from WT: while a new water molecule mimics the

$17 \mathrm{NH}_{2}$ group of $\mathrm{R} 451$ and thereby retains the interaction with the $\mathrm{O} 2$ oxygen atom of the fatty acid carboxylate $(2.5 \AA)$, the interaction between $\mathrm{K} 451$ and the fatty acid $\mathrm{O} 1$ oxygen atom induces a $19 \sim 54^{\circ}$ rotation of the carboxylate and an increase by $0.6 \AA$ of the distance of the fatty acid $\mathrm{O} 2$ to the 20 flavin N5 compared to WT. This creates space for a new water molecule Wat3, akin to the situation

21 in $\mathrm{C} 432 \mathrm{~S}$, located between the fatty acid O1 atom $(2.4 \AA)$ and K451 (2.8 $\AA$ ). The close distance

22 of Wat3 and C432 induces a flip of the amino acid stretch T430 to G435, pushing C432 out of the 
1 active site and bringing in an additional water molecule. These large structural changes may explain

2 the very different FTIR spectra obtained with the R451K mutant compared to WT (fig. S26B).

\section{Reaction cycle of FAP}

5 In line with the strict conservation of $\mathrm{C} 432$ and R451 in FAP sequences, even conservative

6 substitutions of these residues result in drastic reductions in catalytic activity, in both cases by

7 strongly reducing fET. Unexpectedly, the R451K and C432S mutants share significant structural

8 modifications with respect to the WT: the presence of a new water molecule, Wat 3 close to the

9 fatty acid carboxylate, a rotation of the carboxylate by $\sim 50^{\circ}$, a significant elongation of the distance

10 of the fatty acid carboxylate to the flavin N5, as well as small changes in Wat1 location. Quantum

11 chemistry calculations show that in the C432S mutant the CT energy increases by $0.2 \mathrm{eV}$ and the

12 electronic coupling reduces five-fold, which is consistent with a much slower observed fET (see

13 supplementary text 6.7 and fig. S22).

14 These mutants provide important insights into the molecular constraints affording FAP 15 activity: the active site of the WT enzyme is arranged such as to optimize the configuration of the

16 fatty acid carboxylate for fET. Each carboxylate oxygen atom interacts with catalytically important

17 groups (O1: Wat1, O2: R451 and Wat2) while avoiding an inactivating bidentate interaction with $18 \mathrm{R} 451$.

Three possible routes towards alkane formation in FAP were suggested by quantum

20 chemistry (Fig. 6C). Our experimental findings allow assessing the suggested pathways. Pathway

21 I, involving a HAT mechanism to reduce the alkyl radical, as also suggested previously $(4,24)$, is 
1 chemically plausible. However, a number of experimental findings argue against it. First, the

$2 \mathrm{C} 432 \mathrm{~S}$ mutant retains significant enzymatic activity, suggesting that C432 is not essential for

3 catalysis. Second, in the FAD $\mathrm{RS}_{\mathrm{S}}$ state only a shift of a thiol S-H vibration is observed by FTIR but

4 no cysteine deprotonation. Third, despite the fact that C432 is rotated out of the active site in the

$5 \mathrm{R} 451 \mathrm{~K}$ mutant, this variant is quite active. While it is possible that $\mathrm{C} 432$ rotates back into the active

6 site upon changes in the water structure following $\mathrm{CO}_{2}$ cleavage, this would not explain the three-

7 fold faster bET in R451K. Taken together, reaction pathway I (Fig. 6C) seems unlikely, although

8 we cannot rule out that the C432S mutant uses a different mechanism than the WT enzyme. The

9 observation of residual electron density consistent with bicarbonate close to C432 suggests that

$10 \mathrm{C} 432$ may stabilize reaction products such as $\mathrm{CO}_{2}$ and/or bicarbonate away from the original

11 position of the substrate carboxylate.

The other two pathways proceed via PCET (Fig. 6C). Pathway III implies a catalytic role

of Wat2 in bicarbonate formation. This mechanism is unlikely, as no changes were observed for

14 Wat2 in the electron density maps derived by TR-SFX. In contrast, $300 \mathrm{~ns}$ after photoexcitation a

15 significant loss of electron density of Wat 1 was observed, supporting Pathway II. With the cleaved

$16 \mathrm{CO}_{2}$ present in the active site, as evidenced by cryocrystallography and IR spectroscopy, Wat 2 can

17 serve as a proton donor. The transiently deprotonated R451 activates Wat 1 , resulting in bicarbonate

18 formation in less than $100 \mathrm{~ns}$. This transformation of $\mathrm{CO}_{2}$ to bicarbonate is orders of magnitude

19 faster than in solution (tens of seconds (38)) indicating a strong catalytic effect.

R451's role as transient proton donor was a priori unexpected since proton transfers from

21 and to arginine residues are rare $(39,40)$ due to their relatively high $\mathrm{p} K_{\mathrm{a}}$ values. In FAP, the strong

22 basicity of $\mathrm{OH}^{-}$formed from Wat2 by proton-coupled ET to the alkyl radical may allow proton

23 transfer from R451. In addition to this catalytic function, R451 is crucial for the FAP active site 
1 architecture by precisely positioning and orienting the fatty acid head group with respect to FAD

2 and stabilizing the carboxylate in the catalytically active deprotonated form.

Figure 8 and its legend summarize our comprehensive understanding of the strikingly

4 complex cycle. By combining results obtained by a multitude of experimental techniques and

5 computations, we provide a detailed mechanistic description of the evolution of the reactant (fatty

6 acid) to the products (alka(e)ne and $\mathrm{CO}_{2}$ ) and the role of the protein moiety, involving a proton

7 coupled electron transfer mechanism. We demonstrate partly unexpected structural and dynamic

8 properties of FAP, including features that have not been observed in other flavoproteins and other

9 enzymatic reactions in general. Understanding these catalytic features is an important step in

10 incorporating FAP into the green chemistry toolkit.

\section{Materials and Methods Summary}

The FAP used in all experiments corresponds to residues 76-654 of the full-length $C v$ FAP

14 (or single mutants thereof obtained by site-directed mutagenesis). WT $C \nu$ FAP and $C \nu$ FAP mutants

15 expressed in E. coli and purified were studied by X-ray crystallography (static and TR-SFX),

16 spectroscopy in solution (FTIR, TR-IR on ps to $\mu$ s timescales, time-resolved fluorescence

17 spectroscopy on ps to ns timescales, transient absorption spectroscopy on ns to ms timescales, and

18 ultrafast visible absorption and fluorescence spectroscopy) and spectroscopy on single crystals

19 (UV-Vis and Raman). Activity assays were based on quantification of hydrocarbons formed (by

20 gas chromatography coupled to mass spectrometry) or $\mathrm{CO}_{2}$ released (by membrane inlet mass

21 spectrometry). Computational studies involved molecular dynamics simulations and quantum

22 chemistry calculations on $C v \mathrm{FAP}$ and multiple alignments of GMC oxidoreductases protein

23 sequences. Detailed Materials and Methods are available in Supplementary Materials. 


\section{References and Notes}

3 1. L. O. Björn, "Photoactive proteins" in Photobiology: The Science of Light and Life

4 (Springer-Verlag, New York, ed. 3, 2015), pp. 139-150.

5 2. A. Sancar, Mechanisms of DNA Repair by Photolyase and Excision Nuclease (Nobel

6 Lecture). Angew. Chem. Int. Ed. 55, 8502-8527 (2016).

7 3. M. Gabruk, B. Mysliwa-Kurdziel, Light-Dependent Protochlorophyllide Oxidoreductase:

8 Phylogeny, Regulation, and Catalytic Properties. Biochemistry 54, 5255-5262 (2015).

9 4. D. Sorigué et al., An algal photoenzyme converts fatty acids to hydrocarbons. Science $10 \quad 357,903-907$ (2017).

115 D. Zhong, Ultrafast catalytic processes in enzymes. Curr. Opin. Chem. Biol. 11, 174-181 12 (2007).

13 6. M. J. Maul et al., Crystal Structure and Mechanism of a DNA (6-4) Photolyase. Angew. 14 Chem. Int. Ed. 47, 10076-10080 (2008).

15 7. P. Müller et al., Discovery and functional analysis of a 4th electron-transferring 16 tryptophan conserved exclusively in animal cryptochromes and (6-4) photolyases. Chem.

17 Commun. (Camb.) 51, 15502-15505 (2015).

18 8. A. Mees et al., Crystal structure of a photolyase bound to a CPD-like DNA lesion after in 19 situ repair. Science 306, 1789-1793 (2004).

209 D. J. Heyes et al., Ultrafast enzymatic reaction dynamics in protochlorophyllide 21 oxidoreductase. Nat. Struct. Mol. Biol. 10, 491-492 (2003).

22 10. N. S. Scrutton, M. L. Groot, D. J. Heyes, Excited state dynamics and catalytic mechanism 23 of the light-driven enzyme protochlorophyllide oxidoreductase. Phys. Chem. Chem. Phys. 14, 
2 11. D. J. Heyes et al., Excited-state charge separation in the photochemical mechanism of the

3 light-driven enzyme protochlorophyllide oxidoreductase. Angew. Chem. Int. Ed. 54, 1512-1515 $4 \quad$ (2015).

5 12. S. Zhang et al., Structural basis for enzymatic photocatalysis in chlorophyll biosynthesis.

$6 \quad$ Nature 574, 722-725 (2019).

7 13. C.-S. Dong et al., Crystal structures of cyanobacterial light-dependent

8 protochlorophyllide oxidoreductase. Proc. Natl. Acad. Sci. U.S.A. 117, 8455-8461 (2020).

9 14. M. A. Emmanuel, N. R. Greenberg, D. G. Oblinsky, T. K. Hyster, Accessing non-natural 10 reactivity by irradiating nicotinamide-dependent enzymes with light. Nature 540, 414-417 11 (2016).

12 15. N. S. Scrutton, Enzymes make light work of hydrocarbon production. Science 357, 872$13873(2017)$.

14 16. T. Courtney, A. Deiters, Recent advances in the optical control of protein function 15 through genetic code expansion. Curr. Opin. Chem. Biol. 46, 99-107 (2018).

16 17. S. Moulin et al., Continuous photoproduction of hydrocarbon drop-in fuel by microbial 17 cell factories. Sci. Rep. 9 (2019).

18 18. W. Zhang et al., Hydrocarbon Synthesis via Photoenzymatic Decarboxylation of 19 Carboxylic Acids. J. Am. Chem. Soc. 141, 3116-3120 (2019).

20 19. M. Amer et al., Low carbon strategies for sustainable bio-alkane gas production and 21 renewable energy. Energy Environ. Sci. (2020).

22 20. N. A. Herman, W. Zhang, Enzymes for fatty acid-based hydrocarbon biosynthesis. Curr. 23 Opin. Chem. Biol. 35, 22-28 (2016). 
1 21. J. Xu et al., Light-Driven Kinetic Resolution of $\alpha$-Functionalized Carboxylic Acids

2 Enabled by an Engineered Fatty Acid Photodecarboxylase. Angew. Chem. Int. Ed. 58, 8474-8478

3 (2019).

4 22. D. Sorigué et al., Microalgae Synthesize Hydrocarbons from Long-Chain Fatty Acids via 5 a Light-Dependent Pathway. Plant Physiol. 171, 2393-2405 (2016).

6 23. S. Moulin et al., "Fatty acid photodecarboxylase is an ancient photoenzyme responsible

7 for hydrocarbon formation in the thylakoid membranes of algae".

8 https://www.biorxiv.org/content/10.1101/2020.06.23.166330v1

9 24. D. J. Heyes et al., On the photochemical mechanism of light-driven fatty acid

10 photodecarboxylase. ACS Catal. (2020).

11 25. T. Senda, M. Senda, S. Kimura, T. Ishida, Redox control of protein conformation in

12 flavoproteins. Antioxid. Redox Signal. 11, 1741-1766 (2009).

13 26. A. K. Røhr, H.-P. Hersleth, K. K. Andersson, Tracking flavin conformations in protein

14 crystal structures with Raman spectroscopy and QM/MM calculations. Angew. Chem. Int. Ed. 49, $15 \quad 2324-2327$ (2010).

16 27. A. Aleksandrov, A Molecular Mechanics Model for Flavins. J. Comput. Chem. 40, 2834$172842(2019)$.

18 28. H. N. Chapman et al., Femtosecond X-ray protein nanocrystallography. Nature 470, 73$1977(2011)$

20 29. K. Hirata et al., Determination of damage-free crystal structure of an X-ray-sensitive 21 protein using an XFEL. Nat. Methods 11, 734-736 (2014).

22 30. S. P. Laptenok et al., Ultrafast real-time visualization of active site flexibility of 23 flavoenzyme thymidylate synthase ThyX. Proc. Natl. Acad. Sci. U.S.A. 110, 8924-8929 (2013). 
1 31. L. H. Jones, E. McLaren, Infrared Absorption Spectra of SO2 and CO2 in Aqueous

2 Solution. J. Chem. Phys. 28, 995-995 (1958).

3 32. L. Antonucci et al., Arbitrary-detuning asynchronous optical sampling with amplified

$4 \quad$ laser systems. Opt. Express 23, 27931-27940 (2015).

5 33. X. Solinas, L. Antonucci, A. Bonvalet, M. Joffre, Multiscale control and rapid scanning

6 of time delays ranging from picosecond to millisecond. Opt Express. 25, 17811-17819 (2017).

7 34. B. A. Diner, D. A. Force, D. W. Randall, R. D. Britt, Hydrogen Bonding, Solvent

8 Exchange, and Coupled Proton and Electron Transfer in the Oxidation and Reduction of Redox-

9 Active Tyrosine YZ in Mn-Depleted Core Complexes of Photosystem II. Biochemistry 37,

10 17931-17943(1998).

11 35. J.-P. Colletier, G. Schirò, M. Weik, in X-ray Free Electron Lasers: A Revolution in

12 Structural Biology, S. Boutet, P. Fromme, M. S. Hunter, Eds. (Springer International Publishing,

13 Cham, 2018), pp. 331-356.

14 36. J. D. Walsh, A.-F. Miller, Flavin reduction potential tuning by substitution and bending.

15 J. Mol. Struct. THEOCHEM. 623, 185-195 (2003).

16 37. B. W. Lennon, C. H. Williams, M. L. Ludwig, Crystal structure of reduced thioredoxin

17 reductase from Escherichia coli: Structural flexibility in the isoalloxazine ring of the flavin

18 adenine dinucleotide cofactor. Protein Sci. 8, 2366-2379 (1999).

19 38. N. McCann et al., Kinetics and Mechanism of Carbamate Formation from CO2(aq),

20 Carbonate Species, and Monoethanolamine in Aqueous Solution. J. Phys. Chem. A. 113, 5022-

$215029(2009)$.

22 39. Y. Xiao et al., Role of Arginine-82 in Fast Proton Release during the Bacteriorhodopsin

23 Photocycle: A Time-Resolved FT-IR Study of Purple Membranes Containing 15N-Labeled 
1 Arginine. Biochemistry 43, 12809-12818 (2004).

2 40. P. J. Silva et al., A tale of two acids: when arginine is a more appropriate acid than $\mathrm{H} 3 \mathrm{O}+$.

3 J. Phys. Chem. B. 114, 8994-9001 (2010).

4 41. A. Burlacot, F. Burlacot, Y. Li-Beisson, G. Peltier, Membrane Inlet Mass Spectrometry:

5 A Powerful Tool for Algal Research. Front. Plant Sci. 11 (2020).

6 42. M. Roth et al., FIP: a highly automated beamline for multiwavelength anomalous

7 diffraction experiments. Acta Crystallogr. D Biol. Crystallogr. 58, 805-814 (2002).

8 43. D. de Sanctis et al., ID29: a high-intensity highly automated ESRF beamline for

9 macromolecular crystallography experiments exploiting anomalous scattering. J Synchrotron

10 Rad. 19, 455-461 (2012).

11 44. D. von Stetten et al., In crystallo optical spectroscopy (icOS) as a complementary tool on

12 the macromolecular crystallography beamlines of the ESRF. Acta Crystallogr. D Biol.

13 Crystallogr. 71, 15-26(2015).

14 45. A. Vagin, A. Teplyakov, Molecular replacement with MOLREP. Acta Crystallogr. D

15 Biol. Crystallogr. 66, 22-25 (2010).

16 46. G. N. Murshudov, A. A. Vagin, E. J. Dodson, Refinement of macromolecular structures

17 by the maximum-likelihood method. Acta Cryst. D Biol. Cristallogr. 53, 240-55 (1997).

18 47. P. Emsley, K. Cowtan, Coot: model-building tools for molecular graphics. Acta

19 Crystallogr. D Biol Crystallogr. 60, 2126-32 (2004).

20 48. J. McGeehan et al., Colouring cryo-cooled crystals: online microspectrophotometry. $J$.

21 Synchrotron Rad. 16, 163-172 (2009).

22 49. D. von Stetten et al., Online Raman spectroscopy for structural biology on beamline ID29

23 of the ESRF. J. Struct. Biol. 200, 124-127 (2017). 
1 50. O. B. Zeldin, M. Gerstel, E. F. Garman, RADDOSE-3D: time- and space-resolved

2 modelling of dose in macromolecular crystallography. J. Appl. Cryst. 46, 1225-1230 (2013).

3 51. D. von Stetten et al., ID30A-3 (MASSIF-3) - a beamline for macromolecular

4 crystallography at the ESRF with a small intense beam. J Synchrotron Rad. 27, 844-851 (2020).

5 52. J. Sanchez-Weatherby et al., Improving diffraction by humidity control: a novel device

6 compatible with X-ray beamlines. Acta Crystallogr. D Biol. Crystallogr. 65, 1237-1246 (2009).

7 53. A. Espagne, M. Byrdin, A. P. M. Eker, K. Brettel, Very Fast Product Release and

8 Catalytic Turnover of DNA Photolyase. ChemBioChem. 10, 1777-1780 (2009).

9 54. M. Byrdin et al., Use of ruthenium dyes for subnanosecond detector fidelity testing in real

10 time transient absorption. Rev. Sci. Instrum. 80, 043102 (2009).

11 55. P. Müller et al., ATP Binding Turns Plant Cryptochrome Into an Efficient Natural

12 Photoswitch. Sci. Rep. 4, 5175 (2014).

13 56. L. Nag et al., Identification of the TyrOH •+ Radical Cation in the Flavoenzyme TrmFO.

$14 \quad J$. Am. Chem. Soc. 139, 11500-11505 (2017).

15 57. S. P. Laptenok, P. Nuernberger, A. Lukacs, M. H. Vos, Subpicosecond Kerr-gate

16 spectrofluorometry. Methods Mol. Biol. 1076, 321-336 (2014).

17 58. M. A. Marangoni et al., Narrow-bandwidth picosecond pulses by spectral compression of

18 femtosecond pulses in second-order nonlinear crystals. Opt Express. 15, 8884-8891 (2007).

19 59. J. Bredenbeck, J. Helbing, P. Hamm, Continuous scanning from picoseconds to

20 microseconds in time resolved linear and nonlinear spectroscopy. Rev. Sci. Instrum. 75, 4462-

214466 (2004).

22 60. L. Antonucci, X. Solinas, A. Bonvalet, M. Joffre, Asynchronous optical sampling with

23 arbitrary detuning between laser repetition rates. Opt Express. 20, 17928-17937 (2012). 
1 61. K. F. Lee, P. Nuernberger, A. Bonvalet, M. Joffre, Removing cross-phase modulation

2 from midinfrared chirped-pulse upconversion spectra. Opt Express. 17, 18738-18744 (2009).

3 62. J. J. Snellenburg et al., Glotaran: A Java-Based Graphical User Interface for the R

$4 \quad$ Package TIMP. J. Stat. Soft. 49 (2012).

5 63. D. Flot et al., The ID23-2 structural biology microfocus beamline at the ESRF. $J$.

6 Synchrotron Rad. 17, 107-118 (2010).

7 64. U. Zander et al., MeshAndCollect: an automated multi-crystal data-collection workflow

8 for synchrotron macromolecular crystallography beamlines. Acta Crystallogr. D. Biol.

$9 \quad$ Cristallogr. 71, 2328-2343 (2015).

10 65. L. Lomb et al., An anti-settling sample delivery instrument for serial femtosecond

11 crystallography. J. Appl Crystallogr. 45, 674-678 (2012).

12 66. U. Weierstall, J. C. Spence, R. B. Doak, Injector for scattering measurements on fully

13 solvated biospecies. Rev. Sci. Instrum. 83, 035108 (2012).

14 67. M. Liang et al., The Coherent X-ray Imaging instrument at the Linac Coherent Light

15 Source. J. Synchrotron Rad. 22, 514-519 (2015).

16 68. M. L. Grünbein et al., Illumination guidelines for ultrafast pump-probe experiments by

17 serial femtosecond crystallography. Nat. Methods (2020).

18 69. G. Blaj et al., X-ray detectors at the Linac Coherent Light Source. J. Synchrotron Rad. 22,

$19 \quad 577-583(2015)$.

20 70. L. Foucar et al., CASS-CFEL-ASG software suite. Comput. Phys. Commun. 183, 2207-

$212213(2012)$.

22 71. N. Coquelle et al., Raster-scanning serial protein crystallography using micro- and nano-

23 focused synchrotron beams. Acta Crystallog. D. Biol. Cristallgr. 71, 1184-1196 (2015). 
1 72. T. White, Processing serial crystallography data with CrystFEL: a step-by-step guide.

$2 \quad$ Acta Crystallogr. Section D. 75 (2019).

3 73. Y. Gevorkov et al., XGANDALF - extended gradient descent algorithm for lattice

4 finding. Acta crystallogr. A. Found Adv. 75, 694-704 (2019).

5 74. K. Nass et al., Protein structure determination by single-wavelength anomalous

6 diffraction phasing of X-ray free-electron laser data. IUCrJ. 3, 180-91 (2016).

7 75. A. J. McCoy et al., Phaser crystallographic software. J. Appl. Crystallogr. 40, 658-674

8 (2007).

9 76. W. L. DeLano, The PyMOL Molecular Graphics System. San Carlos, CA, USA: DeLano

10 Scientific (2002).

11 77. T. Ursby, D. Bourgeois, Improved Estimation of Structure-Factor Difference Amplitudes

12 from Poorly Accurate Data. Acta Crystallogr. A. 53, 564-575 (1997).

13 78. A. T. Brünger et al., Crystallography \& NMR System: A New Software Suite for

14 Macromolecular Structure Determination. Acta Crystallogr. D. 54, 905-921 (1998).

15 79. C. Wickstrand, R. Dods, A. Royant, R. Neutze, Bacteriorhodopsin: Would the real

16 structural intermediates please stand up? Biochim. Biophys. Acta 1850, 536-553 (2015).

17 80. K. Nass et al., Structural dynamics in proteins induced by and probed with X-ray free-

18 electron laser pulses. Nat. Commun. 11, 1814 (2020).

19 81. T. J. Dolinsky, J. E. Nielsen, J. A. McCammon, N. A. Baker, PDB2PQR: an automated

20 pipeline for the setup of Poisson-Boltzmann electrostatics calculations. Nucleic Acids Res. 32,

21 W665-W667 (2004).

22 82. M. H. M. Olsson, C. R. Søndergaard, M. Rostkowski, J. H. Jensen, PROPKA3:

23 Consistent Treatment of Internal and Surface Residues in Empirical pKa Predictions. J. Chem. 
2 83. T. Darden, in Computational biochemistry and biophysics (Oren M. Becker, Alexander D.

3 MacKerell Jr., Benoit Roux, Masakatsu Watanabe., 2001).

4 84. N. Foloppe, A. D. M. Jr, All-atom empirical force field for nucleic acids: I. Parameter

5 optimization based on small molecule and condensed phase macromolecular target data. $J$.

6 Comput. Chem. 21, 86-104 (2000).

7 85. A. D. MacKerell et al., All-atom empirical potential for molecular modeling and

8 dynamics studies of proteins. J. Phys. Chem. B. 102, 3586-3616 (1998).

9 86. W. L. Jorgensen et al., Comparison of simple potential functions for simulating liquid 10 water. J. Chem. Phys. 79, 926-935 (1983).

11 87. E. Neria, S. Fischer, M. Karplus, Simulation of activation free energies in molecular 12 systems. J. Chem. Phys. 105, 1902-1921 (1996).

13 88. J. C. Phillips et al., Scalable molecular dynamics with NAMD. J. Comput. Chem. 26, $14 \quad 1781-1802(2005)$.

15 89. Firefly program version 8.0, (available at

16 http://classic.chem.msu.su/gran/gamess/index.html).

17 90. M. W. Schmidt et al., General atomic and molecular electronic structure system. $J$.

18 Comput. Chem. 14, 1347-1363 (1993).

19 91. A. D. Becke, Density-functional thermochemistry. III. The role of exact exchange. $J$.

20 Chem. Phys. 98, 5648-5652(1993).

21 92. C. Lee, W. Yang, R. Parr, Development of the Colle-Salvetti correlation-energy formula 22 into a functional of the electron density. Phys. Rev., B Condens. Matter. 37, 785-789 (1988).

23 93. S. Grimme, J. Antony, S. Ehrlich, H. Krieg, A consistent and accurate ab initio 
1 parametrization of density functional dispersion correction (DFT-D) for the 94 elements H-Pu. $J$.

2 Chem. Phys. 132, 154104 (2010).

3 94. D. E. Woon, T. H. D. Jr, Gaussian basis sets for use in correlated molecular calculations.

4 V. Core-valence basis sets for boron through neon. J. Chem. Phys. 103, 4572 (1998).

5 95. A. A. Granovsky, Extended multi-configuration quasi-degenerate perturbation theory: the

6 new approach to multi-state multi-reference perturbation theory. J. Chem. Phys. 134, 214113

7 (2011).

$896 . \quad$ H. A. Witek, Y.-K. Choe, J. P. Finley, K. Hirao, Intruder state avoidance multireference

9 Møller-Plesset perturbation theory. J. Comput. Chem. 23, 957-965 (2002).

$1097 . \quad$ V. Veryazov, P. Å. Malmqvist, B. O. Roos, How to select active space for

11 multiconfigurational quantum chemistry? Int. J. Quantum Chem. 111, 3329-3338 (2011).

12 98. A. Udvarhelyi, M. Olivucci, T. Domratcheva, Role of the Molecular Environment in

13 Flavoprotein Color and Redox Tuning: QM Cluster versus QM/MM Modeling. J. Chem. Theory

14 Comput. 11, 3878-3894 (2015).

15 99. A. R. Moughal Shahi, T. Domratcheva, Challenges in Computing Electron-Transfer

16 Energies of DNA Repair Using Hybrid QM/MM Models. J. Chem. Theory Comput. 9, 4644-

$174652(2013)$.

18 100. I. A. Solov'yov, T. Domratcheva, A. R. Moughal Shahi, K. Schulten, Decrypting

19 cryptochrome: revealing the molecular identity of the photoactivation reaction. J. Am. Chem. Soc.

20 134, 18046-18052 (2012).

21 101. T. Domratcheva, Neutral histidine and photoinduced electron transfer in DNA

22 photolyases. J. Am. Chem. Soc. 133, 18172-18182 (2011).

23 102. G. Nass Kovacs et al., Three-dimensional view of ultrafast dynamics in photoexcited 
1 bacteriorhodopsin. Nat. Commun. 10, 3177 (2019).

2 103. R. J. Cave, M. D. Newton, Generalization of the Mulliken-Hush treatment for the

3 calculation of electron transfer matrix elements. Chem. Phys. Lett. 249, 15-19 (1996).

4 104. R. C. Edgar, MUSCLE: multiple sequence alignment with high accuracy and high

5 throughput. Nucleic Acids Res. 32, 1792-1797 (2004).

6 105. M. Gouy, S. Guindon, O. Gascuel, SeaView version 4: A multiplatform graphical user

7 interface for sequence alignment and phylogenetic tree building. Mol. Biol. Evol. 27, 221-224

8 (2010).

9 106. V. SYu, N. N. Kalnin, Quantitative IR spectrophotometry of peptide compounds in water 10 (H2O) solutions. I. Spectral parameters of amino acid residue absorption bands. Biopolymers 30, $11 \quad 1243-1257(1990)$.

12 107. M. Falk, A. G. Miller, Infrared spectrum of carbon dioxide in aqueous solution. Vib.

13 Spectrosc. 4, 105-108 (1992).

14 108. A. R. Davis, B. G. Oliver, A vibrational-spectroscopic study of the species present in the 15 CO2-H2O system. J. Solution Chem. 1, 329-339 (1972).

16 109. R. Hienerwadel, C. Berthomieu, Bicarbonate binding to the non-heme iron of

17 photosystem II investigated by Fourier transform infrared difference spectroscopy and 13C-

18 labeled bicarbonate. Biochemistry 34, 16288-16297 (1995).

19 110. H. Susi, D. M. Byler, W. V. Gerasimowicz, Vibrational analysis of amino acids: cysteine,

20 serine, $\beta$-chloroalanine. J. Mol. Struct. 102, 63-79 (1983).

21 111. H. Kandori et al., Cysteine $\mathrm{S}-\mathrm{H}$ as a Hydrogen-Bonding Probe in Proteins. J. Am. Chem.

22 Soc. 120, 5828-5829 (1998).

23 112. G. Dollinger et al., Fourier transform infrared difference spectroscopy of 
1 bacteriorhodopsin and its photoproducts regenerated with deuterated tyrosine. Biochemistry $\mathbf{2 5}$,

$2 \quad 6524-6533(1986)$.

3 113. Y. N. Chirgadze, O. V. Fedorov, N. P. Trushina, Estimation of amino acid residue side-

4 chain absorption in the infrared spectra of protein solutions in heavy water. Biopolymers 14, 679-

$5694(1975)$.

6 114. M. S. Braiman, D. M. Briercheck, K. M. Kriger, Modeling vibrational spectra of amino

7 acid side chains in proteins: Effects of protonation state, counterion, and solvent on arginine C-N

8 stretch frequencies. J. Phys. Chem. B. 103, 4744-4750 (1999).

9 115. P. F. Heelis, The photophysical and photochemical properties of flavins (isoalloxazines).

10 Chem. Soc. Rev. 11, 15-39 (1982).

11 116. A. Mozzarelli, G. L. Rossi, Protein function in the crystal. Annu. Rev. Biophys. Biomol.

12 Struct. 25, 343-365 (1996).

13 117. J. Woodhouse et al., Photoswitching mechanism of a fluorescent protein revealed by

14 time-resolved crystallography and transient absorption spectroscopy. Nat. Commun. 11, 741

15 (2020).

16 118. M. R. Jackson et al., A preference for edgewise interactions between aromatic rings and

17 carboxylate anions: the biological relevance of anion-quadrupole interactions. J. Phys. Chem. B.

$18 \quad \mathbf{1 1 1}, 8242-8249$ (2007).

19 1119. V. Kane Dickson, L. Pedi, S. B. Long, Structure and insights into the function of a

$20 \mathrm{Ca}(2+)$-activated $\mathrm{Cl}(-)$ channel. Nature 516, 213-218 (2014).

21 120. E. Park, E. B. Campbell, R. MacKinnon, Structure of a CLC chloride ion channel by

22 cryo-electron microscopy. Nature 541, 500-505 (2017).

23 121. P. A. Karplus, K. Diederichs, Linking Crystallographic Model and Data Quality. Science 


\section{Acknowledgements}

4 We thank Joyce Woodhouse for help with microcrystallization, Lucas Uriarte for help with

5 spectroscopy experiments on crystals and Gabriela Nass Kovacs for preparation of injection

6 conditions, Miroslaw Tarnawski for FAP stability measurements, Filipe Maia for helpful

7 discussions and uploading the SFX data to CXIDB.ORG and Michel Philibert and the GRAP

8 platform for custom manufacturing of scientific equipment. The IBS acknowledges integration into

9 the Interdisciplinary Research Institute of Grenoble (IRIG, CEA) and financial support by the CEA,

10 the CNRS and the UGA. The authors thank the staff of beamline ID-23-2 from the ESRF, Grenoble,

11 France. The present work has benefited from the platform of Biophysics of I2BC supported by

12 French Infrastructure for Integrated Structural Biology (FRISBI) ANR-10-INBS-05 and from the

13 platform Heliobiotec (BIAM). Funding: ANR SNAPsHOTs to F.B., M.W., P.M., K.B., M.H.V.,

14 P.A., R.H., and C.B.; ANR Photoalkane to G.P.; ANR SignalBioRNJ to Y.L.-B.; ANR BioXFEL

15 to M.W.; ERC Consolidator Grant STepLADDER (724362) to T.R.M.B., Chinese Scholarship

16 Council fellowship to B.Z. MENESR - Univ. Grenoble Alpes fellowship to K.H. Author

17 contributions: F.B., M.W., P.A., P.M., I.S., K.B., M.H.V., C.B., T.D. designed and organized the 18 project.

J.-P.C., M.J., T.R.M.B., A.R. supervised parts of the project. D.S., S. Blangy, P.S., S. Cuiné., B.L.

20 S.M., Y.L.-B., G.P., E.H. and F.B. analyzed FAP sequences, generated mutants, expressed and

21 purified the FAPs and performed activity assays. D.S., S. Blangy, E.H. and P.A. produced crystals

22 for synchrotron experiments, P.A., G.G., A.R., D.N., P.L., I.S. and D.S. acquired synchrotron 23 diffraction data, G.G., P.A. and A.R. performed in crystallo optical spectroscopies and P.A, I.S., 
1 G.G., P.L. and A.R. interpreted data. D.S., R.H. and C.B. built the setup for cryogenic light-induced

2 FTIR difference spectroscopy, acquired and analyzed the data. A. Bonvalet, A. Benachir, D.S.

3 optimized and performed multi-scale TR-IR experiments, L.A., X.S, A. Bonvalet, M.J. conceived

4 and developed multi-scale TR-IR spectroscopy and adapted for FAP experiments, A. Bonvalet.,

5 L.A., M.J., M.H.V. analyzed TR-IR data. M.H.V. performed and analyzed femtosecond pump-

6 probe absorption and fluorescence spectroscopy. B.Z., M.H.V. performed and analyzed

7 picosecond-pump power dependence spectroscopy. A.A. performed molecular dynamics

8 simulations. D.S., S. Blangy and K.H. produced microcrystals for SFX and K.H., J.-P.C. and M.W

9 tested their diffraction quality at the ESRF. M.S., K.H. and D.S. carried out time-resolved emission

10 and absorption spectroscopy on microcrystals. T.RMB, M.C., S. Boutet, J.-P.C., N.C., R.B.D.,

11 L.F., A.G., M.L.G., K.H., M.H., M.K., T.J.L., G.S., I.S., R.L.S., M.W. carried out SFX

12 experiments. R.L.S., R.B.D., M.L.G., and M.K. performed sample injection. S.Carbajo, G.S. and

13 M.C. carried out laser work at the LCLS. S. Boutet and T.J.L. prepared and performed SFX data

14 collection. L.F., M.H., N.C., J.P.C. and T.R.M.B. performed online SFX data analysis. K.H., N.C.,

15 J.-P.C., A.G., T.R.M.B. carried out offline SFX data processing. K.H., J.-P.C., N.C., M.W.,

16 T.R.M.B., I.S. analysed SFX data. M.B., K.B. and P.M. built the setups for ps-ns time-resolved

17 fluorescence and ns-ms transient absorption spectroscopy. D.S., P.S., B.Z., P.M. and K.B.

18 performed the measurements. P.M. and K.B. analyzed the data. T.D. performed quantum chemistry

19 calculations and provided mechanistic insight. F.B., P.M., K.B., M.H.V., I.S., P.A., T.D., M.W.,

20 C.B., D.S. wrote the paper. The paper was discussed with all authors. Competing interests: The

21 authors declare no competing interests. Data and materials availability: All data are available in

22 the manuscript, the supplementary materials, or at publicly accessible repositories. Atomic

23 coordinates of protein structures have been deposited in the Protein Data Bank under accession no. 
1 6YRU, 6YRV, 6YRX, 6YRZ, 6YS1, 6YS2, 6ZH7, 7AV4. SFX diffraction images of FAP

2 microcrystals have been deposited in the Coherent X-ray Imaging Data Bank website (CXIDB)

3 with accession ID 177 (http://cxidb.org/id-177.html).

4

\section{Supplementary Materials:}

$6 \quad$ Materials and Methods

$7 \quad$ Supplementary text S1-S6

$8 \quad$ Figures S1-S31

9 Tables S1-S11

10 References (41-122)

11 

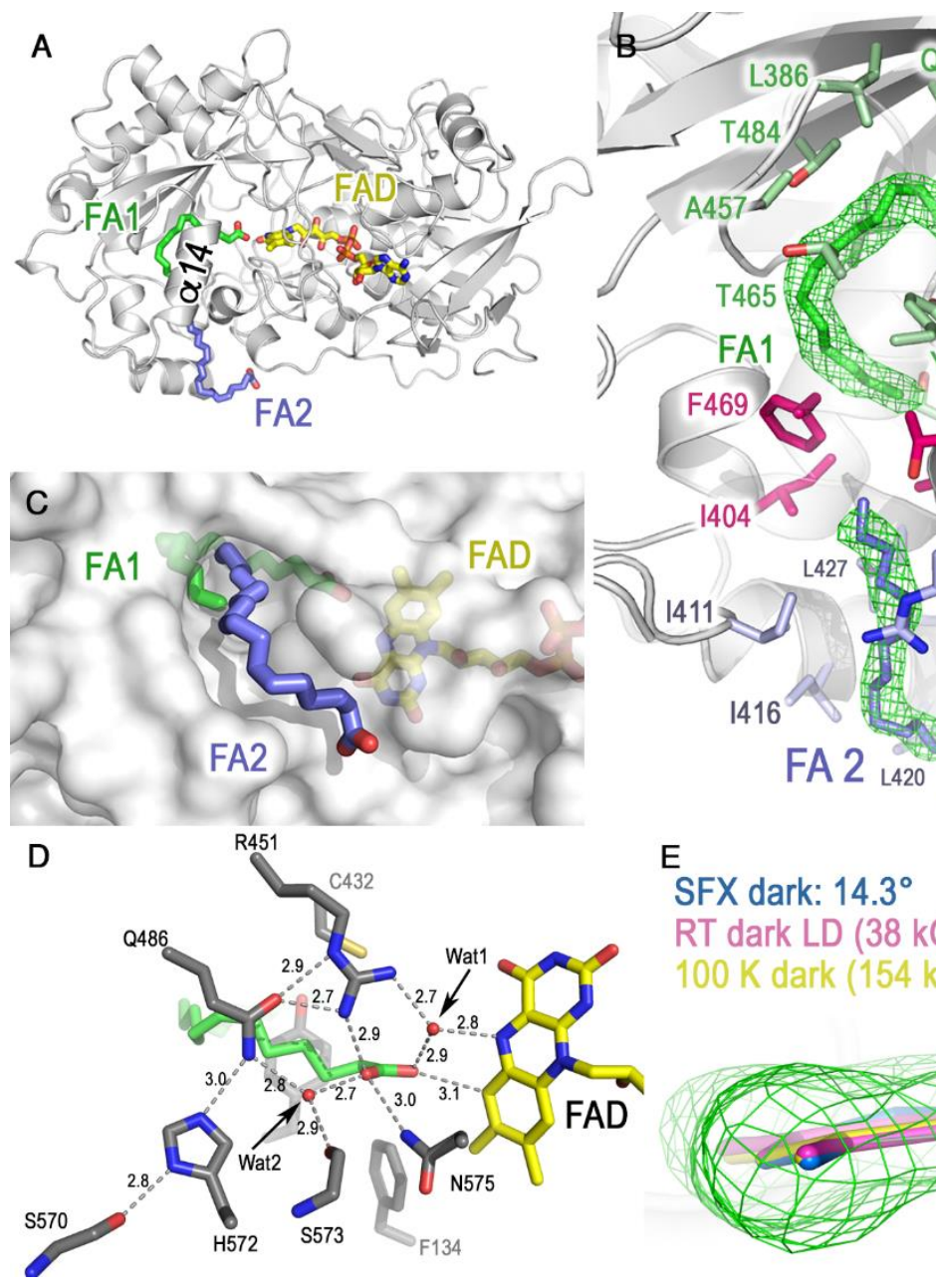

SFX dark: $14.3^{\circ}$

RT dark LD (38 kGy): $13.7^{\circ}$

$100 \mathrm{~K}$ dark (154 kGy): $17.4^{\circ}$

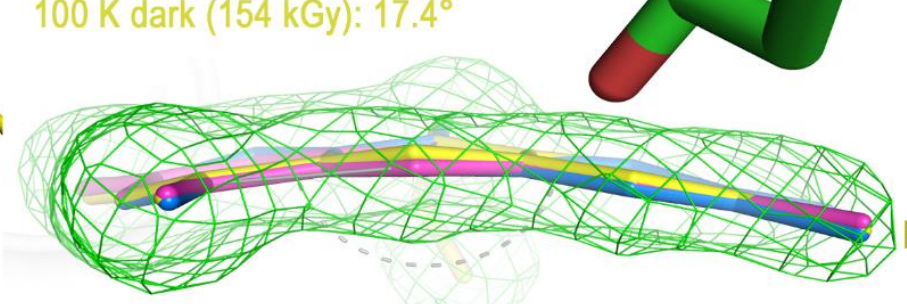


1 Fig. 1. High resolution crystal structures of $C v$ FAP. (A) Structure of Chlorella variabilis FAP

$2(\mathrm{CvFAP})$ determined from synchrotron data at $100 \mathrm{~K}$ (' $100 \mathrm{~K}$ dark'), including the FAD cofactor

3 and two C18 fatty acid substrates (FA1 and FA2). (B) Binding of the two substrate molecules. The

4 'omit' electron density map (5.0 $\sigma$ contour level) is shown as a green mesh, and amino acid side

5 chains in a radius of $4 \AA$ around the binding site are shown as sticks (green: active site, blue:

6 secondary binding site, purple: between sites. (C) Position of the peripheral substrate (FA2); partly

7 obstructing the channel leading to the active site tunnel. (D) Close-up view of the catalytic site,

8 showing the water molecules Wat1 and Wat2 and the interactions with FAD (yellow sticks) and

9 the substrate in the active site FA1 (green sticks). Distances are indicated in Ångstrom. The shortest

10 distance between the substrate and the FAD cofactor (carboxylate O1-isoalloxazine C6) is $3.1 \AA$.

11 The tail of the peripheral substrate points towards the entrance of a tunnel leading to the active site

12 that is lined by A128, T131, I404 and F469. (E) Superposition of the FAD isoalloxazine rings from

13 the SFX dark structure (blue; molecule A), and the synchrotron structures (pink: 'RT dark low-

14 dose', yellow: ' $100 \mathrm{~K}$ dark'). The SFX $F_{\text {obs }}-F_{\text {calc }}$ omit map at $3 \sigma$ (green) is overlaid, and the FAD

15 bending angles are indicated. 
A

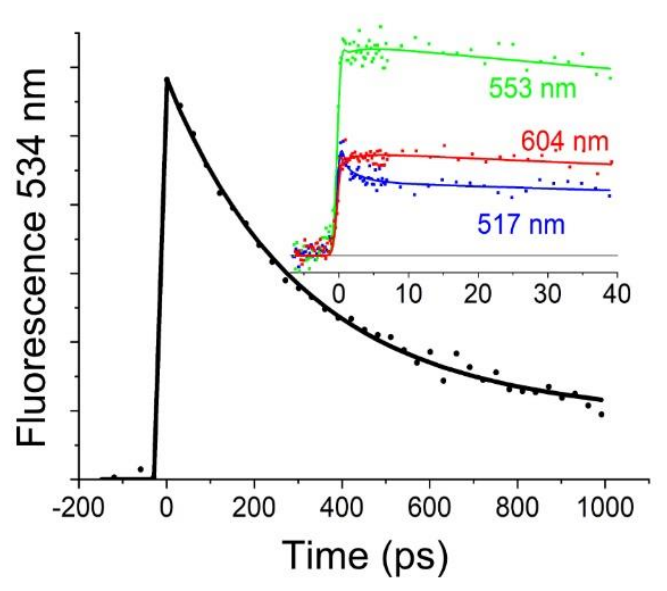

C

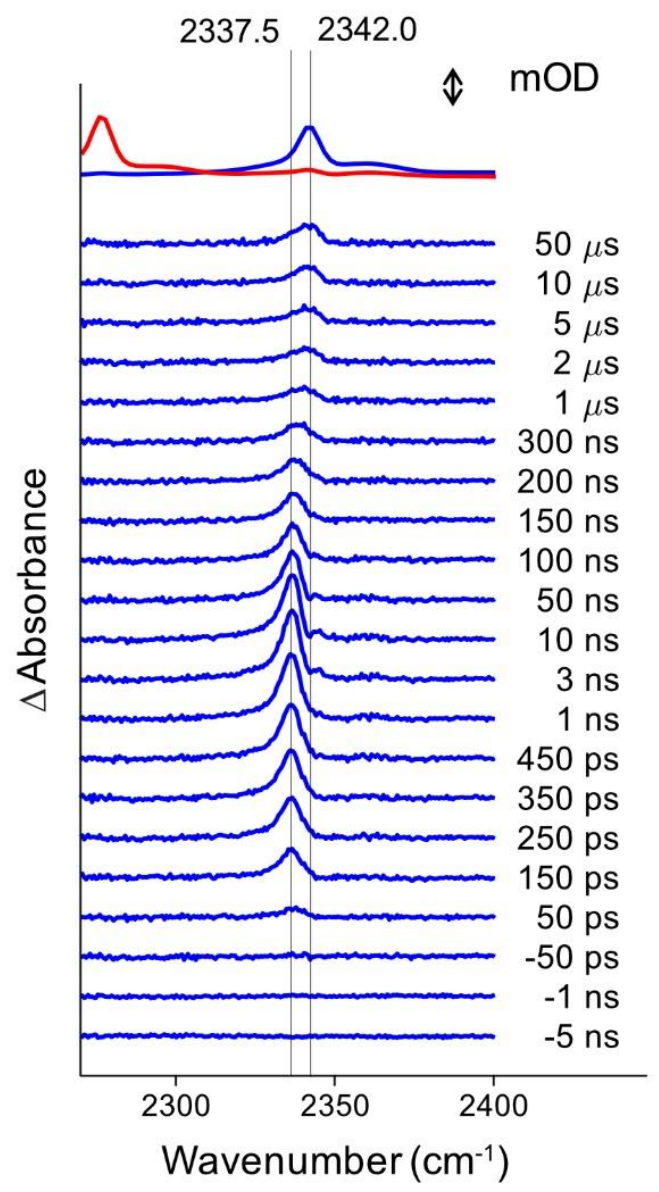

B

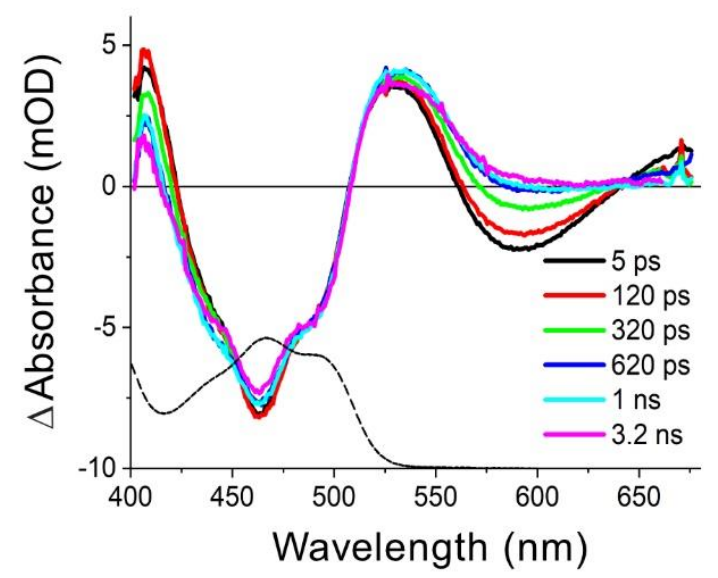

D

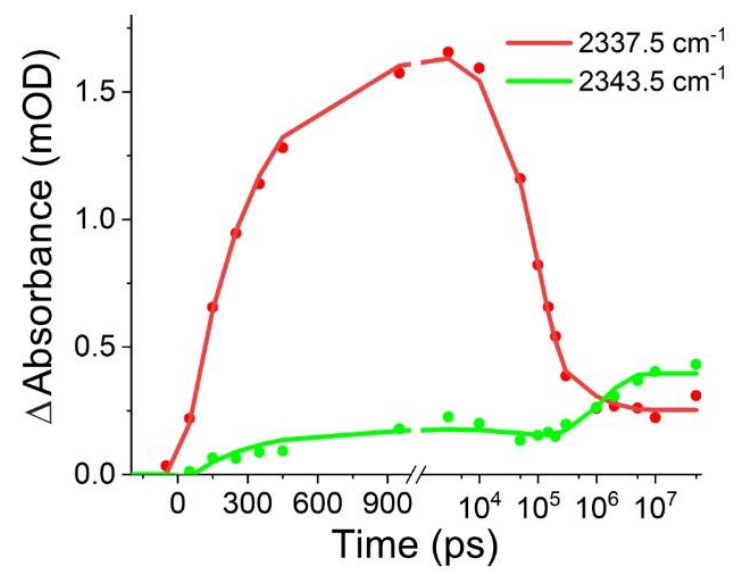

E

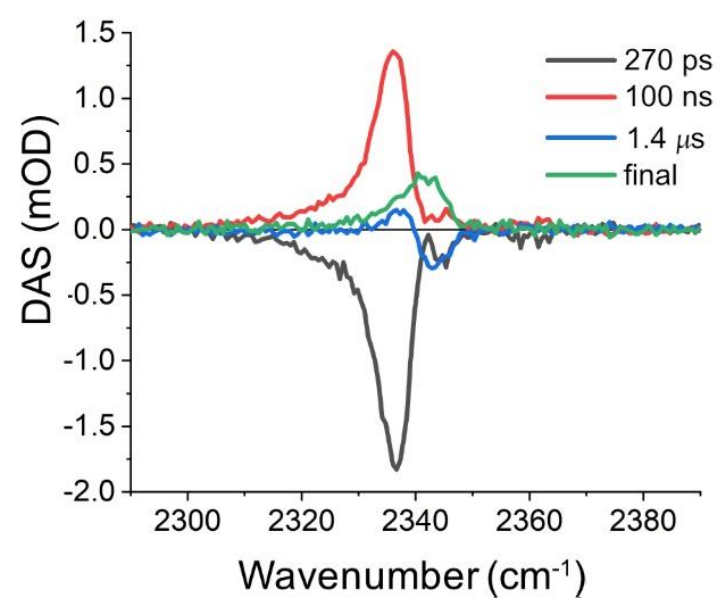


1 Fig. 2. Time-resolved infrared and UV-visible spectroscopies of $C v F A P$. (A) Fluorescence

2 kinetics reflecting ${ }^{1} \mathrm{FAD}^{*}$ decay. The solid line is a fit with time constants of $300 \mathrm{ps}(85 \%)$ and 5

3 ns (the latter time constant was imposed according to (Ref. (4)). The inset shows an additional $\sim 2$

4 ps wavelength-dependent phase reflecting redshifting of the fluorescence spectrum due to excited

5 state relaxation. (B) Transient visible absorption spectra at different delay times in the same time

6 domain. The negative absorption features reflect bleaching of the FAD ox resting state $(<525 \mathrm{~nm}$,

7 the black dashed line is the ground state absorption spectrum) and stimulated emission (550 - 650

$8 \mathrm{~nm}$ ). Full transient fluorescence spectra and global analysis of the transient absorption spectra are

9 shown in fig. S8. (C) Transient infrared spectra in the $\mathrm{CO}_{2}$ spectral region on the picosecond-

10 microsecond time scale. The vertical lines are guides for the eye and correspond to the maximum

11 of released $\mathrm{CO}_{2}$ in the protein and to the known maximum for $\mathrm{CO}_{2}$ in aqueous solution, $2342 \mathrm{~cm}^{-}$

$12^{1}$ (31). The upper traces correspond to independent steady-state $298 \mathrm{~K}$ light-induced FTIR

13 difference spectra with ${ }^{12} \mathrm{C}$-palmitate and $1-{ }^{13} \mathrm{C}$-palmitate substrates (black trace and red trace

14 respectively). (D) Kinetics at frequencies close to the initial and final maxima of released $\mathrm{CO}_{2}$. The

15 lines are the result of a global fit. The timescale is linear up to $1 \mathrm{~ns}$ and logarithmic thereafter. (E)

16 Decay Associated Spectra (DAS) corresponding to a global fit of the data with three exponential

17 phases (time constants of the fit are indicated) and a constant phase. 
A

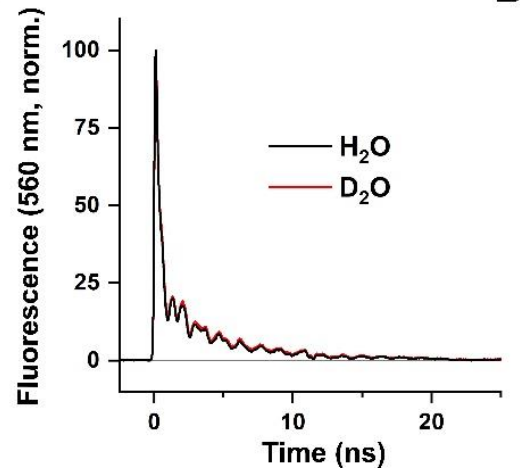

B

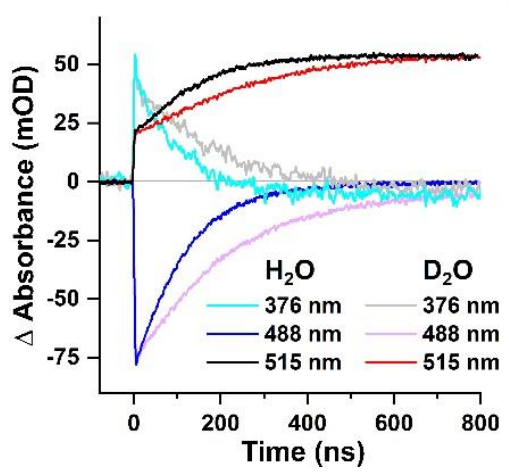

C

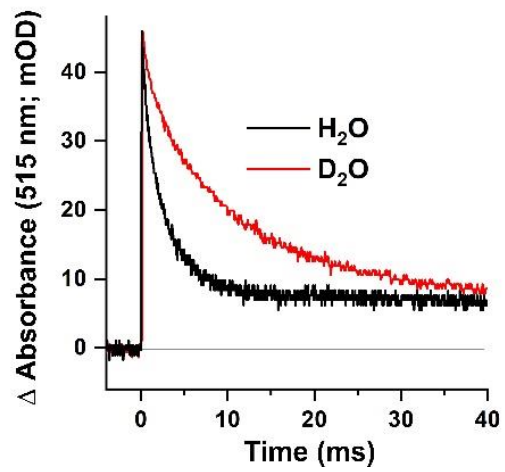

3 Fig. 3. Effects of $\mathrm{H}_{2} \mathrm{O} / \mathrm{D}_{2} \mathrm{O}$ exchange on time-resolved fluorescence and $\mathrm{UV}$-visible

4 spectroscopy of $\boldsymbol{C v F A P}$. (A) Normalized signals of time-resolved fluorescence of ${ }^{1} \mathrm{FAD} *$ at 560

$5 \mathrm{~nm}$ in $\mathrm{H}_{2} \mathrm{O}$ and $\mathrm{D}_{2} \mathrm{O}$ buffers. (B) Effect of $\mathrm{H}_{2} \mathrm{O}$ to $\mathrm{D}_{2} \mathrm{O}$ exchange on the kinetics of $\mathrm{FAD}^{\bullet-}$

6 reoxidation to $\mathrm{FAD}_{\mathrm{RS}}$ followed by transient absorption spectroscopy at selected wavelengths on a

7 sub-microsecond timescale. (C) Effect of $\mathrm{H}_{2} \mathrm{O}$ to $\mathrm{D}_{2} \mathrm{O}$ buffer exchange on the decay of FAD

8 measured at $515 \mathrm{~nm}$ on the millisecond timescale.

9 

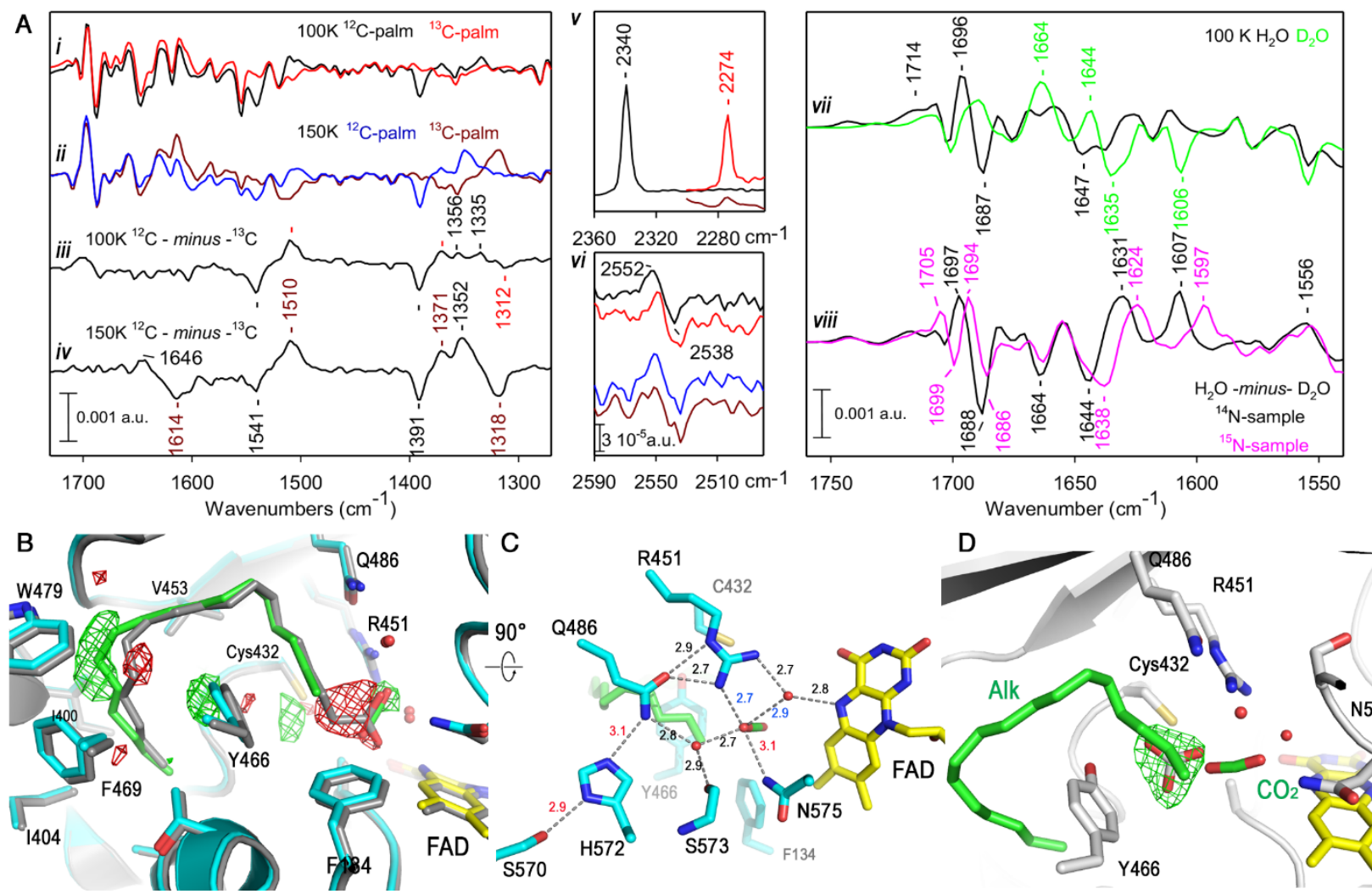
1 Fig. 4. Characterization of the $C v F A P$ red-shifted intermediate at cryogenic temperatures

2 (A) Light-induced FTIR difference spectra recorded from FAP solutions $i$ ) and ii) and 3 corresponding ${ }^{12} \mathrm{C}$ - minus $-{ }^{13} \mathrm{C} »$ difference spectra iii) and iv). The $2360-2260 \mathrm{~cm}^{-1}$ and 2590

$4 \quad-2490 \mathrm{~cm}^{-1}$ regions of the spectra in $i$ ) and $i$ ) are shown in panels $v$ ) and $v i$ ). For the spectrum in

5 black, the FTIR spectrometer was continuously purged with $\mathrm{N}_{2}$ to avoid contamination by gaseous

$6 \mathrm{CO}_{2}$ that might originate from a dry air purge. This was not the case for spectra recorded from FAP

7 samples with $1-{ }^{13} \mathrm{C}$-palmitate; these were cut off above $2300 \mathrm{~cm}^{-1}$ to avoid bands from gaseous

$8 \mathrm{CO}_{2}$; vii) overlay of the spectra recorded at $100 \mathrm{~K}$ from FAP with ${ }^{12} \mathrm{C}$-palmitate in $\mathrm{H}_{2} \mathrm{O}$ (black) and

$9 \quad$ in $\mathrm{D}_{2} \mathrm{O}$ (green); viii) $\ll \mathrm{H}_{2} \mathrm{O}-$ minus $-\mathrm{D}_{2} \mathrm{O} »$ difference spectra calculated for ${ }^{14} \mathrm{~N}$ and ${ }^{15} \mathrm{~N}$-labelled

10 FAP samples. (B) Experimental difference density map ( $F_{\text {light }}-F_{\text {dark }}, 100 \mathrm{~K}$ data $)$ contoured at $+/-$

$114 \sigma$ around the active site substrate, superimposed on the refined structures of the dark state (gray)

12 and the red-shifted form (cyan, with FAD in yellow and alkane/ $\mathrm{CO}_{2}$ in green). The cleavage of the

13 C1-C2 bond is clearly visible. (C) Details of the active site of $\mathrm{FAP}_{\mathrm{RS}}$ formed upon illumination

14 (UV-Vis spectra in fig. S12). All distances shorter than $3.2 \AA$ are labeled, except the fatty acid O1

15 - FAD C6 distance, which increases from $3.1 \AA$ in the dark to $3.6 \AA$ upon illumination. (D) $F_{\mathrm{o}}-F_{\mathrm{c}}$

16 electron density omit map (3.5 $\sigma)$ derived from crystals kept at $150 \mathrm{~K}$ and $\mathrm{pH} 8.5$, showing positive

17 difference electron density next to $\mathrm{C} 432$, consistent with a bicarbonate. Its potential interactions

18 with the environment are shown in fig. S14. Uncleaved fatty acid ( 30\% occupancy) is omitted for 19 clarity. 
A

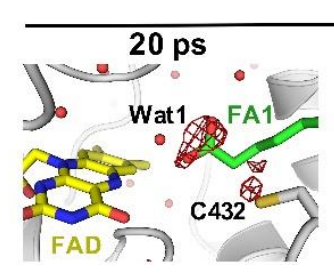

TR-SFX

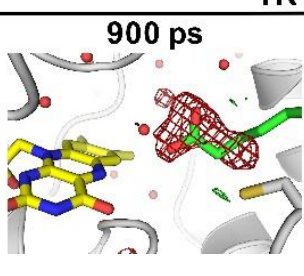

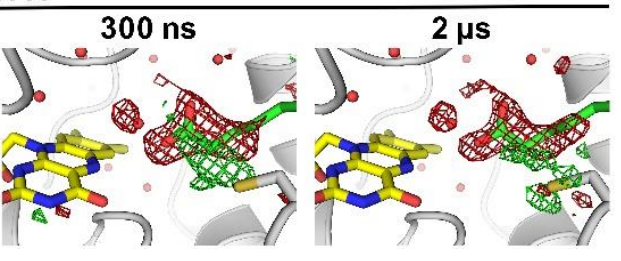

B

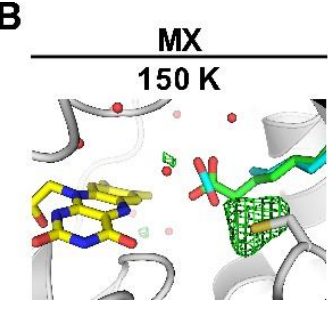

3 Fig. 5. Time-dependent changes in the $C v$ FAP active site followed by TR-SFX.

4 (A)Locally averaged q-weighted difference Fourier maps calculated between the SFX light and

5 dark data sets $\left(F_{\mathrm{obs}}{ }^{\text {light } \_\Delta t}-F_{\mathrm{obs}}{ }^{\text {dark }}\right.$; with $\left.\Delta t=20 \mathrm{ps}, 900 \mathrm{ps}, 300 \mathrm{~ns}, 2 \mu \mathrm{s}\right)$. The $2.2 \AA$ resolution maps are shown at $+4 \sigma$ (green) and $-4 \sigma$ (red). The SFX dark state model of molecule B is overlaid, with FAD in yellow, fatty acid in green, and the protein in grey. The q-weighted difference maps

8 for molecules $\mathrm{A}$ and $\mathrm{B}$, i.e., without local averaging, are shown in fig. S31. (B) $F_{\mathrm{obs}}-F_{\text {calc }}$ electron

9 density (contoured at $3.5 \sigma$ ) of Fig. 3E, shown in a different orientation, which features unmodeled 10 positive electron density next to C432 that is reminiscent of a bicarbonate (Fig. 4D, fig. S14, see text), in a location similar to where positive difference density is present in the time-resolved maps

12 at $300 \mathrm{~ns}$ and $2 \mu \mathrm{s}$. 
A
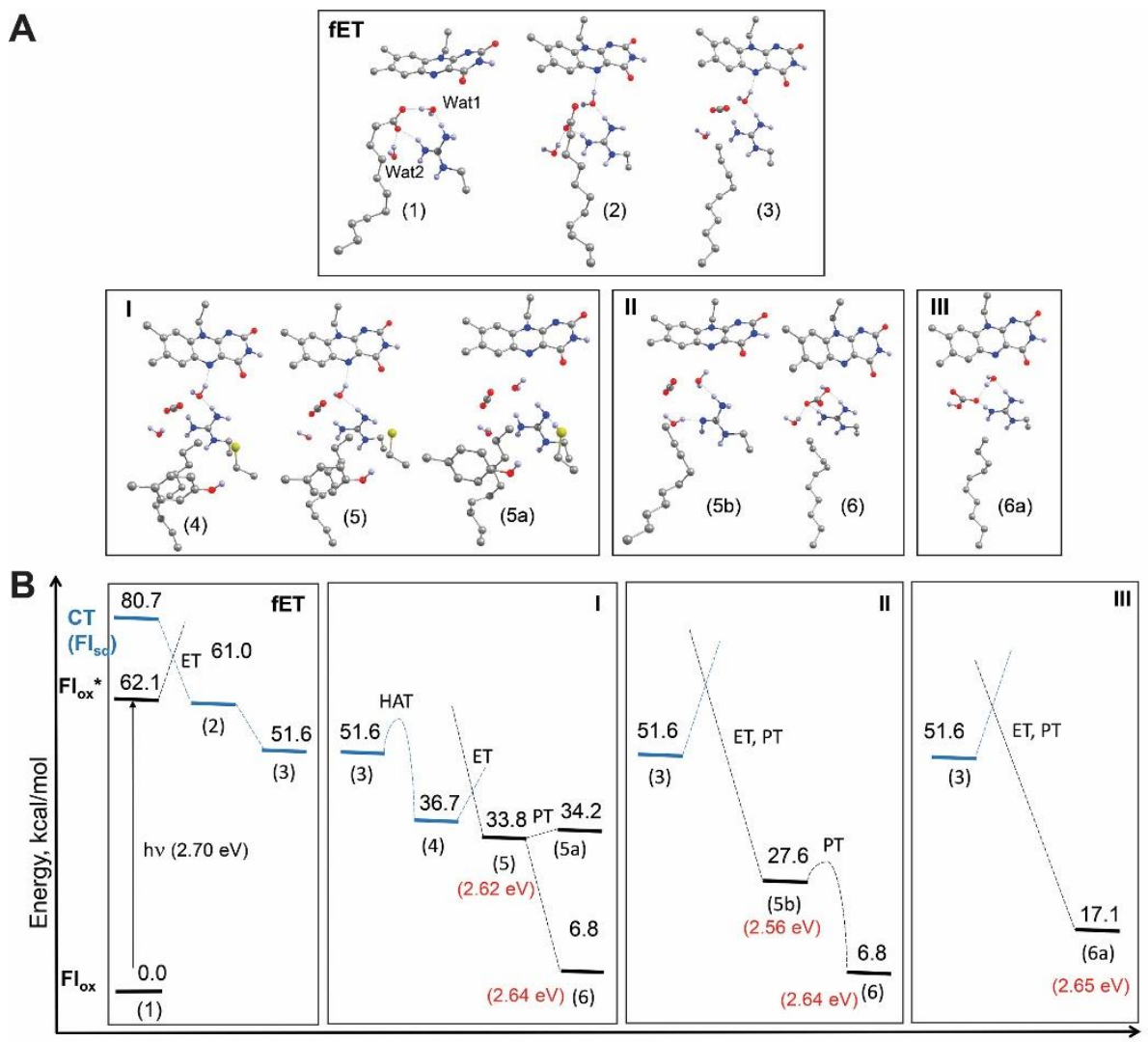

C
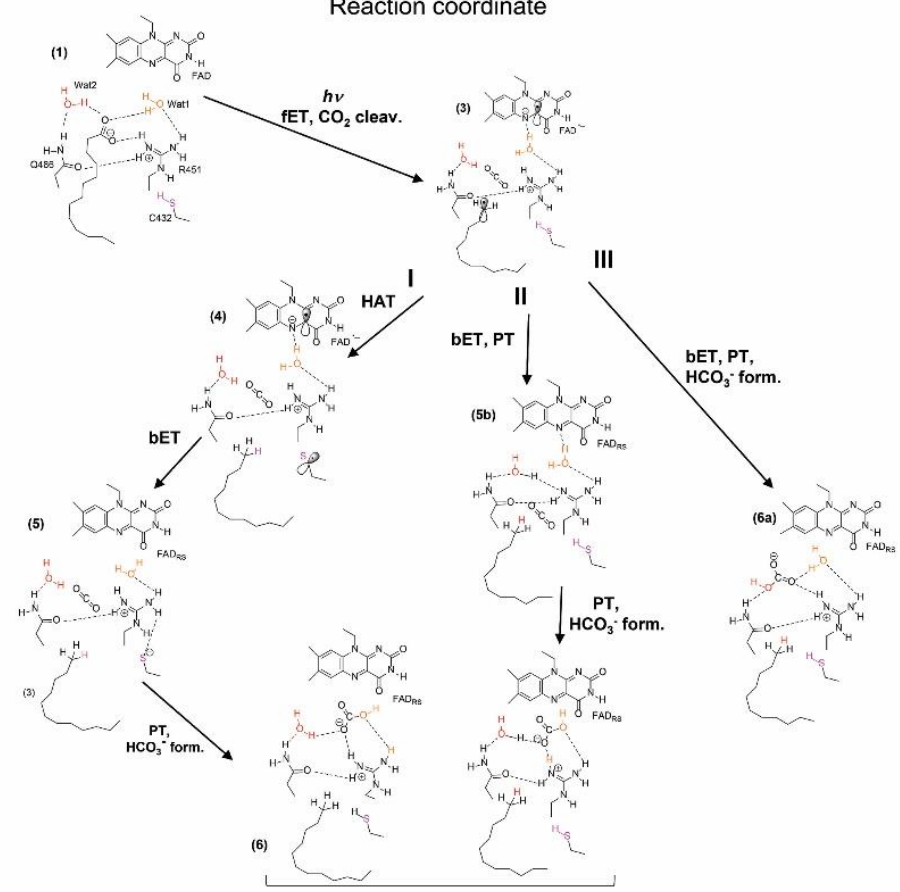
1 Fig. 6. Plausible CvFAP photoreaction pathways according to quantum-chemistry

2 calculations. (A) Structural changes accompanying decarboxylation and alkane formation in the

3 FAP active site. Flavin butterfly bending angle is $15^{\circ}$ and $19^{\circ}$ in the oxidized and semiquinone

4 states, respectively. For clarity, only a small part of the active site model is shown. The complete

5 model is presented in fig. S16. (B) Energies of the reactants, intermediates and products indicated

6 in (A). Common to all pathways is fET mediated by Wat1 rearrangement (1) $\rightarrow(2)$ followed by

$7 \quad \mathrm{CO}_{2}$ cleavage (2) $\rightarrow$ (3). Alkan formation occurs either via HAT (pathway I) or PCET (pathways

8 II and III). Pathway I consist of alkane formation via HAT from C432 (3) $\rightarrow$ (4), flavin re-oxidation

9 by the C432-radical (4) $\rightarrow(5)$, and C432 re-protonation and bicarbonate formation (5) $\rightarrow(6)$.

10 Pathway II involves alkane formation via bET coupled to proton transfer (PT) from Wat2 and

11 subsequent R451 deprotonation $(3) \rightarrow(5 b)$. Bicarbonate formation from $\mathrm{CO}_{2}$ and Wat1 recovers

12 protonated R451 (5b) $\rightarrow(6)$. In pathway III, bET is coupled to Wat 2 deprotonation and formation

13 of bicarbonate. (3) $\rightarrow(6 a)$. (C) Chemical scheme detailing intermediate states of fET and pathways

14 I-III. 

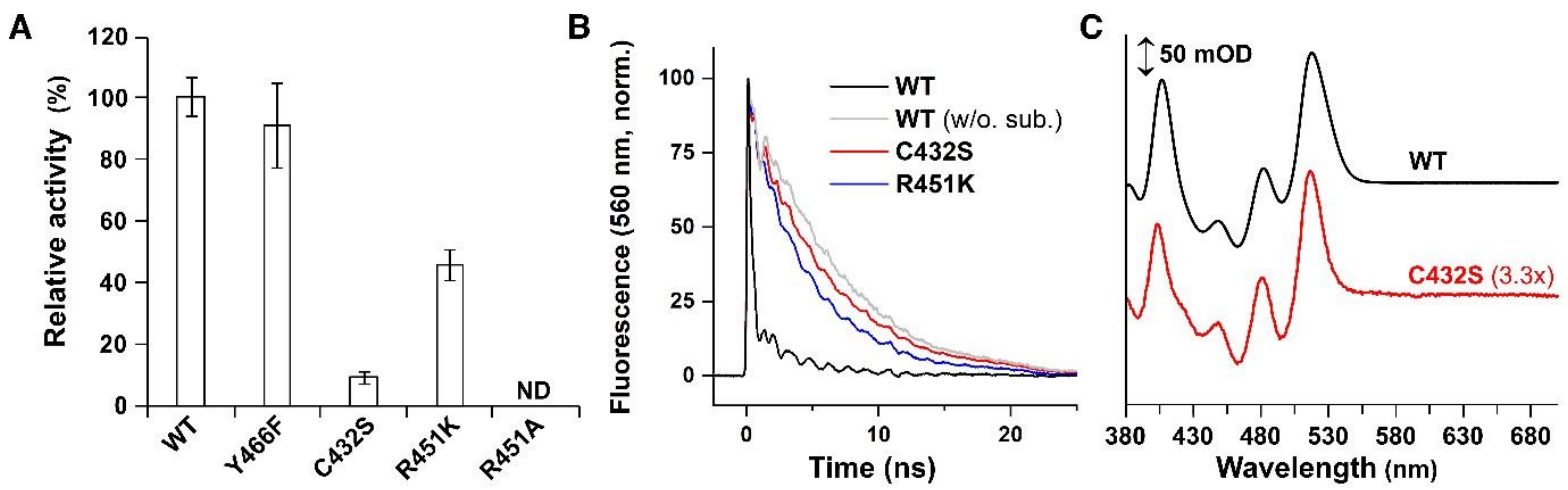

D

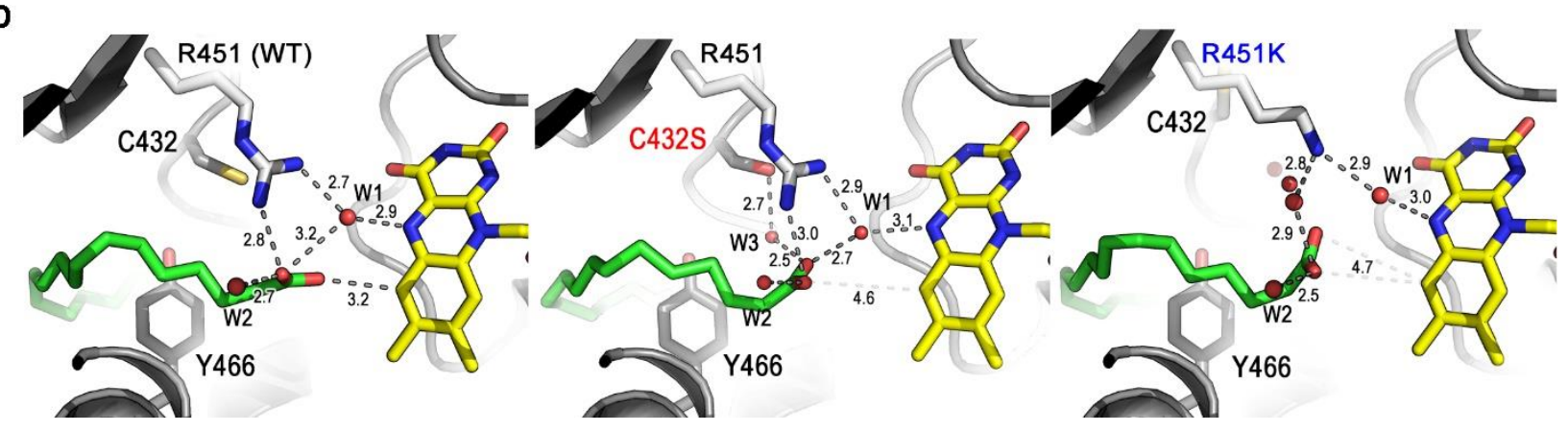

Fig. 7. Impact of mutations of conserved residues on $C v$ FAP activity, ${ }^{1}$ FAD* fluorescence

4 decay, spectrum of cryo-trapped FAD $_{\mathrm{RS}}$ and active site structure. (A) Activities of purified

5 recombinant mutant $C \nu$ FAPs relative to WT (measured by gas chromatography coupled with mass

6 spectrometry for the $\mathrm{Y} 466 \mathrm{~F}, \mathrm{R} 451 \mathrm{~K}$, and R451A mutants) in presence of cis-vaccenic acid as

7 substrate. Relative activity of the C432S mutant was measured by membrane inlet mass

8 spectrometry in order to avoid activity underestimation due to low photostability of this particular

9 mutant under continuous illumination conditions. All activities were normalized to FAD content.

10 Mean \pm SD ( $\mathrm{n}=5$ repeats). (B) Normalized time-resolved fluorescence at $560 \mathrm{~nm}$ of WT, C432S

11 and $\mathrm{R} 451 \mathrm{~K} \mathrm{C \nu FAP}$ in the presence of native substrates (and after their consumption in WT); for

12 Y466F and R451A mutants, see fig. S25B. (C) Light minus dark spectrum of WT and mutant

$13 \mathrm{C} 432 \mathrm{~S}$ at $200 \mathrm{~K}$ obtained by cryo-UV-Vis spectroscopy and normalized on FAD content. (D)

14 Structure of the active site of the WT (left), C432S (middle) and R451K (right) mutants. Distances 
1 (in $\AA$ ) between substrate (green), FAD (yellow), water molecules (red) and amino acid residues

2 (white/gray) are shown. Compared to WT, in the C432S and R451 mutants the FA carboxylate is

3 rotated $\sim 50^{\circ}$ around an axis defined by the fatty acid atoms $\mathrm{O} 2$ and $\mathrm{C} 2$.

4
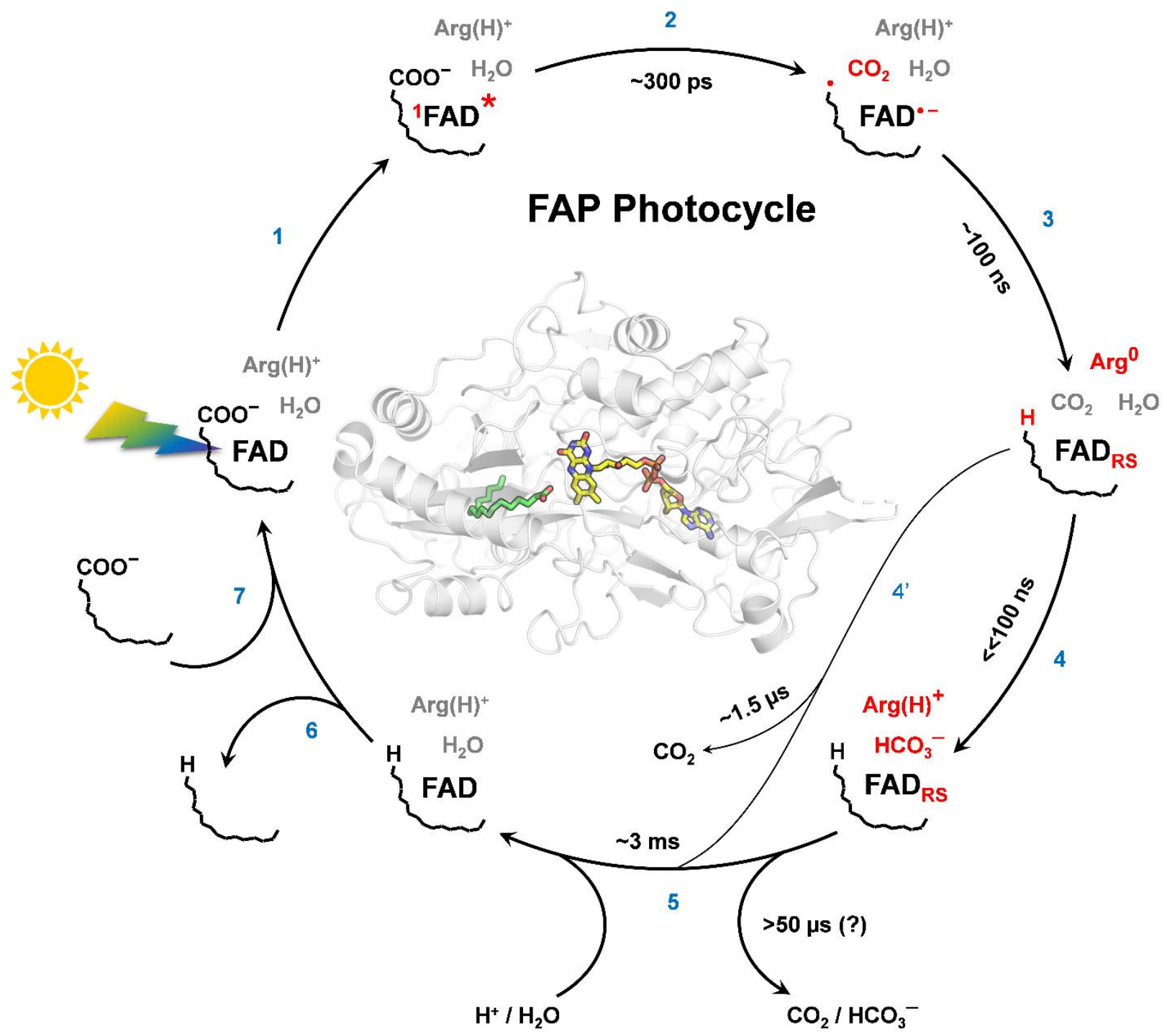
2 Fig. 8. Suggested $\boldsymbol{C} v$ FAP photocycle. Upon light excitation (1), fET in $\sim 300$ ps from the fatty

3 acid anion to ${ }^{1} \mathrm{FAD}^{*}$ (observed by ultrafast fluorescence and transient absorption spectroscopies)

4 leads to its quasi-instantaneous decarboxylation (2), as observed by TR-IR and TR-SFX and

5 supported by the computed absence of an energy barrier. bET in $\sim 100 \mathrm{~ns}$ from $\mathrm{FAD}^{\bullet-}$ (presumably

6 to the alkyl radical) results in formation of red-shifted (re-)oxidized flavin FAD $\mathrm{RS}_{\text {; }}$, the H/D KIE

7 suggests bET is coupled to and/or limited by PT. Cryotrapping FTIR experiments suggest arginine

8 as the final proton donor to the alkyl (3). Concomitantly, most $\mathrm{CO}_{2}(\sim 75 \%)$ is transformed (4) to

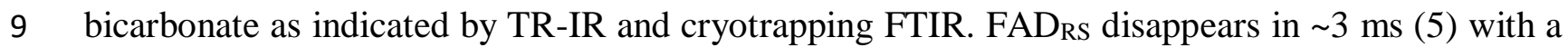

10 H/D KIE > 3, indicating coupling to PT. Upon alkane release (6), new substrate binds (7). About

$1125 \%$ of the formed $\mathrm{CO}_{2}$ is not transformed to bicarbonate, likely because it migrates away from the

12 active site within $100 \mathrm{~ns}$, leaving the protein in $\sim 1.5 \mu \mathrm{s}\left(4^{\prime}\right)$. In this minor fraction, arginine (R451)

13 should re-protonate at latest in the $\sim 3 \mathrm{~ms}$ step (5). Changes after individual steps are marked in red,

14 time constants are for room temperature. 


\section{Supplementary Materials for}

\section{Mechanism and dynamics of fatty acid photodecarboxylase}

D. Sorigué ${ }^{1}$, K. Hadjidemetriou ${ }^{2}$, S. Blangy ${ }^{1}$, G. Gotthard ${ }^{3}$, A. Bonvalet ${ }^{4}$, N. Coquelle ${ }^{5}$, P.

8 Samire $^{\mathbf{1 , 6}}$, A. Aleksandrov ${ }^{4}$, L. Antonucci ${ }^{4}$, A. Benachir ${ }^{4}$, S. Boutet ${ }^{7}$, M. Byrdin ${ }^{2}$, M. 9 Cammarata $^{8}$, S. Carbajo ${ }^{7}$, S. Cuiné ${ }^{1}$, R. B. Doak ${ }^{9}$, L. Foucar ${ }^{9}$, A. Gorel ${ }^{9}$, M. Grünbein ${ }^{9}$, E.

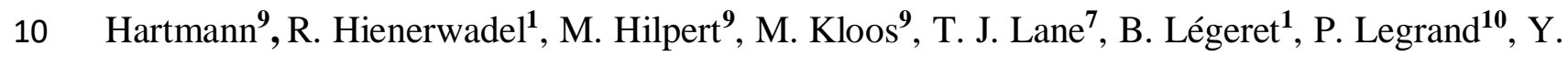
11 Li-Beisson ${ }^{1}$, S. L. Y. Moulin¹, D. Nurizzo ${ }^{3}$, G. Peltier ${ }^{1}$, G. Schirò ${ }^{2}$, R. L. Shoeman ${ }^{9}$, M. Sliwa ${ }^{11}$, 12 X. Solinas ${ }^{4}$, B. Zhuang ${ }^{4,6}$, T. R.M. Barends, J.-P. Colletier ${ }^{2}$, M. Joffre ${ }^{4}$, A. Royant ${ }^{2,3}$, C.

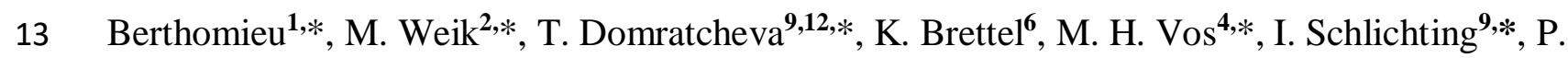

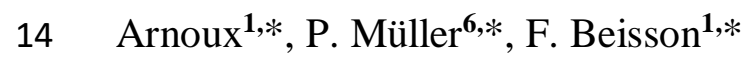

17 Correspondence to: frederic.beisson@cea.fr (F.B.), pavel.muller@i2bc.paris-saclay.fr (P.M.), pascal.arnoux@cea.fr (P.A.), Ilme.Schlichting@mpimf-heidelberg.mpg.de (I.S.), marten.vos@polytechnique.edu (M.H.V.), Tatjana.Domratcheva@ mpimf-heidelberg.mpg.de (T.D.), martin.weik@ibs.fr (M.W.), catherine.berthomieu@cea.fr (C.B.) 
This PDF file includes:

2

3

Materials and Methods

4

Supplementary Text S1 to S6

5

Figures. S1 to S31

6

Tables $\mathrm{S} 1$ to $\mathrm{S} 11$

7

8 Other Supplementary Materials for this manuscript include the following:

9

10

Copies of Full wwPDB X-ray Structure Validation Report

11

12

13

14

15

16 


\section{Table of contents}

Materials and Methods $\quad 1$

$5 \quad$ Expression and purification of recombinant CvFAP and mutant FAPs 1

6 Analysis of FAP endogenous fatty acids by LC-MS 2

$7 \quad$ FAP activity assays 3

8 FTIR spectroscopy 4

9 Protein crystallization and structure determination for static crystallography 5

10 In crystallo optical spectroscopy of single crystals 7

11 Absorbed X-ray dose calculations in in crystallo optical spectroscopy and synchrotron crystallography experiments 8

12 Time-resolved fluorescence on ps to ns timescales 9

13 Transient absorption spectroscopy on ns to ms timescales 10

14 Ultrafast visible absorption and fluorescence spectroscopy 11

15 Excitation power dependence 12

16 Multi-scale transient infrared absorption spectroscopy 12

17 Microcrystal growth and injection for TR-SFX 14

18 Picosecond pump-laser excitation of FAP microcrystals in TR-SFX experiments 15

19 TR-SFX data collection and online monitoring 16

20 SFX data processing 17

21 SFX dark structure solution and refinement 18

22 Calculation of difference Fourier maps between SFX data sets with and without laser excitation 19

23 Molecular dynamics simulations 20

24 Quantum chemistry calculations 20

25 Comparison of GMC oxidoreductase sequences 21

26 Supplementary Text 22

27 S1. Study of FAD bending by low-dose X-ray crystallography, microspectrophotometry and molecular dynamics 22

28 S2. Band assignment in FTIR spectroscopy 22

29 S3. Time-resolved fluorescence in solution on ps to ns timescales 27

30 S4. Transient absorption spectroscopy in solution on ns to ms timescales 31

31 S5. Time-resolved fluorescence spectroscopy of CvFAP crystals 36

32 S6. Quantum chemistry study of the CvFAP reaction mechanism 37

33 6.1. Model of the CvFAP active site 37

34 6.2. Forward electron transfer (fET) is facilitated by rearrangement of Wat1 38

35 6.3. Fatty acid decarboxylation in the charge-transfer (CT) state 40

36 6.4. Stabilization of the alkyl-radical intermediate in FAP 41 
6.8. Excitation and redox energies of bent flavin $\quad 45$

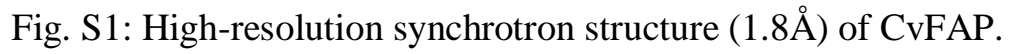

Fig. S2: Isoalloxazine ring of FAD.

Fig. S3: X-ray dose-resolved UV-Vis absorption spectra of a CvFAP crystal at room temperature.

Fig. S4: Low dose structure at room temperature and X-ray dose-resolved Raman spectra series of a CvFAP crystal at $100 \mathrm{~K}$.

Fig. S5: Flavin dihedral angle observed in Molecular Dynamics simulations.

Fig. S6: Residual $\mathrm{mF}_{\mathrm{obs}}-\mathrm{DF}_{\text {calc }}$ peaks indicate isoalloxazine rings of the FAD cofactor are not planar in the SFX darkstate structure.

Fig. S7: FAD bending in the SFX dark-state structure.

Fig. S8: Ultrafast fluorescence and visible absorption spectroscopy on FAP in solution in the presence of substrate. 56

Fig. S9: Formation of $\mathrm{CO}_{2}$ co-product studied by TR-IR.

Fig. S10: Effects of $\mathrm{pH}$ and substrate consumption on the transient FAD absorption changes in WT CvFAP.

Fig. S11: Summary of the results obtained on CvFAP using time-resolved infrared and UV-Visible spectroscopies. 59

Fig. S12: Absorption spectra of the red-shifted intermediate in CvFAP crystals and solution.

Fig. S13: Characterization of the red-shifted intermediate in CvFAP crystals.

Fig. S14: Environment of the bicarbonate modelled in the electron density next to $\mathrm{C} 432$ in the $150 \mathrm{~K} \mathrm{pH} 8.5$ structure ('150 K light').

Fig. S15: Fluorescence decays measured using single photon counting technique.

Fig. S16: Model of the CvFAP active site.

Fig. S17: Fatty acid decarboxylation in the charge-transfer (radical) state.

Fig. S18: Energy and transition state of HAT reaction.

Fig. S19: Bicarbonate formation from $\mathrm{CO}_{2}$ and Wat1.

Fig. S20: Suggested red-shifted $F_{A D}$ intermediates.

Fig. S21: Flavin butterfly bending energies.

Fig. S22: Interaction of the fatty acid carboxylate with water molecules in wild type and C432S models. 70

Fig. S23: Conservation of residues specific to FAPs within the active site of the GMC oxidoreductases.

Fig. S26: Comparison of the light-induced FTIR difference spectra of CvFAP and CvFAP mutants recorded at $100 \mathrm{~K}$, and omit map electron density maps of R451K, R451A and C432S mutants.

Fig. S27: Analysis of the fatty acids associated with purified WT CvFAP. 
1 Fig. S29: Filtering effect on FAP microcrystal size.

2 Fig. S30: Depth-dependent photon absorption regimes inside FAP crystals

8 Table S4: list of sequences of FAPs and other GMC oxidoreductases used for protein alignment and sequence logo. 88

9 Table S5: R-/U-B3LYP energies and energy gradient of the optimized geometries modelling alkane production by the 10 FAP enzyme.

11 Table S6: Excitation energy calculations for geometries (1) and (2).

12 Table S7: Electronic couplings for states involved in fET and bET.

Table S8: Excitation energy calculations for suggested oxid. flavin red-shifted intermediates (5), (5b), (6) and (6a). 93 
1 Materials and Methods

2 Expression and purification of recombinant $C \nu$ FAP and mutant FAPs

3 The FAP used in all experiments is the previously published recombinant $C v \mathrm{FAP}$ (4), from

4 Chlorella variabilis NC64A ( $C v$ FAP or single mutant of it) with an N-terminal truncation of 14

5 amino residues. This $C \nu \mathrm{FAP}$ corresponds to residues 76-654 of the full-length amino acid

6 sequence. Site-directed $C v$ FAP mutants C432A, C432S, R451A, R451K and Y466F were

7 obtained by introducing point mutations in the previously reported FAP synthetic gene codon-

8 optimized for E. coli expression (4). The primers used to generate the mutants and the truncated

9 version of the $C \nu \mathrm{FAP}$ are listed in Table S3; for the C432S mutant, a part of the gene (between

10 the MfeI and EcoRI restriction sites) was synthesized (Eurofins Genomics Germany GmbH),

11 thereby introducing the mutation, and then exchanged. Coding sequences for $C v \mathrm{FAP}$ and its

12 mutants were cloned into a pET-28 based expression vector containing his-tagged thioredoxin

13 downstream the gene and recombinant proteins were expressed. The recombinant FAP and

14 mutant proteins were produced in BL21-CodonPlus (DE3)-RIL E. coli cells (BL21-Rosetta

15 (DE3) E. coli for the $\mathrm{C} 432 \mathrm{~S}$ mutant) cultured in $\mathrm{TB}$ medium at $37^{\circ} \mathrm{C}$ up to OD 1 . At this stage,

16 cultures were induced with $500 \mu \mathrm{M}$ IPTG and the temperature was decreased to $17^{\circ} \mathrm{C}$. Cells

17 were grown for an additional $24 \mathrm{~h}$. The cells were harvested by centrifugation at $4000 \mathrm{~g}$ for 30

$18 \mathrm{~min}$ and the pellet was frozen. Purification of recombinant protein was performed as previously

19 described (4). Briefly, cell pellet was resuspended in lysis buffer containing $300 \mathrm{mM} \mathrm{NaCl}, 50$

$20 \mathrm{mM}$ Tris $\mathrm{pH}$ 8.0, $10 \mathrm{mM}$ imidazole, 5\% (w/v) glycerol, $0.25 \mathrm{mg} \mathrm{mL}^{-1}$ lysozyme, $20 \mathrm{mM} \mathrm{MgSO}_{4}$,

$2110 \mu \mathrm{g} \mathrm{mL}^{-1}$ DNase, and antiproteases (SIGMAFAST ${ }^{\mathrm{TM}}$ Protease Inhibitor Tablets). After

22 resuspension, cells were lysed by sonication (sonicator probe at a frequency of $20 \mathrm{kHz}$, four 45 -

23 second cycles separated by a one-minute delay) and centrifuged during $30 \mathrm{~min}$ at $12,000 \mathrm{~g}$. The 
1 supernatant was collected and the enzyme was purified by FPLC. The first purification step was

2 made using a nickel column. Tobacco etch virus (TEV) protease (at $1 \mathrm{mg}$ per $20 \mathrm{mg}$ total protein)

3 was used to cut off the His tag and the thioredoxin. A dialysis was performed overnight in the

4 presence of TEV protease to change the buffer to $300 \mathrm{mM} \mathrm{NaCl}, 50 \mathrm{mM}$ Tris $\mathrm{pH} 8.0,10 \mathrm{mM}$

5 imidazole, 5\% (w/v) glycerol. A second FPLC chromatography step using a nickel column was

6 made to separate the protein from the His-tagged thioredoxin. The last purification step made

7 use of a gel filtration column HiLoad Superdex $200(26 / 600 \mathrm{~mm})$. Buffer used for this step

8 contained $150 \mathrm{mM} \mathrm{NaCl}, 10 \mathrm{mM}$ Tris $\mathrm{pH} 8.0,5 \%(\mathrm{w} / \mathrm{v})$ glycerol. The protein was concentrated

9 using Amicon ${ }^{\circledR}$ Ultra Centrifugal filters (30 kDa), flash-cooled in liquid nitrogen and stored at

$10-80^{\circ} \mathrm{C}$. Protein concentration was calculated using absorbance at $280 \mathrm{~nm}$ taking into account the

11 contribution of FAD. Almost $60 \%$ of endogenous fatty acids associated with purified FAP were

12 C18 fatty acids (fig. S27).

\section{Analysis of FAP endogenous fatty acids by LC-MS}

15 Briefly, $50 \mu \mathrm{L}$ of the purified WT FAP $\left(6 \mathrm{mg} \mathrm{mL}^{-1}\right.$ in $150 \mathrm{mM} \mathrm{NaCl}, 10 \mathrm{mM}$ Tris, $\mathrm{pH} 8.0,5 \%$

$16(\mathrm{w} / \mathrm{v})$ glycerol buffer) was heated at $85^{\circ} \mathrm{C}$ for $10 \mathrm{~min}$ with $50 \mu \mathrm{L}$ of ethanol/acid acetic $(0.1 \%$

$17 \mathrm{v} / \mathrm{v})$ and centrifuged at $14,500 \mathrm{~g}$ for $10 \mathrm{~min}$. The supernatant $(2 \mu \mathrm{L})$ was analyzed by

18 UPLC/MS/MS using a Kinetex column (C18 2.1 × $150 \mathrm{~mm} 1.7 \mu \mathrm{m}$, Phenomenex) mounted on

19 an ultimate RS 3000 UPLC system (Thermo Fisher). A binary gradient system of

20 acetonitrile-water $(60: 40, \mathrm{v} / \mathrm{v})$ and isopropanol-acetonitrile $(90: 10, \mathrm{v} / \mathrm{v})$, both containing 10

$21 \mathrm{mM}$ ammonium formate, was used as eluent $\mathrm{A}$ and $\mathrm{B}$, respectively. The elution was performed

22 with a gradient of $30 \mathrm{~min}$; eluant B was increased from 7 to $97 \%$ in 26 min and held constant for

235 min, solvent B was then decreased to $7 \%$ and held constant for another 6.9 min for column re- 
1 equilibration. The flow-rate was $0.3 \mathrm{~mL} / \mathrm{min}$. The column oven temperature was kept at $45^{\circ} \mathrm{C}$.

2 This LC system is connected to a quadrupole-time-of-flight (QTOF) 5600 mass spectrometer

3 (AB Sciex, Framingham, MA, USA) equipped with a duo-spray ion source operating in negative 4 mode (4500V). Identification of fatty acids was based on mass accuracy peaks from the MS

5 scan. The percentage of each fatty acid in total fatty acids was calculated with multiquant

6 software (AB Sciex) on the basis of intensity values of extracted masses of identified fatty acids.

\section{$8 \quad$ FAP activity assays}

9 FAP activities were measured by gas chromatography coupled to mass spectrometry (GC-MS) 10 for WT and mutants Y466F, R451K, R451A and C432S. In the case of mutants with low

11 (C432S) or no activity detected by GC-MS (R451A), the activity was also measured by 12 membrane inlet mass spectroscopy (MIMS) using WT as a reference. For activity measurements 13 performed by GC-MS, enzymatic assays, extraction and quantification of hydrocarbons were 14 performed as previously described (4). For activity measurements based on MIMS, $20 \mu \mathrm{L}$ of 15 purified enzyme (WT, C432S or R451K mutant) at $30 \mu \mathrm{M}$ concentration were added to a reaction 16 medium composed of $1.45 \mathrm{~mL}$ of Tris/Citrate/Borate buffer (100 mM, pH 6.5) containing $\mathrm{NaCl}$ $17100 \mathrm{mM}$ and supplemented with $50 \mu \mathrm{L}$ of hexadecanoic acid-1 $-{ }^{13} \mathrm{C}(10 \mathrm{mM}$ in 18 dimethylsulfoxide) and 750 units of bovine carbonic anhydrase (Sigma Aldrich C3934). The 19 measuring chamber was thermo-regulated at $25^{\circ} \mathrm{C}$, and stirred continuously and illuminated with 20 continuous $460 \mathrm{~nm}$-emitting diode (Luminus PT39-B-L21). The ${ }^{12} \mathrm{CO}_{2}(\mathrm{~m} / \mathrm{z}=44)$ and ${ }^{13} \mathrm{CO}_{2}$ $21(\mathrm{~m} / \mathrm{z}=45)$ released by FAP were monitored as described in $(41)$. 
2 For experiments involving exchange of the substrates present in the FAP, $50 \mu \mathrm{L}$ of protein at $3135 \mathrm{mg} \mathrm{mL}^{-1}$ was diluted in $2 \mathrm{~mL}$ of buffer $(150 \mathrm{mM} \mathrm{NaCl}, 10 \mathrm{mM}$ Tris $\mathrm{pH} 8.0)$. Ten $\mu \mathrm{L}$ of 4 purified palmitate or $1-{ }^{13} \mathrm{C}$-palmitate (stock solution $100 \mathrm{mM}$ in ethanol or DMSO) was added 5 and the samples were illuminated for $10 \mathrm{~s}$ with white light at an intensity of $8000 \mu \mathrm{moles} \mathrm{m}^{-2} \mathrm{~s}^{-}$

61 . In order to remove excess substrate and proteins that could have precipitated during the 7 incubation and illumination steps, the samples were then centrifuged $45 \mathrm{~min}$ at 20,000g. After

8 centrifugation, the supernatant was recovered and concentrated using Amicon® Ultra 9 Centrifugal Filters $(30 \mathrm{kDa})$. In order to eliminate ethanol or DMSO, the dilution and 10 concentration steps were repeated twice. The final FAP concentration was adjusted to $135 \mathrm{mg}$ $11 \mathrm{~mL}^{-1}$.

12 For experiments with fully labeled ${ }^{15} \mathrm{~N}-\mathrm{FAP}$, the recombinant FAP protein was produced 13 in cells cultured in M9 minimal medium supplemented with ${ }^{15} \mathrm{NH}_{4} \mathrm{Cl}$ as sole nitrogen source and 14 purified as described above. For experiments in $\mathrm{D}_{2} \mathrm{O}$ (Fig. 3A), the buffer $(10 \mathrm{mM}$ Tris- $\mathrm{HCl} \mathrm{pH}$ 158.0 with $150 \mathrm{mM} \mathrm{NaCl}$ ) was lyophilized to dry powder and the sublimated $\mathrm{H}_{2} \mathrm{O}$ was replaced by $16 \mathrm{D}_{2} \mathrm{O}$, up to the original volume of the $\mathrm{H}_{2} \mathrm{O}$ buffer. For sample preparation, $50 \mu \mathrm{L}$ of purified 17 FAP at a $135 \mathrm{mg} \mathrm{mL}^{-1}$ concentration was diluted in $1 \mathrm{~mL}$ of the $\mathrm{D}_{2} \mathrm{O}$ buffer. The sample was 18 then concentrated using Amicon® Ultra Centrifugal Filters (30 kDa). These dilution and 19 concentration steps were carried out twice to optimize the replacement of the exchangeable 20 protons by deuterium. The final concentration of the sample was adjusted to $135 \mathrm{mg} \mathrm{mL}^{-1}$.

21 Except for exchanging substrate, all sample preparation steps were performed in the dark 22 or under non-actinic light conditions $(>550 \mathrm{~nm})$ to prevent enzyme activation. For FTIR 23 experiments, $4 \mu \mathrm{L}$ of the protein solution was deposited between two $\mathrm{CaF}_{2}$ windows and the 
1 sample absorption was adjusted below 0.9 absorption units for the absorption of the main band

2 at $1640 \mathrm{~cm}^{-1}$ (corresponding to the Amide I and water bending modes).

FTIR spectra were recorded with an IFS 66 FTIR spectrometer (Bruker) equipped with

4 a MCT-A detector and a KBr beam-splitter. Experiments at 100 or $150 \mathrm{~K}$ were performed using

5 a TBT $\mathrm{N}_{2}$-flow cryostat equipped with quartz windows for transmission in the UV-Vis range

6 and with a combination of CVD-Diamond and ZnSe windows for IR transmission. Illumination

7 was achieved with a continuous $460 \mathrm{~nm}$-emitting diode (Luminus PT39-B-L21). Light - minus -

8 Dark difference spectra were obtained by subtraction of spectra recorded under illumination

9 (Light) and before illumination (Dark). Scans (300 to 400 for each spectrum) were recorded at a

$104 \mathrm{~cm}^{-1}$ resolution (acquisition time was between 1.5 and 2 minutes). Depending on the samples,

11 spectra were recorded with one sample or with up to six samples (R451K mutant) and averaged

12 to increase the signal-to-noise ratio. Similar light-induced experiments were performed in the

$13 \mathrm{UV}$-Vis domain at 100 or $200 \mathrm{~K}$ using a Cary 100 spectrophotometer equipped with the TBT

14 cryostat. Spectra were measured between 380 and $600 \mathrm{~nm}$ with $1 \mathrm{~nm}$ steps, using custom-made

15 polyethylene cuvettes with a pathlength of $1 \mathrm{~mm}$. Glycerol at a 70 to $85 \%(\mathrm{v} / \mathrm{v})$ concentration

16 was added to the concentrated FAP sample (final FAD concentration around $200 \mu \mathrm{M}$ ). Spectra

17 were normalized based on FAD content.

19 Protein crystallization and structure determination for static crystallography

20 FAP $\left(C \nu\right.$ FAP and mutant R451A, R451K and C432S) were concentrated to around $6 \mathrm{mg} \mathrm{mL}^{-1}$

$21(97 \mu \mathrm{M})$ in $150 \mathrm{mM} \mathrm{NaCl}, 10 \mathrm{mM}$ Tris $\mathrm{pH}$ 8.0, 5\% (w/v) glycerol. Crystallization screening was

22 performed using 96-wells plates in a sitting drop setup using a Freedom EVO 100 pipetting robot

23 (Tecan). Screening was done by mixing $0.7 \mu \mathrm{L}$ of protein at $6 \mathrm{mg} \mathrm{mL}^{-1}$ with $0.7 \mu \mathrm{L}$ of a mother 
1 liquor from various commercial screens. Hits were obtained in position H8 of the Stura Footprint

2 Combination screen (20\% (w/v) PEG 4000, $20 \%$ (w/v) 2-propanol, 0.1M Na-Citrate pH 5.5) at

$3 \quad 8^{\circ} \mathrm{C}$ in the dark. Therefore, removing the $\mathrm{N}$-terminal part of the protein did not change drastically

4 crystallization conditions compared to the longer $C v$ FAP construct previously used (4). The final

5 crystallization buffer was 25-40\% (w/v) PEG 4000, $100 \mathrm{mM} \mathrm{Na-Citrate} \mathrm{pH} \mathrm{5.5,} 10 \mathrm{mM}$

6 spermidine. After mixing $0.7 \mu \mathrm{L}$ of protein with $0.7 \mu \mathrm{L}$ of crystallization buffer, crystallization

7 plates were stored at $8^{\circ} \mathrm{C}$, and diamond-shaped crystals appeared after three days and took about

8 one week to reach their final size (around $100 \times 150 \times 100 \mu \mathrm{m}^{3}$ ). Mutants R451A, R451K and

9 C432S crystallized under the same conditions. Visualization and manipulation of $C v F A P$ and

10 mutant crystals were done in the dark using a microscope with a red filter eliminating

11 wavelengths below $600 \mathrm{~nm}$. For cryoprotection, crystals were transferred in a drop of mother

12 liquor (40\% (w/v) PEG 4000, $100 \mathrm{mM}$ Na citrate) supplemented with 5\% (w/v) glycerol before

13 flash cooling directly in liquid nitrogen. Diffraction data were collected at the ESRF (Grenoble)

14 on beamlines BM30A-FIP (42), ID29 (43) and ID30A-3 (44), at the Soleil PX1 beamline (Saint-

15 Aubin, France) and PXII at the Swiss Light Source. Crystals of CvFAP, R451K and C432S

16 belong to space group $I 222$ and diffraction data were recorded respectively up to 1.8 or $2 \AA$

17 resolution. Crystals of the R451A variant belong to space group $P 2{ }_{1} 2_{1} 2_{1}$ and diffract X-rays to

$182 \AA$ A resolution. A high-resolution structure ' $100 \mathrm{~K}$ dark' was solved by molecular replacement

19 using the medium-resolution structure (4) as a starting model, and other structures (referred to

20 as ' $100 \mathrm{~K}$ light', ' $150 \mathrm{~K}$ light', 'RT dark LD', 'R451K' and 'R451 A) using the $100 \mathrm{~K}$ dark with

21 the Molrep program from the ccp4 suite (45). The ' $100 \mathrm{~K}$ light' structure was obtained after

22 illuminating a crystal (grown at pH5.5) at $100 \mathrm{~K}$, using a $5 \mathrm{~mW} 470 \mathrm{~nm}$ LED (see fig. S12 for

23 spectra). The ' $150 \mathrm{~K}$ light' structure was obtained after soaking a crystal in a mother liquor in 
1 which the citrate buffer was replaced by $100 \mathrm{mM}$ Tris $\mathrm{pH} 8.5$ for 10 minutes. Crystal was then

2 flash frozen directly in liquid nitrogen and then placed in a stream of gaseous nitrogen set at 150

$3 \mathrm{~K}$. This crystal was illuminated for 30 minutes to promote the formation of $F A D_{R S}$ state before

4 data collection. All structures were refined using Refmac (46) with manual building done using

5 the Coot program (47).

6

\section{$7 \quad$ In crystallo optical spectroscopy of single crystals}

8 icOS experiments were performed on $100 \times 100 \times 100 \mu \mathrm{m}^{3}$ WT FAP crystals at room or

9 cryogenic temperature. In the latter case, the crystal was either cryoprotected with glycerol or

10 MPD (2-Methyl-2,4-pentanediol). The appearance of the FAP $\mathrm{RS}_{\mathrm{RS}}$ state was monitored at $100 \mathrm{~K}$

11 by following the evolution of the UV-Vis absorption spectrum of a FAP crystal under irradiation

12 by a $470 \mathrm{~nm}$ LED at the icOS Lab (44). Similarly, this method was used to determine the dose

13 resulting in a reduction of the FAD by following the evolution of the absorption spectrum from

$14 \mathrm{FAD}_{\mathrm{OX}}$ to $\mathrm{FAD}_{\mathrm{RS}}$. The half-dose of X-ray photoreduction of FAP crystals was determined to be

$15 \sim 40 \mathrm{kGy}$ at room temperature (fig. S3) on beamline BM30A-FIP (42) using online UV-Vis

16 absorption microspectrophotometry (48). Online Raman spectroscopy to monitor X-ray induced

17 damage to the FAD was performed on a FAP crystal at $100 \mathrm{~K}$ on beamline ID29 (43), as

18 previously described (49). Most of the peaks hardly show any decrease in their intensity (fig.

19 S4B,C), in contrast with an online Raman study showing an X-ray-induced butterfly bending of

20 a protein-encased flavin that comes with reduction and is accompanied by a decrease of Raman

21 bands intensities (26). Only five stretching modes are affected upon progressive irradiation (4

22 decreases, 1 increase) and are thus characteristic of the change in electronic structure of the FAD. 
1 Absorbed X-ray dose calculations in in crystallo optical spectroscopy and synchrotron

2 crystallography experiments

3 Average absorbed doses were calculated using RADDOSE-3D (http://raddo.se/) (50) using data

4 collection parameters and characteristics of the used beamlines. All crystals were of $100 \times 100$

$5 \times 100 \mu \mathrm{m}^{3}$ dimensions. The low dose room temperature crystallography experiment was

6 performed on beamline ID30A-3 (51) on a single WT crystal using an HC1 humidity controller

7 with a humidity level calculated from the composition of the mother liquor (52). The X-ray beam

8 of ID30A-3 (fixed energy of $12.81 \mathrm{keV}$ ) has a Gaussian profile of $18 \mu \mathrm{m}$ (horizontal) $\times 14 \mu \mathrm{m}$

9 (vertical) dimensions (measured at FWHM). The X-ray flux was attenuated to $4.8 \times 10^{10} \mathrm{ph} \mathrm{s}^{-1}$,

10 and a $110^{\circ}$ oscillation wedge of diffraction data was collected in $11 \mathrm{~s}$, which amounts to an

11 average absorbed dose of $38 \mathrm{kGy}$ for the irradiated part of the crystal. The high resolution

12 cryogenic data collection of $C \nu$ FAP was performed on beamline ID29 (43) on a single crystal

13 cryocooled at $100 \mathrm{~K}$. The X-ray beam of ID29 (energy set to $12.73 \mathrm{keV}$ ) has a Gaussian profile

14 of $40 \mu \mathrm{m}$ (horizontal) $\times 30 \mu \mathrm{m}$ (vertical) dimensions (measured at FWHM) and is collimated

15 with a $50 \mu \mathrm{m}$ aperture. The X-ray flux was attenuated to $4.1 \times 10^{10} \mathrm{ph} \mathrm{s}^{-1}$, and a $127^{\circ}$ oscillation

16 wedge of diffraction data was collected in $47 \mathrm{~s}$, which amounts to an average absorbed dose of

$17154 \mathrm{kGy}$ for the irradiated part of the crystal. The online Raman microspectrophotometry

18 experiment was performed at cryogenic temperature on beamline ID29 at an energy of 11.56

$19 \mathrm{keV}$ with an X-ray flux of $6.8 \times 10^{10} \mathrm{ph} \mathrm{s}^{-1}$ on a crystal irradiated for 0 to $240 \mathrm{~s}$. The UV-Vis

20 absorption microspectrophotometry experiment was performed at room temperature using the 
$1 \mathrm{HC1}$ humidity controller on beamline BM30A-FIP (42) at an energy of $12.66 \mathrm{keV}$ with a $300 \times$

$2300 \mu \mathrm{m}^{2}$ top-hat beam with a flux of $4.1 \times 10^{10} \mathrm{ph} \mathrm{s}^{-1}$ on a crystal irradiated from 0 to $1280 \mathrm{~s}$.

\section{Time-resolved fluorescence on ps to ns timescales}

4 Fluorescence kinetics (in Figs. 3A and 7B, and fig. S25A,B) were monitored on a set-up

5 described previously $(4,53)$, using a Nd:YAG laser as the excitation light source (Continuum

6 Leopard SS-10, pulse duration of $100 \mathrm{ps}, 355 \mathrm{~nm}$, energy attenuated to $\sim 25 \mu \mathrm{J} \mathrm{cm}^{-2}$, repetition

7 rate $2 \mathrm{~Hz})$. Strong $(\sim 5 \mathrm{~mJ})$ or saturating $(\sim 10 \mathrm{~mJ})$ pre-flashes at $470 \mathrm{~nm}$ of $5 \mathrm{~ns}$ duration were

8 used to consume the native substrate when required; (Fig 7B, fig. S25A,B) were delivered by a

9 Nd:YAG-pumped optical parametric oscillator (Brillant B/Rainbow, Quantel, France). The

10 detection system consisted of a Hamamatsu microchannel plate photomultiplier tube R2566U-

11 11P (under $3.6 \mathrm{kV}$ ) connected to a digital oscilloscope (Infiniium 81004B from Agilent,

12 bandwidth DC-10 GHz, sampling rate $40 \mathrm{GSa} / \mathrm{s})$. The samples were contained in a $2 \times 2 \times 10 \mathrm{~mm}$

13 cell with self-masking solid black walls and four clear windows (Starna). Excitation pulses

14 entered through the $2 \times 10 \mathrm{~mm}$ window and fluorescence was detected in front of the $2 \times 2 \mathrm{~mm}$

15 window). An OG-530 Schott orange glass optical filter and a $560 \mathrm{~nm}$ interference filter (with a

$1610 \mathrm{~nm}$ bandwidth) were placed between the sample and the detector.

Fluorescence traces shown in Figs. 3A and 7B and fig. S25A,B are mostly averages of

1864 signals (or only 16 in the case of the signals of WT after saturating pre-flashes in fig. S25A).

$19 \sim 45 \mu \mathrm{M}$ WT and $40-70 \mu \mathrm{M}$ mutant $C \nu \mathrm{FAP}$ proteins were used in the presence of the native

20 substrate(s). The signals WT w/o substrate (Fig. 7B and fig. S25A,B) were recorded posterior to

2110 strong $(\sim 5 \mathrm{~mJ})$ pre-flashes at $470 \mathrm{~nm}$, which have consumed essentially all the bound fatty

22 acids in the excited/probed volume (see fig. S25A: the lack of significant further slowing of the 
1 fluorescence decay after 3 saturating ( $10 \mathrm{~mJ})$ pre-flashes as compared to 2 pre-flashes indicates

2 that already after the 2nd pre-flash less than $\sim 10 \%$ of the excited flavins were quenched by a

3 substrate; 10 strong $(\sim 5 \mathrm{~mJ})$ pre-flashes have consumed virtually all the native substrates).

\section{Transient absorption spectroscopy on ns to ms timescales}

5 Transient absorption kinetics were recorded on a set-up described previously $(54,55)$. The FAD

6 cofactor in FAP was excited at $470 \mathrm{~nm}$ by laser flashes of $5 \mathrm{~ns}$ duration and an energy in the

7 order of $4 \mathrm{~mJ} \mathrm{~cm} \mathrm{~cm}^{-2}$, delivered by a Nd:YAG-pumped optical parametric oscillator (Brillant

8 B/Rainbow, Quantel, France). The monitoring light was provided by three continuous wave

9 lasers: $376 \mathrm{~nm}$ - collimated diode laser Toptica iBeam smart 375-S (up to $120 \mathrm{~mW}$ ); $488 \mathrm{~nm}$ -

10 DPSS laser Picarro Cyan-20 from Spectra-Physics $(20 \mathrm{~mW}) ; 515 \mathrm{~nm}$ - DPSS laser Cobolt

11 FandangoTM $(150 \mathrm{~mW})$. The sample cell was identical to that used in the time-resolved

12 fluorescence experiments $(2 \times 2 \times 10 \mathrm{~mm})$. The monitoring light was attenuated by neutral density

13 filters and mechanically chopped to produce a rectangular pulse of $140 \mu$ s duration and an energy

14 in the order of $1 \mu \mathrm{J}$ at the entrance of the cell ( $2 \times 2 \mathrm{~mm}$ window), thus avoiding significant actinic

15 effects. This pulse was synchronized with the excitation laser flash entering the sample through

16 the $2 \times 10 \mathrm{~mm}$ window. The signals were recorded using an Alphalas UPD-500-UP photodiode

17 (rise time $<500 \mathrm{ps}$; sensitive area $0.5 \mathrm{~mm}^{2}$ ) connected via a Femto HCA electronic signal 18 amplifier (DC-325 MHz, $28 \mathrm{~dB}$ ) to a Tektronix DSA602A digital oscilloscope with bandwidth 19 limit set to $100 \mathrm{MHz}$.

For experiments on the ms timescale, the chopper was removed, the monitoring light was

21 strongly attenuated by additional neutral density filters (to 2\% intensity) and a Tektronix

22 AM502 amplifier with an upper bandwidth limit set to $100 \mathrm{kHz}$ was used. 
1 Transient absorption kinetics were measured on $C \nu \mathrm{FAP}$ proteins $(\sim 45 \mu \mathrm{M}$ WT, $40-70 \mu \mathrm{M}$

2 mutants) containing their native substrates (see Fig. 1). Addition of $\sim 500 \mu \mathrm{M}$ palmitic, vaccenic

3 or oleic acid in control experiments did not affect the kinetics, nor the signal amplitudes, only

4 the signal-to-noise ratio worsened (fatty acids are poorly soluble in water and their addition

5 causes scattering of the monitoring light). The state of the samples was controlled by UV-Vis

6 spectroscopy (Uvikon XS spectrophotometer from Secomam) prior to each experiment. All

7 signals shown in Fig. 3B,C and in figs. S10 and S25C-F were recorded upon single flash 8 excitation.

$9 \quad$ For time-resolved spectroscopic experiments in $\mathrm{D}_{2} \mathrm{O}$ (Fig. 3), our standard buffer (100 $10 \mathrm{mM}$ Tris- $\mathrm{HCl}$ buffer at $\mathrm{pH} 8.2$ with $150 \mathrm{mM} \mathrm{NaCl}$ ) was lyophilized to dry powder and the 11 sublimated $\mathrm{H}_{2} \mathrm{O}$ was replaced by $\mathrm{D}_{2} \mathrm{O}$, adding up to the original volume of the $\mathrm{H}_{2} \mathrm{O}$ buffer.

12 Isolated FAP samples in the $\mathrm{H}_{2} \mathrm{O}$ buffer were passed twice over size-exclusion columns 13 (MicroBio-Spin, Bio-Gel P-6), pre-washed and saturated with the $\mathrm{D}_{2} \mathrm{O}$ buffer. The same method 14 of buffer exchange was used to obtain FAP samples at other than 'standard' $\mathrm{pH}$ values (fig. $15 \mathrm{~S} 10 \mathrm{~A}, \mathrm{~B})$.

\section{Ultrafast visible absorption and fluorescence spectroscopy}

18 Ultrafast fluorescence and absorption spectroscopy with full spectral resolution and configured 19 for flavoproteins was performed as described (56), with a setup operating at $500 \mathrm{~Hz}$. The $\sim 100$

$20 \mathrm{fs}, \sim 100 \mathrm{~nJ}$, excitation pulses were centered at $390 \mathrm{~nm}$ and focused on the sample with a spot

21 size of $\sim 100 \mu \mathrm{m}$. The fluorescence setup (57) is based on Kerr-gating and $\mathrm{CS}_{2}$ was used as a

22 Kerr medium, yielding a temporal response of $\sim 1$ ps. For the absorption experiments the 
1 excitation pulses were polarized at magic angle with respect to the white light continuum probe

2 pulses to avoid photoselection effects. The sample ( $\sim 200 \mu \mathrm{M}$ of $C \nu \mathrm{FAP}$ containing FAD) was

3 circulated through a $1 \mathrm{~mm}$ optical path length cell of local design at a speed sufficient to allow

4 sample renewal between shots. For the experiments in the presence of substrate, as-purified

5 samples were used (fatty acid solubility limitations prohibited the presence of significantly

6 supra-stoichiometric amounts of substrate) and total absorbed photons during the experiments

7 were limited to less than $\sim 20 \%$ of the flavins, as verified by absorption spectroscopy.

8

\section{$9 \quad$ Excitation power dependence}

10 For experiments on the dependence of the transient absorption signal on the excitation power

11 using picosecond pulses, the 1-mm thick $\beta$-barium borate (BBO) crystal used for frequency

12 doubling of the 780 -nm fundamental beam was replaced by a custom $20-\mathrm{mm}$ thick BBO crystal

13 (Eksma). In this way, the output energy was distributed over a 4-ps interval due to the group

14 velocity mismatch between the 780-nm and 390-nm beams (58). Under conditions allowing a

15 pulse energy up to $1.4 \mu \mathrm{J}$ at the level of the sample, the FWHM was found to be $\sim 2.7 \mathrm{ps}$. Under

16 these conditions, at $1.4 \mu \mathrm{J}$ some pre-continuum was generated in the sample, leading to modest

17 distortion of the transient spectra up to $\sim 500 \mathrm{~nm}$. Results are shown in fig. S28.

19 Multi-scale transient infrared absorption spectroscopy

20 Time-resolved visible pump mid-infrared (IR) probe spectroscopy on the picosecond to

21 microsecond timescale was performed with a system based on two independent femtosecond

22 laser amplifiers (59). Pump pulses centered at $400 \mathrm{~nm}$ are generated by frequency doubling of 
$1 \quad 1-\mathrm{kHz} 150-\mathrm{fs} 800-\mathrm{nm}$ pulses using a first regenerative amplifier (Hurricane, Spectra-Physics).

2 The output of a second 1-kHz Titanium:Sapphire regenerative amplifier (LIBRA-HE, Coherent)

3 allowed to generate IR probe pulses by difference frequency mixing of the signal and idler pulses

4 delivered by an optical parametric amplifier. For the present experiments, the pulses were 5 centered at $2350 \mathrm{~cm}^{-1}$ and have a spectral width (FWHM) of $170 \mathrm{~cm}^{-1}$.

6 Time delays between pump and probe pulses were rapidly scanned from picoseconds to 7 microseconds thanks to a method combining Arbitrary-Detuning ASynchronous OPtical

8 Sampling (AD-ASOPS) $(32,60)$ and appropriate selection of amplified pulses that is described 9 in details in reference (33). This electronically-controlled approach allowed a very broad time 10 delay distribution without any moving part, while the temporal resolution can be as good as 200

11 fs. To enhance the signal-to-noise ratio on the picosecond timescale here we chose to average

12 spectra over 50-ps time bins, effectively limiting the time resolution.

Pump pulses were chopped mechanically at a frequency of $500 \mathrm{~Hz}$. The pump pulse

14 energy was $700 \mathrm{~nJ}$, and the angle between pump and probe polarizations was set to $90^{\circ}$. The

15 transmitted IR probe spectra were measured with a CCD by chirped-pulse up-conversion (61).

16 IR spectra were calibrated by comparison to atmospheric absorption lines (61). The part of the

17 set-up involving IR light was placed under dry air to limit the amplitude of these absorption

18 lines, and in particular those of atmospheric $\mathrm{CO}_{2}$.

19 Pump and probe pulses were focused on the sample on a $120-\mu \mathrm{m}$ FWHM spot. The 20 sample ( $\sim 2 \mathrm{mM}$ flavin concentration) was placed between two round $\mathrm{CaF}_{2}$ windows separated

21 by a $100 \mu \mathrm{m}$ thick spacer that leaves a circulation channel in the center, in a Harrick Scientific

22 Products DLC-M25 mount. The sample was continuously circulated using a peristaltic pump

23 connected to the cell so that the irradiated sample was nearly refreshed between two subsequent 
1 laser shots. The temporal overlap between pump and probe pulses is determined using the signal

2 of a GaAs wafer.

3 Each differential absorption plot shown in Fig 2C corresponds to the average of 33000 laser

4 shots. Baseline fluctuations were removed using a polynomial fit outside of the $\mathrm{CO}_{2}$ band and

5 the average baseline at two negative time delays ( $-5 \mathrm{~ns}$ and $-1 \mathrm{~ns})$ was subtracted from all spectra,

6 thus removing any contribution from the non-refreshed fraction of the sample. Both visible and

7 infrared transient spectra data sets were analyzed using Glotaran (62).

\section{$9 \quad$ Microcrystal growth and injection for TR-SFX}

10 Crystallization screening was performed under red-light conditions at $8^{\circ} \mathrm{C}$ using 96 -wells plates

11 in batch condition. Screening was done by mixing $20 \mu \mathrm{L}$ of $C v \mathrm{FAP}$ in solution $1(150 \mathrm{mM} \mathrm{NaCl}$,

$1210 \mathrm{mM}$ Tris $\mathrm{pH} 8.0,5 \%(\mathrm{w} / \mathrm{v})$ glycerol) at different concentrations $\left(3-12 \mathrm{mg} \mathrm{mL}^{-1}\right)$ with $20 \mu \mathrm{L}$

13 of mother liquor containing $100 \mathrm{mM}$ Na citrate pH 5.5, $10 \mathrm{mM}$ spermidine and PEG 4000 (12

14 to $28 \% \mathrm{w} / \mathrm{v})$. A 1:1 mixture of $19 \%(\mathrm{w} / \mathrm{v})$ PEG 4000, $0.1 \mathrm{M}$ sodium citrate $\mathrm{pH} 5.5,10 \mathrm{mM}$

15 spermidine (solution 2) and solution 1 at a protein concentration of $6 \mathrm{mg} \mathrm{mL}^{-1}$ yielded two types

16 of crystals, hereafter referred to as needle and diamond crystals based on their respective shapes.

17 Efforts to obtain only one crystal form were not successful. The diffraction quality of needle

18 shaped crystals $\left(30 \times 3 \times 3 \mu \mathrm{m}^{3}\right)$ was assessed at the microfocus beamline ID23-2 (63) of the

19 ESRF (Grenoble) at room temperature (RT; using MicroRT ${ }^{\mathrm{TM}}$ mounts from MiTeGen) and at

$20100 \mathrm{~K}$ (in a cryo-loop) after buffer exchange by centrifugation and resuspension in $20 \%$ (v/v)

21 glycerol, 23\% (w/v) PEG 4000, $100 \mathrm{mM} \mathrm{Na}$ citrate $\mathrm{pH} 5.5$ and $10 \mathrm{mM}$ spermidine. The

22 diffraction limit, as judged in the program Dozor implemented in MeshAndCollect data- 
1 collection workflow (64) was $2.3 \AA$ at $100 \mathrm{~K}$ and $2.6 \AA$ at room temperature. For TR-SFX

2 experiments, large quantities of microcrystals were generated using the batch method by gently

3 mixing $4 \mathrm{~mL}$ of solution 1 at a protein concentration of $6 \mathrm{mg}$ in $\mathrm{mL}^{-1}$ with $4 \mathrm{~mL}$ of solution 2 at

$4 \quad 8^{\circ} \mathrm{C} .1 .5$ grs of $C \nu \mathrm{FAP}$ was crystallized using this approach. Batches consisting mostly of needle

5 shaped crystals $\left(100-200 \times 2-5 \times 2-5 \mu \mathrm{m}^{3}\right)$ were selected and stored at $4^{\circ} \mathrm{C}$. Prior to injection,

6 they were reduced in size to about $10 \times 2-5 \times 2-5 \mu \mathrm{m}^{3}$ (fig. S29) by flowing a slurry containing

$720 \%(\mathrm{v} / \mathrm{v})$ microcrystals at $2.5 \mathrm{~mL} \mathrm{~min}^{-1}$ successively through a $20 \mu \mathrm{m}$ and a $10 \mu \mathrm{m}$ stainless

8 steel filter in tandem. After filtering, the crystal suspension was concentrated to $60 \%$ (v/v) by

9 centrifugation and removal of the supernatant and placed in a stainless steel sample syringe for

10 injection. The syringe was installed on an anti-settling device (65) equipped with a Peltier

11 element-cooled syringe holder at $4^{\circ} \mathrm{C}$. The crystals were injected with a gas dynamic virtual

12 nozzle (GDVN (66)), using sample capillaries with an inner diameter of 75 or $100 \mu \mathrm{m}$, into the

13 microfocus vacuum chamber of the CXI end station (67) of the Linac Coherent Light Source

14 (LCLS) at the SLAC National Accelerator Laboratory. The sample was injected at flow rates

15 between 35 and $55 \mu \mathrm{L} \mathrm{m^{-1 }}$ in a liquid jet of about $5 \mu \mathrm{m}$ in diameter.

17 Picosecond pump-laser excitation of FAP microcrystals in TR-SFX experiments

$18 C v$ FAP microcrystals $\left(\sim 10 \times 4 \times 4 \mu \mathrm{m}^{3}, P 2_{1}\right.$, two molecules $(\mathrm{A}, \mathrm{B})$ in the asymmetric unit) were 19 photoexcited with pulses of a Ti:sapphire laser (400 nm wavelength; $11 \mu \mathrm{J} /$ pulse, circularly 20 polarized). The pulse length was 4 ps (FWHM), achieved by detuning the pulse compressor. The 21 pump-laser beam of Gaussian shape was focused to a spot of $155 \mu \mathrm{m}$ (FWHM), resulting in a 22 peak power density of $10 \mathrm{GW} / \mathrm{cm}^{2}$. Under these excitation conditions, and assuming an 23 extinction coefficient of $9250 \mathrm{M}^{-1} \mathrm{~cm}^{-1}$ (estimated from Fig. 3A in Sorigué et al. 2017 under the 
1 assumption that the extinction coefficient at $467 \mathrm{~nm}$ is $11300 \mathrm{M}^{-1} \mathrm{~cm}^{-1}$ ) 2.8 photons were

2 absorbed on average per FAD chromophore. The number of absorbed photons in the front and

3 the rear of the crystals (68) do not deviate considerably from the average (fig. S30) because of

4 the large penetration depth of $400 \mathrm{~nm}$ light into the FAP crystals used (1/e penetration depth at

$5400 \mathrm{~nm}$ is $47 \mu \mathrm{m}$, see fig. S30). Excitation-power dependent transient absorption spectroscopy

6 carried out on $C \nu$ FAP in solution at the pump-power density $\left(10 \mathrm{GW} / \mathrm{cm}^{2}\right)$ of the TR-SFX

7 experiment (fig. S28) showed that multi-photon processes had decayed by the first TR-SFX time

8 point $(20 \mathrm{ps})$.

$10 \quad$ TR-SFX data collection and online monitoring

11 SFX data were collected (LT59, 22, 24 and 25 Nov 2018) in the microfocus chamber of CXI

12 with the LCLS producing X-ray pulses (nominal photon energy $9.5 \mathrm{keV}$, pulse length $23 \mathrm{fs}$ ) at

13 a repetition rate of $120 \mathrm{~Hz}$. The X-ray beam was focused to $1 \mu \mathrm{m} \times 1 \mu \mathrm{m}(\mathrm{FWHM})$, the nominal

14 pulse energy at the sample position was $\sim 1.3 \mathrm{~mJ}$, taking into account a beamline transmission of

$15>60 \%$. Data were acquired with the CSPAD detector (69) operating in a dual-gain mode. During

16 data collection, the hutch lights were switched off. On-line monitoring of the diffraction data,

17 such as estimation of the hit rate, the fraction of multiple hits and of the pixel saturation and

18 diffraction resolution, was carried out using the CFEL-ASG Software Suite (CASS) (70). TR-

19 SFX data were recorded according to a 400-nm pump (see above) - X-ray probe scheme, with

20 pump-probe delays of 20 ps, 900 ps, 300 ns and $2 \mu$ s. Two dark images without laser excitation

21 and 15 light images with laser excitation were collected in a pseudo-randomly interleaved

22 fashion. 


\section{SFX data processing}

3 On-line and off-line hit finding and dark vs. light image-sorting (without and with pump-laser

4 excitation, respectively) was performed using NanoPeakCell (71). Hit-finding parameters were

5 adjusted after visual inspection of the first diffraction patterns using the NanoPeakCell graphical

6 interface. A total of 8,141,555 images were collected for all five datasets (dark, light_20ps,

7 light_900ps, light_300ns, light_2 $\mu \mathrm{s}), 1,001,248$ of which were identified as hits, corresponding

8 to an overall hit-rate of $12.3 \%$.

CrystFEL v.0.8.0 (72) was used for detector geometry optimization, indexing (Xgandalf

10 (73)), integration (rings-grad method) and merging with the Monte Carlo (MC) algorithm

11 process_hkl. MC averaging included the scaling option. The sample-to-detector distance was

12 refined iteratively until the unit cell parameters displayed a Gaussian distribution (74). The

13 overall indexing rate (indexed images / hits) was about $25 \%$. A resolution cutoff of $2.0 \AA$ was

14 chosen based on acentric moments of the E distribution except for the light_ $2 \mu$ s dataset which

15 was cut at $2.2 \AA$ resolution. The space group is $P 2_{1}$, with two molecules (A, B) in the asymmetric

16 unit. SFX data processing statistics are shown in (Table S2).

17 In view of the large number of images used in the data sets, the high values of $R_{\text {split }}$ 18 indicate an underlying problem. We explored different space groups but the problem remained, 19 even when a lower symmetry was used, suggesting inherent disorder in the data as the 20 fundamental issue. There are several possible types of disorder that could affect the SFX data.

21 These include inherent variability in the microcrystals, which we cannot exclude at this time, as

22 well as some particular complications arising from the cell parameters of our FAP microcrystals: 
$1 a=61.4 \AA, b=60.0 \AA, c=182.9 \AA$ and $\alpha=90^{\circ}, \beta=90.6^{\circ}, \gamma=90^{\circ}$. Thus, not only are the cell

2 parameters close to orthorhombic, but also $a \approx b \approx 1 / 3 c$. This results in an indexing ambiguity, as

3 it is possible for an indexing algorithm to confuse the three axes. In addition, there is the

4 possibility of actual pseudomerohedral twinning in the crystals. All of these possible

5 complications would result in a high $\mathrm{R}_{\text {split }}$ and noisy maps. At present, none of our attempts at

6 resolving this static disorder (whether real or apparent) by reindexing of individual images based

7 on their correlation with the rest of the data set, twin refinement, or the screening of various

8 indexing schemes at the data processing stage, have resulted in a satisfactory solution.

\section{SFX dark structure solution and refinement}

11 The microcrystals used for SFX are of another crystal form than those used for the conventional

12 crystallography experiments; specifically, their space group is $P 2_{1}$ with two molecules in the

13 asymmetric unit as opposed to $I 222$ with a single molecule in the asymmetric unit for most of

14 the synchrotron structures (the R451K mutant crystallized in $P 2{ }_{1}{ }_{1} 2_{1}$ (one molecule in the

15 asymmetric unit) with very similar unit cell parameters as the $P 2{ }_{1}$ SFX crystal form). The dark

16 structure corresponding to the data set without laser excitation was phased by molecular

17 replacement with Phaser (75) using as a search model the synchrotron ' $100 \mathrm{~K}$ dark' structure of

18 FAP described in this study devoid of all cofactors and water molecules and with atomic B-

19 factors reset to $30 \AA^{2}$. This resulted in an unambiguous solution with two molecules in the

20 asymmetric unit and an excellent electron density map showing clear density for the FAD

21 cofactor as well as the fatty acid molecules. Structure refinement was performed using Refmac5

22 (46) and included positional and isotropic individual B-factor refinement in reciprocal space (25-

$232 \AA$ A resolution range) with local NCS restraints. Model building and real space refinement were 
1 performed using Coot (47). In the initial model, the isoalloxazine ring of the oxidized FAD

2 cofactor was constrained to be planar. $m F_{\text {obs }}-D F_{\text {calc }}$ peaks clearly indicated a significant

3 deviation from planarity (fig. S6). Therefore, once refinement of the protein moiety, waters and

4 substrate molecules was completed, the restraints of the isoalloxazine ring were relaxed by

5 increasing the estimated standard deviation of the isoalloxazine torsion angles in the .cif file. In

6 the final model (PDB accession code 6ZH7), the isoalloxazine ring of the FAD cofactor is clearly

7 bent (C4-N5-N10-C9 dihedral angle (butterfly bending angle), deviating from planarity by $14.3^{\circ}$

8 in molecule A and by $11.7^{\circ}$ in molecule B; Fig. 1E, fig. S7). Figures were made with PyMOL

9 (76). SFX data-structure refinement statistics are shown in table S2.

11 Calculation of difference Fourier maps between SFX data sets with and without laser

\section{2 excitation}

13 To assess structural changes induced by illumination, q-weighted (77) difference Fourier

14 electron density maps $\left(F_{\mathrm{obs}}{ }^{\text {light_20ps }}-F_{\mathrm{obs}}{ }^{\text {dark }}, F_{\mathrm{obs}}{ }^{\text {light_900ps }}-F_{\mathrm{obs}}{ }^{\text {dark }}, F_{\mathrm{obs}}^{\text {light_300ns }}-F_{\mathrm{obs}}{ }^{\text {dark }}\right.$,

$15 F_{\mathrm{obs}}^{\text {light_2us }}-F_{\mathrm{obs}}^{\text {dark }}$ ) were calculated in CNS (78) using scripts published in Wickstrand et al.

16 (79) and the SFX dark-state structure for phasing. The maps are shown in fig. S31 for each of

17 the two FAP molecules present in the asymmetric unit (A and B). As described in the main text,

18 the calculated q-weighted difference electron density maps show a different extent of

19 decarboxylation of the fatty acid at the various time delays, as well as various positive and

20 negative peaks throughout the active site and beyond. The maps contain some differences

21 between the two molecules in the asymmetric unit, suggesting either differences in the structural

22 change upon illumination or simply the maps' noise level. We therefore also averaged the maps

23 using a procedure based on local averaging (80). This resulted in the maps shown in Fig. 5. 
2 The model for the Molecular Dynamics (MD) simulations was built using the '100 K dark'

3 structure of FAP obtained in this work. The FAD cofactor in the oxidized state and two stearic

4 acids (STE) present in the crystal structure were included in the model. The simulations included

5 protein residues within a $24 \AA$ sphere, centered on the N5 atom of the bound FAD cofactor in

6 the crystal structure. Protein atoms between 20 and $24 \AA$ from the sphere's center were

7 harmonically restrained to their experimentally determined positions. Protonation states of

8 histidines were assigned by visual inspection and ideal stereochemistry; protonation states of

9 other residues were assigned using PROPKA $(81,82)$. In addition to crystal waters, a 71- $\AA$ cubic

10 box of water was overlaid, and waters overlapping with the protein, FAD, two STE's and crystal

11 water molecules were removed. Periodic boundary conditions were assumed; i.e. the entire 71-

$12 \AA$ box was replicated periodically in all directions. All long range electrostatic interactions were

13 computed by the particle mesh Ewald method (83). No counterions were included as the system

14 was electrically neutral. MD simulations were performed at constant temperature (300K) and

15 pressure, after 200 ps of thermalization. The CHARMM36 force field $(84,85)$ was used for the

16 protein and the modified version of the TIP3P water model (85-87). The force field parameters

17 for the FAD ox cofactor developed recently were used (27). Calculations were done with the

18 NAMD program (88). One hundred nanoseconds of MD simulations were performed.

\section{Quantum chemistry calculations}

21 The computations were performed using the Firefly software (89), which is partially based on

22 the US GAMESS code (90). A series of molecular models was employed in the calculations; all 
1 models were prepared using the coordinates of the ' $100 \mathrm{~K}$ dark' crystal structure. Geometries

2 were optimized using the density-functional theory restricted or unrestricted formalism with the

3 B3LYP functional $(91,92)$ supplemented with the dispersion energy correction D3 (93). The cc-

4 pvdz basis set (94) was used throughout. During geometry optimization, some atoms were

5 constrained (fig. S16) to mimic the structural relaxation of the active site being part of the protein

6 structure. The vertical and adiabatic electron affinities and ionization potentials were computed

7 using the R-/U-B3LYP energy calculations. The excitation energies were computed with the

8 XMCQDPT2 method (95) applying a denominator shift for intruder-state avoidance

9 (edshft=0.02) (96). The zero-order CASSCF wave functions were obtained using a state-

10 averaging procedure with equal weighting for all states included in the calculations (97). The so-

11 called minimum-size active space CASSCF calculations were performed for the FAP active site

12 following the approach previously extensively tested and applied for calculations of various

13 chromophores in proteins (98-102). The results obtained the FAP modes with different active

14 spaces and numbers of states were compared to evaluate consistency of the predicted excitation

15 energies. All excitation energies that are compared were obtained with the identical active space

16 of the flavin molecular orbitals. The generalized Mulliken-Hush scheme (103) was used in

17 conjunction with the XMCQDPT2 calculations to derive electronic couplings as previously

18 suggested for the bacteriorhodopsin active site (102).

\section{Comparison of GMC oxidoreductase sequences}

21 Sequences of 14 FAPs and 16 other GMC oxidoreductases that do not group with FAPs in a

22 phylogenetic tree were retrieved from various databases (table S4). FAP sequences were the 23 characterized $C v \mathrm{FAP}$ and Chlamydomonas FAP plus 12 other putative FAPs from various algal 
1 groups. The non-FAP GMC oxidoreductases were from a wide variety of organisms. Protein

2 sequences were aligned using the Muscle algorithm (104) and alignment was visualized and

3 inspected with SeaView (105). Logo sequences were generated using WebLogo.

5 Supplementary Text

6 S1. Study of FAD bending by low-dose X-ray crystallography, microspectrophotometry

7 and molecular dynamics

8 FAD reduction occurs during FAP X-ray exposure (with a half dose of $40 \mathrm{kGy}$ ) as shown by 20

9 X-ray dose-dependent in crystallo UV-Vis absorption microspectrophotometry scans (fig. S3),

10 i.e. well below the dose of the ' $100 \mathrm{~K}$ dark' data (154 kGy). However, a dark-state low-dose (38

$11 \mathrm{kGy}) 1.9 \AA$ Å resolution structure determined of FAP data collected at a synchrotron ('RT dark

12 LD') (fig. S4A and table S1) and a Raman microspectrophotometry experiment performed at

$13100 \mathrm{~K}$ at X-ray doses from 0 to $1.9 \mathrm{MGy}$ (fig. S4B,C) suggest that FAD is in a bent conformation

14 in the initial oxidized state (bending angle is $13.7^{\circ}$ in the 'RT dark LD'). FAD bending in the

15 oxidized state is also supported by a molecular dynamics simulation using a recently developed

16 flavin force field (27) that yielded an oxidized FAD cofactor bent on average by about $20^{\circ}$ (fig.

17 S5A,B).

19 S2. Band assignment in FTIR spectroscopy

20 The Light - minus - Dark difference spectra recorded at 100 or $150 \mathrm{~K}$ (Fig. 4A) display

21 vibrational contributions from all groups affected by the light-induced reaction. Negative bands

22 correspond to the Dark state and positive ones to the Light state. Bands from the protein, FAD, 
1 the substrate and products are expected to contribute to these FTIR difference spectra. To

2 identify contributions from the substrate and the reaction products, we substituted the native

3 substrate by ${ }^{12} \mathrm{C}$ or $1-{ }^{13} \mathrm{C}$-labeled palmitate, as described in the Materials and Methods section,

4 and compared the spectra (Fig. 4A, spectra $i$ and $i i$ ). Only IR modes involving the carboxyl group

5 of the substrate and products are expected to be significantly affected by this labeling. Therefore,

6 they can be identified in a ${ }^{12} \mathrm{C}$ - minus $-{ }^{13} \mathrm{C}$ difference spectrum calculated from spectra recorded

7 at $100 \mathrm{~K}$ (Fig. 4A, spectrum iii) and from spectra recorded at $150 \mathrm{~K}$ (Fig. 4A, spectrum iv). In

8 addition, downshifts of the band frequencies are expected upon ${ }^{13} \mathrm{C}$ - labeling. In spectra $i i i$ and

$9 i v$, the negative bands at 1541 and $1391 \mathrm{~cm}^{-1}$ are shifted to 1510 and $1371 \mathrm{~cm}^{-1}$, respectively,

10 upon $1{ }^{13} \mathrm{C}$-labeling, and are therefore assigned to the $v_{\mathrm{as}}\left(\mathrm{COO}^{-}\right)$and $v_{\mathrm{s}}\left(\mathrm{COO}^{-}\right)$IR modes of ${ }^{12} \mathrm{C}$ -

11 palmitate. The modes frequencies show that the fatty acid is deprotonated in the active site. They

12 appear as two thin and intense bands, indicating a very well defined position of the substrate in

13 the protein. In addition, the frequencies of the $v_{\text {as }}\left(\mathrm{COO}^{-}\right)$and $v_{\mathrm{s}}\left(\mathrm{COO}^{-}\right)$modes are lower than

14 those of carboxylate groups in solution (106), which indicates a strong interaction with positive

15 charges within the protein. This is in agreement with the substrate position identified in the ' 100

$16 \mathrm{~K}$ dark' synchrotron structure, showing a clear interaction of the substrate carboxylate group

17 with the side chain of R451 and with carbon C6 of the isoalloxazine ring of FAD (Fig. 1).

The $v_{\text {as }}\left(\mathrm{CO}_{2}\right)$ mode of the $\mathrm{CO}_{2}$ product of the reaction is clearly identified at $2340 \mathrm{~cm}^{-1}$

19 at $100 \mathrm{~K}$ (Fig. 4A panel $v$ ). The frequency is lower than that observed for $\mathrm{CO}_{2}$ in solution (2342

$\left.20 \mathrm{~cm}^{-1},(31)\right)$ and similar to that observed upon $C v \mathrm{FAP}$ illumination at room temperature by TR-

21 IR in the time window from 100 ps to $1 \mu$ s (Fig. 2 C,E and fig. S9). For samples with $1{ }^{13} \mathrm{C}-$

22 palmitate, it is downshifted by $-66 \mathrm{~cm}^{-1}$ to $2274 \mathrm{~cm}^{-1}$ as expected for ${ }^{13} \mathrm{CO}_{2}(107)$. 
At $150 \mathrm{~K}$, the intensity of the $v_{\text {as }}\left(\mathrm{CO}_{2}\right)$ mode is very low (Fig. 4A panel v). Between 1800

2 and $1200 \mathrm{~cm}^{-1}$, bicarbonate in solution is characterized by two main IR modes, the $v_{\text {as }}(\mathrm{CO})$ and

$3 v_{\mathrm{s}}(\mathrm{CO})$ modes at $1650-1620 \mathrm{~cm}^{-1}$ and $1365-1355 \mathrm{~cm}^{-1}$, respectively, and by a small amplitude

$4 \delta(\mathrm{COH})$ mode at $1300-1250 \mathrm{~cm}^{-1}(108,109)$. Upon ${ }^{13} \mathrm{C}$ labeling, frequency downshifts of -64

5 and $-25 \mathrm{~cm}^{-1}$ were reported for the $v_{\mathrm{as}}(\mathrm{CO})$ and $v_{\mathrm{s}}(\mathrm{CO})$ modes of bicarbonate in solution. The

6 frequency of the $v_{a s}(\mathrm{CO})$ and $v_{s}(\mathrm{CO})$ modes and the frequency shifts upon ${ }^{13} \mathrm{C}$-labeling are

7 sensitive to the interactions (hydrogen bonding, ionic) formed by bicarbonate with its

8 environment (109). In the ${ }^{12} \mathrm{C}-$ minus $-{ }^{13} \mathrm{C}$ difference spectrum recorded with $\mathrm{FAP}$ at $150 \mathrm{~K}$

9 (Fig. 4A panel $i v$ ), positive bands at 1646 and $1352 \mathrm{~cm}^{-1}$ are downshifted to 1614 and $1318 \mathrm{~cm}^{-}$

$10{ }^{1}$ upon $1-{ }^{13} \mathrm{C}$-palmitate labeling. The bands' frequency and shifts upon ${ }^{13} \mathrm{C}$-labeling are fully

11 consistent with contributions of bicarbonate. The FTIR data show that formation of bicarbonate

12 dominates over formation of $\mathrm{CO}_{2}$ after FAP illumination at $150 \mathrm{~K}$.

Contributions from protein side chain modes are also expected in the FTIR difference

14 spectra. In particular, the cysteine sulfhydryl group contributes by its $v(\mathrm{SH})$ mode in a specific

15 region of the FTIR spectra between 2700 and $2480 \mathrm{~cm}^{-1}$, depending on the interactions formed

16 by the sulfhydryl group and its environment $(110,111)$. FTIR spectra in the spectral range where

17 cysteine $v(\mathrm{SH})$ vibrations contribute show that, upon illumination of $C v \mathrm{FAP}$ at 100 or $150 \mathrm{~K}$, a

18 band shift occurs from $\approx 2540$ (dark) to $\approx 2550 \mathrm{~cm}^{-1}$ (light) (Fig. 4 A panel vi). This indicates

19 that a SH group is present at 100 and $150 \mathrm{~K}$, both in the Dark and Light states and that it

20 undergoes a slight change in hydrogen bonding interactions $(110,111)$. The lack of a negative

21 band in this region suggests that there is no stable cysteine deprotonation after illumination at

22 cryogenic temperatures. 
Tyrosine side chains contribute with an intense $v_{19}(\mathrm{CC})$ mode at $1515 \mathrm{~cm}^{-1}$ in the

2 infrared, which is sensitive to the Tyr protonation state and appears at $1495-1500 \mathrm{~cm}^{-1}$ for

3 tyrosinate $(106,112)$. Spectra recorded from the WT and the Y466F mutant are highly similar

4 in this spectral region (fig. S26A). This rules out significant contribution of the Y466 side chain

5 mode in the $1515-1495 \mathrm{~cm}^{-1}$ region of the light-induced FTIR difference spectrum. This is a

6 strong indication that there is no stable deprotonation of Y466 after illumination at cryogenic

7 temperatures.

8 In an attempt to identify IR bands of the R451 side chain, we compared light-induced

9 FTIR difference spectra recorded at $100 \mathrm{~K}$ with WT FAP and with the R451K mutant (fig.

10 S26B). Unfortunately, only a very small-amplitude FTIR difference spectrum was obtained from

11 the R451K mutant, and the spectrum was very different from that obtained from the WT FAP.

12 This prevented any conclusion on the contributions of the R451 side chain to the spectrum

13 obtained from WT FAP. On the other hand, we can formulate hypotheses from the spectra

14 recorded with WT FAP in $\mathrm{H}_{2} \mathrm{O}$, in $\mathrm{D}_{2} \mathrm{O}$, and with fully ${ }^{15} \mathrm{~N}$-labeled FAP (Fig. 4A, panels vii-

15 viii). The side chain of arginine is characterized by two intense IR modes, the $v_{\text {as }}$ and $v_{\mathrm{s}}\left(\mathrm{C}_{2} \mathrm{~N}_{3} \mathrm{H}_{5}\right)$

16 guanidinium modes, at circa $1675 \mathrm{~cm}^{-1}$ and $1635 \mathrm{~cm}^{-1}$, respectively, in $\mathrm{H}_{2} \mathrm{O}$ (106). These modes

17 are downshifted by -65 and $-47 \mathrm{~cm}^{-1}$ in $\mathrm{D}_{2} \mathrm{O}$, respectively (113). These modes are highly

18 sensitive to interactions formed by the Arg side chain and its deprotonation, as detailed in

19 Braiman et al. (114). Characteristics of these bands are their large intensity in $\mathrm{D}_{2} \mathrm{O}$ as well as the

20 larger effect of H/D exchange compared to that expected for other IR modes contributing in the

$211700-1600 \mathrm{~cm}^{-1}$ region, i.e., mainly Amide I modes of peptide carbonyl groups (which are not

22 expected to shift by more than $10 \mathrm{~cm}^{-1}$ in $\mathrm{D}_{2} \mathrm{O}$ ). Therefore, they can be distinguished from other

23 contributions by comparing spectra recorded in $\mathrm{H}_{2} \mathrm{O}$ and $\mathrm{D}_{2} \mathrm{O}$ and especially in calculated $\mathrm{H}_{2} \mathrm{O}$ 
- minus - $\mathrm{D}_{2} \mathrm{O}$ spectra, such as those presented in Fig. 4A panel viii. If we cannot unambiguously

2 assign the Arg side chain modes in $\mathrm{H}_{2} \mathrm{O}$, due to possible overlap with other bands, we can

3 propose that the band observed at $1606 \mathrm{~cm}^{-1}$ in $\mathrm{D}_{2} \mathrm{O}$ is due to an arginine $v_{\mathrm{s}}$ (guanidium) mode.

4 Indeed, the negative band observed at $1606 \mathrm{~cm}^{-1}$ in $\mathrm{D}_{2} \mathrm{O}$ has no clear counterpart in the close-by

5 region in $\mathrm{H}_{2} \mathrm{O}$ : it therefore results from a downshift in $\mathrm{D}_{2} \mathrm{O}$ of at least $-30 \mathrm{~cm}^{-1}$. In addition, this

6 band is intense. To our knowledge, there is no other amino acid side chain that could show such

7 a contribution in $\mathrm{D}_{2} \mathrm{O}$ (and not in $\mathrm{H}_{2} \mathrm{O}$ ) in this spectral region. We can also exclude contributions

8 from Amide II modes at this frequency since Amide II modes contribute usually in the 1580 -

$9 \quad 1500 \mathrm{~cm}^{-1}$ region in $\mathrm{H}_{2} \mathrm{O}$, and are downshifted upon $\mathrm{H} / \mathrm{D}$ exchange by about $-100 \mathrm{~cm}^{-1}$ to the

$101500-1400 \mathrm{~cm}^{-1}$ region. The band at $1606 \mathrm{~cm}^{-1}$ is clearly a well defined, thin negative band

11 present in the spectrum recorded with samples in $\mathrm{D}_{2} \mathrm{O}$ and not in $\mathrm{H}_{2} \mathrm{O}$. This frequency value

$12\left(1606 \mathrm{~cm}^{-1}\right)$ is well above that expected for Amide II modes in $\mathrm{D}_{2} \mathrm{O}$. To further support the

13 proposed assignment, we produced fully ${ }^{15} \mathrm{~N}$-labeled FAP and recorded spectra with samples in

$14 \mathrm{H}_{2} \mathrm{O}$ and $\mathrm{D}_{2} \mathrm{O}$. The corresponding $\mathrm{H}_{2} \mathrm{O}-$ minus $-\mathrm{D}_{2} \mathrm{O}$ difference spectrum recorded at $100 \mathrm{~K}$

15 shows that the band at $1606 \mathrm{~cm}^{-1}$ is downshifted to $1597 \mathrm{~cm}^{-1}$ (Fig. 4 A panel viii). This strongly

16 supports the assignment of this band to an arginine side chain mode. In addition, it allows us to

17 exclude the assignment of this band to an Amide I mode, since, upon ${ }^{15} \mathrm{~N}$-labeling, the Amide I

18 bands are expected to shift only by a few wavenumbers $\left(-1\right.$ to $\left.-3 \mathrm{~cm}^{-1}\right)$ due to a small coupling

19 between the $v(\mathrm{C}=\mathrm{O})$ mode and the $\mathrm{NH}$ in plane bending mode. Although FAP ${ }^{15} \mathrm{~N}$-labeling

20 induces downshifts of bands of the $\mathrm{H}_{2} \mathrm{O}-$ minus $-\mathrm{D}_{2} \mathrm{O}$ spectrum in the $1703-1686 \mathrm{~cm}^{-1}$ region

21 and at $1644 / 1631 \mathrm{~cm}^{-1}$ (to $1638 / 1624 \mathrm{~cm}^{-1}$ ), we cannot assign some of these bands to arginine

22 side chain modes, since contributions from Asn or Gln side chains cannot be excluded in this

23 spectral range, as well as small downshifts of Amide I bands. However, the frequency and 
1 intensity of the band at $1606 \mathrm{~cm}^{-1}$, the effect of $\mathrm{H} / \mathrm{D}$ exchange and ${ }^{14} \mathrm{~N} /{ }^{15} \mathrm{~N}$ exchange on this

2 band altogether point to a contribution from an arginine side chain mode in $\mathrm{D}_{2} \mathrm{O}$ at $1606 \mathrm{~cm}^{-1}$.

3 This band is negative, with no clear positive counterpart. This is also clear in the $\mathrm{H}_{2} \mathrm{O}-$ minus -

$4 \quad \mathrm{D}_{2} \mathrm{O}$ difference spectrum of Fig. 4A spectrum viii calculated from spectra in vii. This is a strong

5 argument to infer a large change in properties of the Arg side chain after illumination of the

6 sample at $100 \mathrm{~K}$. Therefore, the FTIR data are consistent with a model in which an Arg side

7 chain is deprotonated after illumination at $100 \mathrm{~K}$. In their study of bacteriorhodopsin, Braiman

8 et al. assigned a weak IR band at circa $1555 \mathrm{~cm}^{-1}$ to deprotonated guanidium (114). This band

9 occurs in the region where peptide Amide II modes also contribute, so that any assignment would

10 be ambiguous in this region in Fig. $4 \mathbf{A}$ spectrum viii.

$\underline{\text { S3. Time-resolved fluorescence in solution on ps to ns timescales }}$

WT CVFAP

The fluorescence kinetics of the WT $C \nu \mathrm{FAP}$ in the presence of the native substrate(s) (Fig. 3A,

16 Fig. 7B and fig S25A,B) show that the substrate quenches the singlet excited state ${ }^{1} \mathrm{FAD}^{*}$,

17 shortening its lifetime from $\sim 6.5 \mathrm{~ns}$ (typical time constant for unquenched flavins (115)) to $~ 300$

18 ps (both in $\mathrm{H}_{2} \mathrm{O}$ and $\mathrm{D}_{2} \mathrm{O}$, see Fig. 3A). Interestingly, fluorescence signals recorded in the 19 presence of one or two native substrate molecules (see X-ray structures in Fig. 1) or even >10-

20 fold excess of added substrate still contain a non-negligible contribution of the slow phase

21 (corresponding to $\sim 15 \%$ of the recorded signal amplitude). This suggests that there is always a

22 certain fraction of enzymes that upon photoexcitation cannot undergo the productive fET step

23 (inactive proteins), but in which the ${ }^{1} \mathrm{FAD} *$ undergoes intersystem-crossing (ISC) to a non- 
1 productive triplet state ${ }^{3} \mathrm{FAD}^{*}$ (like in the absence of a substrate as shown previously (4)), which

2 then decays back to the ground state FAD in $\sim 80 \mu \mathrm{s}$. Noteworthy, competition between fET and

3 ISC in a homogeneous sample should lead to a monoexponential decay of the ${ }^{1} \mathrm{FAD} *$

4 fluorescence. It hence seems that the fate of ${ }^{1} \mathrm{FAD}^{*}$ is decided prior to the FAD excitation.

5 Reasons for the small fraction of inactive WT proteins can be diverse, ranging from an

6 overstabilization of the carboxylate by a transient bidentate interaction with R451, to an

7 incomplete occupation of the substrate binding site or an incomplete deprotonation of the

8 substrate therein.

$10 \quad$ CvFAP mutants

11 In the Y466F mutant, the time-resolved fluorescence signal (fig. S25B) is very similar to that of

12 the WT protein. Apart from a slightly increased relative amplitude of the slower phase

13 (indicative of a minor increase in the fraction of flavins that cannot undergo fET and eventually

14 leading to a slightly lower enzymatic activity; Fig. 7A), ${ }^{1}$ FAD* fluorescence quenching by ET

15 from the substrate seems to occur with the same kinetics in Y466F as in WT (i.e., in 300 ps).

Fluorescence signals of the R451 mutants (see Fig. 7B for R451K and fig. S25B for

$17 \mathrm{R} 451 \mathrm{~A})$ resemble those obtained for the WT $C \nu \mathrm{FAP}$ after the photocatalytic consumption of the

18 native substrate (fig. S25A), in spite of the fact that the substrates are still present at the catalytic

19 site (see Fig. 7D (R451K) \& fig. S26D (R451A)).

20 In the R451K mutant, ${ }^{1} \mathrm{FAD} *$ fluorescence decays significantly faster than in the WT

21 after the consumption of the substrate (Fig. 7B). Due to the systemic initial perturbations in the

22 signal caused by the detection system, we are not capable of distinguishing whether the decay is 
1 monophasic or biphasic in this case. A biexponential fit yields time constants of $\sim 300$ ps and $\sim 5$

2 ns, i.e., similar to those for the WT in the presence of substrate ( $300 \mathrm{ps}$ and $\sim 6.5 \mathrm{~ns})$, but with

3 an inverse amplitude ratio (i.e., $\sim 15 \%$ fast phase and $\sim 85 \%$ slow phase). A monoexponential fit

4 yields a time constant of $\sim 4.5 \mathrm{~ns}$ ( $v s . \sim 6.5 \mathrm{~ns}$ for WT after substrate consumption). If we assume

5 the decay is monoexponential and reflects two competing processes, fET and ISC, the rate

6 constant $k_{\mathrm{obs}}\left(=1 / \tau_{\mathrm{obs}}\right)$ is a sum of $k_{\mathrm{fET}}+k_{\mathrm{ISC}}$. For $\tau_{\mathrm{obs}}$ of $4.5 \mathrm{~ns}(\mathrm{R} 451 \mathrm{~K})$ and $6.5 \mathrm{~ns}$ (WT without

7 substrate, i.e., $100 \%$ ISC), we obtain $k_{\text {obs }}$ of $2.2 \times 10^{8} \mathrm{~s}^{-1}$ and $1.54 \times 10^{8} \mathrm{~s}^{-1}$, respectively. If we

8 further assume that the $k_{\mathrm{ISC}}$ is the same in WT and in the mutant (which may or may not be the

9 case; i.e., $k_{\mathrm{ISC}}=1.54 \times 10^{8} \mathrm{~s}^{-1}$ ), we obtain $k_{\mathrm{fET}}$ of $6.8 \times 10^{7} \mathrm{~s}^{-1}$, which converts into an intrinsic time

10 constant for fET of $\sim 15 \mathrm{~ns}$ in R451K. Since $k_{\mathrm{fET}}$ is $\sim 30 \%$ of $k_{\mathrm{obs}},{ }^{1} \mathrm{FAD} *$ should decay by $\sim 30 \%$

11 fET and $\sim 70 \%$ ISC in R451K. The tests of enzymatic activity (Fig. 7A), and the transient

12 absorption signals (fig. S25D) show that the R451K mutant is indeed partially active ( $45 \%$ of

13 WT, which would correspond to $\sim 38 \% \mathrm{fET}$, since fET yield in WT is $\sim 85 \%$ ). The small

14 difference in the two numbers (30\% vs. $38 \%$ ) could be explained in at least threeways: 1) $k_{\mathrm{ISC}}$

15 might be slightly slower in R451K than in WT, 2) the fET yield for the native substrates (in the

16 fluorescence experiment) may be slightly lower than for the added ones (in the activity test), or

17 3) the recovery of the catalytic site (steps 5 to 7 of our photocycle, Fig. 8) might be slightly faster

18 in the mutant than in the WT protein, which would not affect the yield of fET but it would

19 increase the apparent enzymatic activity under continuous illumination conditions (as it seems

20 to be the case for Y466F; see discussion in the supplementary text S4). In any case, the observed

21 decrease in both fET yield and enzymatic activity under steady-state illumination is likely due

22 to an increased FAD-substrate distance (from $3.2 \AA$ in WT to $4.6 \AA$ in $\mathrm{R} 451 \mathrm{~K}$ ) and to an 
1 'overstabilization' of the fatty acid carboxylate by the presence of the additional water molecule

2 Wat3 (see Fig. 7D).

In the R451A mutant, ${ }^{1} \mathrm{FAD} *$ fluorescence decays monophasically with a time constant

4 of $\sim 8 \mathrm{~ns}$ and no rapid process indicative of its quenching by productive fET is observed (fig.

5 S25B). Accordingly, the transient absorption data (fig. S25E) do not display any significant

6 evolution, which could be attributed to the bET reaction on the sub- $\mu$ s timescale, and the mutant

7 exhibits essentially no enzymatic activity (Fig. 7A). We propose that the inactivity of the R451A

8 mutant is primarily due to the 'wrong' protonation state of the substrate, which, in the absence

9 of a positive counterion on residue 451, is most probably protonated and thus cannot reduce the

10 photoexcited ${ }^{1} \mathrm{FAD} *$

11 In the C432S mutant, fluorescence of ${ }^{1} \mathrm{FAD}^{*}$ (Fig. 7B) decays apparently

12 monophasically in $\sim 6.0 \mathrm{~ns}$ (i.e., only slightly faster than in WT after consumption of the native

13 substrate $(\sim 6.5 \mathrm{~ns})$, indicating that fET does occur to a small extent in C432S). Partial

14 consumption of the native substrates (by 10 strong flashes at $470 \mathrm{~nm}$ ) slowed the fluorescence

15 decay down to $~ 6.2 \mathrm{ns,}$, addition of a five-fold excess of oleic acid, on the other hand, led to its

16 minor acceleration to $\sim 5.7 \mathrm{~ns}$ (not shown). Applying the same procedure for the estimation of

17 intrinsic fET rate as we used for R451K above, the time constants of $\sim 6.0 \mathrm{~ns}$ (C432S with native

18 substrates) and $\sim 5.7 \mathrm{~ns}$ (C432S in the presence of excess oleic acid) translate into $k_{\mathrm{fET}}$ of $1.3 \times 10^{7}$

$19 \mathrm{~s}^{-1}\left(\tau_{\mathrm{fET}}\right.$ of $\left.\sim 80 \mathrm{~ns}\right)$ and $2.1 \times 10^{7} \mathrm{~s}^{-1}\left(\tau_{\mathrm{fET}}\right.$ of $\left.\sim 50 \mathrm{~ns}\right)$, respectively, and fET yields of 8 and $12 \%$,

20 respectively. The latter two numbers are both consistent with the $\sim 10 \%$ activity of C432S

21 determined by membrane inlet mass spectroscopy. 
$3 \quad$ WT CVFAP

4 Based on our initial characterizations of the FAP photocycle by transient absorption

5 spectroscopy (4), we have chosen three characteristic wavelengths to monitor FAD-associated

6 spectral changes following its photoexcitation in WT and mutant $C \nu \mathrm{FAP}: 376 \mathrm{~nm}$, where $\mathrm{FAD}^{\bullet-}$

7 absorbs more strongly than oxidized FAD (observation of $\mathrm{FAD}^{\bullet-}$ formation and decay); $488 \mathrm{~nm}$,

8 where the $\mathrm{FAD}^{\bullet-}$ anion absorption is weaker than that of oxidized FAD (observation of FAD

9 absorption bleaching and its recovery upon $\mathrm{FAD}^{\bullet-}$ reoxidation); and finally $515 \mathrm{~nm}$, where

$10 \mathrm{FAD}^{\bullet-}$ absorbs more than oxidized FAD but less than the red-shifted re-oxidized FAD

11 (observation of $\mathrm{FAD}^{\bullet-}$ formation and its conversion into $\mathrm{FAD}_{\mathrm{RS}}$ on the sub- $\mu$ s timescale, and

12 the subsequent decay of $\mathrm{FAD}_{\mathrm{RS}}$ back to the "dark-state" FAD on the ms timescale).

Both fluorescence and transient absorption signals obtained for the truncated $C v \mathrm{FAP}$ in

$14 \mathrm{H}_{2} \mathrm{O}$ buffer (Fig. 3) are highly similar to those obtained previously for the full-length $C v$ FAP

15 (Fig. 5C and fig. S6 in (4)), indicating that the truncation of the protein does not affect its

16 photochemistry.

A change in $\mathrm{pH}$ (of the $100 \mathrm{mM}$ Tris Buffer) in the interval between 7.5 and 9.1 (i.e.,

18 around the enzymatic optimum according to fig. S3D in (4)) affected neither the kinetics, nor

19 the amplitude of any of the signals (fig. S10A,B).

Consumption of the native substrates by saturating flashes at $470 \mathrm{~nm}$ (in the absence of

21 added substrate) did, as expected, lead to a decrease in the amplitude of the signals recorded on

22 the ms timescale but their kinetics remained essentially unaffected (fig. S10C). 
On the contrary, the exchange of an $\mathrm{H}_{2} \mathrm{O}$ buffer to a $\mathrm{D}_{2} \mathrm{O}$ buffer had no impact on the

2 amplitudes of the observed signals but it did substantially slow down the kinetics of both

3 processes followed by transient absorption spectroscopy: the reoxidation of $\mathrm{FAD}^{\bullet-}$ to $\mathrm{FAD}_{\mathrm{RS}}$

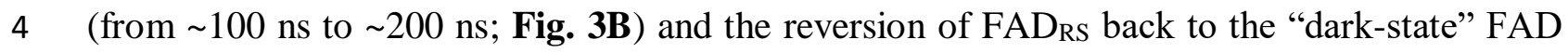

5 (from $\sim 3 \mathrm{~ms}$ to $\sim 10 \mathrm{~ms}$; Fig. 3C). For the former process $\left(\mathrm{FAD}^{\bullet-}\right.$ reoxidation), Heyes and

6 coworkers (24) reported a kinetic isotope effect $(K I E)$ of only $\sim 1.2$ (lifetimes of $\sim 180 \mathrm{~ns}$ and

$7 \sim 215 \mathrm{~ns}$ in $\mathrm{H}_{2} \mathrm{O}$ and $\mathrm{D}_{2} \mathrm{O}$, respectively) at $520 \mathrm{~nm}$ and concluded that ' $\mathrm{H}$-transfer events did not

8 have a major effect on the rate of formation of the red-shifted flavin intermediate'. The origin of

9 the deviation from our data (obtained at three different wavelengths, including 515 nm; see Fig.

10 3B) might be an insufficient time resolution in Ref. (24) that not only affected the faster kinetics

11 (in $\mathrm{H}_{2} \mathrm{O}$ ) more than the slower one (in $\mathrm{D}_{2} \mathrm{O}$ ) but also made it difficult to discriminate the rise in

12 a few hundreds of nanoseconds from the preceding much faster (step-like) rise due to $\mathrm{FAD}^{\bullet-}$

13 formation, that is not affected by the isotope exchange (compare Fig. 3A in Ref. (24) with our

14 Fig. 3B (signals at $515 \mathrm{~nm}$ )). The much stronger $K I E$ documented here argues for direct coupling

15 of bET to a $\mathrm{H}^{+} / \mathrm{H}^{\bullet}$ transfer event. Note that an indirect coupling due to, e.g., structural changes

16 in the substrate/flavin region induced by solvation of the protein in $\mathrm{D}_{2} \mathrm{O}$ instead of $\mathrm{H}_{2} \mathrm{O}$ cannot

17 be reconciled with the absence of $K I E$ on fET from the substrate to the excited flavin (Fig. 3A).

18 The absence of a $\mathrm{pH}$ effect in the interval between 7.5 and 9.1 (fig. S10A,B) indicates

19 that if these reaction steps (steps 3 and 5 in Fig. 8) involve PT (or HAT), the p $K_{\mathrm{a}}$ of the proton

20 or hydrogen atom donor lies outside of the tested $\mathrm{pH}$ interval (note that the $\mathrm{p} K_{\mathrm{a}}$ of the used Tris

21 buffer is $\sim 8.1$; as the total buffer concentration was constant in these experiments, the

22 concentration of its acidic and basic forms changed strongly with $\mathrm{pH}$, but this did not affect the

23 kinetics, indicating that Tris-buffer molecules are not directly involved). Finally, the lack of 
1 influence of the availability of a fresh substrate on the kinetics of FAD ${ }_{\mathrm{RS}}$ reversion to the "dark-

2 state" FAD (fig. S10C) suggests that the disappearance of the transient FAD red shift is not

3 associated with binding of a new substrate.

4

$5 \quad$ CvFAP mutants

6 Like in the case of fluorescence signals (fig. S25B), transient absorption changes in the Y466F

7 mutant on the sub-microsecond time scale reflecting bET/FAD ${ }_{\mathrm{RS}}$ formation (fig. S25C) also

8 resemble those obtained for the WT protein (Fig. 3B or fig. S10A), even though a moderate

9 acceleration of bET can be noticed in Y466F. Together with the almost identical FTIR signals

10 of WT and Y466F CvFAP (fig. S26A) and a high enzymatic activity of this mutant (Fig. 7A),

11 all our results suggest that tyrosine Y466 does not directly participate in any of the PT/HAT or

12 ET steps in the FAP photocycle. However, Heyes et al. (24) have shown that the decay of the

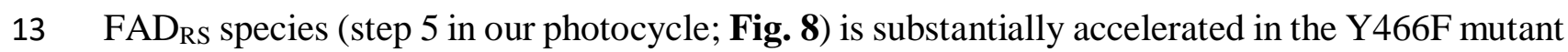

14 (we observed the same phenomenon - acceleration of the FAD ${ }_{\mathrm{RS}}$ decay from $\sim 3 \mathrm{~ms}$ in WT to

$15 \sim 300 \mu \mathrm{s}$ in $\mathrm{Y} 466 \mathrm{~F}$; data not shown). Heyes et al. suggest that it "might reflect differences in local

16 reorganization in the active site'. Alternatively, since the phenyl group of Y466 makes a

17 hydrogen bond to the backbone carbonyl oxygen of T430 (O-O distance of $2.6 \AA$ ), serving as a

18 "clasp" around the alkyl chain, one can also speculate that F466 (being incapable of such an

19 interaction) is likely more flexible than Y466 and that the missing "clasp" might facilitate egress

20 of the product chain through the tunnel (lined by the second FA) connecting to the solvent. This

21 could allow for a faster recovery of the active site after the photoreaction (steps 5 to 7 in our

22 photocycle; Fig. 8). According to fig. S10A in (24), the Y466F mutant seems to be a bit more

23 active than WT in the first minutes of the activity test (under continuous illumination). Given

24 that the quantum yield of fET is actually slightly lower in Y466F than in WT (the relative 
1 amplitude of the slower phase in the ${ }^{1} \mathrm{FAD}^{*}$ fluorescence decay corresponding to futile ISC is a

2 bit more pronounced in Y466F than in WT, see fig. S25B), this observation can only be

3 explained in terms of a faster recovery of the active site in Y466F (combined with a faster

4 photodegradation of the mutant protein).

Transient absorption changes recorded for the R451K and R451A mutants on the sub-

6 microsecond timescale are shown in fig. S25D and E, respectively. In the signals of the R451K

7 mutant, the amplitudes of the first kinetic phase (reflecting the reoxidation of $\mathrm{FAD}^{\bullet-}$ to $\mathrm{FAD}_{\mathrm{RS}}$ )

8 are considerably smaller than in WT $C \nu \mathrm{FAP}$ (corresponding to $\sim 30 \%$ bET, judging from the

$9 \quad$ signal at $488 \mathrm{~nm}$, fig. S25D), but this process seems to be markedly accelerated (from $\sim 100 \mathrm{~ns}$

10 in WT to $\sim 30 \mathrm{~ns}$ in R451K; fig. S25D). While the $515 \mathrm{~nm}$ signal remains constant after the initial

$11 \sim 30 \mathrm{~ns}$ phase, we observe further partial reversion of the negative absorption change at $488 \mathrm{~nm}$

12 with a time constant of a few microseconds. The additional phase at $488 \mathrm{~nm}$ most probably

13 corresponds to decay of free FAD triplet (characteristic ${ }^{3} \mathrm{FAD} *$ lifetime in solution is $\sim 3 \mu$ s; note

14 that the absorption change due to ${ }^{3} \mathrm{FAD} *$ should be much more pronounced at $488 \mathrm{~nm}$ than at

$15515 \mathrm{~nm}$; see fig. S6D in (4)). Remarkably, in the (dark) crystal structure of the R451K mutant

16 (Fig. 7D), the side chain of C432 changes its position so that its SH group recedes farther both

17 from the FAD (from $6.9 \AA$ to $9.6 \AA$ ) and from the $\mathrm{C} 2$ atom of the substrate (from $4.7 \AA$ to 9.2

$18 \AA$ ). If reduction of the alkyl radical were due to HAT from C432 followed by bET from FAD ${ }^{\bullet-}$

19 to the $\mathrm{C} 432$ radical (without large structural rearrangement following $\mathrm{CO}_{2}$ formation), one would

20 expect the reoxidation of $\mathrm{FAD}^{\bullet-}$ to slow down in the $\mathrm{R} 451 \mathrm{~K}$ mutant. The opposite observation

21 argues against both the hydrogen atom transfer from and the coupled back electron transfer to

22 the $\mathrm{C} 432$ residue as the mechanism of alkyl radical reduction by $\mathrm{FAD}^{\bullet-}$. On the other hand, the 
1 higher acidity of lysine should increase the driving force for a PT from the residue 451 (coupled

2 to bET from $\mathrm{FAD}^{\bullet-}$ ) to the alkyl radical in the arginine to lysine mutant.

In the case of the R451A mutant, we observe essentially only step-like absorption

4 changes at both selected wavelengths (fig. S25E), compatible with the formation of a protein-

5 bound FAD triplet (with a lifetime of $\sim 80 \mu$ s; see fig. S6E,F in (4)): the signal recorded at 488

6 nm very much resembles that recorded for the WT protein after consumption of the native

7 substrate (see fig. S6D in (4)). Yet, the signal at $515 \mathrm{~nm}$ in the R451 A mutant shows a significant

8 negative absorption change (instead of almost zero in WT after substrate consumption), which

9 is probably caused by a pronounced $(\sim 8 \mathrm{~nm})$ red shift in the 'dark state' FAD spectrum in R451A

10 (see fig. S24D or inset of fig. S25E). The minor initial phase seen in the $488 \mathrm{~nm}$ signal (fig.

11 S25E) likely reflects ISC from ${ }^{1} \mathrm{FAD} *$ to ${ }^{3} \mathrm{FAD} *(\sim 8 \mathrm{~ns}$; see fig. S25B).

Transient absorption changes at $488 \mathrm{~nm}$ recorded for the C432S mutant on the ns and $\mu \mathrm{s}$

13 timescales (fig. S25F) also resemble those recorded for the WT protein after consumption of the

14 native substrate (see fig. S6D,E in (4)), indicating that the formation and decay of ${ }^{3} \mathrm{FAD} *$ are the

15 dominating processes (compared to $\mathrm{WT},{ }^{3} \mathrm{FAD} *$ decay is mildly accelerated in $\mathrm{C} 432 \mathrm{~S}$ : from $\sim 80$

$16 \mu$ s to $\sim 30 \mu$ s; see lower inset of fig. S25F)(compared to WT, 3FAD* decay is mildly accelerated

17 in C432S: from $\sim 80 \mu$ s to $\sim 30 \mu$ s; see lower inset of fig. S25F). At $515 \mathrm{~nm}$ (where the signal WT

18 FAP w/o substrate in fig. S6D in (4) returns to almost zero after the initial ${ }^{1} \mathrm{FAD}^{*}$ decay phase

19 in a few ns), however, the signal remains markedly positive in C432S (with an amplitude

20 corresponding to $\sim 15 \%$ of WT with substrate) and most (about $2 / 3$ ) of this positive absorption

21 change decays in $\sim 3 \mathrm{~ms}$ (upper inset of fig. S25F), i.e., with the same kinetics as the FADRS

22 decays in the WT protein (see also Fig. 3D in (24)). As evidenced by cryo-trapping (Fig. 7C), 
$1 \mathrm{FAD}_{\mathrm{RS}}$ indeed forms in the C432S mutant and while we could not properly resolve the kinetics

2 of its formation, we attribute the $\sim 3 \mathrm{~ms}$ decay to its disappearance.

\section{$4 \quad$ S5. Time-resolved fluorescence spectroscopy of $C v$ FAP crystals}

5 Even though most crystalline proteins are biologically active, their reaction kinetics often differ

6 from the solution state $(116,117)$. In preparation for TR-SFX experiments, we assessed the

7 kinetics of FAP in the crystalline and solution state by carrying out fluorescence-decay

8 measurements. For solution experiments, the $6 \mathrm{mg} \mathrm{mL}^{-1}$ FAP solution was diluted to $0.375 \mathrm{mg}$

$9 \mathrm{~mL}^{-1}$ in buffer $(50 \mathrm{mM}$ Tris $\mathrm{pH} 8.5,100 \mathrm{mM} \mathrm{NaCl})$. Experiments on crystalline FAP were carried

10 out on a suspension of microcrystals $\left(1 \%(\mathrm{v} / \mathrm{v})\right.$; each about $10 \times 2-5 \times 2-5 \mu \mathrm{m}^{3}$ in size $)$ in

$1119 \%$ (w/v) PEG 4000, $100 \mathrm{mM}$ Na citrate $\mathrm{pH}$ 5.5, $10 \mathrm{mM}$ spermidine. Single photon counting

12 fluorescence experiments were performed using an excitation pulse $(400 \mathrm{~nm})$ delivered by

13 doubling (harmonic generator, SHG, APE) a femtosecond Ti:sapphire laser (800 nm, Coherent

14 Chameleon Ultra II, $80 \mathrm{MHz}, 180 \mathrm{fs}$ ) coupled to a pulse picker (APE, $4 \mathrm{MHz}$ ). Emission decays

were measured using an FT200 Picoquant spectrometer and analyzed by the FluoFit software.

16 By using a low excitation power $(100 \mathrm{nW})$, a small excitation beam size $(100 \mu \mathrm{m})$ and a large

17 volume (2 mL) of sample that was constantly stirred, fluorescence spectra could be acquired for

18 about 3 min without notable chromophore bleaching (as assessed by a UV-Vis absorption

19 spectrum). The measured emission decay of ${ }^{1} \mathrm{FAD}^{*}$ at $560 \mathrm{~nm}$ (fig. S15) was fitted by a

20 weighted-sum of three exponentials. For FAP in solution, the retrieved time constants

21 corresponding to three different FAD populations were $318 \mathrm{ps}( \pm 5 \mathrm{ps}), 980 \mathrm{ps}( \pm 30 \mathrm{ps})$ and 3.9

22 ns $( \pm 40 \mathrm{ps})$ with contributions of $76 \%, 16 \%$ and $8 \%$, respectively. The first population

23 corresponds to FAD undergoing efficient fET, the second to a population undergoing less 
1 efficient fET or being photobleached, and the last to a non-reactive population. For the crystal

2 suspension, similar time constants were retrieved (320 ps, $1.45 \mathrm{~ns}, 4.8 \mathrm{~ns})$ but with different

3 contributions $(62 \%, 14 \%, 24 \%)$, leading to an overall fluorescence decay that is slower in

4 crystals than in solution (fig. S15). To assess whether the decrease in the population undergoing

5 efficient fET in the crystal suspension (62\%) compared to solution (72\%) is due to the difference

6 in $\mathrm{pH}$ (5.5 in crystals versus 8.5 in solution), the experiment was repeated on a FAP solution

$7 \quad\left(0.375 \mathrm{mg} \mathrm{mL}^{-1}\right.$ protein concentration) in the crystal buffer (19\% (w/v) PEG 4000, $100 \mathrm{mM} \mathrm{Na}$

8 citrate $\mathrm{pH} 5.5,10 \mathrm{mM}$ spermidine). The contribution of the population with a fluorescence decay

9 of 322 ps was $75 \%$, indicating that it is the crystal packing and not the $\mathrm{pH}$ value that decreases

10 the efficiency of fET in the microcrystal suspension from $72 \%$ to $62 \%$. Most importantly,

11 however, we conclude that fET proceeds on the same timescale in crystals (320 ps) as in solution

12 (318 ps at $\mathrm{pH} 8.5,322 \mathrm{ps}$ at $\mathrm{pH} 5.5)$.

\section{S6. Quantum chemistry study of the $C v$ FAP reaction mechanism}

\section{6.1. Model of the CVFAP active site}

16 We computed a series of active-site models to analyze the chemical mechanism of the $C v$ FAP

17 photoenzyme. Starting from the coordinates of the high-resolution $100 \mathrm{~K}$ dark X-ray structure, 18 a model (272 atoms) containing a fatty acid (FA; $\left.\mathrm{C}_{10} \mathrm{H}_{21} \mathrm{CO}_{2}{ }^{-}\right)$, oxidized flavin, water molecules

19 Wat1 and Wat2 and conserved residues of the FAP active site (fig. S16) was prepared; its 20 geometry (1) was optimized using the R-B3LYP method. The structures of the diradical charge-

21 transfer (CT) intermediates (3) and (4), and intermediates/products (5), (5a), (5b), (6), and (6a) 22 formed after back electron transfer (bET) were obtained by gradually introducing the desired 
1 changes of the initial atomic coordinates to the optimized geometry (1) and subsequent geometry

2 optimization with the restricted (R-) or unrestricted (U-) B3LYP method depending on the

3 electronic state of interest. The energies and energy gradients of the optimized geometries are

4 listed in table S5.

The flavin demonstrates noticeable butterfly bending (in both oxidized and semiquinone

6 state) in all optimized active-site geometries, indicating that the intermolecular interactions

7 leading to bending are accounted for by our active site model. These interactions involve not

8 only the hydrogen bonds suggested by the distances in the high-resolution crystal structure, but

9 also by charge-transfer interactions between the flavin and protein, e.g. the electron-donating

10 polypeptide loop comprising A171-T172, as demonstrated by excitation energies and electronic

11 couplings (tables S6, S7).

12 6.2. Forward electron transfer (fET) is facilitated by rearrangement of Wat 1

13 Fig. 6 and fig. S22 illustrates hydrogen-bonding interactions of the fatty acid carboxylate in the 14 dark state (1) and the proposed rearrangement of Wat 1 (2). In the dark state (1), the carboxylate 15 forms multiple hydrogen bonds with surrounding residues and notably, with two water 16 molecules Wat1 and Wat2 resolved in the high-resolution crystal structures. Wat1 is positioned 17 between the flavin and R451, donating hydrogen bonds to the carbonyl backbone of S574 and 18 fatty acid carboxylate (O...O distance is 2.78 and $2.71 \AA$, respectively), and accepting a hydrogen 19 bond from R451 (O...N distance is $2.70 \AA)$. Wat2 accepts a hydrogen bond from Q486 (O...N

20 distance is $2.80 \AA$ ), and donates hydrogen bonds to the carbonyl backbone of S573 and 21 carboxylate (O...O distance is 2.84 and $2.67 \AA$, respectively). All distances are given for the 22 optimized structure (1). 
1 Attempts to optimize a geometry with Wat1 hydrogen-bonded with N5-flavin failed. Structure

2 (2) featuring the Wat1 - flavin N5 hydrogen bond undergoes rearrangement to structure (1)

3 during geometry optimization. The preferred interaction of Wat 1 is consistent with the negative

4 charges on the hydrogen-bond accepting atoms of the surrounding groups: $(-0.38)-\mathrm{O}$ of the

5 carboxylate, (-0.20) - N5-flavin, and (-0.31) - O of the S573 backbone carbonyl (the charges

6 are given for geometry (2) according to the Mulliken analysis of the B3LYP electron density).

7 The excitation spectrum in table S6 includes the excited states of the flavin and charge-

8 transfer (CT) states corresponding to electron transfer from the protein to the flavin. Among the

9 computed states at geometry (1), two transitions with high oscillator strengths predict two

10 absorption bands at 459 and $350 \mathrm{~nm}$ (computed energies 2.70 and $3.54 \mathrm{eV}$, respectively) in

11 agreement with the optical spectrum of oxidized FAD. The transition at $2.36 \mathrm{eV}$ corresponds to

12 a n $\pi^{*}$ excitation of the N5 lone pair of flavin with a weak oscillator strength. The transitions

13 indicated as charge transfer (CT) correspond to electron transfer from the protein to flavin,

14 namely from the side chains of Y466 and C432 as well as backbone and side chains of the A171-

15 T172 stretch. The CT transitions, as expected, have weak oscillator strengths.

Surprisingly, we did not find a state corresponding to electron abstraction from the fatty

17 acid carboxylate by flavin among the CT states computed at (1). All our attempts to obtain the

18 CASSCF solution with the carboxylate MOs contributing to the active space failed. Obviously,

19 multiple hydrogen bonds weaken the carboxylate electron-donor ability, in particular

20 interactions with Wat1 and Wat2. To demonstrate the effect of these water molecules, we

21 computed excited states for geometry (2) and additionally for geometry (1) from which we

22 excluded Wat2 (indicated as (1)-wo-Wat2 in table S6). Rearrangement of Wat1 in (2) favors

23 electron abstraction from the carboxylate because breakage of the hydrogen bond between Wat 1 
1 and carboxylate reduces the carboxylate negative charge stabilization whereas formation of a

2 hydrogen bond between Wat 1 and N5 stabilizes the negative charge of the flavin radical anion.

3 We obtained the CT fatty acid energy $2.8 \mathrm{eV}$ for model (1)-wo-Wat2 and $1.8 \mathrm{eV}$ for model (2).

4 We propose that rearrangement of Wat 1 is triggered by flavin photoexcitation. Indeed, a

5 charge-transfer interaction with the loop forming the flavin binding pocket (CT A171-T172 state

6 in table S6) may facilitate Wat1 rearrangement following flavin photoexcitation. A significant

7 electronic coupling between the excited flavin and A171-T172 (40 meV; table S7) indicates that

8 the photoexcited flavin is readily converted to the flavin radical anion that becomes stabilized

9 by a hydrogen bond from Wat1. This Wat1 rearrangement in (2) not only reduces the CT fatty

10 acid energy, but also increases the electronic coupling driving electron abstraction from the fatty

11 acid carboxylate to $47 \mathrm{meV}$ (table $\mathrm{S} 7$ ).

12 6.3. Fatty acid decarboxylation in the charge-transfer (CT) state

13 To characterize fatty acid decarboxylation triggered by electron abstraction, we performed 14 relaxed-scan energy calculations along the OCO bending coordinate for a reduced model of the 15 active site (fig. S18) consisting of the fatty acid radical, several side chains and Wat2. This model

16 was computed with the U-B3LYP method to obtain an estimate of the decarboxylation energy

17 barrier for the fatty acid radical in the FAP active site. The relaxed geometry scan for the OCO

18 bending coordinate indicates that $\mathrm{CO}_{2}$ dissociation from the fatty acid radical proceeds without

19 any energy barrier, whereas the energy of the alkyl radical and $\mathrm{CO}_{2}$ products is substantially

20 lower than the energy of the initial fatty acid radical. The dissociation is accompanied by rotation

21 of the $\mathrm{CO}_{2}$ group with respect to the alkyl chain. 
2 Formation of the alkyl and flavin radicals in the FAP active site was modeled starting from the

3 coordinates of the partially optimized geometry (2) containing Wat1 hydrogen bonded to flavin

4 N5. The OCO angle in the fatty acid was increased to 160 degrees and the geometry was

5 optimized in the triplet state (unpaired electrons on the alkyl and flavin radicals) using the U-

6 B3LYP method. Optimization led to $\mathrm{CO}_{2}$ dissociation. Changing the coordinates of the alkyl

7 radical in order to decrease the distance between the $\mathrm{CH}_{2}(\mathrm{rad})$ center and $\mathrm{Y} 466$ side chain (which

8 is suggested by the substantial electronic coupling of these radicals; $142 \mathrm{meV}$ in table S7)

9 followed by geometry optimization yielded structure (3). The flavin geometry remained bent in

10 the radical-anion state.The excited-state calculations (table S11) demonstrate that the diradical

11 CT state becomes the ground state of structure (3) whereas the closed-shell (CS) state,

12 corresponding to $\mathrm{bET}$, is more than $3 \mathrm{eV}$ above the CT diradical state. Two electronic transitions

$13(0-1)$ and (0-2) with high oscillator strengths predict the flavin radical absorption to be red-

14 shifted compared to the oxidized flavin which is consistent with spectroscopy data on FAP and

15 other flavoproteins.

\section{6.5. Pathways leading to alkane formation}

\section{6.5.1. HAT pathway involving Cys432}

18 Theoretically, the alkyl radical can be converted to the alkane by hydrogen atom transfer (HAT)

19 from Y466 or C432. For the possible HAT process, Y466 is ruled out by the experimental data

20 obtained for the Y466F mutant showing almost unperturbed electron-transfer kinetics in

21 comparison to the wild type. To address HAT from C432, we optimized the geometries of the

22 diradical intermediate, resulting from the HAT reaction (4) using the UB3YP method. At the 
1 optimized geometry (4), flavin re-oxidation was modelled by switching the electronic state from

2 the diradical to the closed shell (CS), continuing geometry optimization with the R-B3LYP

3 method. Obtained intermediate (5) contains oxidized flavin and deprotonated thiol C432.

4 Intermediate (5a) was obtained by proton transfer (PT) along the hydrogen bond of R451 with

5 C432 in (5) and R-B3LYP geometry optimization. Reaction (3) $\rightarrow$ (4) is energetically favorable

6 (energy decrease by $15 \mathrm{kcal} / \mathrm{mol}$; table S5), and it has a $8 \mathrm{kcal} / \mathrm{mol}$ energy barrier (estimated

7 using a truncated model; figure S18). Much less energetically favorable is subsequent bET (4)

$8 \rightarrow(5)$ (only $3 \mathrm{kcal} / \mathrm{mol}$ energy reduction; table S5). The excitation energies in table $\mathrm{S} 11$ suggest

9 that flavin-radical absorption band might blue-shift in intermediate (4) in comparison to

10 intermediate (3) (2.67 and $2.44 \mathrm{eV}$ ) whereas the CS energy (corresponding to bET) is lower in

11 (4) than in (3) (0.83 and $3.14 \mathrm{eV})$. Electronic coupling driving bET from flavin to C432 radicals

12 is only $4 \mathrm{meV}$ (table S7), indicating that flavin re-oxidation by C432 might be limited by

13 electronic coupling.

14 6.5.2. PCET pathways involving Wat1, Wat2, and Arg451

15 We modelled bET for geometry (3) by continuing its geometry optimization in the closed-shell

16 state with R-B3LYP. The optimization demonstrated an increase of interactions between Wat2

17 and alkane anion suggesting protonation of the alkane in concert with bET. This protonation

18 yielded alkane and $\mathrm{OH}^{-}$anion that obtained a proton from $\mathrm{R} 451$ recovering Wat2 in geometry

19 (5) (fig. S19). The energy of (5) is $27 \mathrm{kcal} / \mathrm{mol}$ higher than the energy of (1) (table S5) due to

20 the presence of deprotonated R451, and hence, it is conceivable that (5) undergoes further

21 stabilization (fig. S19). The energy decreases when Wat1 forms a hydrogen bond with R451

22 instead of Wat2. Further substantial stabilization by about $15 \mathrm{kcal} / \mathrm{mol}$ involves R451

23 protonation by Wat 1 and bicarbonate formation from the hydroxyl anion $\mathrm{OH}^{-}$derived from Wat 1 
1 and $\mathrm{CO}_{2}$ (6). The energy barrier for (5) $\rightarrow$ (6) was estimated by relaxed energy scan calculations

2 (fig. S19). The energy barrier for bicarbonate formation facilitated by re-protonation of R451 is

3 about $4 \mathrm{kcal} / \mathrm{mol}$. Bicarbonate formation might involve Wat 2 instead of Wat1: the hydroxyl

4 anion $\mathrm{OH}^{-}$derived from Wat2 directly binds $\mathrm{CO}_{2}(5 b)$. The bicarbonate involving Wat2 is 10

$5 \mathrm{kcal} / \mathrm{mol}$ less energetically favorable than formation involving Wat 1.

6 The reaction scheme (Fig. 6C) highlights specific roles of Wat1 and Wat2 in the 7 proposed catalytic mechanism. Alkane formation involves Wat2 and R451 as primary and

8 secondary proton donors, respectively. However, deprotonated arginine is a high-energy species,

9 and its reprotonation by either Wat 1 or Wat 2 followed by bicarbonate formation is energetically

10 favorable. Irrespective of the origin, the bicarbonate is rather stable with respect to back

11 conversion to $\mathrm{CO}_{2}$, since protonated $\mathrm{R} 451$ is a rather poor acid for bicarbonate destabilization

12 (fig. S19). An alternative stronger acid cannot be suggested by our present computations.

Thus, our calculations starting from high-resolution crystal structures suggest that FAP

14 needs two waters, Wat1 and Wat2, interacting with the substrate at the active site for its

15 functional mechanism. The distinct chemical roles of these waters are defined by their

16 interactions at the active site. Wat 1 facilitates fET by stabilizing the flavin radical anion whereas

17 Wat 2 serves as a primary proton donor during the alkane formation. In addition, both Wat 1 and

18 Wat2 may participate in the formation of the bicarbonate byproduct by interacting with R451

19 hence facilitating the unusual for arginine proton-donor function. According to our current active

20 site model, Wat 1 is slightly more preferred than Wat 2 for bicarbonate formation. 
2 Table S8 presents computed excitation spectra of species (5), (5a), (5b) and (6) that are

3 candidates for the spectroscopically identified red-shifted flavin state $F_{A D}$. The flavin

4 excitation energy decreases for all candidates compared to the dark state (1). The spectral shift

5 results from moving the anionic charge away from the flavin (geometries (5), (6), and (6a)) or

6 from charge neutralization and Wat1 hydrogen bonding to flavin N5 (geometry (5a). The flavin

7 butterfly bending angle and distances from the anionic or neutral $\mathrm{CO}_{2}$ fragment to flavin $\mathrm{N} 5$ in

8 the FAD ${ }_{\text {RS }}$ candidate structures are shown in fig. S20.

\section{6.7. Effects of the C432S mutation on $f E T$}

10 To address the effect of the C432S mutation, a model of the active site (275 atoms) was prepared,

11 in a similar way as the WT model, from the coordinates of the C432S X-ray structure. The

12 calculations were performed for structures $\left(1^{\mathrm{C} / \mathrm{S}}\right)$ and $\left(2^{\mathrm{C} / \mathrm{S}}\right)$ that are analogous to structures $(1)$

13 and (2) of WT. Interaction with an additional water molecule (Wat3; fig. S22) further reduce the

14 ability of the bicarbonate to donate an electron to excited flavin. By computing excitation

15 energies (table S10), we demonstrate the effect of water molecules Wat 2 and Wat 3 on the CT

16 energy. Stabilization of the carboxylate with Wat3 increases the energy of the CT fatty acid by

$170.2 \mathrm{eV}$ in comparison to WT. The CT A171-T172 energy also increases in C432S. At the same

18 time, the excited flavin energy does not change in the mutant model in comparison to WT. This

19 energy charge (decrease of the $\mathrm{S}_{1}$ energy in comparison to the CT energies) suggests a decrease

20 of the driving force for fET. The electronic coupling of flavin with the A171-T172 loop

21 controlling Wat1 rearrangement and facilitating fET decreases in the mutant in comparison to

22 WT (22 and $40 \mathrm{meV}$, respectively; Table S7), and at the same time, after rearrangement of Wat1 
1 the coupling to the fatty acid remains moderate ( $8 \mathrm{meV}$ in mutant and $47 \mathrm{meV}$ in WT; table S7).

2 Accordingly, fET in the mutant is limited by the weak interactions with the substrate, whereas

3 wt fET is determined by flavin coupling to A171-T172. Using the semiclassical formula of

4 Marcus theory, the five time smaller electronic coupling (40 meV in WT and $8 \mathrm{eV}$ in $\mathrm{C} 432 \mathrm{~S}$ )

5 together with the decrease of the driving force by $0.2 \mathrm{eV}$ reduce the ET rate by a factor of 175

6 (assuming $1 \mathrm{eV}$ reorganization energy). This estimate is in quantitative agreement with the

7 experimentally observed reduction of the fET rate in the mutated protein. Hence, the

8 computational analysis demonstrates that structural differences observed in the WT and mutated

9 proteins are consistent with changes of $\mathrm{fET}$ in these proteins determined by transient 10 spectroscopy experiments.

\section{6.8. Excitation and redox energies of bent flavin}

12 Our protein crystallography analysis demonstrates that the isoalloxazine ring of the flavin

13 chromophore adapts a butterfly bent configuration in the dark state of $C v \mathrm{FAP}$, which is a

14 manifestation of the steric and electronic interactions of the flavin with the surrounding protein.

15 Previous computational results suggest that in general, flavin bending facilitates flavin reduction

16 (36). In the case of FAP this indicates that bending stabilizes flavin radical anion against bET.

Table S9 presents our computational results addressing the effect of bending on

18 photoinduced fET. To study the effect of bending qualitatively, we modeled the geometric effect

19 of the steric interactions in a simplified flavin model fixing the torsions around the $\mathrm{C}-\mathrm{N}$ bonds

20 of the middle ring to 160 or 150 degree during geometry optimization (corresponding to butterfly

21 bending of 20 or 30 degree, respectively). This simple model does not account for electronic

22 interactions between the flavin and protein, which contribute in addition to the steric constraints. 
1 To compensate for this limitation, we considered an active site (AS) model derived in the

2 oxidized and radical state derived from geometries (1) and (3), respectively. Despite smaller 15-

3 degree bending, the energy changes in the AS model (with respect to the panar flavin) are

4 substantially larger in comparison to those in the simplified model with the 20- and 30- degree

5 bending angle, suggesting that electronic interactions omitted in the simplified model

6 significantly enhance the effect of the restrained torsion.

7 Figure S21 demonstrates changes of the flavin excitation energy, and vertical and

8 adiabatic electron affinity. Notably, bending significantly decreases the oxidized-flavin

9 excitation energy. Even to a larger extent, bending diminishes the vertical electron affinity which

10 defines the CT energies in the flavin excitation spectrum. Hence, bending stabilizes the CT states

11 with respect to the excited flavin facilitating fET.

\section{6.9. Comparison of computational and experimental results}

13 Computational results addressed the most prominent features of the FAP active site that were

14 revealed by the high-resolution protein structures. These features include the oxidized flavin

15 demonstrating a bent conformation of the isoalloxazine system, two water molecules interacting

16 with the substrate and the R451 counterion of the fatty acid substrate and changes of the fatty-

17 acid carboxyl interactions in the mutated proteins. The molecular interactions associated with

18 these features were linked by the spectroscopic observations: the red-shifted UV-Vis absorption

19 of the flavin chromophore, flavin fluorescence signal decay, changes of the flavin absorption

20 accompanying the catalytic cycle, formation of $\mathrm{CO}_{2}$ on the sub nanosecond time scale, back

21 electron transfer coupled to proton transfer (PCET) suggested by the kinetic isotope effect. 
1 Moreover, the calculations suggest a possible mechanism for bicarbonate formation and arginine

2 deprotonation, which would be consistent with the FTIR spectral changes.

In particular, the computations predict bicarbonate formation involving either Wat 1 or

4 Wat2 that may follow splitting of Wat2 coupled to bET. In both identified PCET pathways (II

5 and III in Fig.6), the hydroxyl derived from Wat2 is transiently formed at the bET step. This

$6 \mathrm{OH}^{-}$either deprotonates $\mathrm{R} 451$ or interacts with $\mathrm{CO}_{2}$ yielding a bicarbonate. Such a partitioning

7 reaction presumably leading to formation of the red-shifted species would be in line with the

8 concomitant disappearance of the $\mathrm{CO}_{2}$ signal with formation of the red-shifted species observed

9 by time-resolved IR (Fig. 2). In contrast, the reaction involving Wat1 to form bicarbonate is

10 likely a sequential process following bET, and thus, it occurs in the flavin red-shifted state. The

11 reaction involves a re-protonation of R451 by Wat1 leading to bicarbonate formation. In order

12 to be consistent with the spectroscopic data, bicarbonate formation involving Wat 1 would have

13 to occur on a similar time-scale as the disappearance of the $\mathrm{CO}_{2}$ signal and formation of the red-

14 shifted species, which implies a very short lifetime of the deprotonated R451. Consistent with a

15 rather small energy barrier stabilizing the deprotonated R451 state, the FTIR signal assigned to

16 arginine deprotonation was observed only at $100 \mathrm{~K}$ but not at $150 \mathrm{~K}$.

While the sites of bicarbonate formation differ slightly for the partitioning reaction and

18 sequential reaction, they are both close to the flavin and R451. In particular, hydrogen bonding

19 with the protonated R451 is expected to stabilize the newly formed bicarbonate in either case. It

20 is thus rather surprising that no electron density features consistent with bicarbonate were

21 observed close to FAD and R451 in the cryocrystallography (Fig. 4D) or TR-SFX (Fig. 5A) data

22 of illuminated crystals. Instead, electron density consistent with bicarbonate was observed very

23 close to C432. This would imply that bicarbonate diffused there shortly after its formation, but 
1 the driving force for this process is unclear. Alternatively, bicarbonate might actually be formed

2 close to $\mathrm{C} 432$ which would suggest that $\mathrm{C} 432$ has a catalytic role, e.g. involved in a hydrogen

3 atom transfer (HAT). In this case, the formed C432 radical would then be reduced by bET from

$4 \mathrm{FAD}^{\bullet-}$, resulting in a red-shifted oxidized flavin $\mathrm{FAD}_{\mathrm{RS}}$ in $\sim 100 \mathrm{~ns}$. However, to be consistent

5 with the absence of cysteine deprotonation in the $\mathrm{FAD}_{\mathrm{RS}}$ form as assessed by cryotrapping FTIR

6 (Fig. 4A), this bET would have to be coupled to protonation of the C432 anion (within the $~ 100$

7 ns phase). Furthermore, the acceleration of bET/red shift formation in the R451K mutant

8 occurring in spite of the unfavorable orientation of the C432 side chain for HAT to the alkyl

9 radical and bET from $\mathrm{FAD}^{\bullet-}$ in this mutant, would argue against a catalytic role of $\mathrm{C} 432$ in the

10 FAP photocycle.

11 The fate of the bicarbonate pool and possible transformation of bicarbonate back to $\mathrm{CO}_{2}$.

12 remains unclear. Transformation of $\mathrm{CO}_{2}$ to $\mathrm{HCO}_{3}{ }^{-}$involving either Wat 1 or Wat2 lowers the

13 energy. Therefore, reverse reactions - proton transfer from R451 to bicarbonate to recover Wat1

14 or Wat2 and $\mathrm{CO}_{2}$ - are ruled out because of their unfavorable energy. If $\mathrm{HCO}_{3}{ }^{-}$escapes from

15 the protein as such, it should not turn into $\mathrm{CO}_{2}$ in solution given the experimental $\mathrm{pH}$ of 8.2 (and

16 certainly not on the microsecond/millisecond time scale). Back transformation of $\mathrm{HCO}_{3}{ }^{-}$to $\mathrm{H}_{2} \mathrm{O}$

$17+\mathrm{CO}_{2}$ is hence more likely initiated within the protein by a residue other than $\mathrm{R} 451$. This process

18 is subject to future investigations. 
A

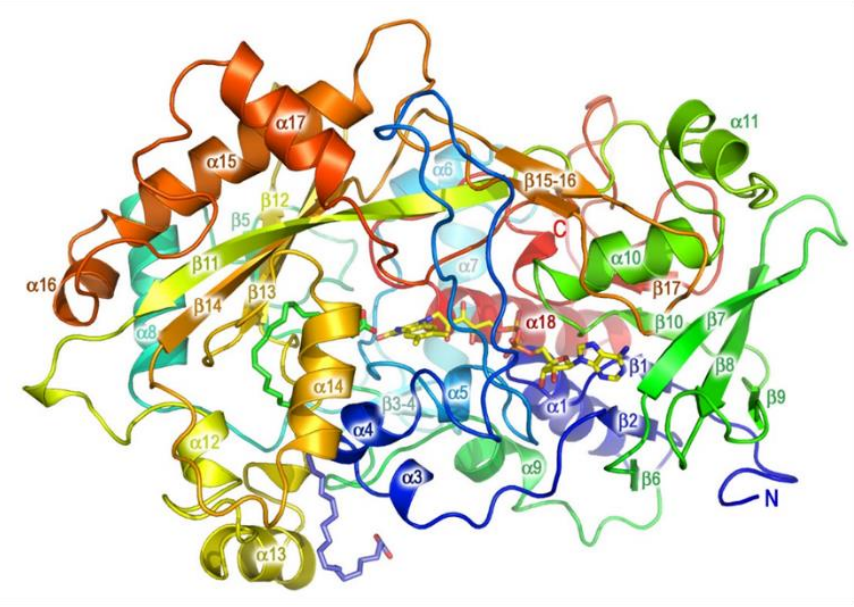

B

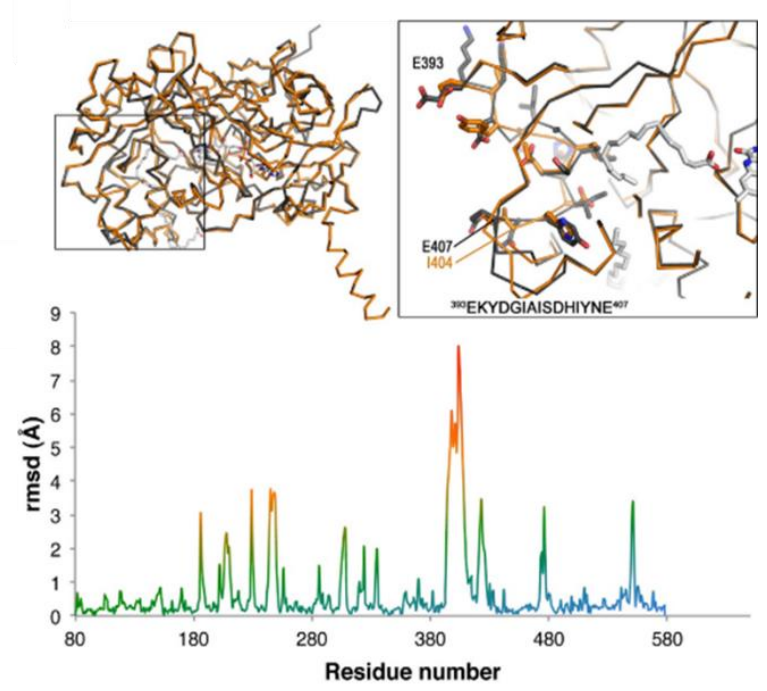

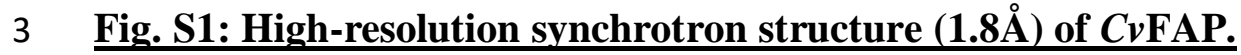

4 (A) Overall structure of Chlorella variabilis FAP ( $C v \mathrm{FAP})$ in the dark (' $100 \mathrm{~K}$ dark') showing

5 the numbered secondary structure elements. (B) Structural comparison between medium- (4)

6 and high-resolution structures of $C \nu \mathrm{FAP}$ (present study). Upper panel: the medium-resolution

7 structure (featuring an N-terminal tail, residues 61 to 76) is shown in gold and the high-resolution

8 one (no N-terminal tail) is shown in silver. Inset: magnification of the region that appeared to be

9 poorly structured in the medium-resolution structure (residues 392 to 407). Lower panel: average

10 distance between the $\mathrm{Ca}$ atoms of the two superimposed structures as shown by the root-mean-

11 square deviation of atomic positions. 
A<smiles>[R]n1c2nc(=O)[nH]c(=O)c-2nc2cc(C)c(C)cc21</smiles>

B

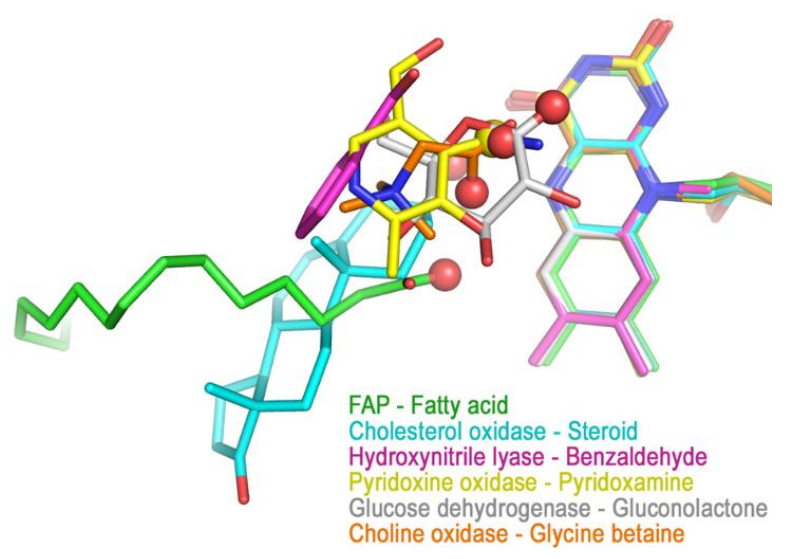

2 Fig. S2: Isoalloxazine ring of FAD.

3 (A) Numbering of the atoms in the isoalloxazine ring of FAD. (B) Comparison of substrate or

4 product position relative to FAD isoalloxazine ring in GMC oxidoreductase family of enzymes.

5 FAD cofactors are shown as sticks on the right . Substrates or products are also shown as sticks,

6 with their closest atom involved in the catalytic reaction shown as a sphere. Except for FAP, all

7 the major GMC stabilize their substrate close to the central N5 atom of FAD. PDB used are

8 1COY (Cholesterol oxidase), 3GDN (Hydroxynitrile lyase), 4HA6 (Pyridoxine oxidase), 4YNU

9 (Glucose dehydrogenase) and 4MJW (Choline oxidase). The shortest distance between the

10 carboxyl group and the benzene ring plane of the flavin is $3.1 \AA$ and the angle between them is

11 below $35^{\circ}$, indicative of an anion-quadrupole interaction (118). To our knowledge, it is the first

12 clear structural characterization of such a feature in enzyme substrate interaction, with two other

13 putative examples in chloride transport $(119,120)$. 
A

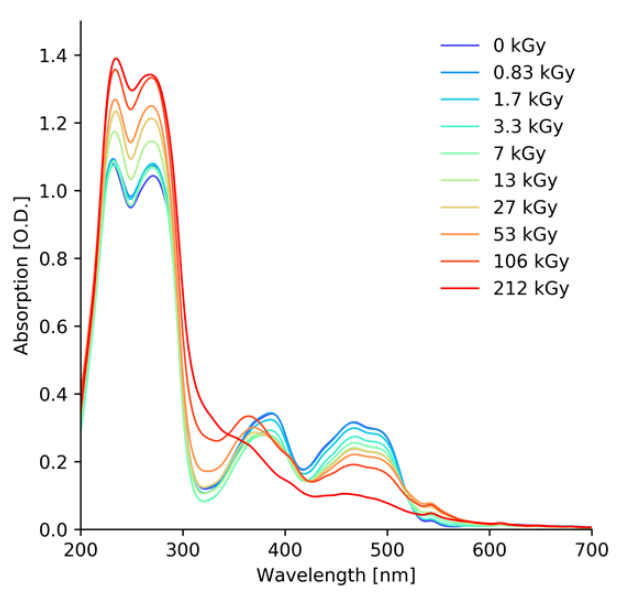

B

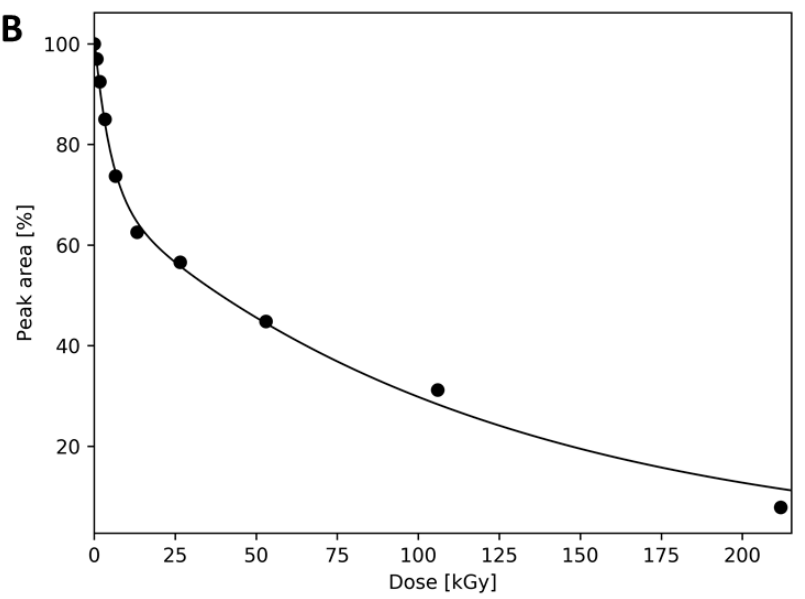

2 Fig. S3: X-ray dose-resolved UV-Vis absorption spectra of a $C v F A P$ crystal at room

3 temperature.

4 (A) Series of UV-Vis absorption spectra recorded at room temperature on a FAP crystal with

5 increasing X-ray doses showing the progressive reduction of the FAD. (B) Decay curve of the

6 area of the FAD absorption peak between 410 and $540 \mathrm{~nm}$ (black circles) and an exponential fit

7 (black curve) indicating a reduction half-dose of $\sim 40 \mathrm{kGy}$.

8

9

10

11

12 
A

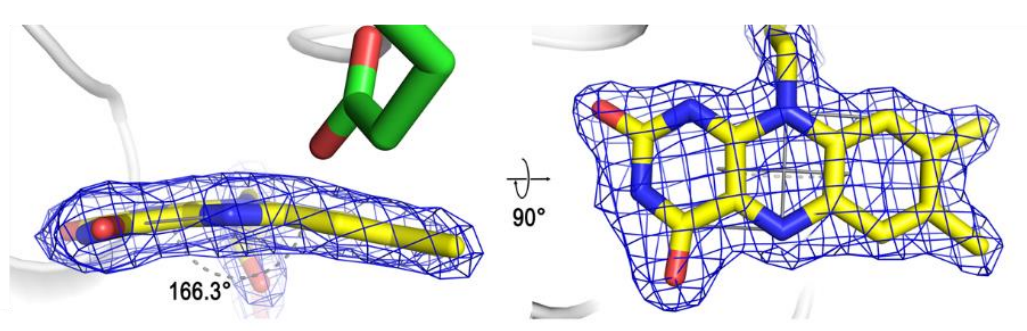

B
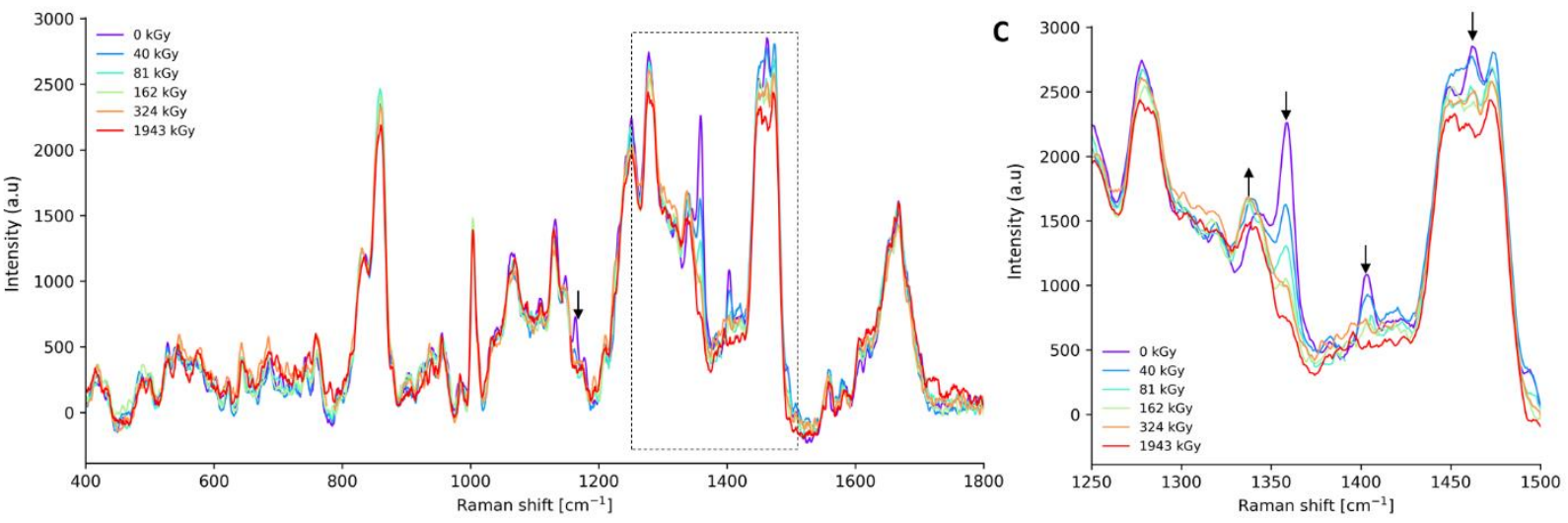

2 Fig. S4: Low dose structure at room temperature and X-ray dose-resolved Raman spectra

3 series of a $C v$ FAP crystal at $100 \mathrm{~K}$.

4 (A) 'RT dark low-dose' FAP structure depicting a bent FAD. The dihedral angle (C4-N5-N10-

5 C9) is indicated and the $2 F_{\mathrm{o}}-F_{\mathrm{c}}$ map is contoured at $2.5 \sigma$. (B) Series of non-resonant Raman

6 spectra recorded at $100 \mathrm{~K}$ on a FAP crystal cryoprotected with $20 \%$ glycerol with increasing X-

7 ray doses. (C) Close-up on the region showing the decrease, or increase of FAD Raman modes

8 due to X-ray induced reduction. The spectra do not show the global collapse of peak intensity

9 for the Raman modes of FAD that is expected for the X-ray induced butterfly movement (26),

10 and only a few stretching modes are affected (indicated by arrows), which can be explained by

11 the change in redox state of FAD without a significant structural change. 
A

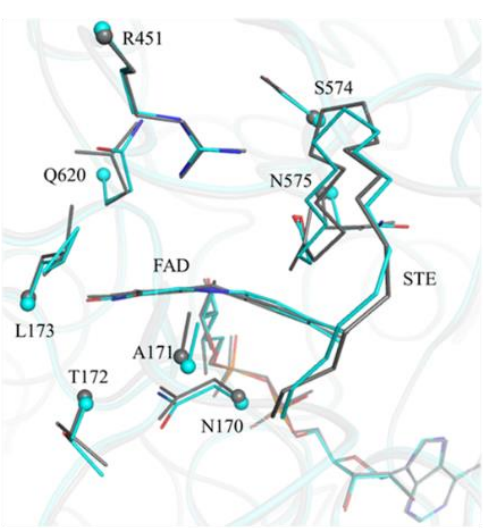

B

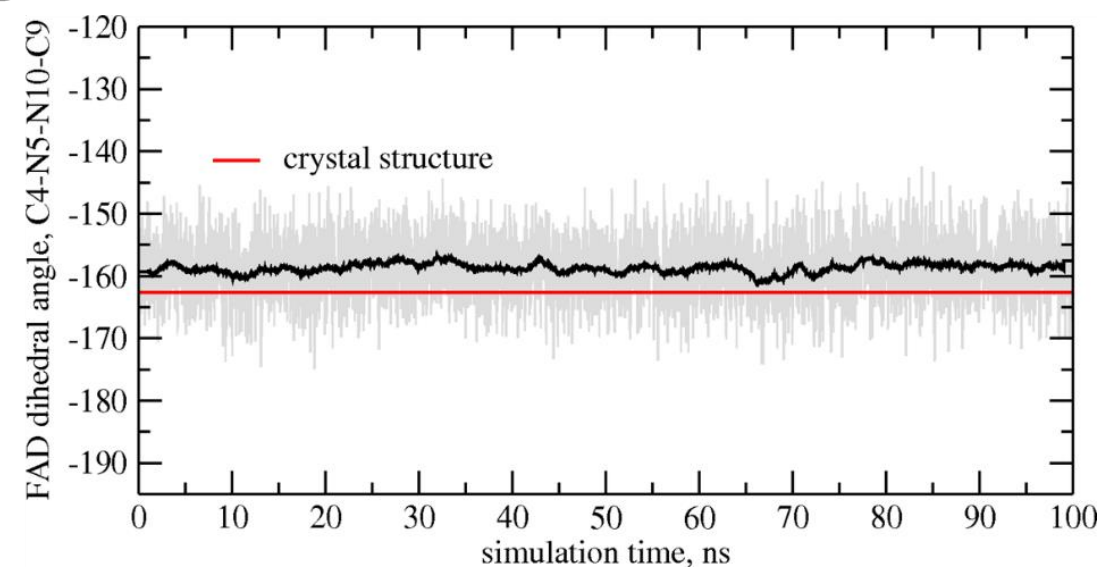

1

2 Fig. S5: Flavin dihedral angle observed in Molecular Dynamics simulations.

3 (A) Superposition of the crystal structure (synchrotron dark structure) around FAD and an

4 average structure observed in Molecular Dynamics (MD) simulations. The experimental

5 structure is shown in gray and the averaged MD structure is in color. The bent conformation of

6 flavin is clearly visible. (B) The black line shows average fluctuations of the dihedral angle C4-

$7 \quad$ N5-N10-C9 of the oxidized FAD observed in MD simulations and the black line shows the

8 dihedral angle of the oxidized FAD in the crystal structure (synchrotron dark structure). 


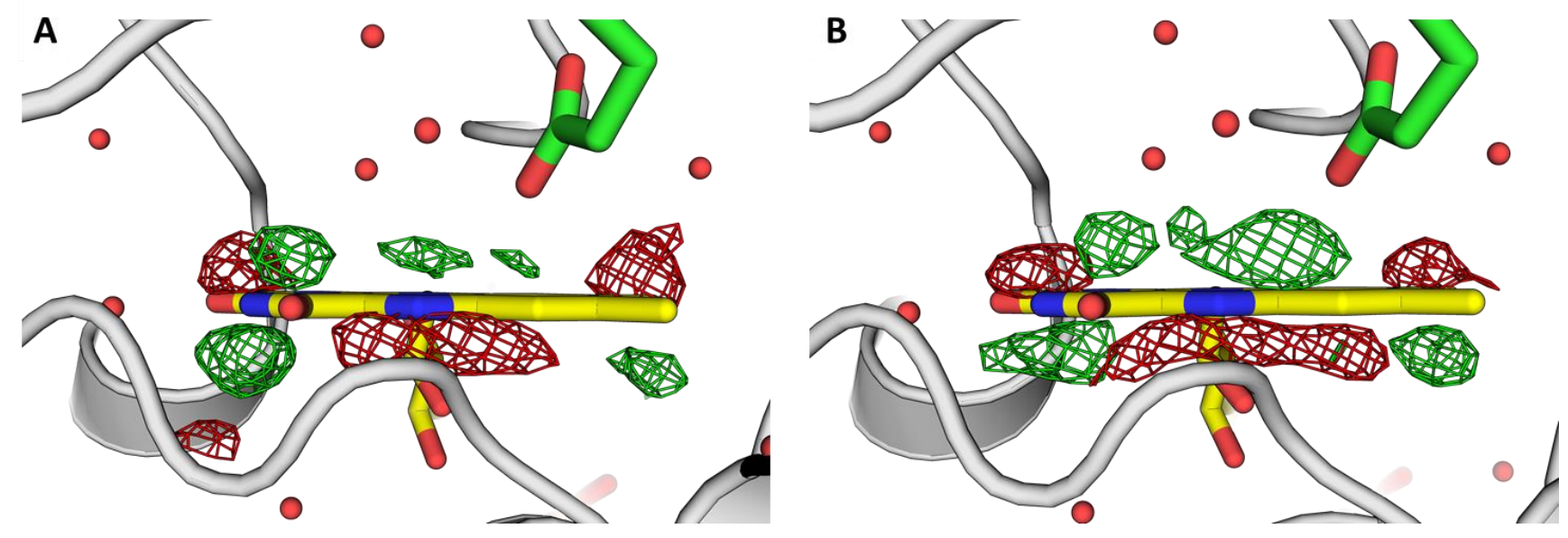

2 Fig. S6: Residual $m F_{\text {obs }}-D F_{\text {calc }}$ peaks indicate isoalloxazine rings of the FAD cofactor are

3 not planar in the SFX dark-state structure.

4 When the isoalloxazine ring of the FAD cofactor (yellow stick model) are restrained to be planar

5 in the SFX structure $\left((\boldsymbol{A})\right.$ molecule $\mathrm{A},(\boldsymbol{B})$ molecule B), peaks in the $m F_{\text {obs }}-D F_{\text {calc }}$ map $(+3 \sigma$

6 and $-3 \sigma$ in green and red, respectively) indicate bending. The fatty acid is shown in green, the

7 protein moiety in grey.

8 

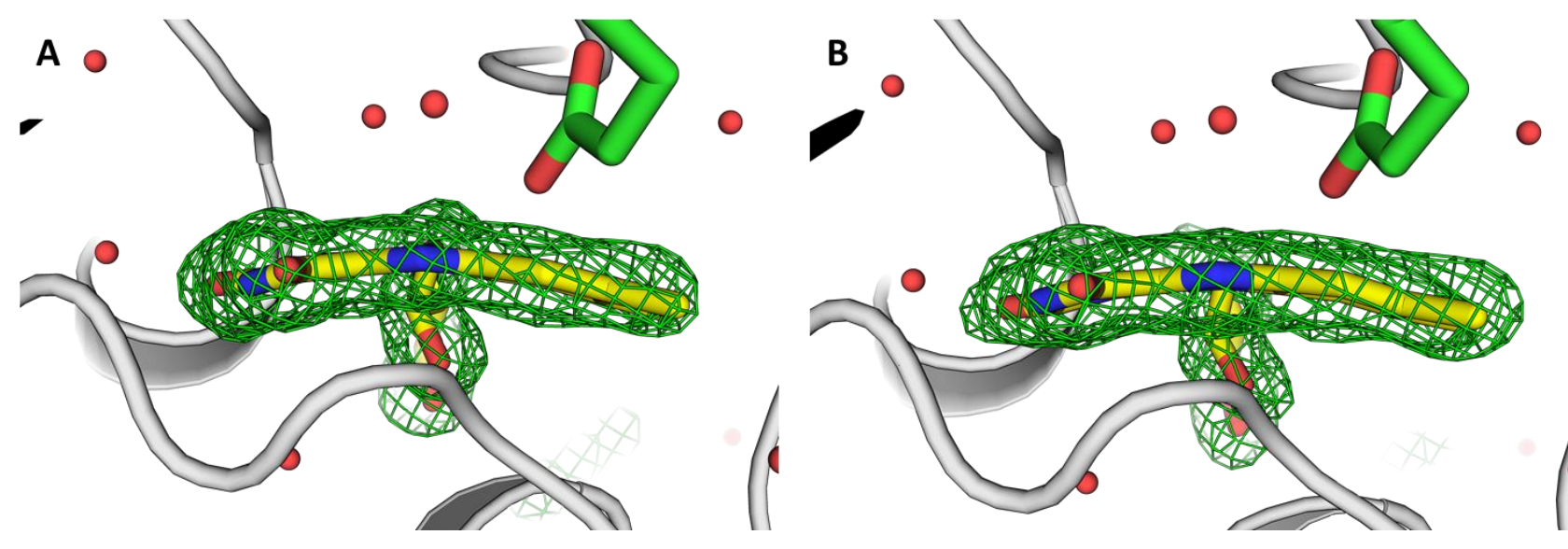

$2 \quad$ Fig. S7: FAD bending in the SFX dark-state structure.

3 The $m F_{\text {obs }}-D F_{\text {calc }}$ omit map ( $3 \sigma$, green) indicates bending of the isoalloxazine ring of the FAD

4 cofactor (dark-blue stick model) in molecules $\mathrm{A}(\boldsymbol{A})$ and $\mathrm{B}(\boldsymbol{B})$. The bending angle is $14.3^{\circ}$ and

$5 \quad 11.7^{\circ}$ in molecule $\mathrm{A}$ and $\mathrm{B}$, respectively. The fatty acid is shown in green, the protein moiety in 6 grey. 

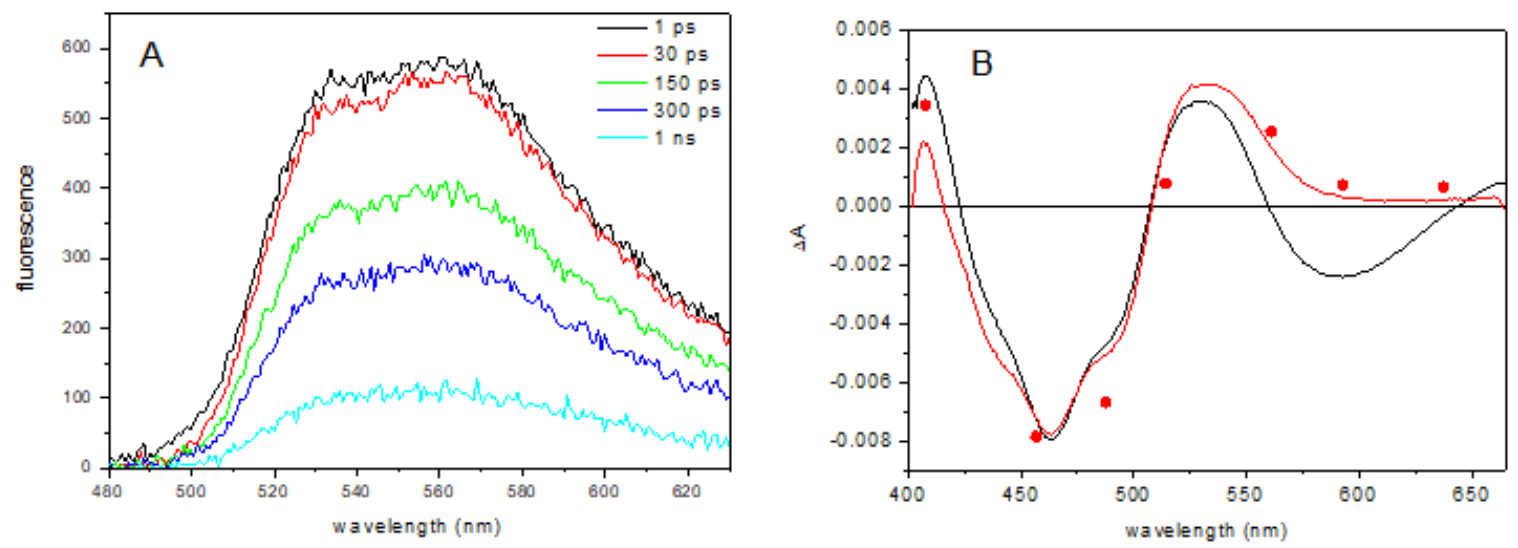

2 Fig. S8: Ultrafast fluorescence and visible absorption spectroscopy on $C v F A P$ in solution

3 in the presence of substrate.

4 (A) Fluorescence spectra at different delay times, highlighting dominant decay on the 300-ps

5 timescale. The corresponding kinetics are shown in Fig. 2A. Upon long exposure of the sample

6 to light and higher excitation densities the decay slows down (not shown), reflecting substrate

7 depletion. (B) Global analysis of the transient absorption data corresponding to Fig. 2B in terms

8 of a 350 ps phase (close to the time constant found for fluorescence decay rise of the $\mathrm{CO}_{2}$ signal

9 in the mid-IR) and a long-lived phase. The spectra associated with the initial state (black line,

$10 \mathrm{FAD}^{*}-\mathrm{FAD}_{\mathrm{Ox}}$ ) and the final state (red line) emerging from the analysis are shown. The

11 spectrum of the final state is in close agreement with the initial spectrum (red points, $10 \mathrm{~ns}$ ) from

12 single flash absorption experiments (4) assigned to $\mathrm{FAD}^{\bullet-}-\mathrm{FAD}_{\mathrm{OX}}$. As no further phases were

13 observed, this implies that electron transfer from the substrate to FAD occurs concomitant with

14 FAD* decay in $\sim 300$ ps. In passing we mention that the present full spectral characterization

15 allows to assess a significant difference in the $500-550 \mathrm{~nm}$ region in the final spectrum with a

16 model spectrum based on the $\mathrm{FAD}^{\bullet-}$ spectrum of glucose oxidase (4) suggesting that $\mathrm{FAD}^{\bullet-}$

17 absorbs stronger in the red part of the spectrum in FAP. 
$\triangle \mathrm{A}(\mathrm{mOD})$

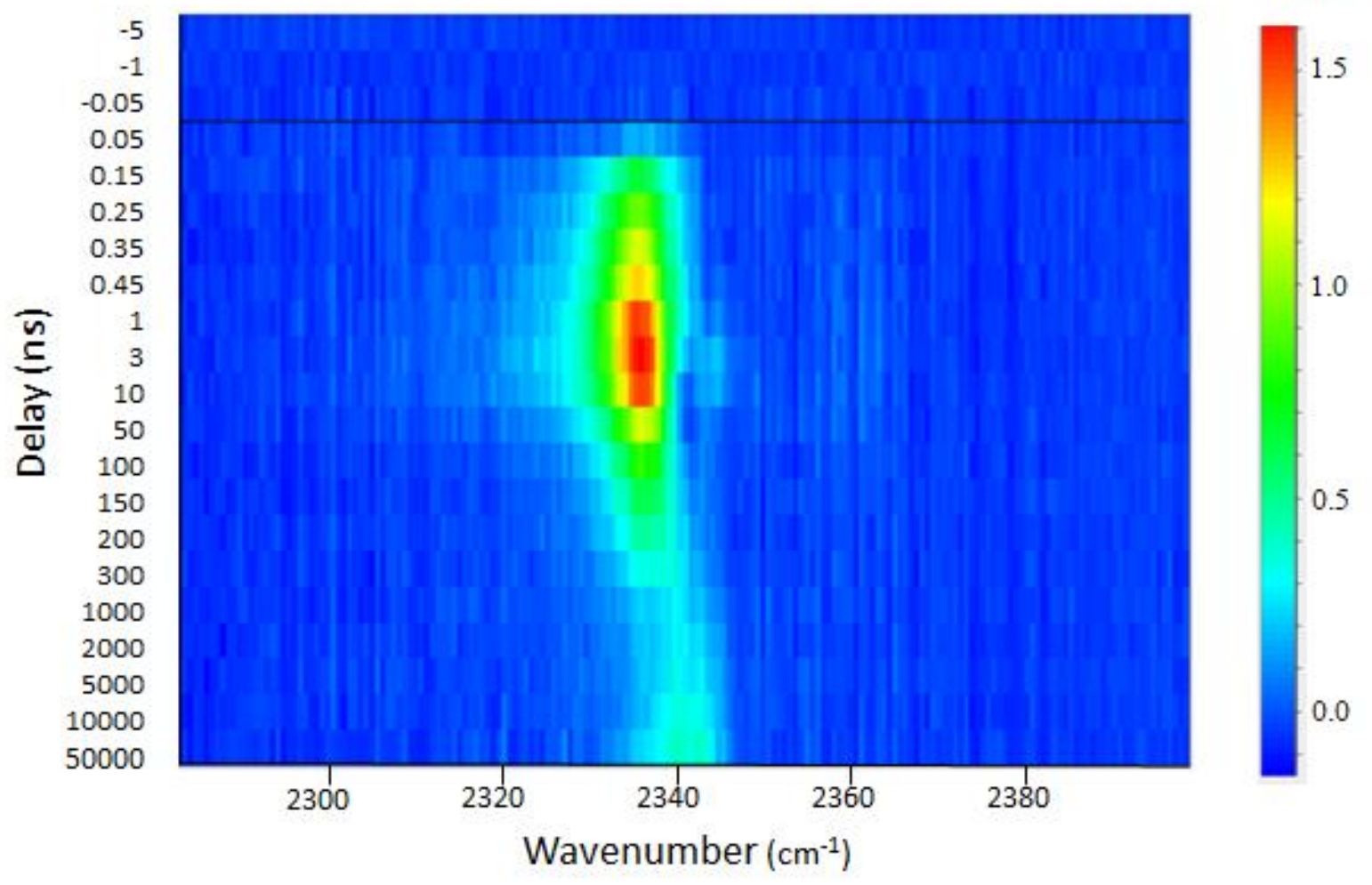

1

2 Fig. S9: Formation of $\mathrm{CO}_{2}$ co-product studied by TR-IR.

3 False-color representation of time-resolved IR data corresponding to Fig. 2C highlights shifting

4 to the value of $\mathrm{CO}_{2}$ in solution in a few $\mu$ s.

5

6

7

8

9

10 

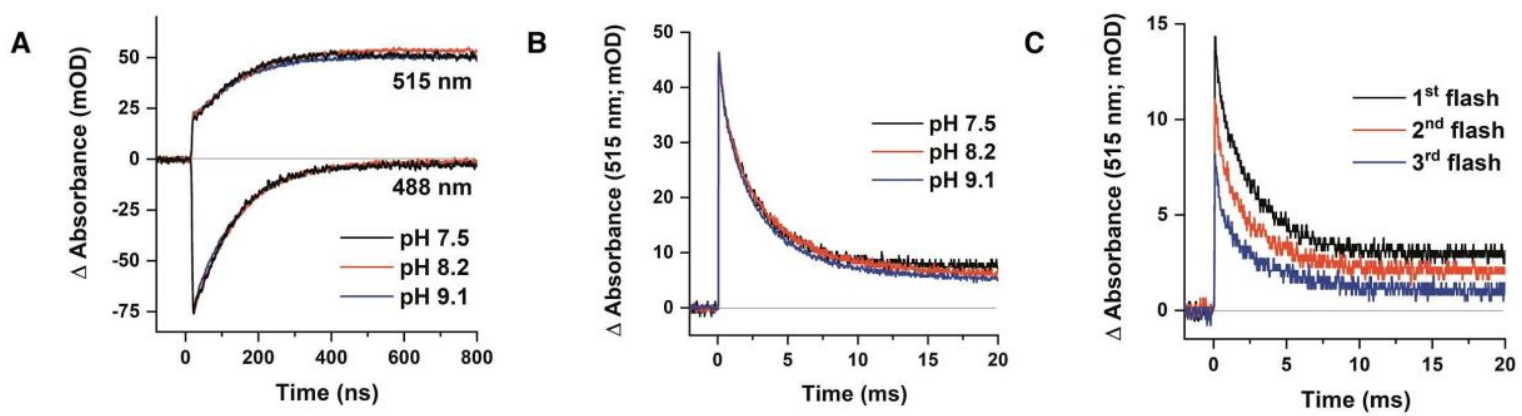

2 Fig. S10: Effects of $\mathrm{pH}$ and substrate consumption on the transient FAD absorption

3 changes in WT CvFAP.

4 (A) FAD transient absorption changes on the sub-microsecond timescale at different $\mathrm{pH}$ values.

5 Signals at $488 \mathrm{~nm}$ shows absorption bleaching due to FAD reduction to $\mathrm{FAD}^{\bullet-}$, followed by its

6 back reoxidation; signals at $515 \mathrm{~nm}$ reflect formation of the red-shifted reoxidized FAD

$7 \quad\left(\mathrm{FAD}_{\mathrm{RS}}\right)$. (B) Reversion of the transient FAD red shift at different $\mathrm{pH}$ values monitored at 515

$8 \mathrm{~nm}$ on the millisecond timescale. (C) Effect of the native substrate(s) consumption (by saturating

9 laser flashes at $470 \mathrm{~nm}$ ) on the disappearance of the transient FAD red shift monitored at 515

$10 \mathrm{~nm}$. 


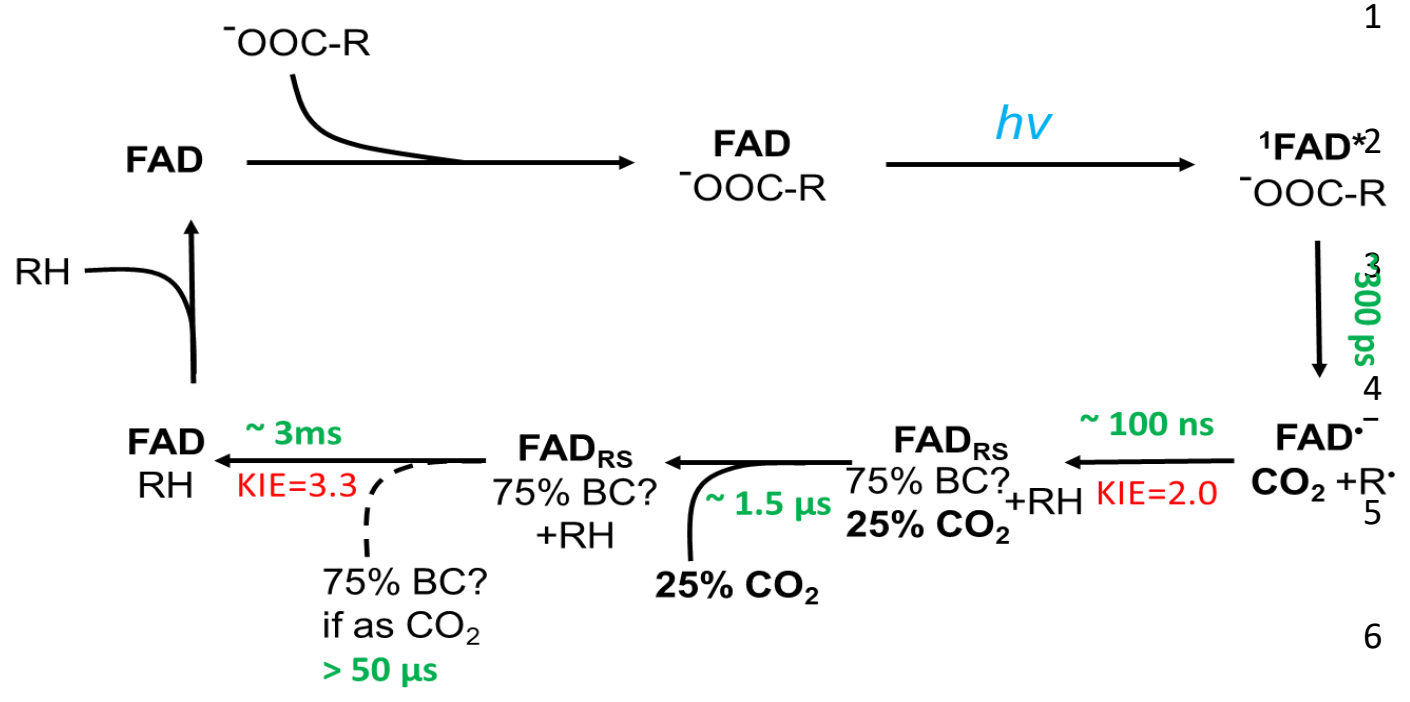

7 Fig. S11: Summary of the results obtained on $C v$ FAP using time-resolved infrared and UV-

$8 \quad$ Visible spectroscopies.

9 Spectroscopy shows that light-induced formation of a flavin radical $\mathrm{FAD}^{\bullet-}$ occurs with a time

10 constant $\tau \sim 300 \mathrm{ps}$, concomitant with decarboxylation of the deprotonated fatty acid substrate

11 OOC-R. Back electron transfer occurs with $\tau \sim 100 \mathrm{~ns}$, resulting in FAD $\mathrm{RS}_{\text {, reoxidized FAD }}$

12 displaying a transiently red-shifted absorption spectrum. The pronounced kinetic isotope effect

$13(K I E \approx 2)$ of this process suggests that it is coupled to a proton or hydrogen atom transfer to the

14 alkyl radical. On the same time scale, a major fraction of the formed $\mathrm{CO}_{2}$ is transformed to a

15 species believed to be bicarbonate (BC), which is supported by complementing cryotrapping

16 FTIR spectroscopy and possibly X-ray crystallography. During the subsequent process $(\tau \sim 1.5$

$17 \mu \mathrm{s}$ ) the remaining $\mathrm{CO}_{2}$ changes towards an environment spectroscopically identical to aqueous

18 solution. The TR-IR $\mathrm{CO}_{2}$ signal lost during the $\sim 100$ ns may recover on a timescale beyond 50

$19 \mu \mathrm{s}$, the time window of the experiment. $\mathrm{FAD}_{\mathrm{RS}}$ decays to the initial state with $\tau \sim 3 \mathrm{~ms}$, the

20 second process in the photocycle displaying an isotope effect $(K I E \approx 3)$. Species in bold were

21 directly observed by time-resolved spectroscopy. 


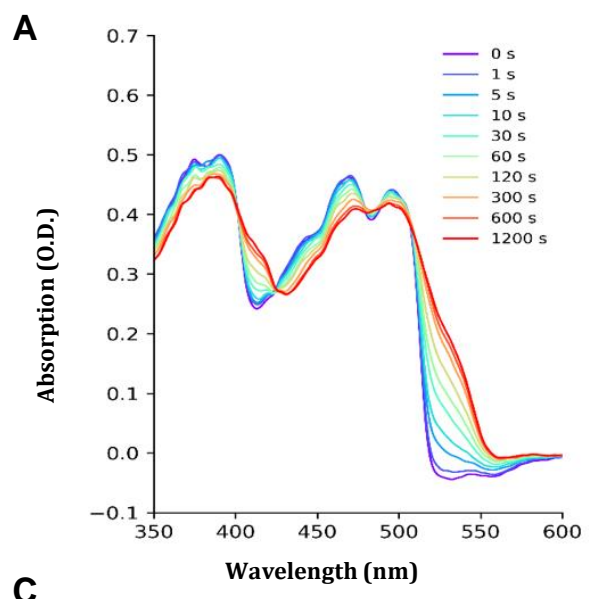

B
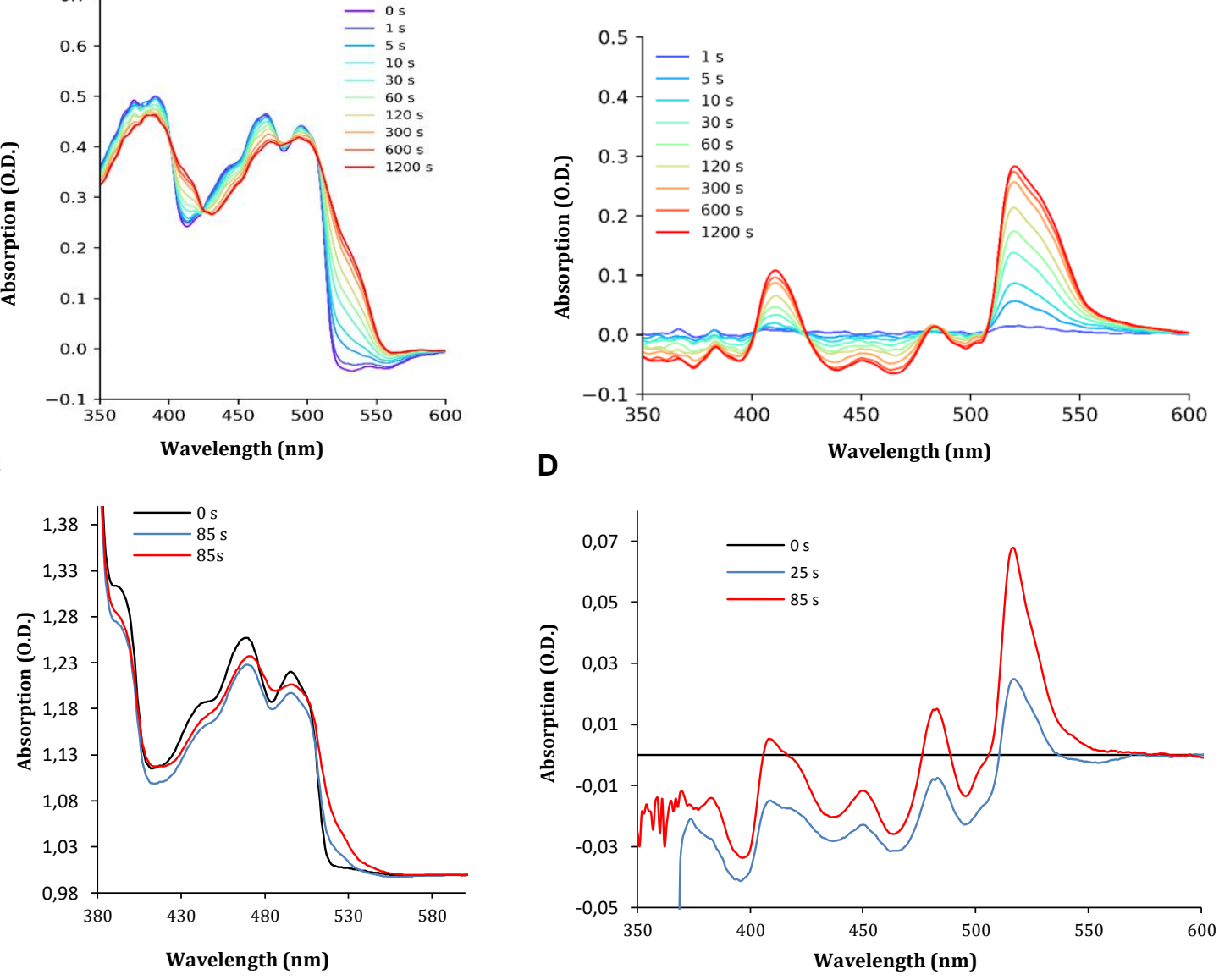

2 Fig. S12: Absorption spectra of the red-shifted intermediate in CvFAP crystals and

3 solution.

4 (A) Series of UV-Vis absorption spectra recorded at $100 \mathrm{~K}$ on a FAP crystal cryoprotected with

$520 \%$ glycerol with increasing irradiation times from a $470 \mathrm{~nm}$ LED. (B) Series of corresponding

6 difference spectra $\left[\operatorname{spectrum}(t)-\operatorname{spectrum}\left(t_{0}\right)\right]$. (C) Series of UV-Vis absorption spectra

7 recorded at $100 \mathrm{~K}$ on a solution of FAP with increasing irradiation times from a $470 \mathrm{~nm}$ LED.

8 (D) Series of corresponding difference spectra $\left[\operatorname{spectrum}(t)-\operatorname{spectrum}\left(t_{0}\right)\right]$. 


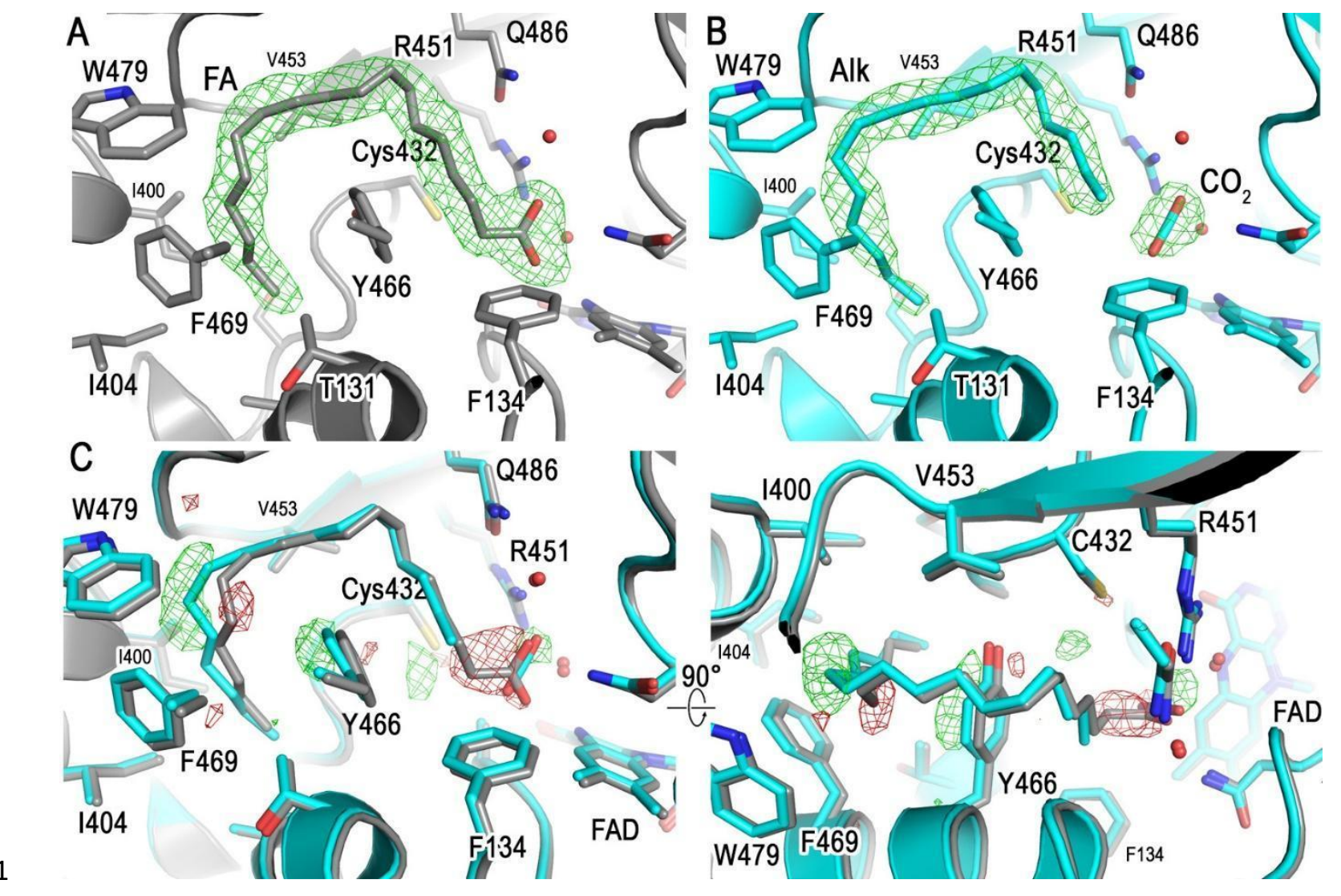

2 Fig. S13: Characterization of the red-shifted intermediate in CvFAP crystals.

3 Structures were obtained from data collected from crystals kept at cryogenic temperature using

4 synchrotron radiation ('100 K light' and '100 K dark'). (A) Omit map around the active site

5 substrate in the dark and $(\mathbf{B})$ after blue light illumination. $(\mathbf{C})$ Experimental difference $\left(F_{\text {light }}-\right.$

$\left.6 \quad F_{\text {dark }}\right)$ map at $100 \mathrm{~K}$ contoured at $+/-4 \sigma$ and centered on the active site substrate, superposed on

7 the refined structures of the dark state (gray) and the red-shifted form (cyan). The cleavage of

8 the $\mathrm{C} 1-\mathrm{C} 2$ bond is clearly visible, together with the recoil of the alkane product toward the

9 exterior along the substrate tunnel, and a rotation of the side chain of Y466 that accompanies

10 this recoil. 


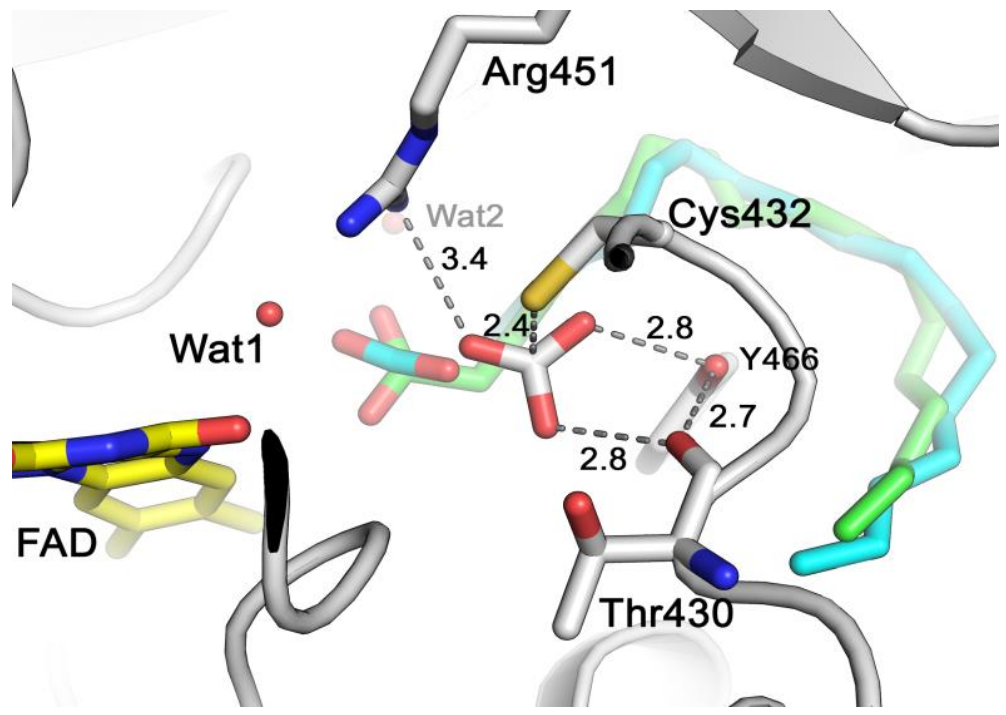

2 Fig. S14: Environment of the bicarbonate modelled in the electron density next to C432 in

3 the $150 \mathrm{~K}$ pH 8.5 structure (' $150 \mathrm{~K}$ light').

4 The environment of the bicarbonate, with putative H-bonds and contacts, is shown. The electron

5 density omit map is shown in Fig. 3D.

6

7

8

9

10

11

12

13 


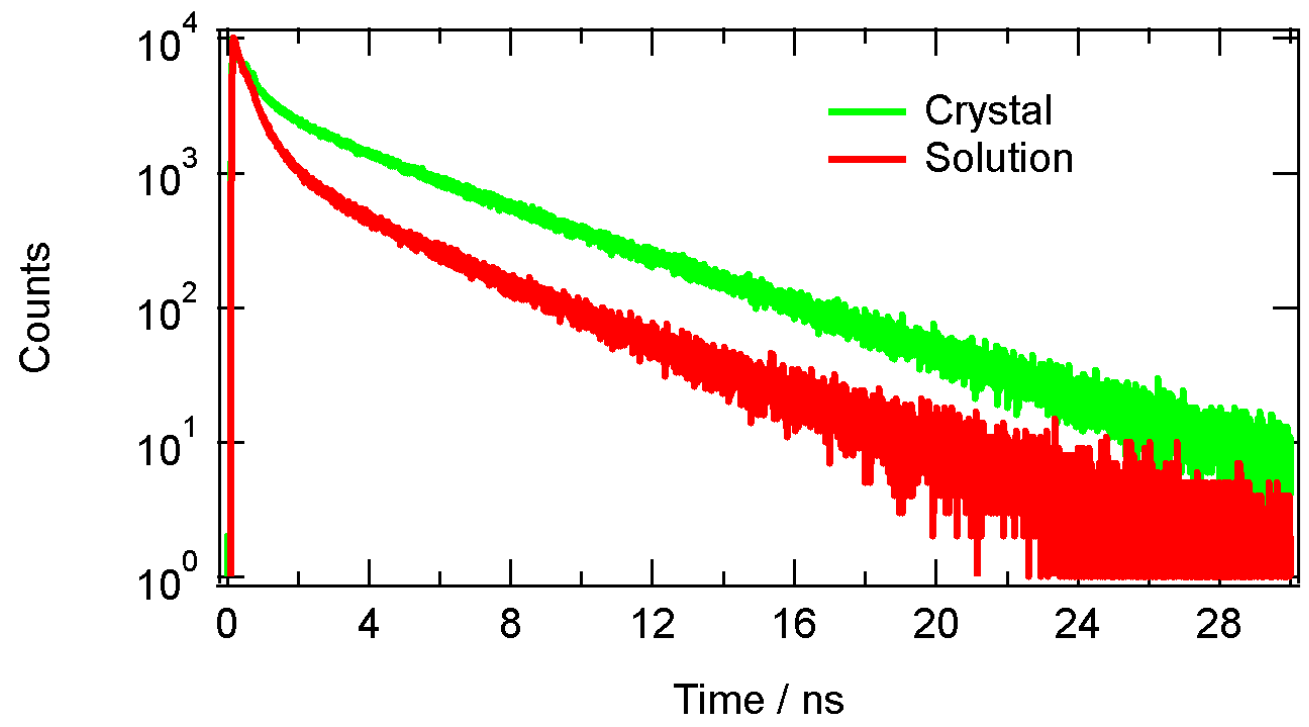

2 Fig. S15: Fluorescence decays measured using single photon counting technique.

3 Decays were measured at $560 \mathrm{~nm}$ after a $400 \mathrm{~nm}$ excitation of FAP in solution (50 mM Tris $\mathrm{pH}$

$48.5,100 \mathrm{mM} \mathrm{NaCl}$ ) and in microcrystals (in 19\% (w/v) PEG 4000, 0.1 M Na citrate pH 5.5, 0.01

5 M spermidine).

6

7

8

9

10

11

12

13

14

15 

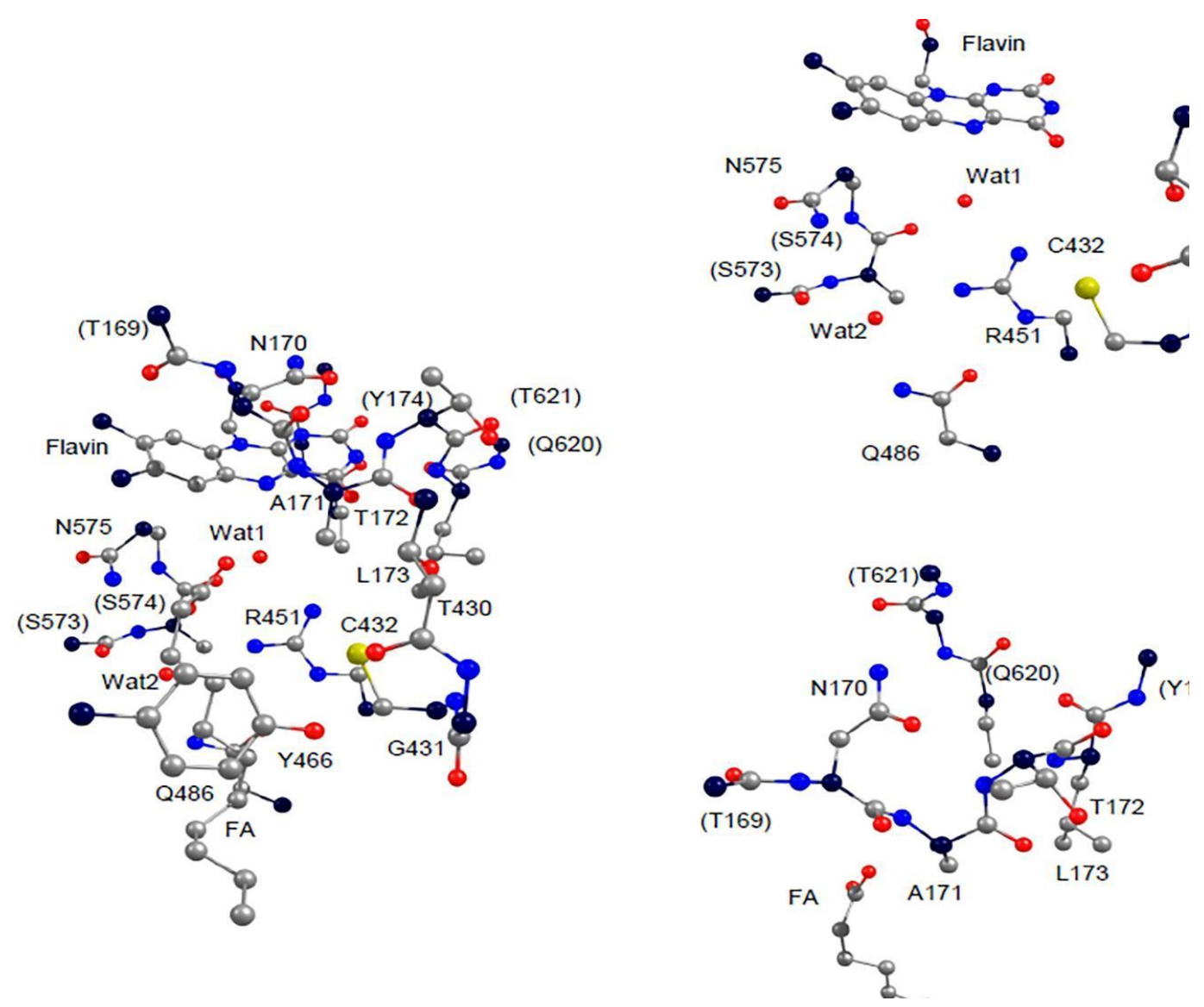

$2 \quad$ Fig. S16: Model of the CvFAP active site.

3 Model of the active site (272 atoms, net charge 0) employed in pathway calculations to obtain

4 the geometries listed in table S5. The hydrogen atoms are not shown. Atoms fixed during

5 geometry optimization are indicated in black. Flavin, fatty acid (FA), water molecules (Wat) and

6 residues are labeled. The residues whose side chain was not included in the model are indicated

7 in brackets. The model consists of the following fragments: flavin, fatty acid (deprotonated),

8 Wat1, Wat2, polypeptide fragments (T169)-N170-A171-T172-L173-(Y174), T430-G431-C432,

9 (S573)-(S574)-N575, (Q620)-(T621), and side chains R451 (protonated), Y466, Q486. On the

10 right-hand side, we show the same model split in two parts for better clarity. 


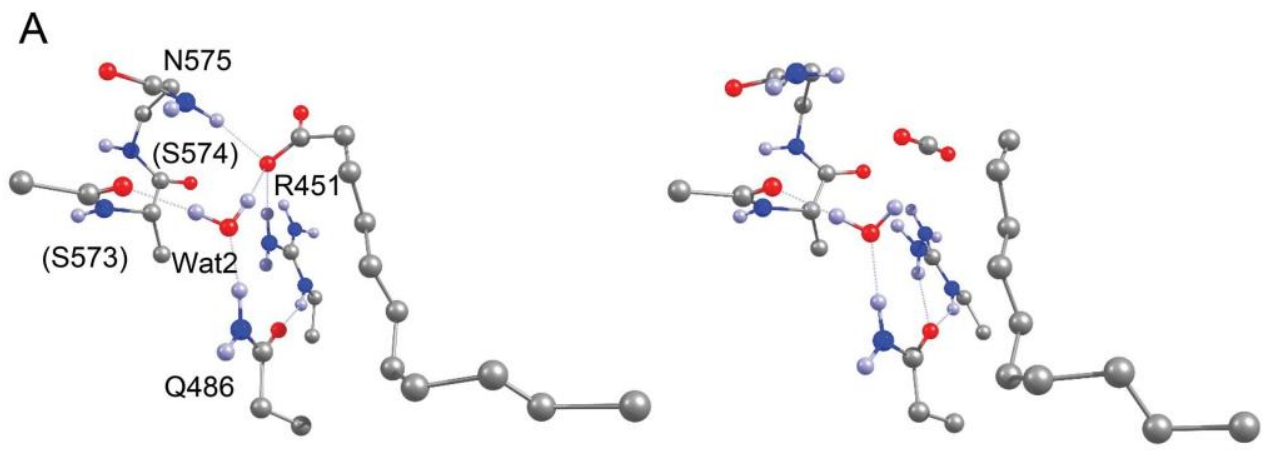

B $\quad \mathrm{CO}_{2}$ dissociation

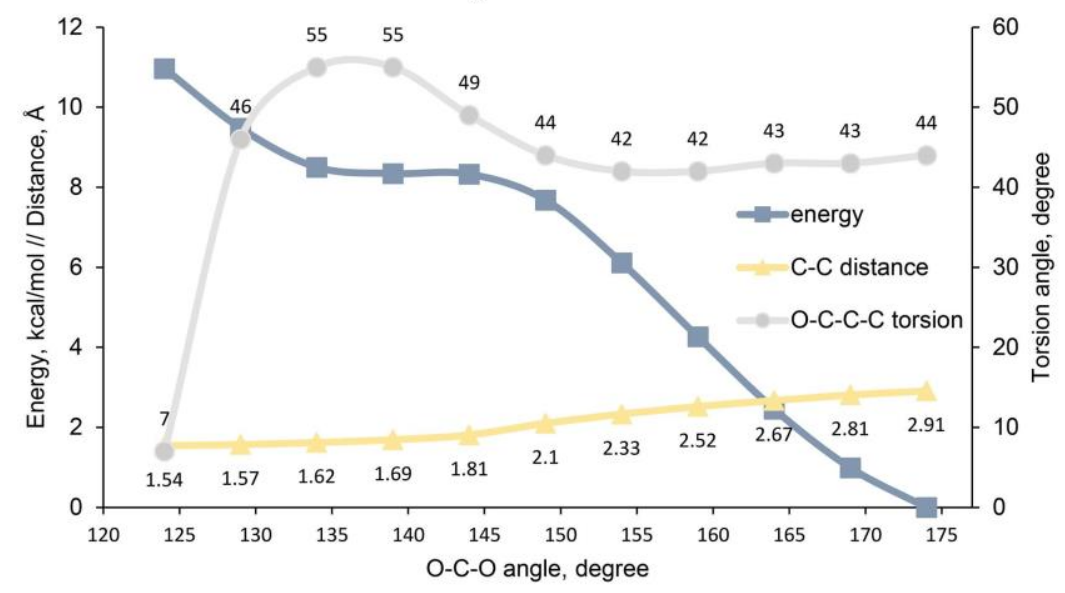

2 Fig. S17: Fatty acid decarboxylation in the charge-transfer (radical) state.

3 (A) The initial and final geometries of the relaxed energy scan in the radical state. A truncated

4 model (94 atoms, net charge 1) was employed in the calculations. The hydrogen atoms bound to

5 the carbon atoms are omitted for clarity. (B) The relaxed energy scan results. The initial

6 geometry was first optimized in the FA anionic state (R-B3LYP/cc-pvdz); after constraining the

7 OCO angle, geometry optimization was continued in the radical state (U-B3LYP/cc-pvdz). All

8 observed changes are triggered by increasing the OCO angle. Relative energy (blue), distance

9 corresponding to the dissociating $\mathrm{C}-\mathrm{C}$ bond (yellow) and $\mathrm{CO}_{2}$ rotation angle (grey) are shown

10 for all partially optimized geometry with the OCO angle fixed at the indicated value. 


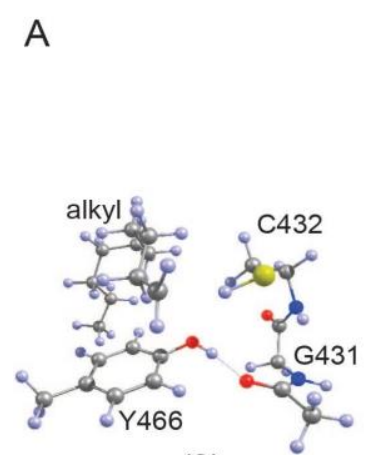

(3)

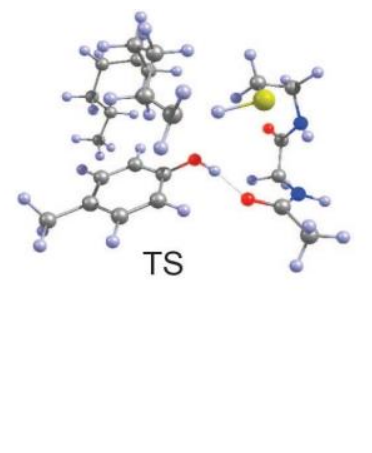

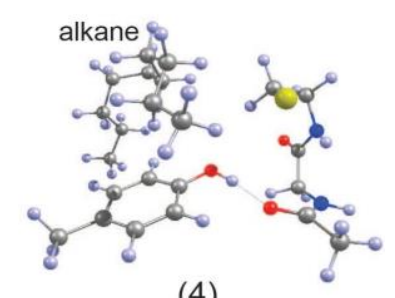

(4)

B

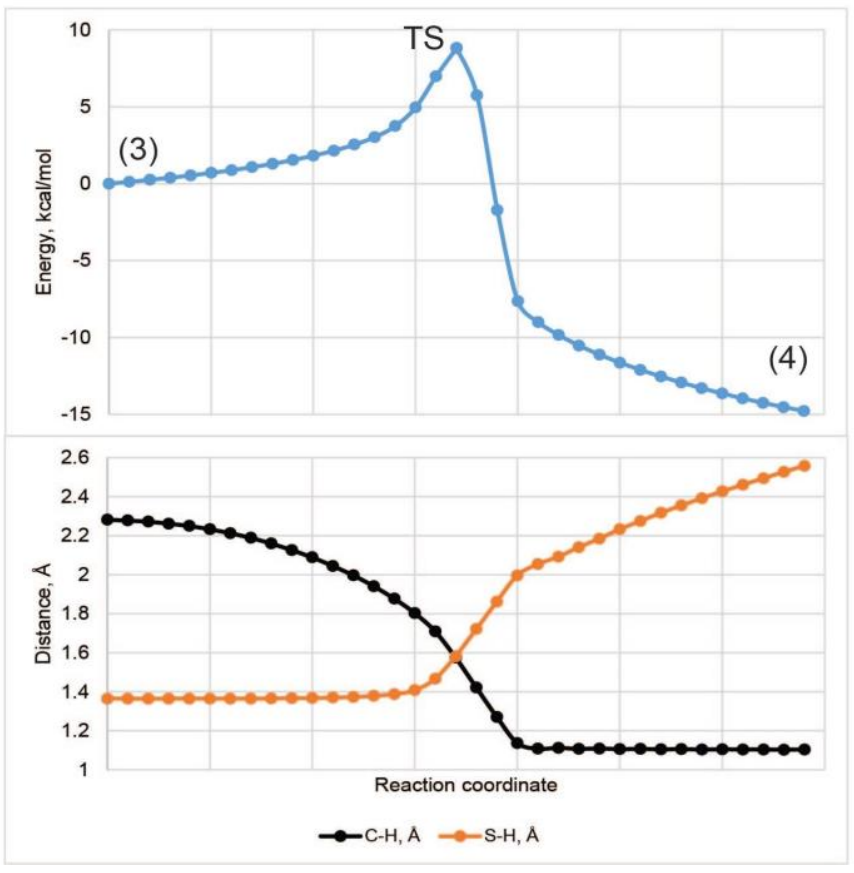

\section{Fig. S18: Energy and transition state of HAT reaction.}

3 (A) The initial (3), final (4) and transition state (TS) geometries describing the HAT reaction.

4 Calculations performed for a truncated model shown in the panel (70 atoms, net charge 0 ) in the

5 radical state, using U-B3LYP/cc-pvdz. The imaginary frequency $\left(1470 \mathrm{i} \mathrm{cm}{ }^{-1}\right)$ mode corresponds

6 to a coupled C-H and H-S stretch. (B) The energy and geometry changes obtained from the

7 intrinsic reaction coordinate (irc) calculations starting from the saddle point geometry (TS)

8 toward (3) and (4). 
A
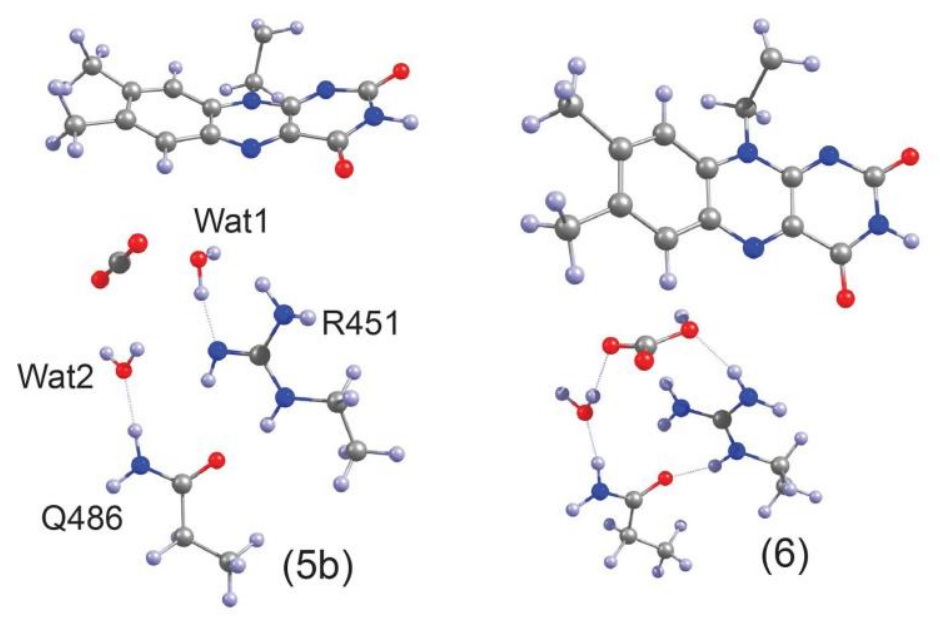

B

Bicarbonate formation involving Wat1
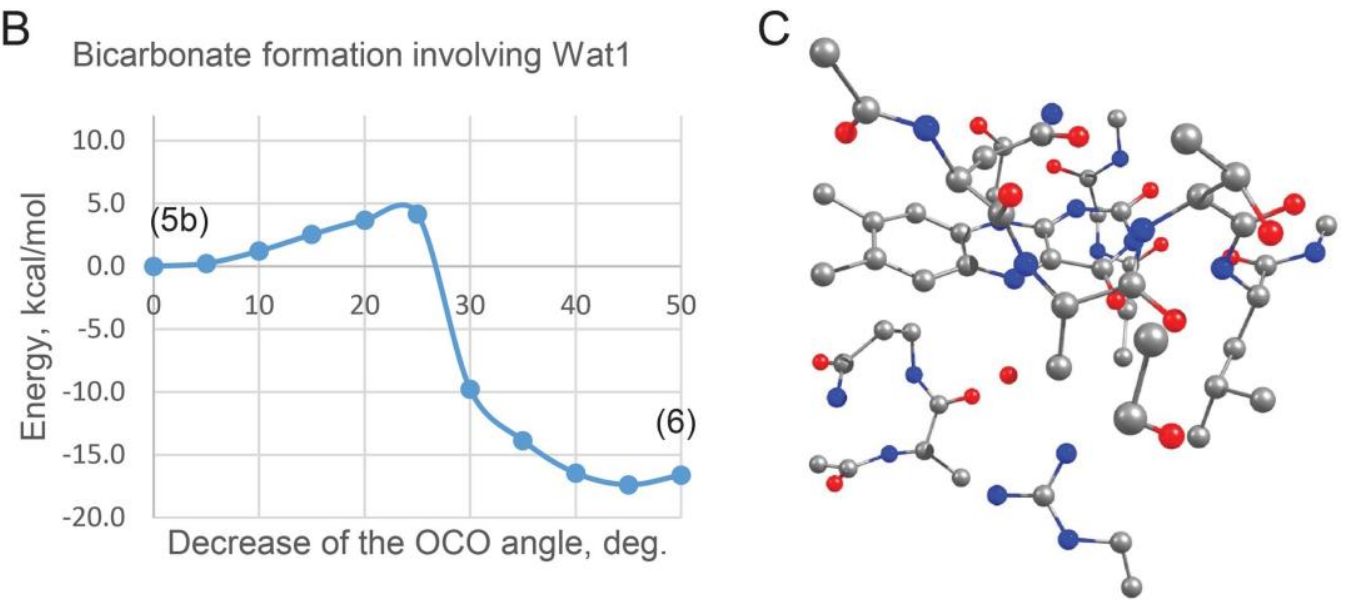

\section{Fig. S19: Bicarbonate formation from $\mathrm{CO}_{2}$ and Wat1.}

3 (A) Bicarbonate formation from Wat1 and carbon dioxide catalyzed by R451 in FAP. Fragments

4 of the model are shown to detail structural changes due to carbon dioxide-bicarbonate

5 conversion. (B) Energies of bicarbonate formation computed with R-B3LYP/cc-pvdz. The

6 relaxed-scan energy calculations were performed along the OCO bending coordinate of carbon

7 dioxide. (C) Truncated active site model employed in the relaxed scan computations addressing

8 bicarbonate formation from Wat1. The hydrogen atoms are omitted for clarity. 

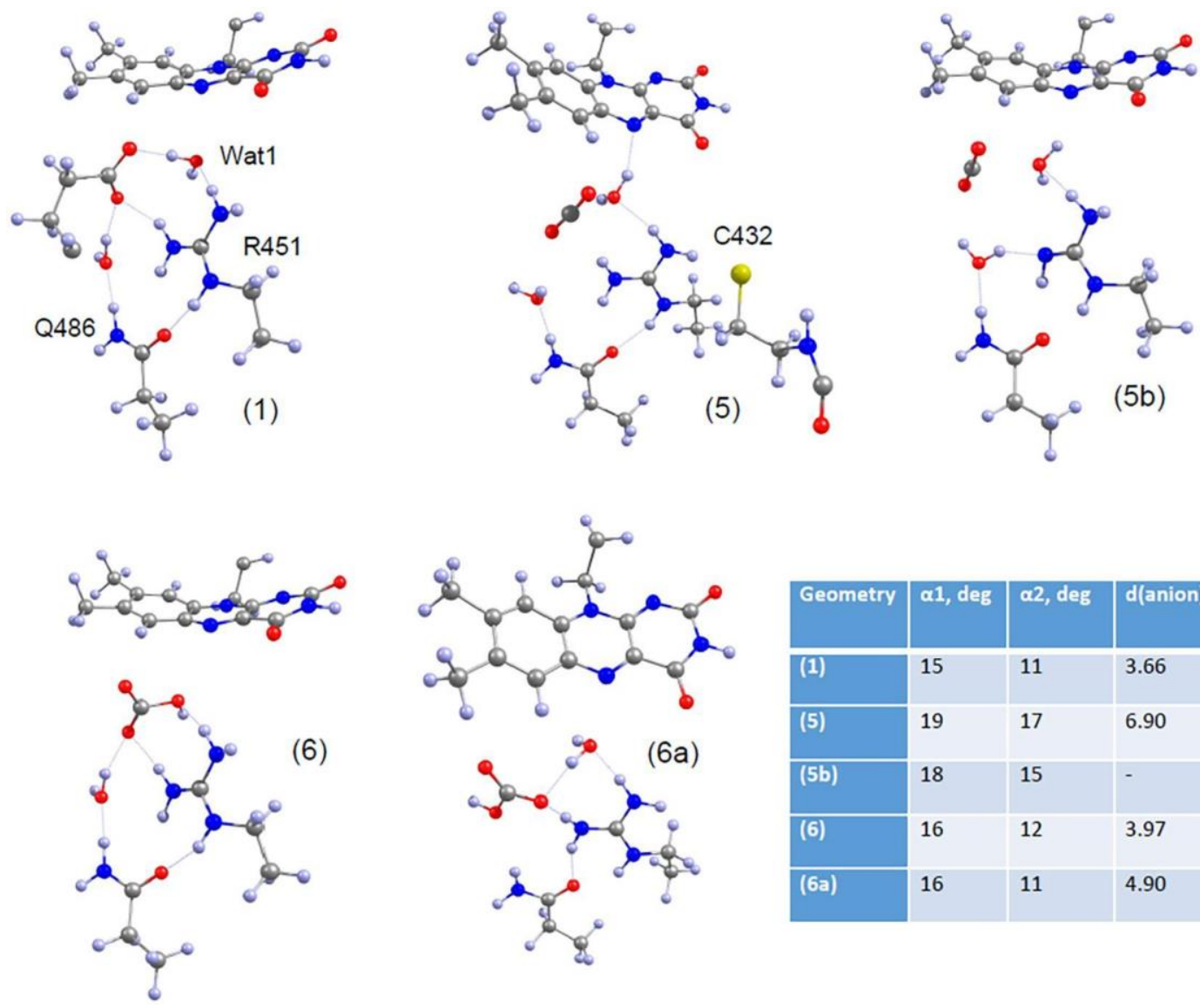

\begin{tabular}{|l|l|l|l|l|}
\hline Geometry & $\alpha 1$, deg & $\alpha 2$, deg & d(anion), A & d(Wat1), A A \\
\hline (1) & 15 & 11 & 3.66 & 2.77 \\
\hline (5) & 19 & 17 & 6.90 & 2.92 \\
\hline (5b) & 18 & 15 & - & 2.97 \\
\hline (6) & 16 & 12 & 3.97 & $3.01(\mathrm{OH})$ \\
\hline (6a) & 16 & 11 & 4.90 & 5.18 \\
\hline
\end{tabular}

2 Fig. S20: Suggested red-shifted FAD

3 Flavin bending is defined by angles $\alpha 1(\mathrm{C} 9 \mathrm{~N} 10 \mathrm{~N} 5 \mathrm{C} 4)$ and $\alpha 2(\mathrm{C} 6 \mathrm{~N} 5 \mathrm{~N} 10 \mathrm{~N} 1)$; $\mathrm{d}($ anion) is the

4 shortest distance from flavin $\mathrm{N} 5$ to the anionic species $\left(\mathrm{R}-\mathrm{CO}_{2}{ }^{-}, \mathrm{HCO}_{3}{ }^{-}\right.$and $\left.\mathrm{C} 432^{-}\right)$; $\mathrm{d}(\mathrm{Wat} 1)$ is

5 the distance from favin N5 to Wat1 oxygen. 


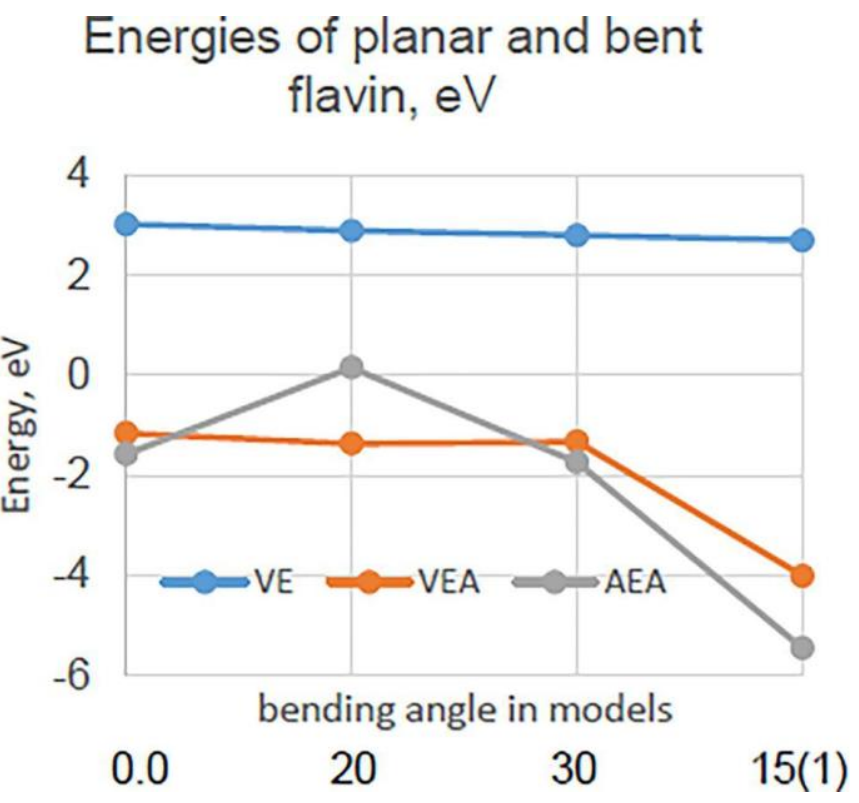

$2 \quad$ Fig. S21: Flavin butterfly bending energies.

3 The energies compared in this figure are listed in table S9. The negative vertical electron affinity

4 (VEA) and adiabatic electron affinity (AEA) energies indicate that flavin semiquinone has a

5 lower energy (electronically more stable) than the oxidised state. Flavin bending decreases the

6 vertical excitation (VE) energy. The decrease of the AEA value indicates stabilization of flavin

7 semiquinone against re-oxidation. At a 15-degree bent geometry and in the active-site model (1)

8 (bending angle 15(1) in the figure), the VEA value decreases to a larger extent than VE energy

9 (in comparison to the planar geometry), indicating stabilization of CT states (flavin

10 semiquinone) and hence, favoring fET. Notably, the effect of bending is enhanced by

11 intermolecular interactions in the FAP active-site model (1). Hence, flavin bending (along with

12 intermolecular interactions) red-shifts the absorbance of the oxidized flavin and facilitates fET. 

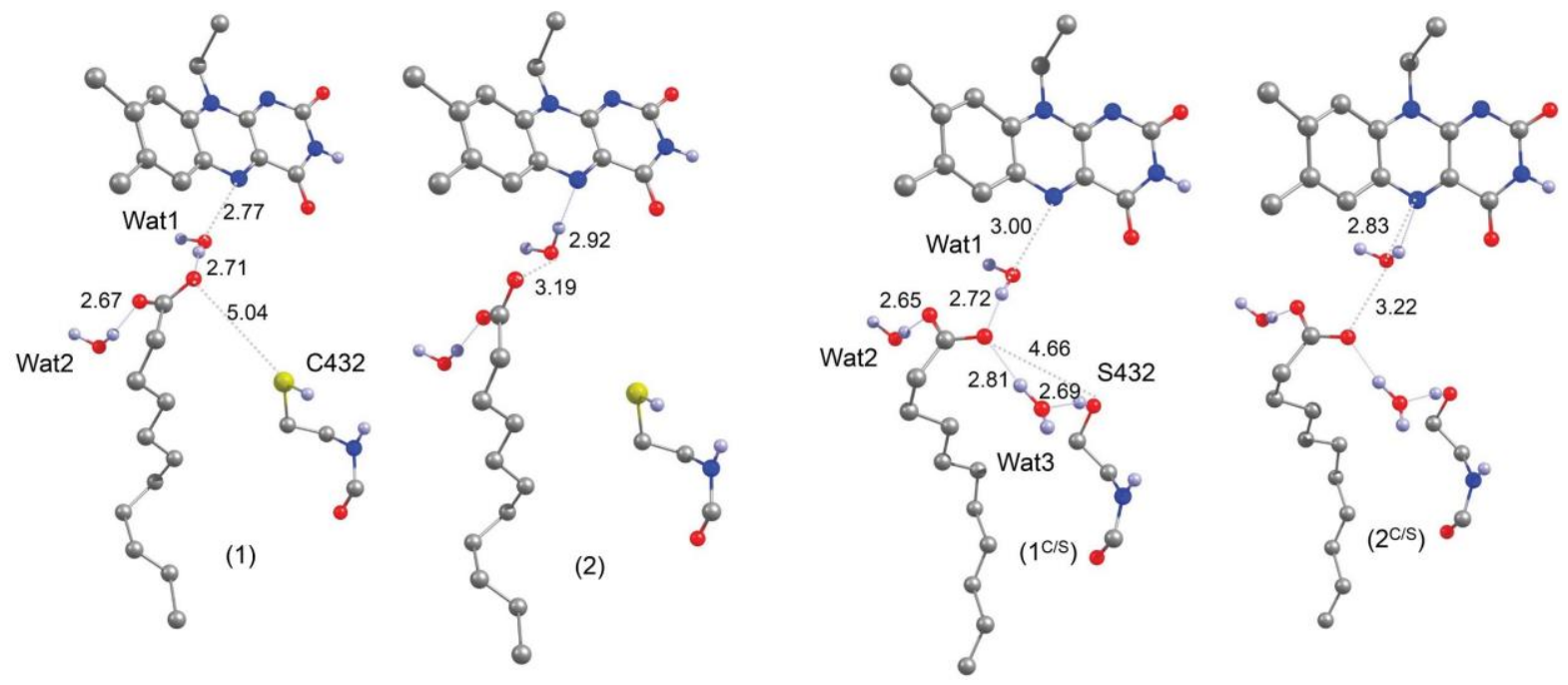

2 Fig. S22: Interaction of the fatty acid carboxylate with water molecules in wild type and

\section{C432S models.}

4 Distances $(\AA)$ characterize interactions of the fatty acid substrate with the active-site water 5 molecules. 


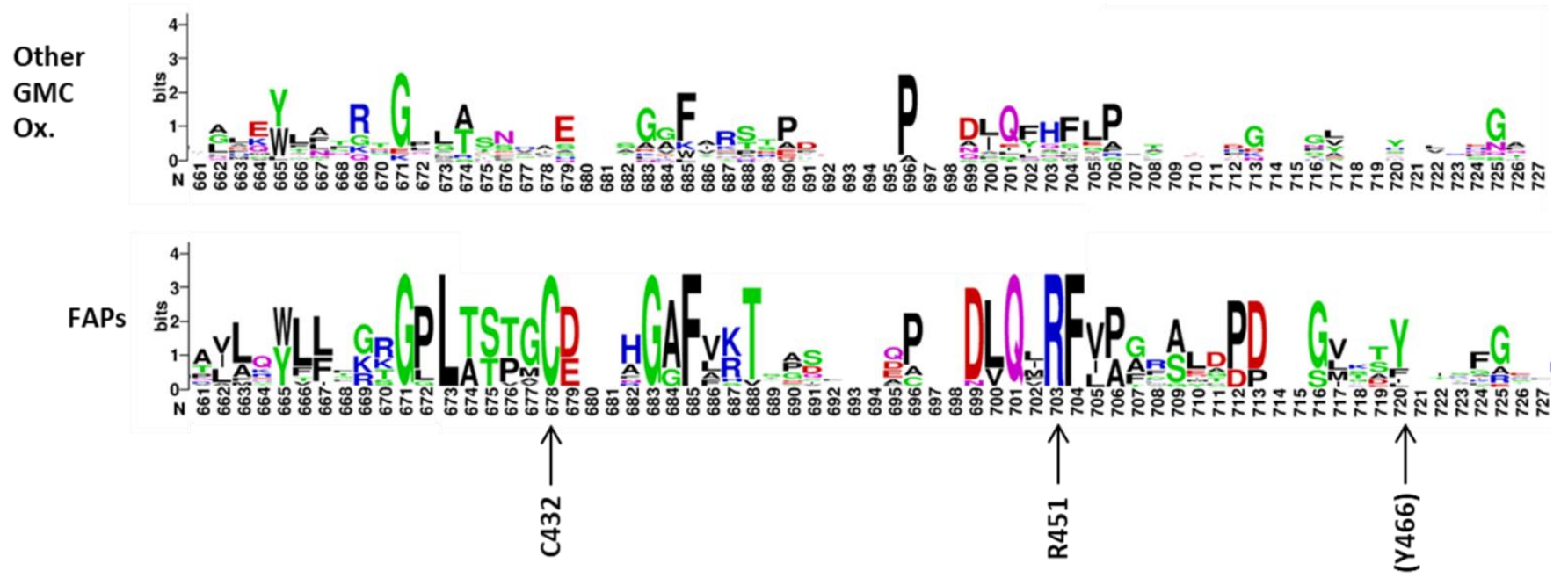

2 Fig. S23: Conservation of residues specific to FAPs within the active site of the GMC

3 oxidoreductases.

4 Protein sequences of 14 FAPs and 16 other GMC oxidoreductases (table S4) were aligned and a

5 sequence logo generated. Only the part of the sequence logo featuring the possible proton donors

6 to the alkyl radical is shown (C432, R451, Y466 in $C v F A P)$. Letters represent amino acids at

7 successive positions in the multiple alignment. The overall height of the stack indicates the

8 sequence conservation at that position, while the height of symbols within the stack indicates the

9 relative frequency of each amino acid at that position. The sequence logo highlights that C432

10 and R451 are $100 \%$ and specifically conserved in FAPs . 
A

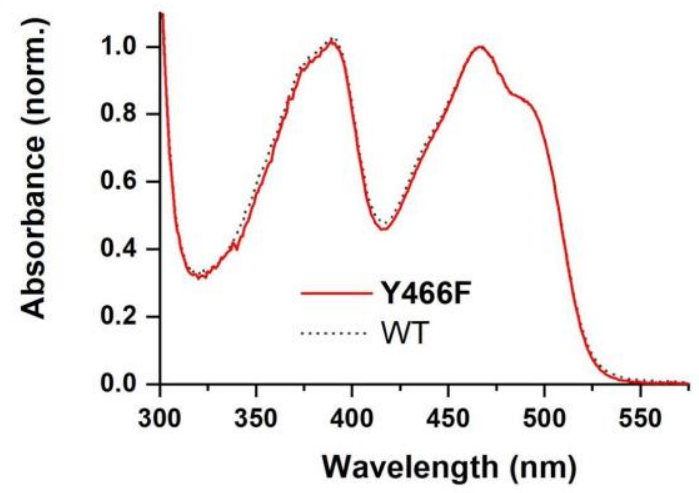

C

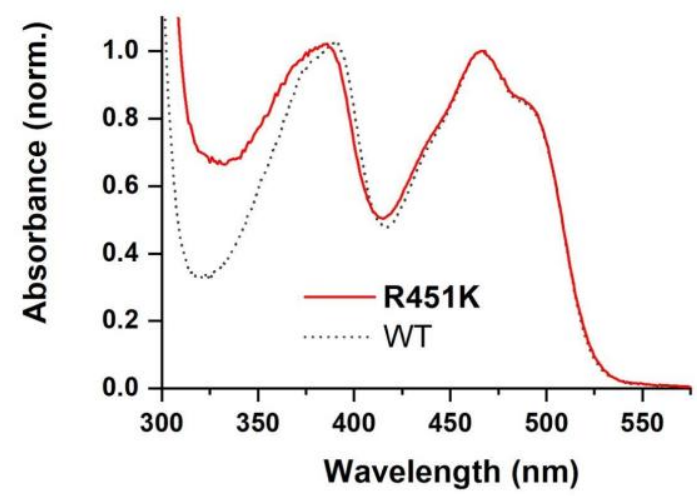

B

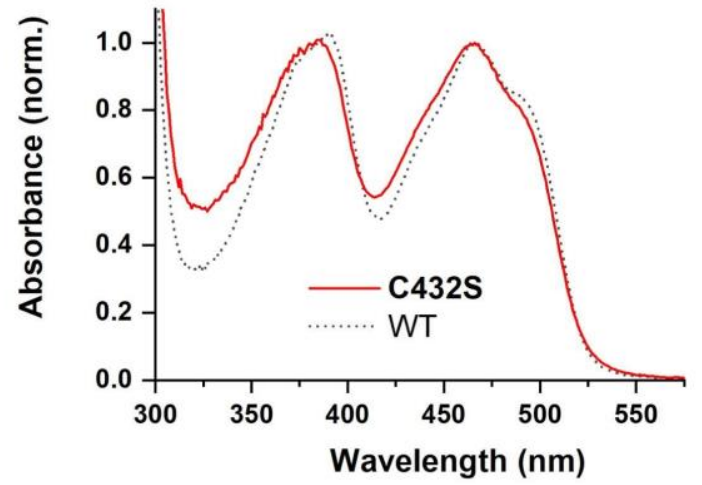

D

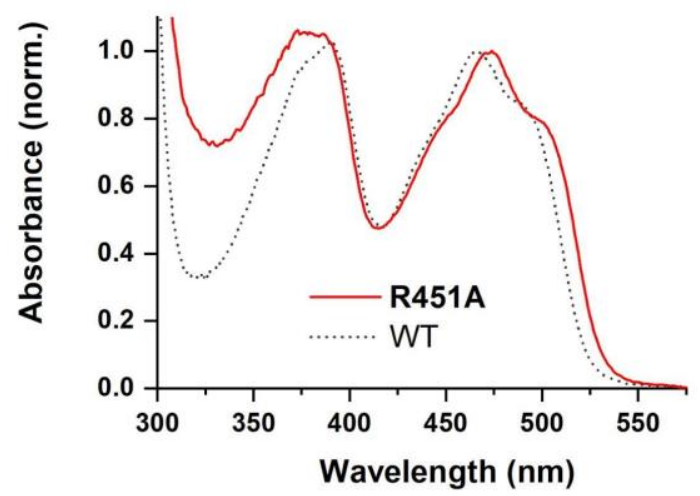

1

2 Fig S24: UV-Vis spectra of WT and mutant $C \nu$ FAP.

3 UV-Vis spectra of Y466F (A), C432S (B), R451K (C) and R451A (D) mutants compared to

4 WT. The absorption maximum in the C432S mutant is $2 \mathrm{~nm}$ blue-shifted and that of R451A is 9

5 nm red-shifted compared to WT.

6

7

8

9

10

11 
A
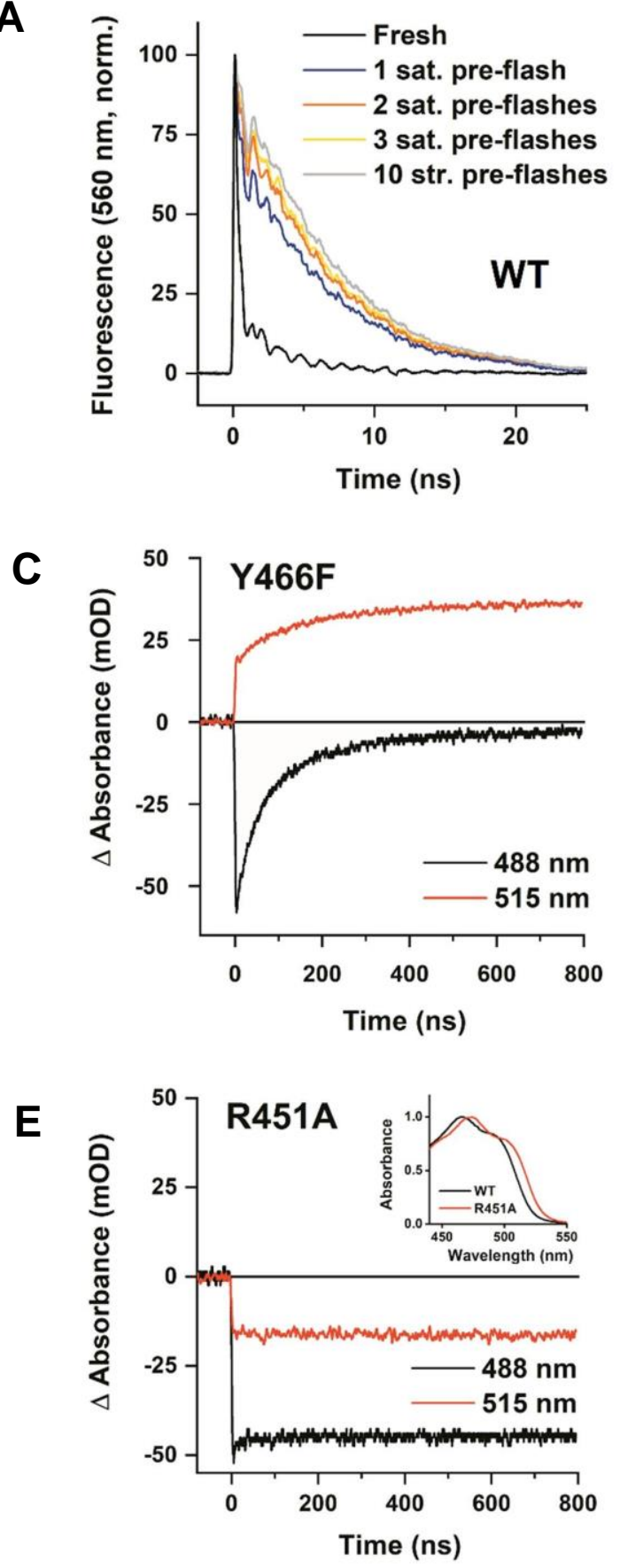
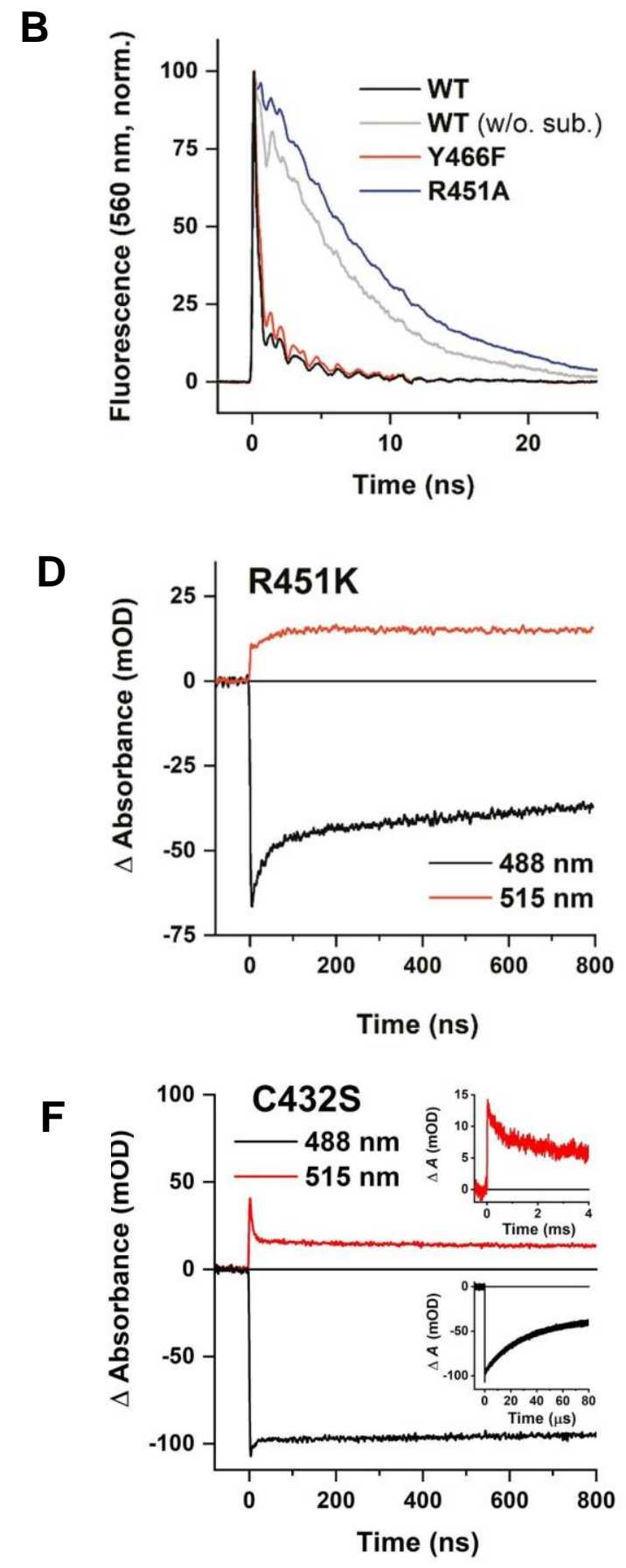

2 Fig. S25: Time-resolved fluorescence and transient absorption signals of WT and mutant

\section{$3 \quad$ CvFAP.}

4 (A) Effect of the native substrate(s) consumption by saturating $(\sim 10 \mathrm{~mJ})$ and strong $(\sim 5 \mathrm{~mJ})$ pre-

5 flashes at $470 \mathrm{~nm}$ on time-resolved ${ }^{1} \mathrm{FAD}^{*}$ fluorescence decay in WT $C v \mathrm{FAP}$ at $560 \mathrm{~nm}$ 
1 (normalized). (B) Comparison of normalized time-resolved ${ }^{1} \mathrm{FAD} *$ fluorescence in $\sim 45 \mu \mathrm{M}$ WT

2 (before and after consumption of the native substrates) and in $\sim 40 \mu \mathrm{M} \mathrm{Y} 466 \mathrm{~F}$ and $\mathrm{R} 451 \mathrm{~A} C \nu \mathrm{FAP}$

3 (in the presence of native substrates); for C432S and R451K mutants, see Fig. 7B. (C) Kinetics

4 of $\mathrm{FAD}^{\bullet-}$ reoxidation in $\sim 40 \mu \mathrm{M}$ Y466F mutant monitored at $488 \mathrm{~nm}$ and $515 \mathrm{~nm}$. (D) Kinetics

5 of $\mathrm{FAD}^{\bullet-}$ reoxidation in $\sim 40 \mu \mathrm{M}$ R451K mutant. (E) Transient absorption changes recorded on

6 the sub-microsecond timescale for $\sim 40 \mu \mathrm{M}$ R451A $C v \mathrm{FAP}$. Inset: comparison of FAD

7 absorption spectra in WT and R451A $C v$ FAP. (F) Transient absorption changes recorded on the

8 sub-microsecond timescale for $\sim 70 \mu \mathrm{M}$ C432S $C \nu$ FAP. Lower inset: decay of the signal at 488

$9 \mathrm{~nm}$ on the microsecond timescale. Upper iInset: decay of the signal at $515 \mathrm{~nm}$ on the millisecond 10 timescale. 
1

2

3

4

5

6

7

8

9

10

11

12

13

14

15

16

17

13

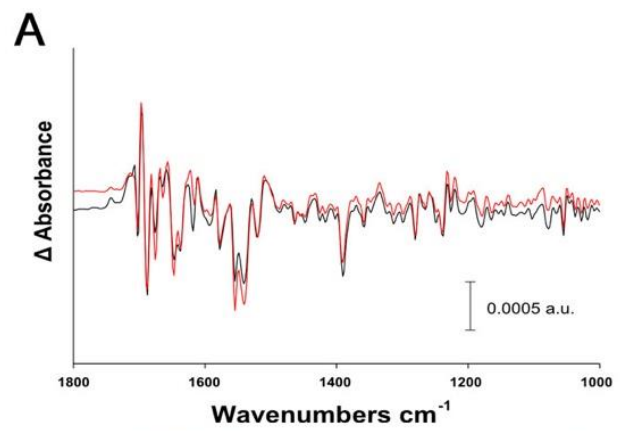

B
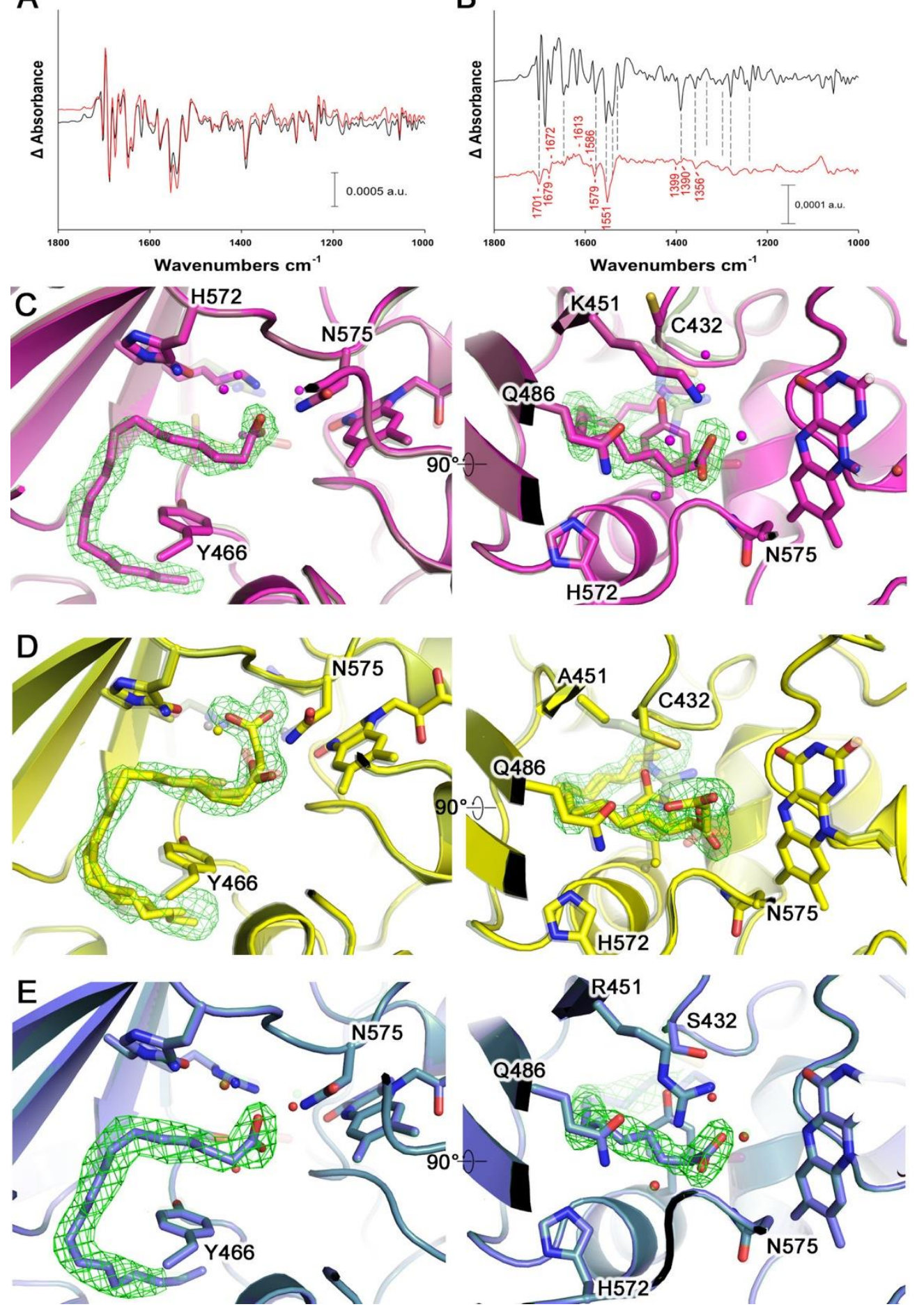
1 Fig. S26: Comparison of the light-induced FTIR difference spectra of $C v$ FAP and $C v$ FAP

2 mutants recorded at $100 \mathrm{~K}$, and omit map electron density maps of $\mathrm{R} 451 \mathrm{~K}, \mathrm{R} 451 \mathrm{~A}$ and

\section{$3 \quad$ C432S mutants.}

4 (A) FTIR spectra of $C \nu$ FAP WT (black) and Y466F mutant (red). (B) FTIR spectra of $C \nu$ FAP

5 and $\mathrm{R} 451 \mathrm{~K}$ mutant (red). The size of the $C \nu \mathrm{FAP}$ spectrum was divided by a factor of $\sim 6$ for the

6 comparison. (C) Structure of the R451K mutant (purple) superimposed with the structure of WT

7 (dark and semi-transparent). Note the rotation of the carboxylate compared to the WT. (D)

8 Structure of the R451A mutant (yellow) superimposed with the structure of WT (dark and semi-

9 transparent). Note the deeper penetration of the fatty acid substrate in the space left by the

10 missing side chain or R451 and the impossibility to stabilise Wat1. (E) Structure of the C432S

11 mutant (blue) superimposed with the structure of WT (dark and semi-transparent). The $F_{\mathrm{o}}-F_{\mathrm{c}}$

12 omit map (contoured at $3 \sigma$ level) corresponding to the fatty acid substrate is shown in green for 13 panels C-E. 


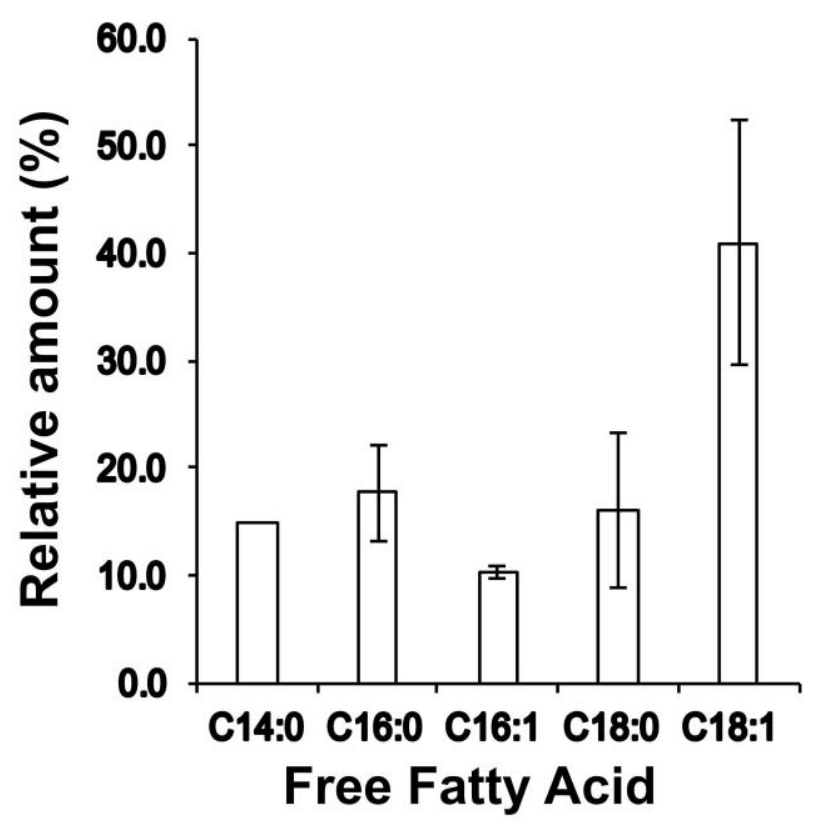

1

2 Fig. S27: Analysis of the fatty acids associated with purified WT $C v F A P$.

3 Fatty acids co-purified with WT $C \nu$ FAP were extracted and the composition was analyzed by

$4 \quad$ LC-MS. Mean \pm SD $(n=3)$.

5

6

7

8

9

10

11

12

13 
A

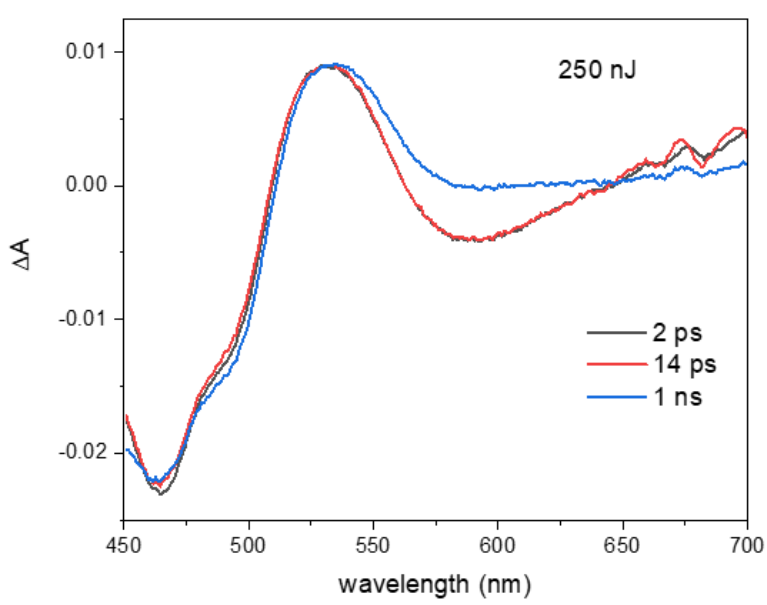

C

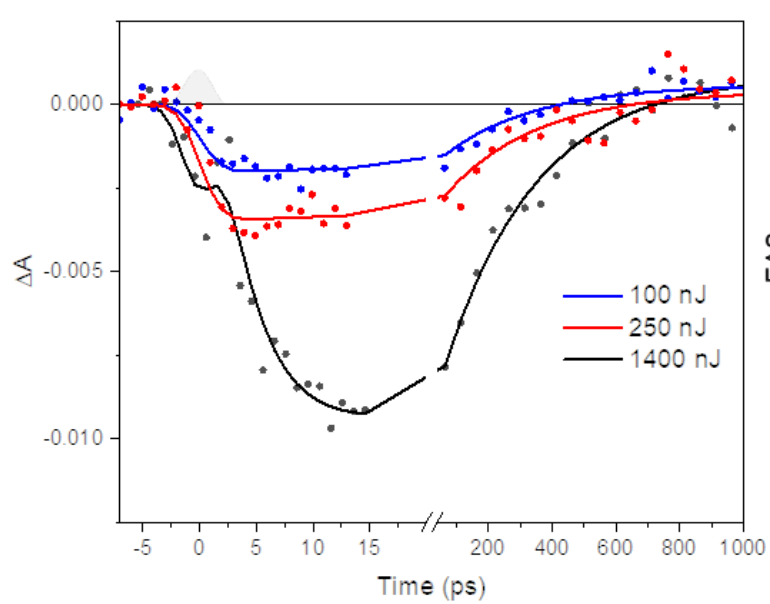

B

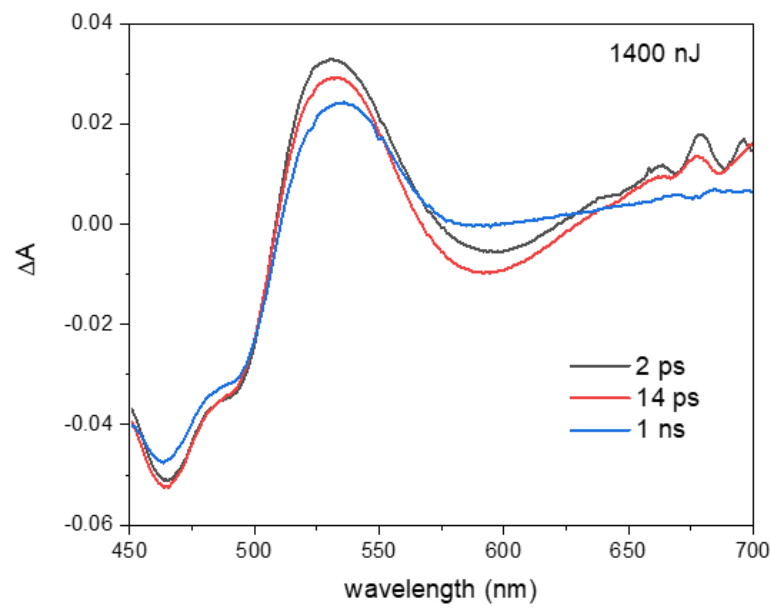

D

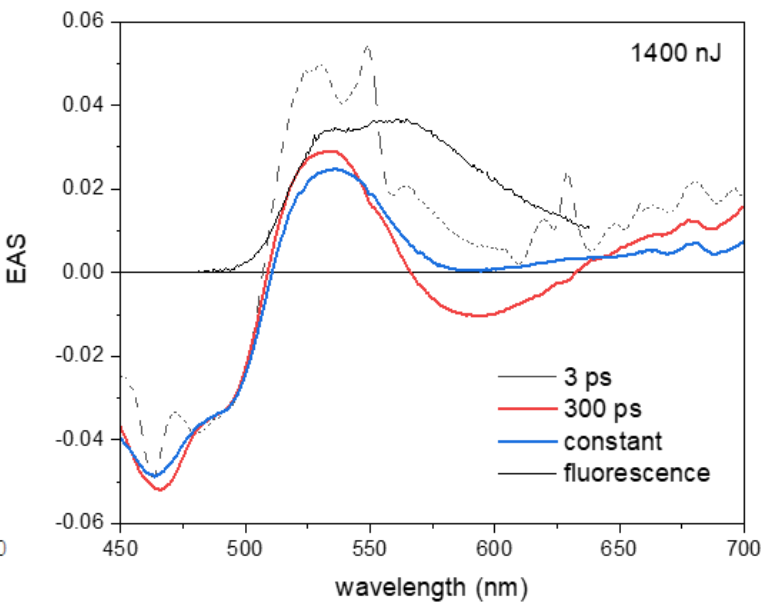

2 Fig. S28: Excitation power dependence of transient absorption with picosecond excitation

\section{3 pulses.}

4 To investigate possible multiphoton effects under the conditions of the SFX experiments,

5 transient absorption experiments were performed with 2.7-ps pulses (temporal profile in grey in

6 panel C) with energies in the range 100-1400 nJ; the highest energy corresponds to average

7 power density of $\sim 10 \mathrm{GW} / \mathrm{cm}^{2}$ and absorption of $\sim 2.3$ photons/flavin in the excitation volume.

8 The overall shape of the transient spectra is very similar for 100 and $250 \mathrm{~nJ}$, but only at higher 
1 powers in the range $520-620 \mathrm{~nm}$ decay of a more positive signal between 2 ps and 14 ps is

2 observed (A-B). Consistently, whereas the global analysis of the data in terms of a

3 multiexponential model required only a 300-ps and a constant phase at low energy (as with the

4 low-energy 100-fs excitation pulses, fig. S6D), a $\sim 3$ ps additional phase was required for high

5 energy (panel C-D; the strong wiggles around 470, 550 and $630 \mathrm{~nm}$ in the 3-ps EAS correspond

6 to cross-phase modulation artifacts that are difficult to eliminate from the fit given that the pulse

7 width is similar to the 3-ps rise time). The spectral evolution associated with this phase

8 (difference between 3-ps and 300-ps EAS) has a similar shape as the FAP fluorescence spectrum

9 (black line) suggesting it represents a rise in the stimulated emission. Altogether it appears that

10 under multiphoton excitation there is a $\sim 3$ ps rise component of the $\mathrm{S} 1 \rightarrow \mathrm{S} 0$ emission, whereas

11 it occurs instantaneously at sub-stoichiometric excitation. We assign this to excitation to higher

12 electronic levels by absorption of multiple photons, and subsequent internal conversion to and

13 cooling in the S1 level. These processes occur on a much shorter timescale than the $\sim 300$ ps ET

14 time that appears not sensitive to the excitation power. The small differences in shape of the 1-

15 ns spectra (long-lived component) at $1400 \mathrm{~nJ}$ and $250 \mathrm{~nJ}$ can be assigned to a fraction of long-

16 lived $\mathrm{FAD}^{*}$ due to substrate depletion at very high excitation power. No evidence was found for

17 further flavin photoproducts. 

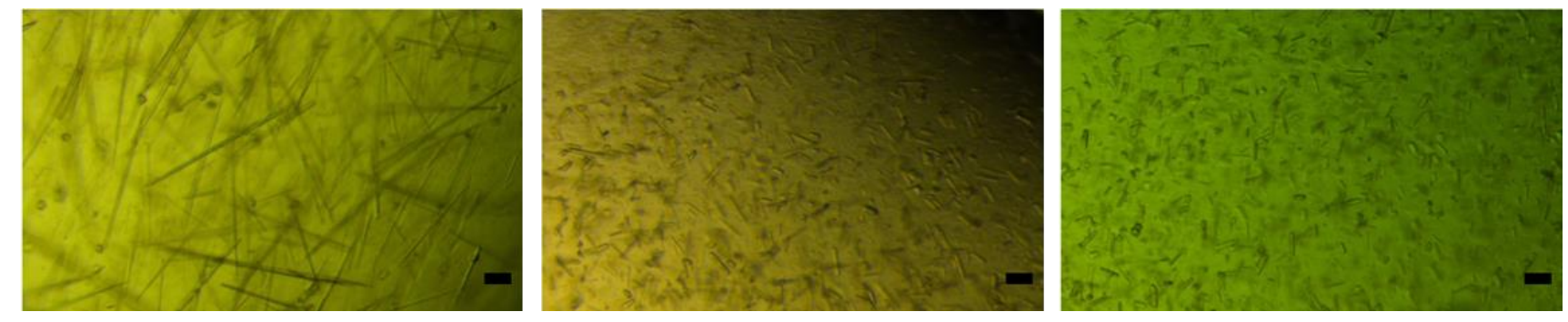

2

3 Fig. S29: Filtering effect on FAP microcrystal size.

4 Before filtering, the crystal size was about $100-200 \times 2-5 \times 2-5 \mu \mathrm{m}^{3}$ (left). After flowing

5 them through a $20 \mu \mathrm{m}$ (middle) and then a $10 \mu \mathrm{m}$ filter the size was $10 \times 2-5 \times 2-5 \mu \mathrm{m}^{3}$

6 (right). The scale bar corresponds to $40 \mu \mathrm{m}$.

7

8

9

10

11

12

13

14

15

16 


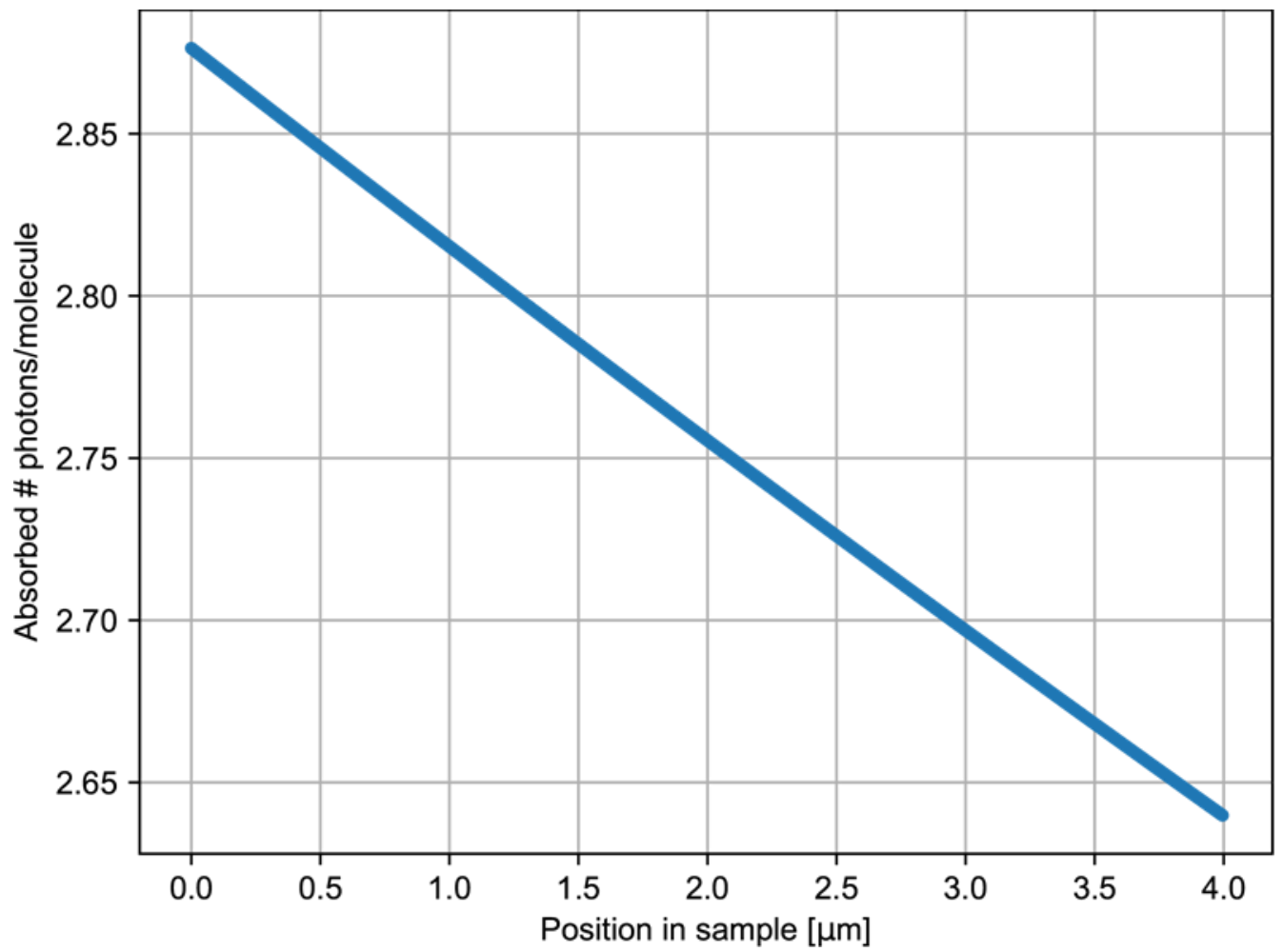

3 Fig. S30: Depth-dependent photon absorption regimes inside FAP crystals

4 Depth-dependent photon absorption $\left(9250 \mathrm{M}^{-1} \mathrm{~cm}^{-1}\right.$ at $\left.400 \mathrm{~nm}\right)$ regime in a 4- $\mu \mathrm{m}$ FAP crystal

5 (FAD concentration $10.3 \mathrm{mM}$ ) with pump-laser illumination (4 ps pulse length, $11 \mu \mathrm{J}$ pulse 6 energy, $155 \mu \mathrm{m}$ (FWHM) spot size). The 1/e penetration depth is $47 \mu \mathrm{m}$. 


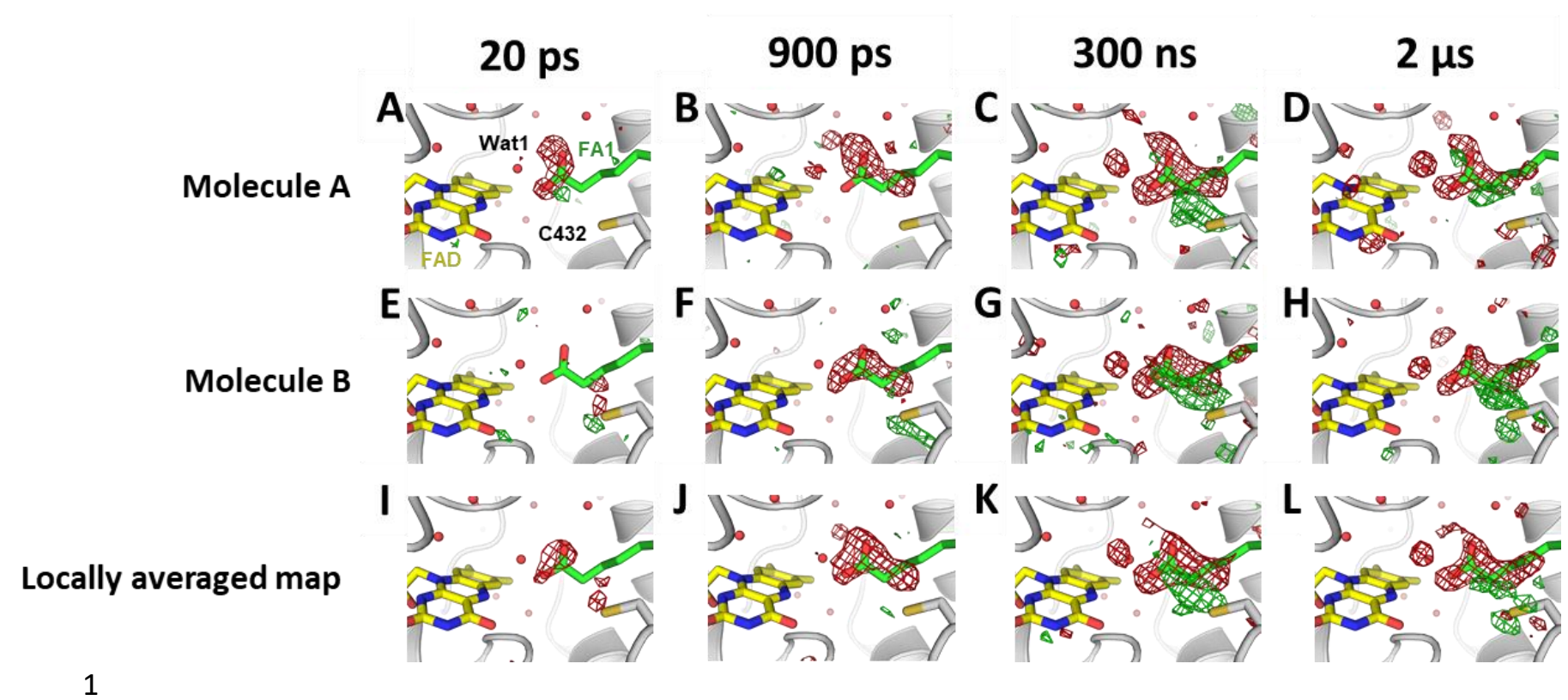

2 Fig. S31: Time-dependent changes in the $C v$ FAP active site by TR-SFX

3 Q-weighted difference Fourier electron density maps calculated between the SFX light and dark

4 data sets $\left(F_{\mathrm{obs}}{ }^{\Delta t}-F_{\mathrm{obs}}{ }^{\text {dark}}\right.$; with $\Delta t=20 \mathrm{ps}(A, E, I), 900 \mathrm{ps}(B, F, J), 300 \mathrm{~ns}(\boldsymbol{C}, \boldsymbol{G}, \boldsymbol{K}), 2 \mu \mathrm{s}(\boldsymbol{D}$,

$5 \boldsymbol{H}, \boldsymbol{L}))$ at $2.2 \AA$ resolution. Maps corresponding to molecules $\mathrm{A}(\boldsymbol{A}-\boldsymbol{D})$ and $\mathrm{B}(\boldsymbol{E}-\boldsymbol{H})$ are shown at

$6+3.5 \sigma$ (green) and $-3.5 \sigma$ (red) and locally NCS-averaged maps $(I-L)$ at $+4 \sigma$ (green) and $-4 \sigma$

7 (red). The SFX dark-state model (FAD in yellow, fatty acid in green, protein moiety in light

8 grey) of molecule $\mathrm{A}$ is overlaid in panels $A-D$ and of molecule $\mathrm{B}$ in panels $E-L$. The maps were

9 calculated from 68,421 dark images and 88,919, 50,214, 44,868 and 18,600 light images for $\Delta t$

$10=20$ ps, 900 ps and $300 \mathrm{~ns}$ and $2 \mu$ s, respectively. Panels $I-L$ are identical to those in Fig. 5. 


\section{Supplementary Tables}

2 Table S1. Crystal data, data-collection and refinement statistics.

\begin{tabular}{|c|c|c|c|c|c|c|c|}
\hline \multicolumn{8}{|l|}{ Data collection } \\
\hline $\begin{array}{l}\text { Data Set Name } \\
\text { PDB code }\end{array}$ & $\begin{array}{l}\text { Dark } \\
\text { 6YRU }\end{array}$ & $\begin{array}{l}\text { Red-shift } \\
\text { 6YRV }\end{array}$ & $\begin{array}{l}\text { Low-Dose } \\
\text { 6YRX }\end{array}$ & $\begin{array}{l}\text { pH8.5 } \\
\text { 6YRZ }\end{array}$ & $\begin{array}{l}\text { R451K } \\
\text { 6YS1 }\end{array}$ & $\begin{array}{l}\text { R451A } \\
6 Y S 2\end{array}$ & \\
\hline Beamline & ID29/ESRF & ID29/ESRF & $\begin{array}{l}\text { ID30- } \\
\text { A3/ESRF }\end{array}$ & $\begin{array}{l}\text { PX1/SOLE } \\
\text { IL }\end{array}$ & $\begin{array}{l}\text { ID29/ESR } \\
\text { F }\end{array}$ & $\begin{array}{l}\text { ID29/ESR } \\
\mathrm{F}\end{array}$ & ; \\
\hline Dark/Light & Dark & Light & Dark & Light & Dark & Dark & \\
\hline $\mathrm{pH}$ & 5.5 & 5.5 & 5.5 & 8.5 & 5.5 & 5.5 & \\
\hline Temperature & $100 \mathrm{~K}$ & $100 \mathrm{~K}$ & RT & $150 \mathrm{~K}$ & $100 \mathrm{~K}$ & $100 \mathrm{~K}$ & \\
\hline Space Group & 1222 & 1222 & 1222 & 1222 & $P 2_{1} 2_{1} 2_{1}$ & 1222 & \\
\hline \multicolumn{8}{|l|}{ Unit-cell parameters ( $(\AA)$} \\
\hline $\mathrm{a}$ & 89.7 & 89.9 & 95.6 & 90.5 & 58.9 & 89.6 & \\
\hline $\mathrm{b}$ & 103.6 & 103.7 & 105.7 & 104.6 & 60.8 & 102.8 & \\
\hline $\mathrm{c}$ & 155.9 & 156.3 & 158.0 & 156.9 & 180.1 & 155.8 & \\
\hline 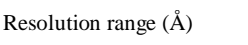 & $86-1.78$ & $86-1.94$ & $88-1.87$ & $87-1.82$ & $90-1.97$ & $86-1.64$ & \\
\hline High resolution range $(\check{A})$ & $1.9-1.78$ & $2-1.94$ & $2-1.87$ & $1.93-1.82$ & $2.1-1.97$ & $1.75-1.64$ & . \\
\hline Observed reflections & 327,307 & 359,526 & 278,791 & 895,789 & 225,269 & 370,900 & \\
\hline No. of unique reflections & 69,240 & 54,600 & 65,032 & 66,267 & 46,463 & 87,644 & \\
\hline Completeness (\%) & $99.3(99.4)$ & $100(100)$ & $98.1(99.7)$ & $99.7(98.2)$ & $99.5(99)$ & $99.5(99.5)$ & 1) \\
\hline \multicolumn{8}{|l|}{ Multiplicity } \\
\hline $\mathrm{CC} 1 / 2$ & $99.8(51.2)$ & $99.8(72.4)$ & $99.6(43.6)$ & $99.9(81.6)$ & $99.6(56.8)$ & $99.8(53.7)$ & )) \\
\hline$\langle\mathrm{I} / \sigma(\mathrm{I})\rangle$ & $11(1)$ & $11.7(2.2)$ & $7.6(1)$ & $18.4(2.1)$ & $8.8(1.4)$ & $12.2(1.5)$ & ) \\
\hline $\mathrm{R}_{\text {meas }}(\%)^{\#}$ & $9.6(127)$ & $11.6(84.9)$ & $10.9(106.4)$ & $8.9(107.8)$ & $15(113.1)$ & $8.3(98.4)$ & 3) \\
\hline \multicolumn{8}{|l|}{ Refinement } \\
\hline Resolution range ( $\AA$ ) & $46.5-1.78$ & $86.5-1.94$ & $87.8-1.87$ & $87-1.82$ & $86-1.97$ & $90-1.97$ & \\
\hline $\mathrm{R}_{\text {workt }} / \mathrm{R}_{\text {free }}$ & $17.2 / 20.2$ & $16.5 / 20.4$ & $15.1 / 18.0$ & $15.5 / 18.6$ & $16.5 / 19.4$ & $17.9 / 23.0$ & 1 \\
\hline \multicolumn{8}{|l|}{ No. of non-H atoms: } \\
\hline Protein & 4,267 & 4,267 & 4,257 & 4,292 & 4,302 & 4,192 & \\
\hline FAD & 53 & 53 & 53 & 53 & 53 & 53 & \\
\hline $\mathrm{FA}^{\ddagger}$ & 20 & - & 20 & 20 & 20 & $\begin{array}{l}20(0.8) / \\
20(0.2)\end{array}$ & \\
\hline FA2 & 20 & 20 & 20 & 20 & 20 & 20 & \\
\hline $\mathrm{HDE}^{*}$ & - & 17 & - & 17 & - & & \\
\hline
\end{tabular}




\begin{tabular}{|c|c|c|c|c|c|c|}
\hline $\mathrm{CO}_{2}^{\ddagger}$ & - & 3 & - & 3 & - & - \\
\hline Bicarbonate & & & & 4 & & \\
\hline \multicolumn{7}{|l|}{ B-Factors } \\
\hline Protein & 31.7 & 30.9 & 36.3 & 35.8 & 24.2 & 35.1 \\
\hline FAD & 23.9 & 22.7 & 25.4 & 26.9 & 18.0 & 28.4 \\
\hline $\mathrm{FAl}^{ \pm}$ & 30.5 & - & 34.9 & & 30.2 & 18.7 \\
\hline FA2 & 45.8 & 44.7 & 61.2 & 52.8 & 45.3 & 45.3 \\
\hline $\mathrm{HDE}^{\ddagger}$ & - & 32 & - & 39.2 & - & - \\
\hline $\mathrm{CO}_{2}^{\ddagger}$ & - & 52 & - & 59.3 & - & - \\
\hline Ramachandran outliers (\%) & 1.1 & 0.9 & 0.4 & 0.2 & 0.2 & 0.4 \\
\hline \multicolumn{7}{|l|}{$\begin{array}{l}\text { R.m.s. deviation from ideal } \\
\text { geometry }\end{array}$} \\
\hline Bond lengths ( $\AA$ ) & 0.019 & 0.019 & 0.019 & 0.012 & 0.012 & 0.009 \\
\hline Bond angles $\left({ }^{\circ}\right)$ & 1.97 & 1.94 & 1.98 & 1.72 & 1.80 & 1.63 \\
\hline
\end{tabular}

1

2 Values in parentheses are for the highest resolution shell. ${ }^{\#} \mathrm{R}_{\text {meas }}$ corresponds to redundancy-

3 independant Rsym.. ${ }^{\star}$ Number in parentheses corresponds to the refined occupancies. *HDE

4 stands for heptadecane, the modeled product of stearate decarboxylation. 


\section{Table S2: SFX data processing and refinement statistics}

\begin{tabular}{|c|c|c|c|c|c|}
\hline Dataset & dark & light_20ps & light_900ps & light_300ns & light_2 $2 \mu \mathrm{s}$ \\
\hline PDB ID code & $6 \mathrm{ZH} 7$ & & & & \\
\hline $\begin{array}{l}\text { Pump-laser } \\
\text { excitation ( } 400 \mathrm{~nm})\end{array}$ & no & yes & yes & yes & yes \\
\hline $\begin{array}{l}\text { Nominal pump- } \\
\text { probe delay }\end{array}$ & $\mathrm{n} / \mathrm{a}$ & $20 \mathrm{ps}$ & $900 \mathrm{ps}$ & $300 \mathrm{~ns}$ & $2 \mu \mathrm{s}$ \\
\hline Space group & $P 2_{1}$ & $P 2_{1}$ & $P 2_{1}$ & $P 2_{1}$ & $P 2_{1}$ \\
\hline $\begin{array}{l}\text { Unit cell } \\
\text { parameters } \\
\mathrm{a}(\AA) \\
\mathrm{b}(\AA) \\
\mathrm{c}(\AA) \\
\beta\left({ }^{\circ}\right)\end{array}$ & $\begin{array}{l}61.4 \pm 0.1 \\
60.0 \pm 0.1 \\
182.9 \pm 0.3 \\
90.6\end{array}$ & $\begin{array}{l}61.4 \pm 0.1 \\
60.0 \pm 0.1 \\
182.9 \pm 0.3 \\
90.6\end{array}$ & $\begin{array}{l}61.4 \pm 0.1 \\
60.0 \pm 0.1 \\
182.9 \pm 0.3 \\
90.6\end{array}$ & $\begin{array}{l}61.4 \pm 0.1 \\
60.0 \pm 0.1 \\
182.9 \pm 0.3 \\
90.6\end{array}$ & $\begin{array}{l}61.4 \pm 0.1 \\
60.0 \pm 0.1 \\
182.9 \pm 0.3 \\
90.6\end{array}$ \\
\hline Collected frames & $2,579,455$ & $1,625,450$ & $1,728,093$ & $1,298,912$ & 909,645 \\
\hline Hits & 264,812 & 290,962 & 227,517 & 151,599 & 66,358 \\
\hline Indexed images & 68,421 & 88,919 & 50,214 & 44,868 & 18,600 \\
\hline Resolution ( $(\AA)$ & $\begin{array}{l}25-2.00 \\
(2.05-2.00)\end{array}$ & $\begin{array}{l}25-2.00 \\
(2.05-2.00)\end{array}$ & $\begin{array}{l}25-2.00 \\
(2.05-2.00)\end{array}$ & $\begin{array}{l}25-2.00 \\
(2.05-2.00)\end{array}$ & $\begin{array}{l}25-2.20 \\
(2.25-2.20)\end{array}$ \\
\hline Observations & $\begin{array}{l}33,069,955 \\
(1,438,474)\end{array}$ & $\begin{array}{l}42,928,992 \\
(1,869,092)\end{array}$ & $\begin{array}{l}25,083,092 \\
(1,091,803)\end{array}$ & $\begin{array}{l}20,934,706 \\
(907,081)\end{array}$ & $\begin{array}{l}8,076,033 \\
(383,644)\end{array}$ \\
\hline Unique reflections & $93,061(6,086)$ & $\begin{array}{l}93,060 \\
(6,086)\end{array}$ & $\begin{array}{l}93,064 \\
(6,086)\end{array}$ & $\begin{array}{l}93,055 \\
(6,086)\end{array}$ & $70,385(4,671)$ \\
\hline $\mathrm{R}_{\text {split }}(\%)$ & $15.1(68.5)$ & $13.4(61.3)$ & $18.0(80.0)$ & $19.4(84.5)$ & $24.9(67.8)$ \\
\hline $\mathrm{CC}^{*}$ & $0.996(0.841)$ & $0.996(0.869)$ & $0.994(0.797)$ & $0.992(0.790)$ & $0.983(0.816)$ \\
\hline $\mathrm{I} / \sigma(\mathrm{I})$ & $5.6(1.7)$ & $6.40(1.9)$ & $4.8(1.4)$ & $4.4(1.3)$ & $3.7(1.6)$ \\
\hline Completeness (\%) & $100(100)$ & $100(100)$ & $100(100)$ & $100(100)$ & $100(100)$ \\
\hline Multiplicity & $355(236)$ & $461(307)$ & $270(179)$ & $225(149)$ & $115(82)$ \\
\hline $\begin{array}{l}\mathrm{R}_{\text {iss }}{ }^{\text {(with respect }} \\
\text { to dark dataset) }\end{array}$ & n.a & 0.157 & 0.171 & 0.174 & 0.202 \\
\hline \multicolumn{6}{|l|}{ Refinement statistics } \\
\hline $\begin{array}{l}\text { Refinement } \\
\text { strategy }\end{array}$ & $\begin{array}{l}\text { Classical } \\
\text { refinement }\end{array}$ & n.a & n.a & n.a & n.a \\
\hline Resolution ( $(\AA)$ & $\begin{array}{l}25-2.00 \\
(2.05-2.00)\end{array}$ & & & & \\
\hline$R_{\text {free }}$ & 0.235 & & & & \\
\hline $\mathrm{R}_{\text {work }}$ & 0.196 & & & & \\
\hline $\begin{array}{l}\text { Number of protein } \\
\text { atoms }\end{array}$ & 8417 & & & & \\
\hline $\begin{array}{l}\text { Number of ligand } \\
\text { atoms }\end{array}$ & 166 & & & & \\
\hline
\end{tabular}




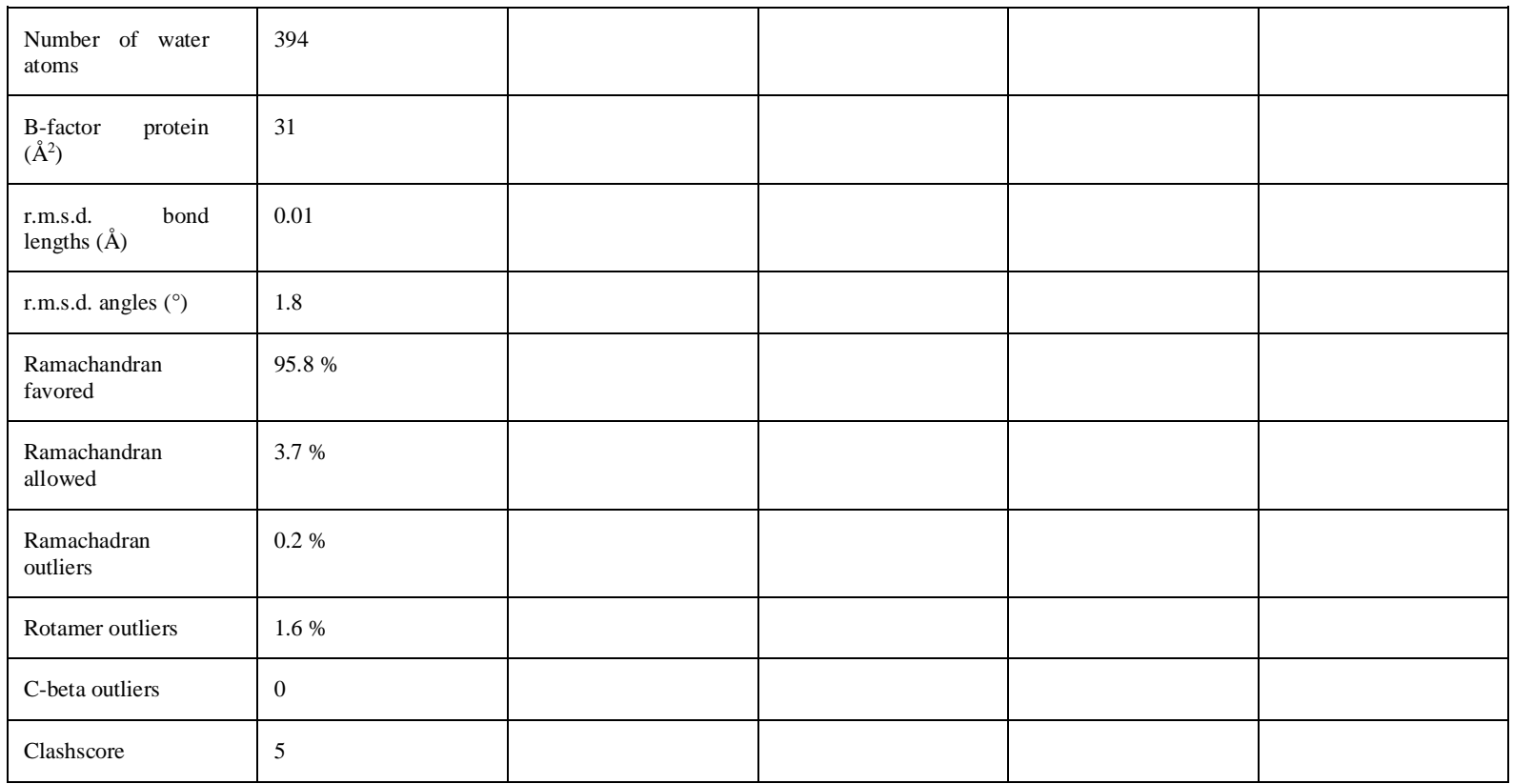

1 Values in brackets are for the highest resolution shell

$$
\#: R_{\text {split }}=\frac{1}{\sqrt{2}} \times \frac{\sum_{h k l}\left|I_{h k l}^{\text {even }}-I_{h k l}^{\text {odd }}\right|}{0.5 \times \sum_{h k l}\left|I_{h k l}^{\text {even }}+I_{h k l}^{\text {odd }}\right|}
$$

$3 \quad \mathrm{CC}^{*}:$ see $(121)$

$4 \quad \mathrm{R}_{\text {iso }}^{\$}$ was calculated using Phenix up to 2.2 A resolution 
Table S3: Primers list

\begin{tabular}{|c|c|}
\hline \multirow{2}{*}{$\begin{array}{c}\text { FAP (76- } \\
654)\end{array}$} & 5'-aacctgtacttccaatcaagtccggttgca-3' \\
\hline & 5'-tatccacetttactgttatcatgctgcaacggttgccggtg-3, \\
\hline \multirow{2}{*}{ R451A } & 5'-catacceggaacaaaagctacctgcagatccggc-3' \\
\hline & 5'-gccggatctgcaggtagettttgttcegggtatg-3' \\
\hline \multirow{2}{*}{ R451K } & 5'-gtgccatacceggaacaaactttacetgcagatccggcagt-3, \\
\hline & 5'-actgceggatctgcaggtaaagtttgttcegggtatggcac-3, \\
\hline \multirow{2}{*}{ Y466F } & 5'-ttttgcaaaacgaacaaaggtgctaacaccatccgg-3, \\
\hline & 5'-ccggatggtgttagcacctttgttcgttttgcaaaa-3', \\
\hline
\end{tabular}

2

3

4

5

6

7

8

9

10

11 
1 Table S4: list of sequences of FAPs and other GMC oxidoreductases used for protein

2 alignment and sequence logo.

3 Accession numbers are indicated between parentheses.

\begin{tabular}{|c|c|}
\hline Putative FAPs & $\begin{array}{c}\text { Glucose methanol choline } \\
\text { oxidoreductases } \\
\text { (other than FAP) }\end{array}$ \\
\hline Aureococcus anophagefferens (F0YAB1) & Aspergillus niger (J05242) \\
\hline Chlamydomonas reinhardtii (XP_001703004) & Brevibacterium sterolicum (P22637) \\
\hline Chlorella variabilis NC64A (KY511411) & Caenorhabditis elegans (Q18429) \\
\hline Chondrus crispus (XP_005714951) & Candida boidinii (Q00922) \\
\hline Chrysochromulina (KOO25289) & Caulobacter crescentus(AAK22929) \\
\hline $\begin{array}{c}\text { Coccomyxa subellipsoidea } \mathrm{C}-169 \\
\text { (XP_005642926) }\end{array}$ & Drosophila melanogaster(NM_058155) \\
\hline Cyanidioschyzon merolae (XP_005537774) & Escherichia coli (NP414845) \\
\hline Dunaliella salina (Dusal.0308s00018.1) & Gluconobacter oxydans (BAA13145) \\
\hline Ectocarpus siliculosus (CBJ25560) & Halococcus sediminicola (WP_049996878) \\
\hline $\begin{array}{c}\text { Fragilariopsis cylindrus CCMP1102 } \\
\text { (A0A1E7FBP9) }\end{array}$ & Phanerochaete chrysosporium (U46081) \\
\hline Galdieria sulphuraria (XP_005706847) & Phytophthora parasitica (W2PSU1) \\
\hline Nannochloropsis gaditana (EWM27492) & Pleurotus pulmonarius (AAF31169) \\
\hline $\begin{array}{c}\text { Phaeodactylum tricornutum CCAP 1055/1 } \\
\text { (B7FSU6) }\end{array}$ & Prunus dulcis (O24243) \\
\hline \multirow[t]{3}{*}{ Volvox carterif. nagariensis (XP_002948047) } & Pseudomonas fluorescens (ZP_00084192) \\
\hline & Pseudomonas oleovorans (Q00593) \\
\hline & Sphingopyxis terrae (BAB61732) \\
\hline
\end{tabular}

4 
1 Table S5: R-/U-B3LYP energies and energy gradient of the optimized geometries

2 modelling alkane production by the FAP enzyme.

3 The active site model is presented in fig. S16. CS and DR(CT) stands for closed-shell and

4 diradical (charge-transfer).

\begin{tabular}{|c|c|c|c|c|}
\hline $\begin{array}{l}\text { Geometry } \\
\text { and } \\
\text { electronic } \\
\text { configuratio } \\
\text { n }\end{array}$ & $\begin{array}{l}\text { dark state/ } \\
\text { intermediate/ } \\
\text { product }\end{array}$ & $\begin{array}{l}\text { Energy, } \quad \text { au } \\
\text { (energy relative } \\
\text { to }(1), \mathrm{kcal} / \mathrm{mol} \text { ) }\end{array}$ & $\begin{array}{l}\text { Max energy grad, } \\
\text { au }\end{array}$ & $\begin{array}{l}\text { RMS } \\
\text { grad, au }\end{array}$ \\
\hline (1) $\mathrm{CS}$ & dark state & $\begin{array}{l}-6524.996965 \\
(0.0)\end{array}$ & 0.0002 & 0.0001 \\
\hline (2) $\mathrm{CS}$ & $\begin{array}{l}\text { Unstable structure with } \\
\text { rearranged Wat } 1\end{array}$ & $\begin{array}{l}-6524.962562 \\
(21.6)\end{array}$ & 0.006 & 0.0007 \\
\hline (3) $\mathrm{DR}(\mathrm{CT})$ & $\begin{array}{l}\text { Intermediate } \mathrm{FAD}^{\bullet-}- \\
\text { and alkyl radicals, } \mathrm{CO}_{2}\end{array}$ & $\begin{array}{l}-6524.914696 \\
(51.6)\end{array}$ & 0.0003 & 0.0001 \\
\hline (4) $\mathrm{DR}(\mathrm{CT})$ & $\begin{array}{l}\text { Intermediate } \mathrm{FAD}^{\bullet-}- \\
\text { and } \mathrm{C} 432 \text { radicals, } \mathrm{CO}_{2}\end{array}$ & $\begin{array}{l}-6524.938438 \\
(36.7)\end{array}$ & 0.0003 & 0.0001 \\
\hline (5) $\mathrm{CS}$ & $\begin{array}{l}\text { Intermediate; alkane, } \\
\mathrm{C} 432 \text { anion, } \mathrm{CO}_{2}\end{array}$ & $\begin{array}{l}-6524.943038 \\
(33.8)\end{array}$ & 0.0003 & 0.0001 \\
\hline (5a) $\mathrm{CS}$ & $\begin{array}{l}\text { Intermediate; alkane } \\
\text { neutral R541, } \mathrm{CO}_{2}\end{array}$ & $\begin{array}{l}-6524.942524 \\
(34.2)\end{array}$ & 0.0005 & 0.0001 \\
\hline (5b) $\mathrm{CS}$ & $\begin{array}{l}\text { Intermediate; alkane } \\
\text { deprot } \mathrm{R} 451, \mathrm{CO}_{2}\end{array}$ & $\begin{array}{l}-6524.953052 \\
(27.6)\end{array}$ & 0.0003 & 0.0001 \\
\hline (6) $\mathrm{CS}$ & $\begin{array}{l}\text { Product state; } \\
\text { bicarbonate derived } \\
\text { from Wat } 1\end{array}$ & $\begin{array}{l}-6524.986142 \\
(6.8)\end{array}$ & 0.0004 & 0.0001 \\
\hline (6a) CS & $\begin{array}{l}\text { Product state; } \\
\text { bicarbonate derived } \\
\text { from Wat } 2\end{array}$ & $\begin{array}{l}-6524.968644 \\
(17.1)\end{array}$ & 0.0011 & 0.0001 \\
\hline
\end{tabular}

5 
1 Table S6: Excitation energy calculations for geometries (1) and (2).

2 Excitation energy, eV/ transition dipole moment, au/ state assignment are indicated for each

3 state. The details of the XMCQDPT2-CASSCF calculations are given for each geometry.

\begin{tabular}{|c|c|c|c|}
\hline State & (1) $\mathrm{sa} 8(14,8)$ & (1)-wo-Wat2 sa10 $(18,10)$ & (2) $\operatorname{sa} 8(14,8)$ \\
\hline 1 & $2.36 / 0.515 / \mathrm{Fl} \mathrm{n} \pi^{* a)}$ & $2.36 / 0.578 / \mathrm{Fl} n \pi^{*}$ & $1.87 / 0.438 / \mathrm{CT} F A$ \\
\hline 2 & $2.70 / 2.302 / \mathrm{Fl} \pi \pi^{* \mathrm{~b})}$ & 2.71/ 2.294/ Fl $\pi \pi^{*}$ & $2.33 / 0.316 / \mathrm{Fl} \mathrm{n} \pi^{*}$ \\
\hline 3 & $3.41 / 0.373 /$ CT A171-T172 ${ }^{\mathrm{c}}$ & $2.81 / 0.279 / \mathrm{CT} \mathrm{FA}^{\mathrm{c})}$ & $2.54 / 2.244 / \mathrm{Fl} \pi \pi^{*}$ \\
\hline 4 & $3.54 / 1.889 / \mathrm{Fl} \pi \pi^{* \mathrm{~b})}$ & 3.48/ 0.908/ CT A171-T172 & 3.01/ 0.472/ CT FA \\
\hline 5 & $4.01 / 0.004 / \mathrm{CT} Y 466^{\mathrm{c})}$ & $3.53 / 1.149 /$ CT FA $^{c)}$ & $3.25 / 2.136 /$ Fl $\pi \pi^{*}$ \\
\hline 6 & 4.64/ 0.143/ CT C432 c) & $3.60 / 1.212 / \mathrm{Fl} \pi \pi^{*}$ & $3.37 / 0.010 / \mathrm{CT}$ Y466 \\
\hline 7 & $5.12 / 0.004 / \mathrm{CT} \mathrm{Y} 466^{\mathrm{c})}$ & 4.14/ 0.001/ CT Y466 & $4.46 / 0.002 / \mathrm{CT}$ Y466 \\
\hline 8 & & 4.83/ 0.014/ CT C432 & \\
\hline 9 & & $5.25 / 0.007 /$ CT Y466 & \\
\hline
\end{tabular}

4 a) $n \pi *$ state of flavin involving the lone pair of N5 with a low osc. strength;

5 b) $\pi \pi^{*}$ state flavin with a high osc. strength corresponding to an absorption band;

6 c) $\pi \pi^{*}$ charge-transfer (CT) state from the protein (involved residues indicated) and fatty acid

7 (FA) to flavin with a low oscillator strength.

8

9

10 
1 Table S7: Electronic couplings for states involved in fET and bET.

2 For a pair of states $\mathrm{i}$ and $\mathrm{j}$, we indicate the energy gap $\left(\Delta \mathrm{E}_{\mathrm{ij}}\right)$, transition dipole moment $\left(\mathrm{tdm}_{\mathrm{ij}}\right)$, 3 and dipole moment change $\left(\Delta \mathrm{d}_{\mathrm{ij}}\right)$. These quantities determine the electronic coupling $\left(\mathrm{V}_{\mathrm{ij}}\right)$ The

4 geometry for which excited states were computed, details of the XMCQDPT2-CASSCF

5 calculations and shortest distance between the donor and acceptor (DA distance) are given. fET

6 and bET stand for forward and back electron transfer, respectively.

7

8

9

10

11

12

13

14

15

16

17

18

19 
1

2

3

4

5

6

7

8

9

10

11

12

13

14

15

16

17

\begin{tabular}{|c|l|l|c|c}
\hline $\begin{array}{c}\text { Transition between states } \\
\mathrm{i} \text { and } \mathrm{j}\end{array}$ & $\Delta \mathrm{E}_{\mathrm{ij}}, \mathrm{au}$ & $\mathrm{tdm}_{\mathrm{ij}}, \mathrm{au}$ & $\begin{array}{c}\Delta \mathrm{d}_{\mathrm{ij}}, \\
\mathrm{au}\end{array}$ & $\mathrm{V}_{\mathrm{ij}}, \mathrm{meV}$ \\
\hline
\end{tabular}

Coupling between flavin and protein (A171-T172)

\begin{tabular}{|c|c|c|c|c|}
\hline \multicolumn{5}{|c|}{ Geometry (1); sa, distance $2.00 \AA$} \\
\hline states 3 and 4 & 0.020848 & 0.616 & 8.5 & 40 \\
\hline \multicolumn{5}{|c|}{ C432S geometry $\left(1^{\mathrm{C} / \mathrm{S}}\right)$-wo-W3; sa8 $(14,8)$; DA distance $2.07 \AA$} \\
\hline states 3 and 5 & 0.027289 & 0.264 & 8.7 & 22 \\
\hline
\end{tabular}

Geometry (1)-wo-Wat2; sa10(18,10); DA distance $3.06 \AA$

\begin{tabular}{|c|c|c|c|c|}
\hline states 2 and 3 & 0.003863 & 1.385 & 8.3 & 17 \\
\hline \multicolumn{6}{|c|}{ Geometry (2); sa8(14,8); DA distance 2.81 } \\
\hline states 1 and 3 & 0.024880 & 0.599 & 8.5 & 47 \\
\hline
\end{tabular}

C432S geometry (1 $\left.{ }^{\mathrm{C} / \mathrm{S}}\right)$-wo-Wat2; sa8(14,8); DA distance $4.77 \AA$

\begin{tabular}{|c|l|l|l|c|}
\hline states 2 and 3 & 0.009329 & 0.599 & 11.4 & 13 \\
\hline \multicolumn{6}{|c|}{ C432S geometry $\left(2^{\mathrm{C} / \mathrm{S}}\right)$; sa7(12,12); DA distance 4.84 $⿱$} \\
\hline states 3and 4 & 0.004058 & 0.799 & 11.4 & 8 \\
\hline
\end{tabular}

alkyl and Y466 coupling

Geometry (3), sa5(8,5); DA distance $3.69 \AA$

\begin{tabular}{|c|l|l|l|c|}
\hline states 1 and 5 & 0.166660 & 0.223 & 7.1 & 142 \\
\hline \multicolumn{5}{|c|}{ Couplings for bET } \\
\hline Geometry (3), sa5(8,5); distance 6.57 \\
\hline states 0 and 3 & 0.115436 & 0.029 & 14.8 & 6 \\
\hline \multicolumn{6}{|c|}{ Geometry (4); sa7(12,7); distance 6.93 } \\
\hline states 0 and 2 & 0.030510 & 0.053408 & 15.7 & 3 \\
\hline states 1and 2 & 0.023301 & 0.088748 & 15.7 & 4 \\
\hline
\end{tabular}


1 Table S8: Excitation energy calculations for suggested oxidized flavin red-shifted

2 intermediates (5), (5b), (6) and (6a).

3 Excitation energy, eV/ transition dipole moment, au/ state assignment are indicated for each

4 state. The XMCQDPT2-CASSCF sa7(12,7) calculations for all geometries except (5).

\begin{tabular}{|c|c|c|c|c|c|}
\hline $\begin{array}{l}\text { Stat } \\
\text { es }\end{array}$ & (1) & $(5) ; \mathrm{sa} 8(14,8)$ & (5b) & (6) & (6a) \\
\hline 1 & $2.31 / 0.590 / \mathrm{Fl} \mathrm{n} \pi^{*}$ & $\begin{array}{l}0.91 / 0.054 / \mathrm{CT} \\
\mathrm{C} 432\end{array}$ & $2.23 / 0.436 / \mathrm{Fl} \mathrm{n} \pi *$ & $2.30 / 0.516 / \mathrm{Fl} n \pi^{*}$ & $2.29 / 0.617 / \mathrm{Fl} \mathrm{n} \pi *$ \\
\hline 2 & $2.70 / 2.253 / \mathrm{Fl} \pi \pi^{*}$ & $\begin{array}{l}1.21 / 0.107 / \mathrm{CT} \\
\mathrm{C} 432\end{array}$ & $2.56 / 2.275 / \mathrm{Fl} \pi \pi^{*}$ & $2.64 / 2.260 / \mathrm{Fl} \pi \pi^{*}$ & $2.65 / 2.250 / \mathrm{Fl} \pi \pi^{*}$ \\
\hline 3 & $3.57 / 1.959 / \mathrm{Fl} \pi \pi^{*}$ & $2.62 / 2.377 / \mathrm{F} 1 \pi \pi^{*}$ & 2.63/ 0.012/ CT R541 & $3.47 / 2.011 / \mathrm{Fl} \pi \pi^{*}$ & $3.53 / 1.980 / \mathrm{Fl} \pi \pi^{*}$ \\
\hline 4 & 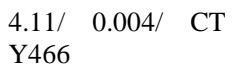 & $\begin{array}{l}3.08 / 0.001 / \mathrm{CT} \\
\text { Y466 }\end{array}$ & $3.33 / 0.000 /$ CT Y466 & $\begin{array}{lll}3.90 / & 0.001 / & \mathrm{CT} \\
\mathrm{Y} 466 & & \end{array}$ & $\begin{array}{lll}4.07 / & 0.001 / & \mathrm{CT} \\
\mathrm{Y} 466 & \end{array}$ \\
\hline 5 & $\begin{array}{lll}4.64 / & 0.143 / & \mathrm{CT} \\
\mathrm{C} 432 & \end{array}$ & $\begin{array}{l}3.24 / 0.003 / \mathrm{CT} \\
\mathrm{C} 432\end{array}$ & $3.47 / 2.003 / \mathrm{Fl} \pi \pi^{*}$ & $\begin{array}{lll}4.64 / & 0.008 / & \mathrm{CT} \\
\mathrm{C} 432 & & \end{array}$ & $\begin{array}{lll}4.67 / & 0.006 / & \mathrm{CT} \\
\mathrm{C} 432 & & \end{array}$ \\
\hline 6 & $\begin{array}{lll}5.12 / & 0.004 / & \mathrm{CT} \\
\mathrm{Y} 466 & & \end{array}$ & $3.66 / 1.877 / \mathrm{Fl} \pi \pi^{*}$ & $3.82 / 0.004 / \mathrm{CT}$ C432 & $\begin{array}{lll}5.01 / & 0.002 / & \mathrm{CT} \\
\mathrm{Y} 466 & & \end{array}$ & $\begin{array}{lll}5.19 / & 0.008 / & \mathrm{CT} \\
\mathrm{Y} 466 & & \end{array}$ \\
\hline 7 & & $\begin{array}{l}4.26 / 0.016 / \mathrm{CT} \\
\text { C432 }\end{array}$ & & & \\
\hline
\end{tabular}

5

6 
1 Table S9: Effect of butterfly bending on flavin redox and excitation energies.

2 At the indicated geometries, energies of the flavin oxidized and radical-anion states were

3 computed. The oxidized flavin was also computed in the excited state (energies given in

4 brackets). In addition to isoalloxazine, a FAP active site model (AS) was considered (the model

5 is presented in fig. S16). To facilitate comparison, relative energies were computed. To discuss

6 the effect of flavin bending (see fig. S21), we compare electron affinities $\left(\mathrm{VEA}^{\mathrm{a}}{ }^{\text {) }}\right.$ and $\mathrm{AEA}{ }^{\mathrm{b}}$ )

7 and excitation energies $\left(\mathrm{VE}^{\mathrm{c})}\right)$. The negative EAs indicate that oxidized flavin is higher in energy

8 than radical anion.

\begin{tabular}{|c|c|c|c|c|}
\hline \multirow[t]{2}{*}{ Geometry } & \multicolumn{2}{|l|}{ Energy, au } & \multicolumn{2}{|c|}{ Relative energy, eV } \\
\hline & oxidized & rad. anion & oxidized & rad. anion \\
\hline Planar oxidized & $\begin{array}{l}-986.177790 \\
(-986.066712)\end{array}$ & -986.219889 & $\begin{array}{l}0.0 \\
\text { VE: } 3.02\end{array}$ & VEA: -1.15 \\
\hline Planar rad-an & -986.170156 & -986.235240 & 0.21 & $\mathrm{AEA}^{\mathrm{e})}:-1.57$ \\
\hline Oxidized, $20 \mathrm{deg}$ d) & $\begin{array}{l}-986.170943 \\
(-986.064903)\end{array}$ & -986.220929 & $\begin{array}{l}0.0 \\
\text { VE: } 2.89\end{array}$ & VEA: -1.36 \\
\hline Rad-an, 20 deg & -986.162278 & -986.230043 & 0.24 & AEA: -1.61 \\
\hline Oxidized, $30 \mathrm{deg}$ & $\begin{array}{l}-986.159248 \\
(-986.056440)\end{array}$ & -986.207741 & $\begin{array}{l}0.0 \\
\text { VE: } 2.80\end{array}$ & VEA: -1.32 \\
\hline Rad-an, 30 deg & -986.151201 & -986.222770 & 0.22 & AEA: -1.73 \\
\hline $\begin{array}{l}\text { Oxidized in AS, } 15 \text { deg } \\
\text { e) }\end{array}$ & $\begin{array}{l}-4378.641248 \\
(-4378.541981)\end{array}$ & -4378.788512 & $\begin{array}{l}0.0 \\
\text { VE: } 2.70\end{array}$ & VEA: -4.00 \\
\hline $\begin{array}{l}\text { Radical anion in AS, } \\
20 \text { deg f }\end{array}$ & -4378.645995 & -4378.841320 & -0.13 & AEA: -5.44 \\
\hline
\end{tabular}

9 a) Vertical electron affinity (VEA), the energy change due to adding one electron to the 10 oxidized flavin at the oxidized-flavin optimized geometry; 
1 b) adiabatic electron affinity (AEA), the energy change due to adding one electron to the 2 oxidized flavin taking into account geometry relaxation;

3 c) vertical excitation (VE), the energy of the first flavin $\pi \pi^{*}$ computed with TD-B3LYP at 4 the oxidized-flavin optimized geometry;

5 d) the constraint butterfly bending angle;

6 e) the butterfly bending angle after geometry optimization of the dark state (1);

7 f) the butterfly bending angle after geometry optimization of the radical intermediate (3). 
1 Table S10: Excitation energy calculations for C432S model: effect of Wat3.

2 Excitation energy, eV/ transition dipole moment, au/ state assignment are indicated for each 3 state. Details of the XMCQDPT2-CASSCF calculations are given for each model.

\begin{tabular}{|c|c|c|c|c|}
\hline $\begin{array}{l}\text { Exc. } \\
\text { state }\end{array}$ & $\left(1^{\mathrm{C} / \mathrm{S}}\right) \mathrm{sa} 6(10,6)$ & $\begin{array}{l}\left(1^{\mathrm{C} / \mathrm{S}}\right)-\text { wo-Wat } 2 \\
\text { sa8 }(14,8)\end{array}$ & $\begin{array}{l}\left(1^{\mathrm{C} / \mathrm{S}}\right) \text {-wo-Wat3 } \\
\text { sa } 8(14,8)\end{array}$ & $\left(2^{\mathrm{C} / \mathrm{S}}\right) \mathrm{sa} 7(12,7)$ \\
\hline 1 & $2.28 / 0.528 / \mathrm{Fl} \mathrm{n} \pi *$ & $2.24 / 0.516 / \mathrm{Fln} n \pi^{*}$ & $2.28 / 0.530 / \mathrm{Fl} \mathrm{n} \pi^{*}$ & $2.27 / 0.644 / \mathrm{Fl} \mathrm{n} \pi^{*}$ \\
\hline 2 & $2.71 / 2.234 / \mathrm{Fl} \pi \pi^{*}$ & $2.73 / 2.360 / \mathrm{Fl} \pi \pi^{*}$ & $2.71 / 2.347 / \mathrm{Fl} \pi \pi^{*}$ & $2.55 / 0.212 / \mathrm{CT} \mathrm{FA}^{\mathrm{d})}$ \\
\hline 3 & 3.71/1.915/Fl $\pi \pi^{*}$ & $\begin{array}{l}2.99 / 0.124 / \mathrm{CT} \mathrm{FA}^{\text {a) }} \\
\text {,b) }\end{array}$ & $2.98 / 0.181 / \mathrm{CT} \mathrm{FA}^{\mathrm{b})}$ & $2.66 / 2.303 / \mathrm{Fl} \pi \pi^{*}$ \\
\hline 4 & $\begin{array}{l}4.02 / 0.001 / \mathrm{CT} \\
\mathrm{Y} 466\end{array}$ & $3.74 / 1.853 / \mathrm{Fl} \pi \pi^{*}$ & $\begin{array}{l}3.45 / 0.201 / \mathrm{CT} \\
\mathrm{T}_{172^{\mathrm{c})}}\end{array}$ & $\begin{array}{l}3.55 / 0.014 / \mathrm{CT} \\
\text { Y } 466\end{array}$ \\
\hline 5 & $\begin{array}{l}5.16 / 0.009 / \mathrm{CT} \\
\text { Y466 }\end{array}$ & $3.89 / 0.207 / \mathrm{CT} F A$ & $3.72 / 1.858 / \mathrm{Fl} \pi \pi^{*}$ & 3.56/0.197/CT FA \\
\hline 6 & & $\begin{array}{l}4.18 / 0.001 / \mathrm{CT} \\
\text { Y466 }\end{array}$ & 4.05/0.001/CT Y466 & $3.66 / 1.937 / \mathrm{Fl} \pi \pi^{*}$ \\
\hline 7 & & $\begin{array}{l}5.32 / 0.003 \quad \text { CT } \\
\text { Y466 }\end{array}$ & 5.16/0.004/CT Y466 & \\
\hline
\end{tabular}

a) this energy in comparison to a similar energy of the dark state (1)-wo-Wat2 $(2.81 \mathrm{eVm}$ Table S6) indicates an increase of the CT-fatty acid (FA) energy by interactions of the substrate with Wat3;

7 b) removing of Wat 3 and Wat 2 both reduce the energy of electron abstraction by flavin 8 from the fatty acid carboxylate to the same energy level, about $3 \mathrm{eV}$ above the ground-state $9 \quad$ energy;

10 c) removing Wat3 reduces the energy of electron transfer from A171-T172 donor to the 11 flavin. This energy is comparable to the respective energy in the WT active site model (1) 

fatty acid energy is lower than the flavin $\pi \pi^{*}$ energy.

5 Table S11: Excitation energy calculations for diradical intermediates (3) and (4).

6 Excitation energy, eV/ transition dipole moment, au/ state assignment are indicated for each

7 state. The details of the XMCQDPT2-CASSCF calculations are given for each geometry.

\begin{tabular}{|l|l|l|}
\hline Ext. state & $(3) \mathrm{sa}(8,5)$ & $(4) \mathrm{sa} 7(12,7)$ \\
\hline 1 & $2.44 / 1.886 / \mathrm{Fl} \mathrm{rad}-\mathrm{an}$ & $0.20 / 0.025 / \mathrm{C} 432 \mathrm{rad}$ \\
\hline 2 & $2.97 / 2.220 / \mathrm{Fl} \mathrm{rad}-\mathrm{an}$ & $0.83 / 0.053 / \mathrm{bET}$ \\
\hline 3 & $3.14 / 0.029 / \mathrm{bET}$ & $2.67 / 2.184 / \mathrm{Fl} \mathrm{rad}-\mathrm{an}$ \\
\hline 4 & $4.64 / 0.131 / \mathrm{CT} \mathrm{Y} 466$ & $2.87 / 0.131 / \mathrm{Fl} \& \mathrm{C} 432$ \\
\hline 5 & & $3.21 / 1.945 / \mathrm{Fl} \mathrm{rad}-\mathrm{an}$ \\
\hline 6 & & $3.41 / 0.004 / \mathrm{Fl} \& \mathrm{C} 432$ \\
\hline
\end{tabular}

8 